


\section{Vascularization of Diabetes-reversing Implants:}

Development of Sustained Release Formulations of Proangiogenic Growth Factors

Karina Christiane Scheiner 
Vascularization of Diabetes-reversing Implants: Development of Sustained Release Formulations of Proangiogenic Growth Factors

Karina Christiane Scheiner

PhD Thesis with summary in English, German and Dutch

(C)2020 Karina Christiane Scheiner

All rights reserved. No part of this thesis may be reproduced or transmitted in any form by any means without prior permission in writing of the copyright owner.

This thesis was (partly) accomplished with financial support from the European Union's Horizon 2020 research and innovation program under grant agreement number 645991 (DRIVE).

ISBN: 978-94-6375-804-8

Cover design and lay-out: Iliana Boshoven-Gkini, www.AgileColor.com

Printing: Ridderprint, www.ridderprint.nL

The printing of this thesis was financially supported by Utrecht Institute for Pharmaceutical Sciences (UIPS), InnoCore BV, Micropore Technologies, PeproTech EC Ltd, INNOVA S.r.I. 


\title{
Vascularization of Diabetes-reversing Implants: Development of Sustained Release Formulations of Proangiogenic Growth Factors
}

\author{
Vascularisatie van een Kunstmatige Alvleesklier: \\ Ontwikkeling van Formuleringen met Vertraagde Afgifte \\ van Proangiogene Groeifactoren \\ (met een samenvatting in het Nederlands)
}

Vaskularisation einer künstlichen Bauchspeicheldrüse:

Entwicklung von Formulierungen mit verzögerter Freisetzung von Proangiogenen Wachstumsfaktoren

(mit einer Zusammenfassung in deutscher Sprache)

\section{Proefschrift}

ter verkrijging van de graad van doctor aan de

Universiteit Utrecht

op gezag van de

rector magnificus, prof.dr. H.R.B.M. Kummeling, ingevolge het besluit van het college voor promoties

in het openbaar te verdedigen op

maandag 9 maart 2020 des middags te 2.30 uur

door

\section{Karina Christiane Scheiner}

geboren op 28 april 1988

te Burlington, Canada 


\section{Promotor:}

Prof. dr. ir. W.E. Hennink

\section{Copromotor:}

Dr. R.J. Kok 
"If we knew what we were doing, it wouldn't be called research, would it?" Albert Einstein 



\section{Table of Contents}

$\begin{array}{lll}\text { Chapter } 1 \text { General Introduction } & 9\end{array}$

Chapter 2 Sustained Release of Vascular Endothelial Growth Factor from

Poly( $\varepsilon$-caprolactone-PEG-e-caprolactone)-b-Poly(L-lactide)

Multiblock Copolymer Microspheres

Chapter 3 Vascular Endothelial Growth Factor-releasing Microspheres based on Poly( $\varepsilon$-caprolactone-PEG- $\varepsilon$-caprolactone)-b-Poly(Llactide) Multiblock Copolymers incorporated in a 3D-printed Poly(dimethylsiloxane) Cell Macroencapsulation Device

Chapter 4 Self-encapsulation of Proangiogenic Growth Factors in PLGA Microspheres

Chapter 5 Sustained Release of Vascular Endothelial Growth Factor from Chondroitin Sulfate-based Hydrogels

Chapter 6 Summary and Perspectives 



\section{Chapter 1}

General Introduction 


\section{Diabetes mellitus type 1}

Diabetes mellitus is a severe, chronic endocrine disease caused by insulin deficiency (type 1) or insulin insensitivity (type 2). While type 1 diabetes is typically diagnosed in the early years of childhood, type 2 diabetes often develops in adult years as a consequence of poor dietary practices and obesity. In 2014, more than 400 million adults worldwide were affected by diabetes, with the vast majority by type 2 diabetes.' The global incidence rate of type 1 diabetes varies from less than 0.5 cases per 100.000 children under 15 years in Asia and Latin America to up to $60 / 100.000$ Scandinavian countries, dramatically increasing each year., ${ }^{2,3}$

Pathologically, type 1 diabetes is caused by irreversible autoimmune destruction of $\beta$-cells. ${ }^{4}$ As a crucial component of pancreatic islets, $\beta$-cells secrete insulin, a peptide hormone which regulates the uptake of glucose into cells. ${ }^{5}$ The lack of endogenous insulin production and secretion, without appropriate treatment, is potentially life-threatening. 'The current therapy standard aims at mimicking and substituting normal physiological insulin levels with injections of recombinant long-acting and/or short-acting insulin analogues to achieve glycemic control. The dose of administered insulin is adjusted to the blood glucose value measured prior to injection via insulin pens or pumps. Although insulin therapy offers patients independency, control of their condition and an increased life expectancy, this therapy requires a high level of comprehension, discipline and compliance and thus has a great impact on a patient's everyday-life. ${ }^{6,7}$ Consequently, insulin therapy poses a great challenge for juvenile patients ${ }^{8}$ and patients affected by brittle diabetes, a condition of poor glycemic stability, which can lead to severe, life-threatening hypoglycemia., 10 


\section{Pancreatic islet transplantation}

The transplantation of healthy pancreatic islets is an emerging treatment approach to restore endogenous insulin production in type 1 diabetes patients. ${ }^{11}$ Through the initially developed Edmonton protocol, purified donor pancreatic islets are injected via the patient's portal vein, engrafting the islets in the highly vascularized liver tissue (figure 1).

Since the first reports of this protocol in 2000,12 hundreds of patients have achieved yearslong insulin-independence after pancreatic islet transplantation. ${ }^{13}$ However, pancreatic islet transplantation via the Edmonton protocol still has many hurdles to overcome before becoming a clinical reality for diabetes patients. Post transplantation, a continuous intake of strong immunosuppressive drugs is necessary to prevent rejection of the islets. Further limitations include poor islet survival and the lack of donor pancreases, as on average three donor pancreases are needed per recipient.12,14

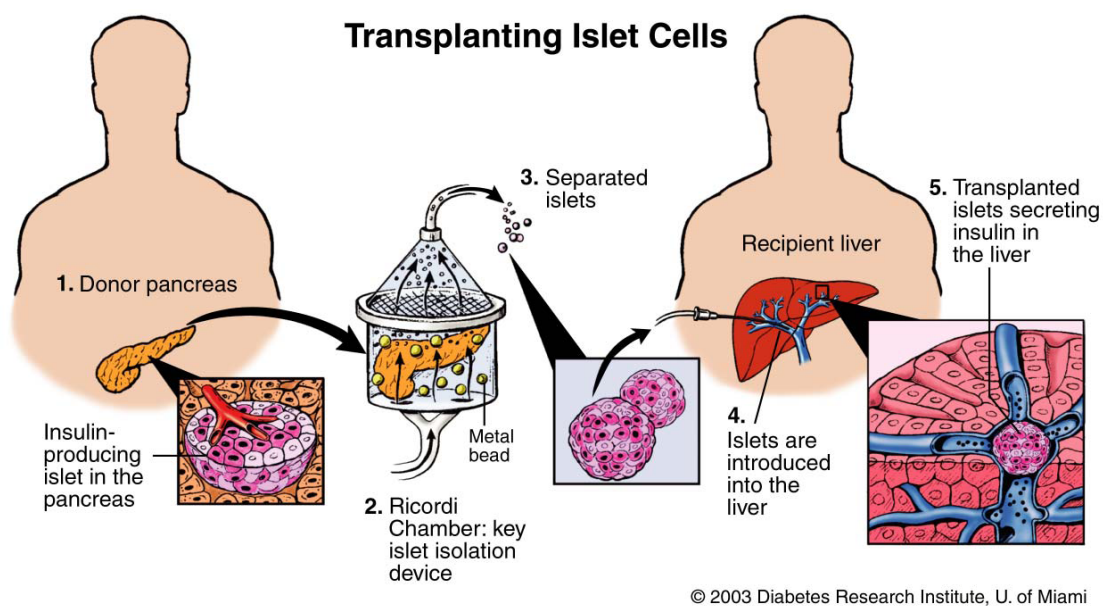

Artist: Robert Margulies

FIGURE 1: Pancreatic islet transplantation based on the Edmonton protocol. The pancreatic islets are isolated and purified, before injection into the portal vein of the recipient liver..$^{15}$ C Diabetes Research Institute, University of Miami, USA. Reproduced with permission.

Recent developments havefocused on (macro-)encapsulating pancreatic islets into biomaterial devices to increase transplantation efficiency. ${ }^{16,17}$ Typically, these devices are encompassed by a semi-permeable membrane. This forms an immune barrier, preventing immune-related rejection of the islets while allowing the transport of insulin and waste products from encapsulated islets and oxygen and nutrients to the islets ${ }^{17}, 18$. Such a combination of cells with (biologically active) biomaterials in a complex devices is classified as advanced therapy medicinal products (ATMP). 19, 20 Several developed ATMPs for diabetes treatment are currently in phase I and II clinical trials (figure 2). Commonly, these pouch-like devices are implanted subcutaneously via minimally-invasive surgery. ${ }^{19}$ 
a
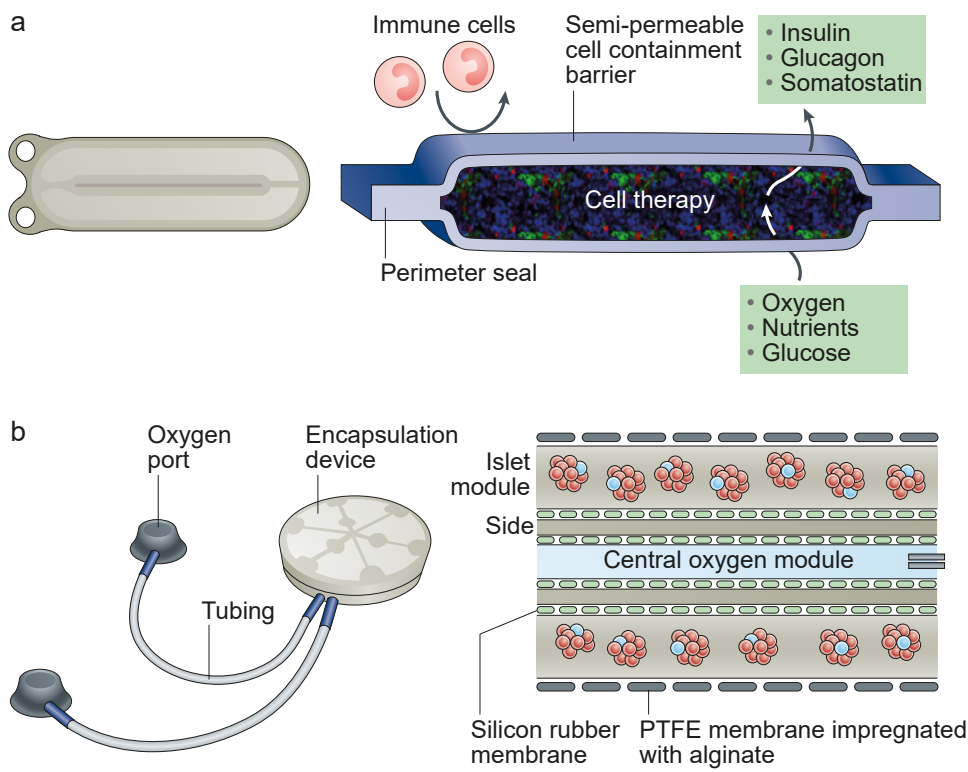

FIGURE 2: Pancreatic islet encapsulation devices ViaCyte and Beta-O ${ }_{2}$. The ViaCyte device (a) consisting of a semipermeable cell container, which shields the encapsulated cells from host immune cells but still allows the transport of insulin, oxygen and nutrients. The Beta- $\mathrm{O}_{2}$ device (b) consists of a central module in which pancreatic islet are embedded in a semi-permeable membrane. Two ports allow for oxygen supply and islet replenishment. PTFE: polytretrafluoroethylene. Adapted from Desai et al.,17 reproduced with permission.

\section{Vascularization strategies}

Although remarkable progress towards long-term insulin-independence has been made with islet encapsulation devices, the lack of vascularization of the device is a major challenge for successful transplantation. ${ }^{16,17,21,22}$ Insufficient supply of nutrients and oxygen to the islets and hypoxia decreases islet survival. ${ }^{21}$ This is even more striking when considering that the process of blood vessel formation (angiogenesis) toward the device takes around four weeks in rodent models. ${ }^{23,24}$

Consequently, recently developed implants have been functionalized with proangiogenic compounds, such as growth factors, fibrin and platelet-rich plasma, which mimic the extracellular matrix environment around the implantation site. ${ }^{25-27}$ As many devices include an inlet, prevascularization is an attractive strategy. The device, loaded with proangiogenic compounds, is implanted for four weeks to allow for vascularization before filling with islets via the inlet. ${ }^{28,29}$ During this time, a continued presence of proangiogenic compounds is critical for stable blood vessel formation. ${ }^{30}$ 


\section{Sustained release of growth factors}

Growth factors are of special interests as proangiogenic compounds. Growth factors released from devices stimulate blood vessel growth towards the device by binding to growth factor receptors present on endothelial cells of surrounding blood vessels. ${ }^{31}$ This stimulates their proliferation, migration and tube formation along the growth factor gradient. ${ }^{32,33}$ Vascular endothelial growth factor (VEGF), as one of the most prominent angiogenic growth factors, ${ }^{34,}$ 35 has frequently been used as vascularizing agent for biomaterial implants, ${ }^{26,36}$ as well as fibroblast growth factor ${ }^{33,37-39}$ (FGF) and insulin-like growth factor ${ }^{40,41}$ (IGF-1). However, growth factors have a reported short half-life and are prone to enzymatic degradation, which prevents a continuous week-long presence. ${ }^{42,43}$

Encapsulating growth factors in drug delivery systems can overcome this limitation by providing local sustained release of encapsulated cargo within an intended time frame. Polymeric systems such as hydrogels and microspheres have been developed for sustained release of VEGF and other growth factors. ${ }^{44-46}$ For these systems, the release profile, i.e. sustained release for 4 weeks, and stability of the protein during preparation and release, i.e. release of bioactive growth factor, are of great importance.

Many reported microsphere formulations are based on poly(lactic-co-glycolic acid) (PLGA), a well-characterized and widely applied polymer basis for protein delivery. ${ }^{47-49}$ However, the release from PLGA-based microspheres is often biphasic, i.e. a burst release followed by a lag phase before the onset of polymer degradation, ${ }^{50,51}$ and is as such not favorable for vascularization. ${ }^{24,30}$ Also, the accumulation of acidic degradation products during polymer degradation, causing a decrease in $\mathrm{pH}$ can negatively affect the encapsulated protein's stability. ${ }^{47,52,53}$

Phase-separated multiblock copolymers are an attractive alternative for achieving tailorable release of proteins from microspheres, ${ }^{54,55}$ as shown for Synbiosys ${ }^{\circ}$ polymers. These polymers are composed of amorphous poly( $\varepsilon$-caprolactone)-poly(ethylene glycol)-poly( $\varepsilon$-caprolactone) [PCL-PEG-PCL] blocks and semi-crystalline poly(L-lactide) [PLLA] blocks. Their hydrophilic nature, caused by the presence of PEG, leads to well-controlled swelling and diffusioncontrolled release of encapsulated proteins. ${ }^{56-59}$

Microspheres are commonly prepared by a double emulsion solvent evaporation method, where a protein solution is emulsified with a polymer-containing organic phase. ${ }^{60}$ The exposure to shear stresses and organic solvents can be detrimental to the protein's stability and bioactivity. ${ }^{47,61}$ Alternatively, proteins can be "post-loaded" in microspheres through ionic interaction with an oppositely charged biopolymer present in the porous polymeric matrix. The avoidance of organic solvents and shear stresses makes this process favorable for protein stability. ${ }^{62,63}$ 
Post-loading is a common strategy for loading growth factors in hydrogels, cell-friendly scaffolds for tissue-engineered constructs. ${ }^{64,65}$ Naturally occurring glycosaminoglycans, such as hyaluronic acid and chondroitin sulfate, can interact with positively charged growth factors through their negative charge. ${ }^{66,67}$ Further, hydrogels can offer diffusion-controlled release of loaded proteins. ${ }^{68}$

\section{Aim and outline of this thesis}

The work described in this thesis is part of the European consortium DRIVE (Diabetes-reversing implants with enhanced viability and long-term efficacy; www.drive-project.eu), which received funding from European Union's Horizon 2020 research and innovation program. The DRIVE consortium, consisting of 15 academic and industrial partners, aimed at developing a novel pancreatic islet encapsulation device, an advanced therapy medicinal product (ATMP) for diabetes type 1 treatment.

As shown in figure 3, the DRIVE device is composed of a semi-permeable silicone pouch in which pancreatic islets are embedded using a biocompatible carrier. In order to increase islet survival, the filling suspension contains perfluorocarbons and VEGF-loaded microspheres. Upon transplantation, oxygen-loaded perfluorocarbons will provide short-term oxygen supply of the islets. VEGF, released from microspheres in a time frame of four weeks, will stimulate angiogenesis toward the device, ensuring sufficient vascularization for long-term islet survival.

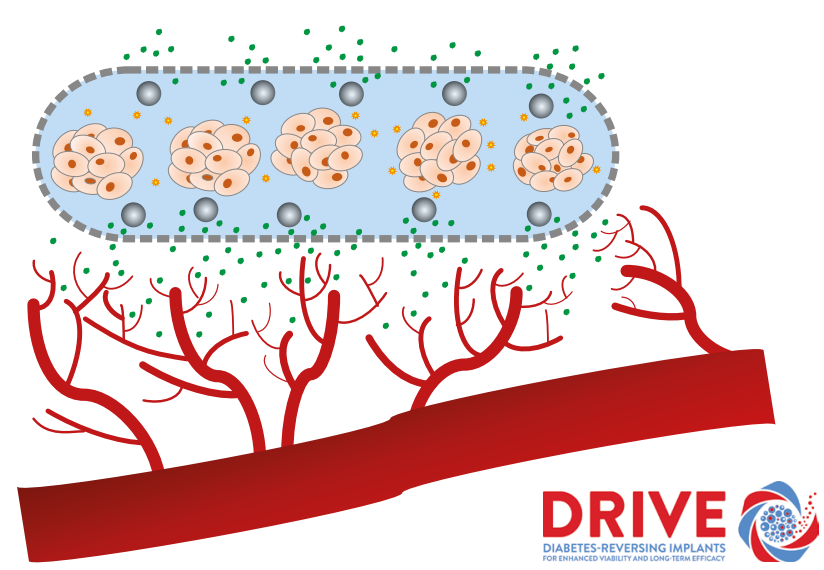

FIGURE 3: Schematic image of the DRIVE pancreatic islet encapsulation device, consisting of a semi-permeable pouch (dashed gray outline). Pancreatic islets (in orange) are embedded using a biocompatible carrier (in blue), together with oxygen precursors (yellow stars) and VEGF-loaded microspheres (gray spheres). VEGF-loaded microspheres release VEGF (in green) in a time period of 4 weeks, thereby stimulating the growth of blood vessels (in red) towards the device. 
The aim of this thesis was to develop polymeric drug delivery systems that exhibit sustained release of VEGF for four weeks. Special emphasis was laid on developing mild, protein-friendly encapsulation methods for microspheres and hydrogels. Furthermore, the bioactivity of released growth factors was addressed with in vitro endothelial cell-based assays.

Chapter 2 describes the preparation of monodisperse VEGF-loaded microspheres based on blends of two [PCL-PEG-PCL]-b-[PLLA] multiblock copolymers. In this chapter, we report the diffusion-controlled release of bioactive VEGF from these microspheres quantified by two analytical methods. Further, we assess the degradation and storage stability of microspheres. In Chapter 3, we report the incorporation of these microspheres in a silicone-based cell macroencapsulation device. We describe a filling procedure, where microspheres are suspended in hyaluronic acid-based carrier liquid and injected into the device's inlet. Herein we focus on the rheological properties of the suspension, the sustained release of VEGF from devices and the bioactivity of released growth factor.

In Chapter 4, we explore a novel, protein-friendly post-loading method for simultaneous loading of growth factors VEGF, FGF and IGF in PLGA microspheres. This method is based on the ionic interaction of positively charged growth factors with negatively charged polysaccharides present in the porous polymeric matrix, which "trap" the growth factors in the microspheres prior to pore closure. We focus on the sustained release of growth factors from the microspheres and have evaluated the bioactivity of released growth factors in light of a synergistic proliferative response of endothelial cells by a combination of growth factors.

In Chapter 5, the ionic interaction of positively charged VEGF with negatively charged polysaccharides is assessed for post-loading VEGF in biocompatible chondroitin sulfate-based hydrogels. We evaluate the effect of co-loading VEGF with the model protein lysozyme, which forms complex precipitates with chondroitin sulfate. We herein focus on the degradation behavior of the hydrogels and the release mechanism of VEGF.

Finally, Chapter $\mathbf{6}$ gives a summary of this thesis' findings and discusses perspectives and implications for future research. 


\section{References}

1. Global report on diabetes; World Health Organization: 2016.

2. Tuomilehto, J. The emerging global epidemic of type 1 diabetes. Curr. Diab. Rep. 2013, 13, (6), 795-804.

3. The DIAMOND Project Group. Incidence and trends of childhood Type 1 diabetes worldwide 1990-1999. Diabet. Med. 2006, 23, (8), 857-66.

4. Ilonen, J.; Lempainen, J.; Veijola, R. The heterogeneous pathogenesis of type 1 diabetes mellitus. Nat. Rev. Endocrinol. 2019, 15, (11), 635-650.

5. Tokarz, V. L.; MacDonald, P. E.; Klip, A. The cell biology of systemic insulin function. J. Cell Biol. 2018, 217, (7), 2273-2289.

6. Tauschmann, M.; Hovorka, R. Technology in the management of type 1 diabetes mellitus - current status and future prospects. Nat. Rev. Endocrinol. 2018, 14, (8), 464-475.

7. Stephens, E. Insulin therapy in type 1 diabetes. Med. Clin. North Am. 2015, 99, (1), 145-56.

8. Malik, F. S.; Taplin, C. E. Insulin therapy in children and adolescents with type 1 diabetes. Paediatr. Drugs 2014, 16, (2), 141-50.

9. Bertuzzi, F; Verzaro, R.; Provenzano, V.; Ricordi, C. Brittle type 1 diabetes mellitus. Curr. Med. Chem. 2007, 14, (16), 1739-44.

10. Cartwright, A.; Wallymahmed, M.; Macfarlane, I. A.; Wallymahmed, A.; Williams, G.; Gill, G. V. The outcome of brittle type 1 diabetes--a 20 year study. QJM 2011, 104, (7), 575-9.

11. Shapiro, A. M.; Pokrywczynska, M.; Ricordi, C. Clinical pancreatic islet transplantation. Nat. Rev. Endocrinol. 2017, 13, (5), 268-277.

12. Shapiro, A. M.; Lakey, J. R.; Ryan, E. A.; Korbutt, G. S.; Toth, E.; Warnock, G. L.; Kneteman, N. M.; Rajotte, R. V. Islet transplantation in seven patients with type 1 diabetes mellitus using a glucocorticoid-free immunosuppressive regimen. N. Engl. J. Med. 2000, 343, (4), 230-8.

13. Shapiro, A. M.; Ricordi, C.; Hering, B. J.; Auchincloss, H.; Lindblad, R.; Robertson, R. P.; Secchi, A.; Brendel, M. D.; Berney, T.; Brennan, D. C.; Cagliero, E.; Alejandro, R.; Ryan, E. A.; DiMercurio, B.; Morel, P.; Polonsky, K. S.; Reems, J. A.; Bretzel, R. G.; Bertuzzi, F; Froud, T.; Kandaswamy, R.; Sutherland, D. E.; Eisenbarth, G.; Segal, M.; Preiksaitis, J.; Korbutt, G. S.; Barton, F. B.; Viviano, L.; Seyfert-Margolis, V.; Bluestone, J.; Lakey, J. R. International trial of the Edmonton protocol for islet transplantation. N. Engl. J. Med. 2006, 355, (13), 1318-30.

14. Khosravi-Maharlooei, M.; Hajizadeh-Saffar, E.; Tahamtani, Y.; Basiri, M.; Montazeri, L.; Khalooghi, K.; Kazemi Ashtiani, M.; Farrokhi, A.; Aghdami, N.; Sadr Hashemi Nejad, A.; Larijani, M. B.; De Leu, N.; Heimberg, H.; Luo, X.; Baharvand, H. Islet transplantation for type 1 diabetes: so close and yet so far away. Eur. J. Endocrinol. 2015, 173, (5), R165-83.

15. Diabetes Research Institute, University of Miami https://www.diabetesresearch.org/images-video (23 October 2019),

16. Dimitrioglou, N.; Kanelli, M.; Papageorgiou, E.; Karatzas, T.; Hatziavramidis, D. Paving the way for successful islet encapsulation. Drug Discov. Today 2019, 24, (3), 737-748.

17. Desai, T.; Shea, L. D. Advances in islet encapsulation technologies. Nat. Rev. Drug Discov. 2017, 16, (5), 338-350.

18. Ryan, A. J.; O'Neill, H. S.; Duffy, G. P.; O'Brien, F. J. Advances in polymeric islet cell encapsulation technologies to limit the foreign body response and provide immunoisolation. Curr. Opin. Pharmacol. 2017, 36, 66-71.

19. Canibano-Hernandez, A.; Saenz Del Burgo, L.; Espona-Noguera, A.; Ciriza, J.; Pedraz, J. L. Current advanced therapy cell-based medicinal products for type-1-diabetes treatment. Int. J. Pharm. 2018, 543, (1-2), 107-120.

20. Yu, T. T. L.; Gupta, P.; Ronfard, V.; Vertes, A. A.; Bayon, Y. Recent progress in European advanced therapy medicinal products and beyond. Front Bioeng Biotechno/ 2018, 6, 130.

21. Bowers, D. T.; Song, W.; Wang, L. H.; Ma, M. Engineering the vasculature for islet transplantation. Acta Biomater. 2019, 95, 131-151.

22. Novosel, E. C.; Kleinhans, C.; Kluger, P. J. Vascularization is the key challenge in tissue engineering. Adv Drug Deliv $\operatorname{Rev}$ 2011, 63, (4-5), 300-11.

23. Silva, E. A.; Mooney, D. J. Effects of VEGF temporal and spatial presentation on angiogenesis. Biomaterials 2010, $31,(6), 1235-41$. 
24. Davies, N.; Dobner, S.; Bezuidenhout, D.; Schmidt, C.; Beck, M.; Zisch, A. H.; Zilla, P. The dosage dependence of VEGF stimulation on scaffold neovascularisation. Biomaterials 2008, 29, (26), 3531-8.

25. Farina, M.; Chua, C. Y. X.; Ballerini, A.; Thekkedath, U.; Alexander, J. F.; Rhudy, J. R.; Torchio, G.; Fraga, D.; Pathak, R. R.; Villanueva, M.; Shin, C. S.; Niles, J. A.; Sesana, R.; Demarchi, D.; Sikora, A. G.; Acharya, G. S.; Gaber, A. O.; Nichols, J. E.; Grattoni, A. Transcutaneously refillable, 3D-printed biopolymeric encapsulation system for the transplantation of endocrine cells. Biomaterials 2018, 177, 125-138.

26. Farina, M.; Ballerini, A.; Fraga, D. W.; Nicolov, E.; Hogan, M.; Demarchi, D.; Scaglione, F.; Sabek, O. M.; Horner, P.; Thekkedath, U.; Gaber, O. A.; Grattoni, A. 3D printed vascularized device for subcutaneous transplantation of human islets. Biotechnol J 2017, 12, (9).

27. Smink, A. M.; Li, S.; Hertsig, D. T.; de Haan, B. J.; Schwab, L.; van Apeldoorn, A. A.; de Koning, E.; Faas, M. M.; Lakey, J. R.; de Vos, P. The efficacy of a prevascularized, retrievable poly(D,L,-lactide-co-epsilon-caprolactone) subcutaneous scaffold as transplantation site for pancreatic islets. Transplantation 2017, 101, (4), e112-e119.

28. Uematsu, S. S.; Inagaki, A.; Nakamura, Y.; Imura, T.; Igarashi, Y.; Fathi, I.; Miyagi, S.; Ohuchi, N.; Satomi, S.; Goto, M. The optimization of the prevascularization procedures for improving subcutaneous islet engraftment. Transplantation 2018, 102, (3), 387-395.

29. Laschke, M. W.; Menger, M. D. Prevascularization in tissue engineering: Current concepts and future directions. Biotechnol. Adv. 2016, 34, (2), 112-21.

30. Amsden, B. G.; Timbart, L.; Marecak, D.; Chapanian, R.; Tse, M. Y.; Pang, S. C. VEGF-induced angiogenesis following localized delivery via injectable, low viscosity poly(trimethylene carbonate). J. Control. Release 2010, 145, (2), 109-15.

31. Rouwkema, J.; Rivron, N. C.; van Blitterswijk, C. A. Vascularization in tissue engineering. Trends Biotechnol. 2008, 26, (8), 434-41.

32. Uccelli, A.; Wolff, T.; Valente, P.; Di Maggio, N.; Pellegrino, M.; Gurke, L.; Banfi, A.; Gianni-Barrera, R. Vascular endothelial growth factor biology for regenerative angiogenesis. Swiss Med. Wkly. 2019, 149, w20011.

33. Carmeliet, P.; Jain, R. K. Molecular mechanisms and clinical applications of angiogenesis. Nature 2011, 473, (7347), 298-307.

34. Yla-Herttuala, S.; Rissanen, T. T.; Vajanto, I.; Hartikainen, J. Vascular endothelial growth factors: biology and current status of clinical applications in cardiovascular medicine. J. Am. Coll. Cardiol. 2007, 49, (10), 1015-26.

35. Ferrara, N.; Gerber, H. P.; LeCouter, J. The biology of VEGF and its receptors. Nat. Med. 2003, 9, (6), 669-76.

36. Wagner, E. R.; Parry, J.; Dadsetan, M.; Bravo, D.; Riester, S. M.; Van Wijnen, A. J.; Yaszemski, M. J.; Kakar, S. VEGFmediated angiogenesis and vascularization of a fumarate-crosslinked polycaprolactone (PCLF) scaffold. Connect. Tissue Res. 2018, 59, (6), 542-549.

37. Cross, M. J.; Claesson-Welsh, L. FGF and VEGF function in angiogenesis: signalling pathways, biological responses and therapeutic inhibition. Trends Pharmacol. Sci. 2001, 22, (4), 201-7.

38. Kitamura, N.; Hasebe, T.; Matsumoto, T.; Hotta, A.; Suzuki, T.; Yamagami, T.; Terada, H. Basic fibroblast growth factor as a potential stent coating material inducing endothelial cell proliferation. J Atheroscler Thromb 2014, 21, (5), 477-485.

39. Montero, R. B.; Vial, X.; Nguyen, D. T.; Farhand, S.; Reardon, M.; Pham, S. M.; Tsechpenakis, G.; Andreopoulos, F. M. bFGF-containing electrospun gelatin scaffolds with controlled nano-architectural features for directed angiogenesis. Acta Biomater. 2012, 8, (5), 1778-91.

40. Bach, L. A. Endothelial cells and the IGF system. J. Mol. Endocrinol. 2015, 54, (1), R1-13.

41. Cittadini, A.; Monti, M. G.; Petrillo, V.; Esposito, G.; Imparato, G.; Luciani, A.; Urciuolo, F.; Bobbio, E.; Natale, C. F.; Sacca, L.; Netti, P. A. Complementary therapeutic effects of dual delivery of insulin-like growth factor-1 and vascular endothelial growth factor by gelatin microspheres in experimental heart failure. Eur. J. Heart Fail. 2011, 13, (12), 1264-74.

42. Kleinheinz, J.; Jung, S.; Wermker, K.; Fischer, C.; Joos, U. Release kinetics of VEGF165 from a collagen matrix and structural matrix changes in a circulation model. Head Face Med. 2010, 6, 17.

43. Morishita, M.; Peppas, N. A. Is the oral route possible for peptide and protein drug delivery? Drug Discov. Today 2006, 11, (19-20), 905-10. 
44. Cleland, J. L.; Duenas, E. T.; Park, A.; Daugherty, A.; Kahn, J.; Kowalski, J.; Cuthbertson, A. Development of poly(D,L-lactide--coglycolide) microsphere formulations containing recombinant human vascular endothelial growth factor to promote local angiogenesis. J. Control. Release 2001, 72, (1-3), 13-24.

45. Phelps, E. A.; Templeman, K. L.; Thule, P. M.; Garcia, A. J. Engineered VEGF-releasing PEG-MAL hydrogel for pancreatic islet vascularization. Drug Deliv Trans/ Res 2015, 5, (2), 125-36.

46. Zieris, A.; Prokoph, S.; Levental, K. R.; Welzel, P. B.; Grimmer, M.; Freudenberg, U.; Werner, C. FGF-2 and VEGF functionalization of starPEG-heparin hydrogels to modulate biomolecular and physical cues of angiogenesis. Biomaterials 2010, 31, (31), 7985-94.

47. van de Weert, M.; Hennink, W. E.; Jiskoot, W. Protein instability in poly(lactic-co-glycolic acid) microparticles. Pharm. Res. 2000, 17, (10), 1159-67.

48. Giteau, A.; Venier-Julienne, M. C.; Aubert-Pouessel, A.; Benoit, J. P. How to achieve sustained and complete protein release from PLGA-based microparticles? Int. J. Pharm. 2008, 350, (1-2), 14-26.

49. Schwendeman, S. P. Recent advances in the stabilization of proteins encapsulated in injectable PLGA delivery systems. Crit. Rev. Ther. Drug Carrier Syst. 2002, 19, (1), 73-98.

50. Rui, J.; Dadsetan, M.; Runge, M. B.; Spinner, R. J.; Yaszemski, M. J.; Windebank, A. J.; Wang, H. Controlled release of vascular endothelial growth factor using poly-lactic-co-glycolic acid microspheres: in vitro characterization and application in polycaprolactone fumarate nerve conduits. Acta Biomater. 2012, 8, (2), 511-8.

51. Simon-Yarza, T.; Tamayo, E.; Benavides, C.; Lana, H.; Formiga, F. R.; Grama, C. N.; Ortiz-de-Solorzano, C.; Kumar, M. N.; Prosper, F.; Blanco-Prieto, M. J. Functional benefits of PLGA particulates carrying VEGF and CoQ10 in an animal of myocardial ischemia. Int. J. Pharm. 2013, 454, (2), 784-90.

52. Fu, K.; Pack, D. W.; Klibanov, A. M.; Langer, R. Visual evidence of acidic environment within degrading poly(lacticco-glycolic acid) (PLGA) microspheres. Pharm. Res. 2000, 17, (1), 100-6.

53. Shenderova, A.; Burke, T. G.; Schwendeman, S. P. The acidic microclimate in poly(lactide-co-glycolide) microspheres stabilizes camptothecins. Pharm. Res. 1999, 16, (2), 241-8.

54. Kissel, T.; Li, Y.; Unger, F. ABA-triblock copolymers from biodegradable polyester A-blocks and hydrophilic poly(ethylene oxide) B-blocks as a candidate for in situ forming hydrogel delivery systems for proteins. Adv Drug Deliv Rev 2002, 54, (1), 99-134.

55. Bonacucina, G.; Cespi, M.; Mencarelli, G.; Giorgioni, G.; Palmieri, G. F. Thermosensitive self-assembling block copolymers as drug delivery systems. Polymers-Basel 2011, 3, (2), 779-811.

56. Stankovic, M.; de Waard, H.; Steendam, R.; Hiemstra, C.; Zuidema, J.; Frijlink, H. W.; Hinrichs, W. L. Low temperature extruded implants based on novel hydrophilic multiblock copolymer for long-term protein delivery. Eur. J. Pharm. Sci. 2013, 49, (4), 578-87.

57. Stankovic, M.; Hiemstra, C.; de Waard, H.; Zuidema, J.; Steendam, R.; Frijlink, H. W.; Hinrichs, W. L. Protein release from water-swellable poly(D,L-lactide-PEG)-b-poly(-caprolactone) implants. Int. J. Pharm. 2015, 480, (1-2), 7383.

58. Stankovic, M.; Tomar, J.; Hiemstra, C.; Steendam, R.; Frijlink, H. W.; Hinrichs, W. L. Tailored protein release from biodegradable poly(epsilon-caprolactone-PEG)-b-poly(epsilon-caprolactone) multiblock-copolymer implants. Eur. J. Pharm. Biopharm. 2014, 87, (2), 329-37.

59. Sandker, M. J.; Duque, L. F.; Redout, E. M.; Chan, A.; Que, I.; Lowik, C. W.; Klijnstra, E. C.; Kops, N.; Steendam, R.; van Weeren, R.; Hennink, W. E.; Weinans, H. Degradation, intra-articular retention and biocompatibility of monospheres composed of [PDLLA-PEG-PDLLA]-b-PLLA multi-block copolymers. Acta Biomater. 2017, 48, 401414.

60. Iqbal, M.; Zafar, N.; Fessi, H.; Elaissari, A. Double emulsion solvent evaporation techniques used for drug encapsulation. Int. J. Pharm. 2015, 496, (2), 173-90.

61. Thomas, C. R.; Geer, D. Effects of shear on proteins in solution. Biotechnol. Lett. 2011, 33, (3), 443-56.

62. Reinhold, S. E.; Desai, K. G.; Zhang, L.; Olsen, K. F.; Schwendeman, S. P. Self-healing microencapsulation of biomacromolecules without organic solvents. Angew. Chem. Int. Ed. Engl. 2012, 51, (43), 10800-3.

63. Shah, R. B.; Schwendeman, S. P. A biomimetic approach to active self-microencapsulation of proteins in PLGA. J. Control. Release 2014, 196, 60-70.

64. Malda, J.; Visser, J.; Melchels, F. P.; Jungst, T.; Hennink, W. E.; Dhert, W. J.; Groll, J.; Hutmacher, D. W. 25th anniversary article: Engineering hydrogels for biofabrication. Adv Mater 2013, 25, (36), 5011-28. 
65. Buwalda, S. J.; Boere, K. W.; Dijkstra, P. J.; Feijen, J.; Vermonden, T.; Hennink, W. E. Hydrogels in a historical perspective: from simple networks to smart materials. J. Control. Release 2014, 190, $254-73$.

66. Lim, D. K.; Wylie, R. G.; Langer, R.; Kohane, D. S. Selective binding of C-6 OH sulfated hyaluronic acid to the angiogenic isoform of VEGF(165). Biomaterials 2016, 77, 130-138.

67. Schuurmans, C. C. L.; Abbadessa, A.; Bengtson, M. A.; Pletikapic, G.; Eral, H. B.; Koenderink, G.; Masereeuw, R.; Hennink, W. E.; Vermonden, T. Complex coacervation-based loading and tunable release of a cationic protein from monodisperse glycosaminoglycan microgels. Soft Matter 2018, 14, (30), 6327-6341.

68. Censi, R.; Di Martino, P.; Vermonden, T.; Hennink, W. E. Hydrogels for protein delivery in tissue engineering. J. Control. Release 2012, 161, (2), 680-92. 



\title{
Chapter 2
}

\section{Sustained Release of Vascular Endothelial Growth Factor from Poly( $\varepsilon$-caprolactone-PEG- $\varepsilon$-caprolactone)-b-poly(L-lactide) Multiblock Copolymer Microspheres}

\author{
Karina C. Scheiner', Roel F. Maas-Bakker', Thanh T. Nguyen², \\ Ana M. Duarte ${ }^{2}$, Gert Hendriks² ${ }^{2}$ Lídia Sequeira ${ }^{2}$, Garry P. Duffy ${ }^{3}$, \\ Rob Steendam², Wim E. Hennink', Robbert ]. Kok', \\ ' Department of Pharmaceutics, Utrecht Institute for Pharmaceutical Sciences, Utrecht \\ University, Universiteitsweg 99, 3584 CG Utrecht, the Netherlands \\ ${ }^{2}$ InnoCore Pharmaceuticals B.V., L.J. Zielstraweg 1, 9713 GX Groningen, the Netherlands \\ ${ }^{3}$ Discipline of Anatomy, School of Medicine, National University of Ireland Galway, University \\ Road, Galway, Ireland
}




\section{Abstract}

Vascular endothelial growth factor (VEGF) is the major regulating factor for the formation of new blood vessels, also known as angiogenesis. VEGF is often incorporated in synthetic scaffolds to promote vascularization and to enhance the survival of cells that have been seeded in these devices. Such applications require sustained local delivery of VEGF of around four weeks for stable blood vessel formation. Most delivery systems for VEGF only provide short-term release for a couple of days, followed by a release phase with very low VEGF release. We now have developed VEGF-loaded polymeric microspheres that provide sustained release of bioactive VEGF for four weeks. Blends of two swellable poly( $\varepsilon$-caprolactone)-poly(ethylene glycol)-poly( $\varepsilon$-caprolactone)-b-poly(L-lactide) ([PCL-PEG-PCL]-b-[PLLA])-based multiblock copolymers with different PEG content and PEG molecular weight were used to prepare the microspheres. Loading of the microspheres was established by a solvent evaporation-based membrane emulsification method. The resulting VEGF-loaded microspheres had average sizes of $40-50 \mu \mathrm{m}$ and a narrow size distribution. Optimized formulations of a 50:50 blend of the two multiblock copolymers had an average VEGF loading of $0.79 \pm 0.09 \%$, representing a high average VEGF loading efficiency of $78 \pm 16 \%$. These microspheres released VEGF continuously over four weeks in PBS pH 7.4 at $37{ }^{\circ} \mathrm{C}$. This release profile was preserved after repeated and long-term storage at $-20^{\circ} \mathrm{C}$ for up to 9 months, thereby demonstrating excellent storage stability. VEGF release was governed by diffusion through the water-filled polymer matrix, depending on PEG molecular weight and PEG content of the polymers. The bioactivity of the released VEGF was retained within the experimental error in the four-week release window, as demonstrated using a HUVEC proliferation assay. Thus, the microspheres prepared in this study are suitable for embedment in polymeric scaffolds with the aim of promoting their functional vascularization.
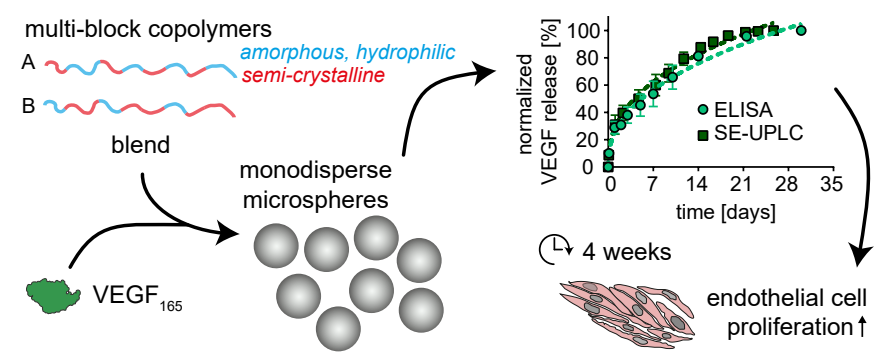


\section{Introduction}

Tissue engineering aims at developing bioartificial implants which can be used for replacement or repair of damaged tissues. ${ }^{1-3}$ Different categories of such engineered tissues can be distinguished: full synthetic biomaterials, (decellularized) scaffolds from natural origins and hybrid biomaterials that consist of both synthetic and biological or cellular components. ${ }^{4-6}$ When the implanted material contains cells or when it will be infiltrated by cells after it has been transplanted, one can consider such biomaterial as an artificial tissue or organ. ${ }^{7}$ One of the major challenges of constructing cellularized biomaterials is the sufficient supply of nutrients and oxygen from the systemic circulation into the implant. ${ }^{8}$ Incorporation of growth factors into the tissue-engineered construct can locally stimulate angiogenesis towards the implant, thereby providing vascularization of the construct.9, 10 Vascular endothelial growth factor (VEGF) is the most prominent proangiogenic growth factor and stimulates angiogenesis by binding to the VEGF receptor present on endothelial cells. ${ }^{11}$ Stable vascularization of an implant depends on the dose of VEGF and the time period of VEGF release at the site of implantation. 10,12 Current literature suggests that a constant dose of VEGF for around 4 weeks leads to sufficient vascularization in rodent models. ${ }^{13,14}$ Formulation of VEGF into a drug delivery system ideally offers control of the amount of VEGF released and the time span of VEGF release into the adjacent tissue. ${ }^{15,16}$ Drug delivery systems such as hydrogels, viscous liquids and microspheres have been developed for sustained release of VEGF.17-19 Particularly polymer-based microspheres present an attractive delivery platform for proteins due to their tailorable polymer-dependent release profile and enhanced stability of the encapsulated cargo. ${ }^{20-22}$ Several groups have formulated VEGF in microspheres based on PLGA, which is the most widely applied polymer used for the design for controlled release formulations. ${ }^{23-27}$ Release of VEGF from PLGA matrices, however, is often biphasic with a high initial burst within the first few hours followed by a relative slow continuous release in the following weeks. ${ }^{28-30}$ This release profile is not ideal for functional vascularization of biomaterials as a more sustained elevation of VEGF is needed for the stimulation of blood vessel growth.14,

${ }^{31}$ Commonly, burst release from PLGA-based microspheres is not controlled by erosion of the polymer particles, but by surface-bound cargo and protein molecules that are released from pores present in the particles that allow rapid diffusion and release of biopharmaceutical drugs. $^{32,33}$ Another limitation of PLGA-based systems is the retention of acidic degradation products within the polymeric matrix, resulting in a $\mathrm{pH}$ decrease in the microspheres ${ }^{34,35}$ that can have a negative impact on the stability of proteins. ${ }^{23,36}$ In order to obtain tailorable release of biopharmaceutical protein drugs, Kazazi-Hyseni et al. prepared PLGA microspheres with differences in porosity by varying formulation parameters such as polymer concentration and oil/water ratios in a membrane emulsification process. ${ }^{37}$ An alternative approach for tailoring the release of pharmaceutical proteins from polymeric microspheres is the use of phase-separated multiblock copolymers. ${ }^{38,} 39$ SynBiosys ${ }^{\circ}$ multiblock copolymers composed 
of amorphous poly( $\varepsilon$-caprolactone) - poly(ethylene glycol) - poly( $\varepsilon$-caprolactone) (PCL-PEG$P C L$ ) blocks and semi-crystalline poly(L-lactic acid) (PLLA) blocks (as shown in figure 1) are more hydrophilic than for example PLGA, due to the presence of PEG. The well-controlled swelling of these [PCL-PEG-PCL]-b-[PLLA] polymers allows continuous release of encapsulated molecules through diffusion with low burst release. ${ }^{40-42}$ The swelling degree of these [PCLPEG-PCL]-b-[PLLA] polymers is dependent on the weight fraction and molecular weight of PEG. ${ }^{42,43}$ We hypothesized that release characteristics of VEGF from multiblock copolymeric microspheres can be tailored by blending multiblock copolymers with different swelling degree.

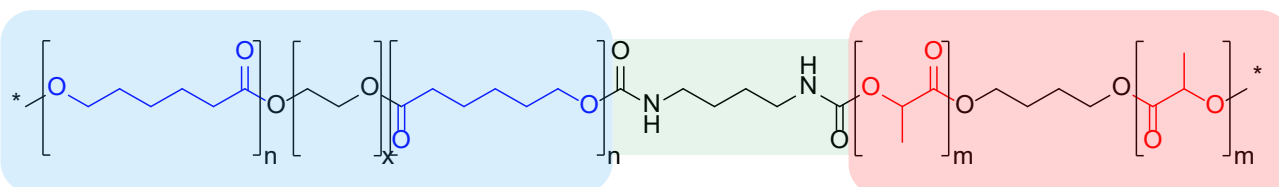

FIGURE 1: General chemical composition of multiblock copolymers used in this study. In blue shading: amorphous, hydrophilic block, n: poly(E-caprolactone), x: poly(ethylene glycol). In green shading: urethane linker, in red shading: semi-crystalline block, m: poly(L-lactic acid). The two polymers used in this study differ in their block weight fraction (hydrophilic block - semi-crystalline block), PEG chain length and total PEG weight fraction.

In the present study, VEGF was formulated in multiblock copolymer microspheres using a double emulsion-based membrane emulsification method. With this method, monodisperse microspheres with a narrow, well-defined size distribution are obtained, which is a determining parameter for well-defined and reproducible controlled release systems. ${ }^{44,45}$ Furthermore, membrane emulsification is advantageous due to its low batch-to-batch variability, decreased mechanical stress on the loaded protein and high encapsulation efficiency. ${ }^{21,46,47}$ To our knowledge, this is the first occasion in which monodisperse VEGF-loaded microparticles are prepared by membrane emulsification, in which we furthermore aim at a sustained release profile of several weeks. The release of VEGF from microspheres was measured by two analytical methods, namely SE-UPLC and ELISA. Finally, the bioactivity of VEGF released during the entire release period was assessed by a HUVEC proliferation assay.

\section{Experimental section}

\section{Materials}

Recombinant human VEGF 165 was purchased from Peprotech Inc. (Rocky Hill, NJ, USA). Polyvinyl alcohol (PVA 5-88, 85-89\% hydrolyzed) and sodium chloride ( $\mathrm{NaCl}$ ) were purchased from Merck (Germany). Disodium succinate, sodium dodecyl sulphate (SDS), trichloroacetylisocyanate 
(TCAI) and bovine serum albumin (BSA) were purchased from Sigma Aldrich (Germany). Dimethyl sulfoxide (DMSO), Tween 20, sodium azide, potassium dihydrogen phosphate $\left(\mathrm{KH}_{2} \mathrm{PO}_{4}\right)$ and dipotassium hydrogen phosphate $\left(\mathrm{Na}_{2} \mathrm{HPO}_{4}\right)$ were purchased from Fisher Scientific (The Netherlands). Sodium hydroxide $(\mathrm{NaOH})$ was purchased from WWR (The Netherlands). Gibco Dulbecco's Phosphate Buffered Saline (DPBS, 10x, composition 27 mM $\mathrm{KCl}, 15 \mathrm{mM} \mathrm{H}_{2} \mathrm{PO}_{4^{\prime}} 1.4 \mathrm{M} \mathrm{NaCl}, 81 \mathrm{mM} \mathrm{Na}_{2} \mathrm{HPO}_{4} \times 7 \mathrm{H}_{2} \mathrm{O}$ ) was purchased from Thermo Fisher Scientific (The Netherlands). PEG standards for GPC analysis were purchased from PSS Polymer Standards Services (Germany).

\section{Methods}

\section{Polymer synthesis and characterization}

Multiblock copolymers A and B (general chemical composition shown in figure 1) were synthesized and characterized based on procedures described by Stankovic et al. and Teekamp et al.. ${ }^{40,48}$ Polymer A consisted of 30 wt\% of an amorphous, hydrophilic poly( $(\varepsilon$-caprolactone)$\mathrm{PEG}_{3000}$ - poly( $\varepsilon$-caprolactone) (PCL-PEG $3000-\mathrm{PCL}$ ) block with a molecular weight of $4,000 \mathrm{~g} / \mathrm{mol}$ and $70 \mathrm{wt} \%$ of a semi-crystalline poly(L-lactide) (PLLA) block with a molecular weight of 4,000 $\mathrm{g} / \mathrm{mol}$. The total PEG weight fraction of polymer $\mathrm{A}$ is $22.5 \%$. Polymer B consisted of $50 \mathrm{wt} \%$ of PCL-PEG ${ }_{1000}-P C L\left(M_{w}: 2,000 \mathrm{~g} / \mathrm{mol}\right)$ and $50 \mathrm{wt} \%$ of poly(L-lactide) block ( $\left.\mathrm{M}_{\mathrm{w}}: 4,000 \mathrm{~g} / \mathrm{mol}\right)$, resulting in a PEG weight fraction of $25 \%$. The molecular weights of polymer $A$ and $B$ are reported in the supporting information.

\section{Preparation of monodisperse microspheres}

Polymeric microspheres were prepared by a membrane emulsification-based double emulsion method. Table 1 shows the compositions of the different water and organic phases used for the preparation of the different formulations.

TABLE 1: Composition of the water and oil phases used for the preparation of microspheres.

\begin{tabular}{|c|c|c|c|c|}
\hline \multirow{2}{*}{\multicolumn{2}{|c|}{ Formulation parameters }} & \multicolumn{3}{|c|}{ Target VEGF loading } \\
\hline & & placebo & 0.2 wt\% & 1.0 wt\% \\
\hline$w_{1}$-phase & VEGF concentration & n.a.* & $2 \mathrm{mg} / \mathrm{ml}$ & $15-20 \mathrm{mg} / \mathrm{ml}$ \\
\hline \multirow[t]{2}{*}{ o-phase } & Polymer concentration in DCM & $10 w t \%$ & $10 \mathrm{wt} \%$ & $15-20 w t \%$ \\
\hline & Ratio VEGF : polymer & n.a. & $1: 500$ & $1: 100$ \\
\hline$w_{2}$-phase & Continuous phase & $\begin{array}{l}0.4 \text { wt } \% \text { PVA, } \\
5 \text { wt } \% \mathrm{NaCl}\end{array}$ & $\begin{array}{l}0.4 \text { wt } \% \text { PVA, } \\
5 \text { wt } \% \mathrm{NaCl}\end{array}$ & $\begin{array}{l}4 \text { wt } \% \text { PVA, } \\
5 \text { wt } \% \mathrm{NaCl}\end{array}$ \\
\hline
\end{tabular}

${ }^{*}$ n.a. $=$ not applicable 
Polymers $A$ and $B$ at different weight ratios (table 2) were dissolved in dichloromethane (DCM) to yield a 10 - 20 wt\% solution (see table 1). Lyophilized carrier-free VEGF was reconstituted in 5 mM succinate buffer pH 5 (see table 1 for concentrations), added to the polymer solution (final VEGF target loading $0.2 \mathrm{wt} \%$ or $1.0 \mathrm{wt} \%$ versus total mass of polymer/ ratio 1:10 volume $W_{\text {- }}$ phase to volume o-phase) and homogenized for 40 seconds at 21,600 rpm using an Ultra-turrax mixer (T25 Basic, IKA, Wilmington, USA). The obtained primary emulsion was subsequently pressed through a stainless steel membrane with a pore size of $20 \mu \mathrm{m}$ (20 $\mu \mathrm{m}$ x 200 um Hydrophilic Ringed Stainless-Steel Membrane, Micropore Technologies, Redcar, United Kingdom) into a continuous phase consisting of 0.4 wt\% PVA, 5 wt $\% \mathrm{NaCl}$ or 4 wt\% PVA, 5 wt\% NaCl. Next, the secondary emulsion was stirred at $200 \mathrm{rpm}$ with a magnetic stirrer for 3 hours to evaporate DCM. Subsequently, the particles were washed with $0.05 \%$ Tween 80 and water, and were collected on a $5 \mu \mathrm{m}$ filter before lyophilization overnight. After freezedrying, microspheres were stored at $-20^{\circ} \mathrm{C}$. Placebo microspheres were prepared by single emulsion, i.e. pressing a 10 wt\% polymer solution in DCM (polymer A and B blend ratio 50:50) directly through a stainless steel membrane (as described above, for formulation parameters see table 1).

TABLE 2: Polymer A and B blend ratios and calculated PEG content [\%].

\begin{tabular}{ccccc}
\hline $\begin{array}{c}\text { Weight fraction } \\
\text { Polymer A }\end{array}$ & $\begin{array}{c}\text { Weight fraction } \\
\text { Polymer B }\end{array}$ & PEG 1 kDa [wt\%] & PEG 3 kDa [wt\%] & Total PEG [wt\%] \\
\hline 100 & 0 & 0.0 & 22.5 & 22.5 \\
90 & 10 & 2.5 & 20.3 & 22.8 \\
80 & 20 & 5.0 & 18.0 & 23.0 \\
70 & 30 & 7.5 & 15.8 & 23.3 \\
60 & 40 & 10.0 & 13.5 & 23.5 \\
$\mathbf{5 0}$ & $\mathbf{5 0}$ & $\mathbf{1 2 . 5}$ & $\mathbf{1 1 . 3}$ & $\mathbf{2 3 . 8}$ \\
0 & 100 & 25.0 & 0.0 & 25.0 \\
\hline
\end{tabular}

Blend ratio 50:50 was selected for preparation of placebo microspheres and microspheres with a 1.0 wt\% target VEGF loading (in bold).

\section{Characterization of microspheres}

Average size and size distribution of the obtained microspheres were measured with an optical particle sizer (Accusizer 780, Santa Barbara, California, USA for placebo microspheres and 0.2 wt\% target loading microspheres; Coulter Counter, Beckman Coulter, Woerden, the Netherlands for $1.0 \mathrm{wt} \%$ target loading microspheres). The volume-weight mean microsphere diameter (vol-wt mean) is reported as particle size. The coefficient of variance (CV) [\%] was calculated by dividing the standard deviation by the mean diameter, according to equation 1. 


$$
C V=\frac{\text { standard deviation }[\mu \mathrm{m}]}{\text { meandiameter }[\mu \mathrm{m}]} \times 100 \%
$$

EQUATION 1: Coefficient of variance [\%]

The morphology of the microspheres was analyzed with scanning electron microscopy (SEM, Phenom, FEl Company, the Netherlands or JEOL JCM-5000 Neoscope, JEOL Ltd., Japan). Lyophilized microspheres were transferred onto aluminum specimen stubs using doublesided adhesive tape (Agar Scientific Ltd., England or JEOL Ltd., Japan). Prior to analysis, the microspheres were coated with a thin metal layer using an ion coater under vacuum. The sample was then introduced into the microscope, vacuum was applied and the microspheres were imaged using an electron beam.

VEGF loading and encapsulation efficiency was determined by dissolving $10 \mathrm{mg}$ of microspheres in $0.5 \mathrm{ml}$ of DMSO. The samples were heated at $80^{\circ} \mathrm{C}$ until complete dissolution. Subsequently, $2.5 \mathrm{ml}$ of $0.05 \mathrm{M} \mathrm{NaOH}$ containing $0.5 \%$ SDS was added to each sample, similar to Sah et al. and Ghassemi et al..49,50 Samples were incubated overnight at room temperature for 16 hours under gentle agitation. Subsequently, $100 \mu \mathrm{l}$ of sample was transferred into a glass tube and analyzed for protein content by BCA protein assay (Fisher Scientific, the Netherlands). The amount of VEGF per sample was calculated using a calibration curve of VEGF. Known amounts of VEGF in solution were transferred into glass tubes and evaporated to dryness. These tubes were further treated as samples, as described above. Addition of polymer to the calibration standards did not affect the accuracy of the method (data not shown). VEGF loading is expressed as mg VEGF encapsulated per mg of solid material in weight percentage (wt\%) (equation 2). Loading efficiency was calculated by dividing the actual loading of VEGF by the initial weight \% of VEGF versus the total dry weight of added materials \% [\%] (equation 3).

$$
\begin{gathered}
\text { Loading }[w t \%]=\frac{\text { encapsulated VEGF in microspheres }[\mathrm{mg}]}{\text { dry weight microspheres }[\mathrm{mg}]} \times 100 \% \\
\text { EQUATION 2: Loading [\%] }
\end{gathered}
$$

$$
\text { Loading efficiency }[\%]=\frac{\text { measured VEGF loading }[w t \%]}{\text { theoretical VEGF loading }[w t \%]} \times 100 \%
$$

EQUATION 3: Loading efficiency [\%]

\section{In vitro polymer degradation}

The degradation of placebo microspheres of a 50:50 blend of polymer A and B was studied by suspending $10 \mathrm{mg}$ of accurately weighed freeze-dried microspheres in $1 \mathrm{ml}$ of in vitro release (IVR) buffer (Dulbecco's PBS pH 7.4 (0.2 $\mu \mathrm{m}$ filtered), 0.025\% Tween 20, 0.02\% $\mathrm{NaN}_{3}$ ) 
in $1.5 \mathrm{ml}$ Eppendorf tubes. Samples were incubated at $37^{\circ} \mathrm{C}$ for $7,14,21$ or 28 days under gentle agitation. At these time points, samples were centrifuged at $2000 \mathrm{~g}$ for 5 minutes. After removal of the supernatant, the microspheres were washed three times with $900 \mu \mathrm{l}$ reverse osmosis water and subsequently freeze-dried overnight. The weight of freeze-dried microspheres was noted, and the remaining dry mass per time point was calculated with equation 4, essentially as described previously. ${ }^{42,43,51}$

$$
\text { remaining drymass }[\%]=\frac{m_{d r y, t}}{m_{d r y, 0}} \times 100 \%
$$

EQUATION 4: Calculation of remaining dry mass, where $m_{d r y, 0}$ and $m_{d r y, t}$ are the weights of dry samples at day 0 and dry sample at day t.

The remaining PEG content of degraded samples was determined by ${ }^{1} \mathrm{H}-\mathrm{NMR}$ analysis, as described by Sandker et al. and Ramazani et al.. ${ }^{43,511} \mathrm{H}$ NMR samples were prepared by dissolving approximately $5 \mathrm{mg}$ of degraded microspheres in $0.6 \mathrm{ml}$ of deuterated chloroform $\left(\mathrm{CDCl}_{3}\right)$. The spectrum was recorded from 0 to 8 ppm with an Agilent $400 \mathrm{MHz}$ NMR spectrometer (Agilent Technologies, USA) at 298K. The weight percentage of PEG in degraded polymers was calculated from the methine group of L-lactide at $\delta 5.4-5.1$ and the methylene groups of PEG $\delta$ 3.6-3.7. The molar composition of degradation study samples was further studied by adding the shift reagent trichloroacetylisocyanate (TAIC) to the samples, as described previously by Petit et al. and by de Vos et al...52,53 After reaction of free hydroxyl-end group of PEG with TAIC, the methylene group next to the urethane-containing end group shifts around 1 ppm higher, i.e. $\sim \delta 4.5$, than that of integrated PEG.

Gel permeation chromatography (GPC) was used to analyze the molecular weights of degraded polymers. GPC was carried out on a Waters Alliance system, with a Waters 2695 separating module and a Waters 2414 Refractive Index detector. Two PL-gel 5 Hm mixed-D columns fitted with a guard column (Polymer Labs, $M_{w}$ range $0.2-400 \mathrm{~kg} / \mathrm{mol}$ ) were used. Mobile phase consisted of DMF supplemented with $10 \mathrm{mM} \mathrm{LiCl}$ and calibration was done with PEG standards.

\section{In vitro release studies}

Release studies with VEGF-loaded microspheres were performed at $37^{\circ} \mathrm{C}$. In vitro release

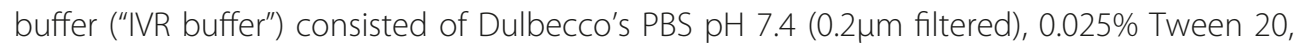
$0.02 \% \mathrm{NaN}_{3}$. Accurately weighed samples of $10 \mathrm{mg}$ lyophilized microspheres were transferred into $1.5 \mathrm{ml}$ Eppendorf tubes and suspended in $900 \mu$ l of IVR buffer. Samples were incubated at $37^{\circ} \mathrm{C}$ under gentle agitation in a rotating shaker. At predetermined time points, samples were centrifuged at $2000 \mathrm{~g}$ for 5 minutes and $800 \mu \mathrm{l}$ of supernatant was removed and replaced by fresh IVR buffer. Samples were stored at $4{ }^{\circ} \mathrm{C}$ until analysis of VEGF content by size exclusion ultra-performance liquid chromatography (SE-UPLC) and enzyme-linked immunosorbent assay (ELISA) as described below. 
Similarly performed release studies from microspheres stored at $-20^{\circ} \mathrm{C}$ for several months (and exposed to several freezing cycles) were conducted in sterilized release buffer ("bioactivity IVR buffer") that did not contain sodium azide and Tween 20. This buffer consisted of Dulbecco's PBS pH 7.4, 0.2 $\mu \mathrm{m}$ filtered (as stated above), 0.5\% BSA, $30 \mu \mathrm{g} / \mathrm{ml}$ gentamicin and $15 \mathrm{ng} / \mathrm{ml}$ amphotericin. VEGF concentrations in the collected supernatants were measured by ELISA (see VEGF analysis by ELISA). The bioactivity of released VEGF was determined by endothelial cell proliferation assay. Details of these procedures are given in section Bioactivity of VEGF.

To obtain more information on the mechanism of release, the release data obtained from SE-UPLC and ELISA analysis were fitted using the Korsmeyer-Peppas model, where $Q_{t}$ is the amount of drug released from microspheres at time point $t, Q_{0}$ is the initial amount of drug in microspheres, $n$ is diffusional exponent indicative of the transport mechanism, and $K_{p}$ is Korsmeyer-Peppas constant incorporating structural and geometric characteristics of the dosage form (equation 5)..$^{54}$ The diffusional exponent $n$ was calculated from the fitted linear regression lines of log (\% drug released) versus log (time) (equation 6).

$$
\frac{Q_{t}}{Q_{0}}=K_{p} \times t^{n}
$$

EQUATION 5: Korsmeyer-Peppas equation.

$$
\log \left(\frac{Q_{t}}{Q_{0}}\right)=\log \left(K_{p}\right)+n \log (t)
$$

EQUATION 6: adapted Korsmeyer-Peppas equation.

\section{VEGF analysis by SE-UPLC}

Samples were analyzed without dilution using size exclusion ultra-performance liquid chromatography (SE-UPLC) on a Waters Acquity H-class UPLC system (Waters, Milford, USA) with FLR-detector, operated at $\lambda_{\text {ex }}: 276 \mathrm{~nm}, \lambda_{\mathrm{em}}: 310 \mathrm{~nm}$. The system was equipped with a Waters Acquity UPLC Protein BEH SEC column (150 mm x $4.6 \mathrm{~mm}$; $1.7 \mu \mathrm{m})$ maintained at $25^{\circ} \mathrm{C}$. The mobile phase consisted of a $50 \mathrm{mM}$ phosphate, $0.4 \mathrm{M}$ perchlorate buffer $\mathrm{pH} 6.3$ : acetonitrile ( $90: 10, v / v)$, and was operated at a flow rate of $0.30 \mathrm{ml} / \mathrm{min}$. Sample aliquots of $10 \mu \mathrm{l}$ were automatically injected. The VEGF retention time under these condition was $4.5 \mathrm{~min}$. The linear range of the VEGF calibration curve was $2 \mu \mathrm{g} / \mathrm{ml}$ (detection limit) - $200 \mu \mathrm{g} / \mathrm{ml} \mathrm{VEGF.}$

\section{VEGF analysis by ELISA}

In vitro release samples were analyzed by a sandwich enzyme-linked immunosorbent assay (ELISA) (Human VEGF DuoSet ELISA, R\&D Systems, Abingdon, United Kingdom) according to the manufacturer's protocol. The VEGF stock solution of the ELISA kit was used for calibration in the concentration range of $31-2000 \mathrm{pg} / \mathrm{ml}$. Release samples were diluted with reagent 
diluent to fall within the working range of the assay and measured in duplicate. ELISA plates were read at $450 \mathrm{~nm}$ using a Spectrostar Nano plate reader (BMG Labtech, Ortenberg, Germany).

\section{Comparison VEGF detection by ELISA and SE-UPLC}

VEGF detection by ELISA and SE-UPLC was compared by quantifying fresh, non-formulated VEGF in solution with defined concentrations. A $1 \mathrm{mg} / \mathrm{ml}$ VEGF solution in IVR buffer was prepared by reconstituting $1 \mathrm{mg}$ of freeze-dried VEGF, as received by Peprotech, in $1 \mathrm{ml}$ of IVR buffer (Dulbecco's PBS pH 7.4 (0.2 $\mu \mathrm{m}$ filtered), 0.025\% Tween 20, 0.02\% $\mathrm{NaN}_{3^{\prime}}$, see In vitro release studies). This solution was diluted further in IVR buffer to VEGF solutions of 6 , 60, and $160 \mu \mathrm{g} / \mathrm{ml}$. VEGF was quantified by ELISA and SE-UPLC. Similarly, IVR samples from microspheres (as described in in vitro release studies) were spiked with $50 \mu \mathrm{g} / \mathrm{ml}$ of fresh, nonformulated VEGF in IVR buffer. The samples were measured by SE-UPLC and ELISA.

\section{Storage stability: Reproducibility of release profiles}

\section{Stability after repeated freezing and storage at $-20^{\circ} \mathrm{C}$}

To study the effect of repeated storage at $-20^{\circ} \mathrm{C}$ on the in vitro release profile, $90 \mathrm{mg}$ of freshly prepared freeze-dried microspheres (as described in Preparation of monodisperse microspheres, $1.0 \mathrm{wt} \%$ target VEGF loading) were accurately weighed, transferred into a $1.5 \mathrm{ml}$ Eppendorf tube and stored at $-20^{\circ} \mathrm{C}$ for a minimum of 16 hours. Next, the microsphere sample was taken out of the freezer and incubated at room temperature for at least 3 hours. A $10 \mathrm{mg}$ microsphere sample was removed and subjected to an in vitro release study, as described in sections In vitro release studies and VEGF analysis by SE-UPLC (release measured by SE-UPLC). The remaining microsphere sample was placed back into the $-20^{\circ} \mathrm{C}$ freezer. This procedure was repeated five times. VEGF release profiles, obtained after repeated freezing to and storage at $-20^{\circ} \mathrm{C}$, were compared to the release profiles from freshly prepared microspheres $(t=0)$.

\section{Long-term storage stability at $-20^{\circ} \mathrm{C}$}

The stability of VEGF-loaded microspheres was assessed for storage durations of 1, 2, 3, 6 and 9 months at $-20^{\circ} \mathrm{C}$. In detail, $90 \mathrm{mg}$ of freeze-dried VEGF-loaded microsphere samples (prepared as described in section Preparation of monodisperse microspheres, $1.0 \mathrm{wt} \%$ target VEGF loading) were accurately weighed, transferred into a $1.5 \mathrm{ml}$ Eppendorf tube and stored at $-20{ }^{\circ} \mathrm{C}$. After the indicated storage period, the samples were removed from the freezer and were incubated at room temperature for at least 3 hours. Afterwards, the microsphere samples were subjected to an in vitro release study, as described in sections In vitro release studies and VEGF analysis by SE-UPLC (release measured by SE-UPLC). The release profiles (obtained after various storage duration at $-20^{\circ} \mathrm{C}$ ) were compared to the release profile of freshly prepared freeze-dried microspheres $(t=0)$. 


\section{Similarity factor $f_{2}$}

The SE-UPLC release curves of microspheres after storage at $-20^{\circ} \mathrm{C}$ (sections Stability after repeated freezing and storage at $-20{ }^{\circ} \mathrm{C}$ and Long-term storage stability at $-20{ }^{\circ} \mathrm{C}$ ) were compared by calculation of the similarity factor $f_{2}$. The differences of amount of drug released from a reference batch $(R)$ and from a test batch $(T)$ at time point $t$ are further transformed according to equation 7. Two release curves are regarded similar when $f_{2}$ is between 50 and 100 , whereby a $f_{2}$ value of 50 represents a $10 \%$ difference and 100 represents fully equal release curves. Two release curves are regarded different if $f_{2}<50.55,56$

$$
\begin{array}{r}
f_{2}=50 \times \log \left\{\left[1+\left(\frac{1}{n}\right) \sum_{t=1}^{n}\left(R_{t}-T_{t}\right)^{2}\right]^{-0.5} \times 100\right\} \\
\text { EQUATION 7: Similarity factor } f_{2}
\end{array}
$$

\section{Bioactivity of VEGF}

The bioactivity of released VEGF was determined using an in-house developed Alamar Blue proliferation assay with human umbilical vein endothelial cells (HUVEC) ${ }^{57-59}$. HUVECs (Lonza, Switzerland) were cultured until passage 2 to 5 in EBM-2 complete medium (Lonza CC-3162) supplemented with growth factor bullet kit. Actual proliferation assays were performed in a growth-factor poor medium (bioactivity medium). Addition of VEGF to the medium was able to enhance proliferation approximately 3.5-fold. VEGF standards (10 - $100 \mathrm{ng} / \mathrm{ml}$ ) were prepared in M199 medium supplemented with 2.5\% FBS (bioactivity medium). Release samples were collected at different time points $(1,7,14,21,28$ days) and diluted 500 times in order to fall within the (linear) proliferation range for non-formulated VEGF $(10-100 \mathrm{ng} / \mathrm{ml})$. Prior to the experiment, 96-well plates (Greiner Bio-One, the Netherlands) were coated overnight at 4 'C with $100 \mu$ l of coating solution consisting of $50 \mu \mathrm{g} / \mathrm{ml}$ collagen I, rat tail (Corning 354236, USA) in $20 \mathrm{mM}$ acidic acid. On the day of the experiment, plates were washed twice with PBS before seeding of the cells. Subsequently, the wells were filled with $100 \mu$ l of sample or VEGF standard and 4000 cells suspended in $100 \mu$ l of bioactivity medium (making a total of $200 \mu \mathrm{l}$ per well). Wells without cells and filled with $200 \mu$ l of bioactivity medium served as controls. Cells were incubated at $37{ }^{\circ} \mathrm{C} / 5 \% \mathrm{CO}_{2}$ for 92 hours, after which $20 \mu \mathrm{l}$ of Alamar Blue reagent was added to the wells. The plates were incubated for another 4 hours, before measuring the fluorescence $\left(\lambda_{\text {ex }} 530 \mathrm{~nm}\right.$ and $\lambda_{\text {em }} 600 \mathrm{~nm}$ ) with a microplate reader (Berthold Mithras LB 940, Germany). The results are expressed as relative cell proliferation, which is the proliferation in $\%$ normalized by the proliferation of cells that were incubated without VEGF.

\section{Statistical analysis}

Data are presented as average with standard deviation. Statistical analysis was performed with Graphpad Prism7 using one-way analysis of variance (ANOVA) and Tukeys multicomparison test. Differences between analyzed groups were considered significant if $p<0.05$. 


\section{Results and Discussion}

\section{Selection of suitable blend composition}

Microsphere batches were prepared with a target VEGF loading of 0.2 wt $\%$ and different blend ratios of multiblock copolymers $A$ and $B$ in order to obtain a microsphere formulation with the desired release profile, i.e. a minimal burst followed by a sustained release for 4 weeks. These polymers were selected based on previous work of Teekamp et al. who achieved a 2-week controlled release profile of human serum albumin (HSA) from microspheres based on multiblock copolymers $A$ and $B .{ }^{48}$ The inner water phase of the emulsified droplets consisted of $5 \mathrm{mM}$ succinate buffer $\mathrm{pH}$, which is similar to the buffer used by Cleland et al. for the preparation of solid VEGF microparticles by spray-drying, which were subsequently encapsulated into PLGA microparticles by solid-in-oil emulsification. ${ }^{19}$ Microspheres were prepared by double emulsion method in which a water-in-oil $\left(w_{1} / 0\right)$ primary emulsion was processed over a stainless steel membrane with $20 \mu \mathrm{m}$ pores. The obtained microspheres were collected after extraction and evaporation of the organic solvent, washed and without further sieving or fractionating freeze-dried. The freeze-dried microspheres consisted of uniformly sized microparticles with average diameters ranging between 42 and $51 \mu \mathrm{m}$ and narrow size distribution (12 - 28\% CV, table S2), characteristic for membrane emulsification. ${ }^{46}$ The diameter of the obtained particles is approximately twice that of the pores in the membrane, which is a common observation with membrane emulsification. At high dispersed phase flow rates, extra dispersed phase is pressed into the formed droplet with an initial size equal to the pore diameter before it detaches, resulting in a larger droplet diameter. ${ }^{46}$

SEM analysis (figure S2) showed differences in morphology of the microspheres depending on the polymer blend composition. Microspheres prepared with polymer A or a blend of polymer A and B were porous whereas microspheres prepared with polymer B only had a smooth surface with scattered visible pores.

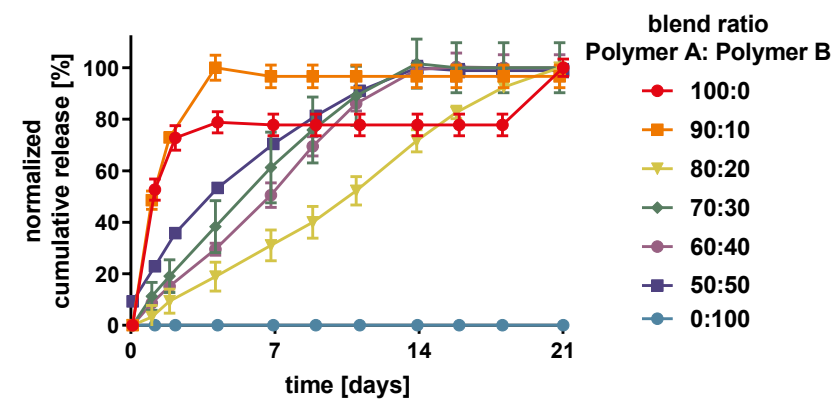

FIGURE 2: Cumulative release of VEGF from microspheres based on various blends of multiblock polymer $A$ and $B$ (shown in legend) and target VEGF loading of $0.2 \mathrm{wt} \%$. The release was performed at $37^{\circ} \mathrm{C}$ in $\mathrm{PBS} \mathrm{pH} 7.4$ supplemented with $0.025 \%$ Tween 20 and $0.02 \% \mathrm{NaN}_{3}$. Released VEGF was quantified by SE-UPLC. 
Microspheres were screened based on their 3-week release pattern, as the growth factor release pattern in this time period is crucial for vascularization of biomaterial implants. ${ }^{13}$ VEGF release was measured by SE-UPLC and is plotted as normalized cumulative VEGF. Total cumulative release after 21 days is assumed to represent complete release of encapsulated VEGF (with the exception of 0:100 blend ratio).

Microspheres based on a 90:10 blend of polymer A and B and of 100\% polymer A showed high initial VEGF release during the first 2 days followed by a period of 19 days without any further release. As we aimed for a formulation demonstrating continuous release for several weeks, these formulations were not suitable for our purposes. In contrast, microspheres based on polymer B only did not release VEGF during the three-week timespan of the release experiment. Possibly, these microspheres had a low VEGF loading efficiency and therefore no VEGF release was observed. This observation is in line with the release study published by Teekamp et al. who also observed no release of HSA in 2 weeks from microspheres prepared with polymer B. ${ }^{48}$ This microsphere formulation also did not meet our release profile target. Lastly, the release profiles of VEGF from microspheres based on various blends of polymer $A$ and B (i.e. 80:20, 70:30, 60:40, 50:50) showed continuous release for around 2 weeks with minimal burst release. However, the differences between release profiles from microspheres based on 80:20, 70:30 and 60:40 blends were minimal. The average release rate of these blends was approximately 5-7\% release per day for a continuous period of at least 14 days, which met our initial selection criteria for release kinetics. Slight differences in sustained release pattern are likely an interplay between total PEG content and PEG chain lengths of each polymer. Previous studies with similar multiblock copolymers have shown that the release of proteins from these polymers is governed by the PEG content which leads to swelling of the microspheres, and subsequent diffusion of protein through water-filled pores. ${ }^{41-43}$

For further development, the 50:50 blend of polymer A and B was selected due its preferred release kinetics, i.e. continuous release without significant burst release, which was also the blend that had been selected by Teekamp et al. for delivery of pPB-HSA. ${ }^{48}$

\section{In vitro degradation study of microspheres prepared with 50:50 blend ratio}

The degradation properties of microspheres based on a 50:50 blend of polymer $A$ and $B$ was studied with non-loaded placebo microspheres. These microspheres had a porous morphology (figure $3 a$, left image " $0 \mathrm{~d}$ ") and a narrow size distribution ( $40 \pm 6 \mu \mathrm{m}$ ) comparable to VEGF-loaded microspheres prepared with the same polymer blend (table S2 and figure S2). During incubation in IVR buffer for 28 days, the microspheres retained their spherical shape and porous morphology, as shown in figure $3 a$, indicating that hardly any erosion took place. This observation is in line with the degradation data as shown in figure $3 b$, which shows that after a slight decrease in mass of $20 \%$ after 7 days, the remaining dry mass did not change the next 21 days. The composition of degrading microspheres over time was analyzed for PEG 
content. Figure 3c shows that the PEG content decreased by $30 \%$ within 7 days, and then no further changes occurred up to 28 days. GPC analysis of degradation samples showed that the molecular weight of the polymers was reduced significantly after 7 days of incubation (figure S3). From 7 days to 28 days, a further slow reduction in molecular weights of the polymers was observed. These results indicate that in the first 7 days, a small fraction of PEG was cleaved resulting in chain scissions in the polymers and diffusion of PEG out of the polymer matrix. This observation is in agreement with previous studies reporting that PEG is shed specifically during early stages of degradation due to preferential cleavage of the ester bonds that connect PEG and PCL units. ${ }^{60-63}$ Analysis by ${ }^{1} \mathrm{H}-\mathrm{NMR}$ before and after addition of shift reagent TAIC showed that the constitution of the polymers remained similar during degradation up to 28 days (figures S4 and S5). The almost constant weight of the degrading samples and their stable composition between 7 and 28 days demonstrate that hydrolysis of ester bonds in the PCL and PLLA blocks hardly occurred. All in all, our results show that the slight initial loss in mass after 7 days of incubation is likely due to hydrolysis of PEG-PCL bonds, resulting in loss of a small fraction of PEG and causing a decrease in molecular weight of the degrading polymers. Afterwards, molar composition of the polymers remained unchanged until the end of the degradation study. Therefore, no extensive degradation occurred during 28 days of incubation.

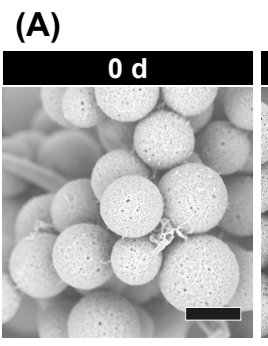

(B)

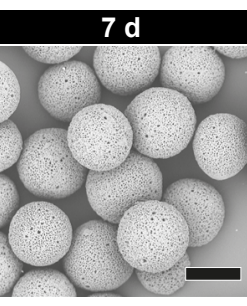

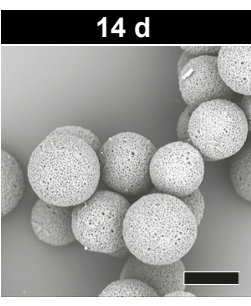

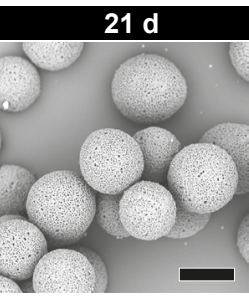

(C)
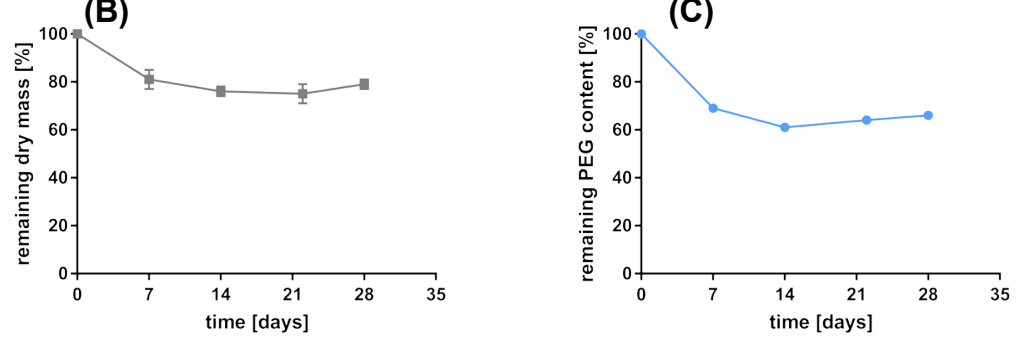

FIGURE 3: In vitro degradation of placebo microspheres prepared with polymer A: polymer B blend of 50:50 after incubation at $37{ }^{\circ} \mathrm{C}$ in PBS pH 7.4, supplemented with $0.025 \%$ Tween 20 and $0.02 \% \mathrm{NaN}_{3}$. A: SEM images before and after incubation. Scale bars represents $30 \mu \mathrm{m}$. The time of incubation ( $\mathrm{d}$ : days) is stated above each image, whereby "0 d" shows freshly prepared microspheres before incubation. B: Remaining microsphere dry mass after 28 days, C: remaining PEG content in the microsphere samples, as determined by ${ }^{1} \mathrm{H}-\mathrm{NMR}$. 


\section{Optimized microsphere formulation based on 50:50 polymer blend}

For further studies, three microsphere batches with a higher target loading (1.0 wt\%) were prepared with a 50:50 blend of polymer A and B. The preparation method was slightly modified by increasing batch size and VEGF/polymer ratio (table 3, experimental section). These modifications aimed at increasing VEGF loading content and loading efficiency (as discussed in section Selection of suitable blend composition). The loading content of VEGF of microspheres prepared with a 0.2 wt $\%$ target loading could not be accurately analyzed, as VEGF concentrations in the samples were below the detection limit of the developed assay. Possible modifications such as increasing the amount of microspheres used for the protocol were not feasible, as it would have required dissolving 380 mg microspheres in $0.5 \mathrm{ml}$ DMSO in order to fall within the calibration curve of the assay (assuming a loading efficiency of 20\%).

Microspheres prepared with a $1.0 \mathrm{wt} \%$ target loading had a narrow size distribution (table 3, average sizes between 44 and $52 \mu \mathrm{m}, \mathrm{CV}$ between 15 and $30 \%$, size distribution plots shown in figure S6). SEM analysis of the obtained microspheres showed a smooth surface with small pores (figure 4). Table 1 summarizes the characteristics of the obtained microspheres, per microsphere batch and average values for the three individually prepared batches. The batch yields ranged from 65 to $74 \%$. VEGF loading was determined by BCA protein assay after destruction of the particles and corresponded to a high average loading efficiency of $78 \pm 16 \%$. The increased encapsulation efficiency as compared to microspheres with a target loading of $0.2 \mathrm{wt} \%$ (as described in section Selection of suitable blend composition) can be explained by the introduced modifications, in line with previous experiments. ${ }^{64-67}$ An increased polymer concentration of the oil phase leads to a shorter time of solidification as less solvent has to be removed. This in turn decreases the diffusion of the protein into the continuous phase and thereby increases the loading efficiency. ${ }^{64} \mathrm{All}$ in all, three microsphere batches were prepared with similar characteristics regarding yield, VEGF loading and loading efficiency, thereby demonstrating low batch-to-batch variation and good batch reproducibility.

TABLE 3: Formulation characteristics of three batches of VEGF-loaded microspheres prepared with a 50:50 blend ratio of polymer $A$ and $B$, per batch and average values \pm standard deviation (SD) for each formulation characteristic.

\begin{tabular}{cccccc}
\hline batch & yield [\%] & average size [ $\boldsymbol{\mu m}$ ]; CV [\%] & $\begin{array}{c}\text { feed loading } \\
\text { [wt\%] }\end{array}$ & $\begin{array}{c}\text { actual loading } \\
\text { [wt\%] }\end{array}$ & $\begin{array}{c}\text { loading } \\
\text { efficiency [\%] }\end{array}$ \\
\hline 1 & 74 & $51 ; 15$ & 1.00 & 0.72 & 72 \\
2 & 73 & $52 ; 30$ & 0.93 & 0.90 & 96 \\
3 & 65 & $44 ; 17$ & 1.13 & 0.76 & 67 \\
\hline average + SD & $\mathbf{7 1 \pm \mathbf { 5 }}$ & $\mathbf{4 9 \pm \mathbf { 4 }}$ & $\mathbf{1 . 0 2} \pm \mathbf{0 . 1 0}$ & $\mathbf{0 . 7 9} \pm \mathbf{0 . 0 9}$ & $\mathbf{7 8 \pm 1 6}$ \\
\hline
\end{tabular}

aLoading of VEGF in microspheres was determined after destruction of loaded microspheres in DMSO and $\mathrm{NaOH}$. VEGF content was quantified by BCA protein assay. 

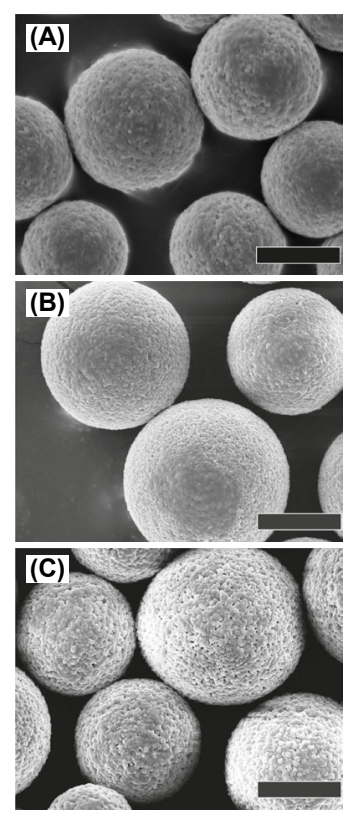

FIGURE 4: Morphology of VEGF-loaded microspheres (three individually prepared batches) prepared with a 50:50 blend of polymer A and B and a target loading of 1.0 wt\% (see table 1). SEM images; A: batch 1, B: batch 2, C: batch 3. Scale bar represents $20 \mu \mathrm{m}$.

\section{In vitro VEGF release}

VEGF release from obtained microspheres was measured by SE-UPLC and ELISA, as shown in figure 5. Both cumulative release profiles were normalized based on the cumulative release at the end of the release study (day 30 for ELISA data and day 25 for SE-UPLC data). Microspheres of the 50:50 polymer blend released VEGF continuously over a period of $\sim$ four weeks with a low burst release. Normalized release profiles for both methods are in good agreement (figure 5, blue and red circles). These release curves were fitted using the Korsmeyer-Peppas model (figure 5, blue and red dotted lines). ${ }^{54,55}$ The calculated parameters, i.e. diffusional exponent, $95 \%$ confidence interval and correlation coefficient, of this fit are shown in table 2. Both ELISA and SE-UPLC release data show a good fit for this model ( $R^{2}: 0.97$ (SE-UPLC data) and 0.96 (ELISA data)). The diffusional exponents $n$ were $0.41 \pm 0.02$ for SE-UPLC release data and 0.42 \pm 0.02 for ELISA release data, suggesting diffusion-related release. Taking the release profiles and the results of the degradation study (section In vitro degradation study of microspheres prepared with 50:50 blend ratio) together, it can be concluded that VEGF release from microspheres occurs via diffusion of VEGF through a water-filled porous network, caused by the hydrophilicity of PEG within the multiblock copolymer, as no extensive polymer erosion occurs. 


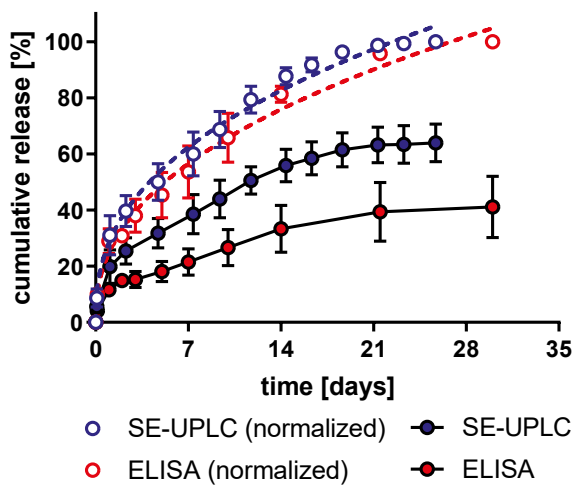

FIGURE 5: Cumulative release of VEGF from microspheres based on a 50:50 blend and a target VEGF loading of 1.0 wt $\%$. The release was performed at $37^{\circ} \mathrm{C}$ in PBS pH 7.4 supplemented with $0.025 \%$ Tween 20 and $0.02 \% \mathrm{NaN}_{3}$. Released VEGF was measured by ELISA (red) and SE-UPLC (blue), and plotted as cumulative release (filled blue and red circles, connected by a black line) and as normalized cumulative release (blue and red circles). Normalized cumulative release data were fitted using the Korsmeyer-Peppas model (blue and red dotted lines). Parameters of this fit are listed in table 4.

TABLE 4: Korsmeyer-Peppas model fit parameters for normalized cumulative release curves of SE-UPLC and ELISA data, as shown in figure 5 as blue and red dotted lines, respectively.

\begin{tabular}{ccc}
\hline \multirow{2}{*}{ parameters } & \multicolumn{2}{c}{ normalized cumulative release } \\
\cline { 2 - 3 } & SE-UPLC & ELISA \\
\hline$n$ & $0.41 \pm 0.02$ & $0.42 \pm 0.02$ \\
$95 \% \mathrm{Cl}$ & $0.37-0.44$ & $0.39-0.46$ \\
$R^{2}$ & 0.97 & 0.96 \\
\hline
\end{tabular}

$n$ : diffusional exponent, $95 \% \mathrm{Cl}: 95 \%$ confidence interval, $R^{2}$ : correlation coefficient.

Based on loading determination with BCA assay, VEGF was released up to 60\% based on SEUPLC data (figure 5). In contrast, cumulative VEGF release by ELISA was 40\% (figure 5), most likely due to the numerous dilution steps towards the working range of the ELISA kit (31.3 - 2000 $\mathrm{pg} / \mathrm{ml}$ ) which might have resulted in protein loss due to aspecific adsorption onto materials the protein is exposed to during sample handling. Similar underestimation of the VEGF levels were observed with freshly prepared VEGF solutions in IVR buffer and with IVR samples spiked with a defined VEGF concentration (tables S3 and S4). In previous publications on VEGF-loaded PLGA-based microspheres, VEGF release quantified by ELISA reached cumulative release of $>$ $50 \%$ after four weeks. ${ }^{28,29,68}$ Since the formulations of the mentioned studies were prepared at much lower VEGF target loading (up to 100-fold lower as compared to our target loading of $1.0 \mathrm{wt} \%)$, those studies did not require extensive dilution for ELISA purposes. Nevertheless, the observed release profiles of our microspheres determined either by SE-UPLC or ELISA correlate well, corroborating the integrity of the released VEGF. To our knowledge, our study is unique in comparing two analytical methods to measure protein release from microspheres. 
The VEGF release profile of microspheres based on a 50:50 blend of polymer A and B (figure 5) differs substantially from published release curves of VEGF from PLGA microparticles. Several groups have reported biphasic release profiles with a high burst release (around $60 \%$ of the loading), followed by sustained release of VEGF for 4 weeks accumulating to $70-75 \%$ of the loaded amount. 28,29 Similarly, sustained release of VEGF from PLGA microspheres of $~ 60 \%$ up to 7 days has been reported, followed by slow VEGF release reaching $70 \%$ after 28 days. ${ }^{68}$ In contrast to this, the VEGF-loaded microspheres used in this study, which are based on the swellable multiblock copolymers A and B in a ratio of 50:50, showed a low burst release and sustained release for nearly four weeks reaching 60\% (as measured by SE-UPLC). The difference between release profiles of PLGA-based and [PCL-PEG-PCL]-b-[PLLA]-based microspheres can be explained by the surface-bound protein and protein close to the surface of PLGAbased microspheres that is released nearly completely within the first day "burst", before the onset of erosion. In contrast, microspheres based on [PCL-PEG-PCL[- $b$-[PLLA] polymers release their encapsulated cargo via diffusion through a water-filled polymer matrix, caused by the hydrophilic PEG blocks.

\section{Storage stability at $-20^{\circ} \mathrm{C}$}

An important aspect of developing microspheres is preventing protein degradation and aggregation during preparation, storage and release. ${ }^{23,24,69}$ It is essential that the release pattern of VEGF from freshly prepared microspheres and after long-term storage remains unchanged. Although previous studies have shown that the addition of cryoprotectants, such as trehalose, increases the stability of proteins formulated in PLGA nanoparticles after freezedrying, the repeated storage and long-term storage at $-20^{\circ} \mathrm{C}$ has not been investigated. ${ }^{70,71}$ In contrast, the freeze-thaw stability and long-term storage of aqueous protein formulations is well studied, showing that the frozen storage of aqueous protein formulations can cause degradation and aggregation of proteins. ${ }^{72,73}$ We therefore investigated the storage stability at $-20{ }^{\circ} \mathrm{C}$ of VEGF-loaded microspheres by comparing VEGF release profiles from freshly prepared microspheres with VEGF release profiles from microspheres exposed to repeated freezing and storage at $-20^{\circ} \mathrm{C}$ as well as long-term storage at $-20^{\circ} \mathrm{C}$.

Freeze-dried VEGF-loaded microspheres prepared with 50:50 blend of polymer A and B, as characterized in figure 4 and 5 and table 3 and 4 , were frozen and stored at $-20^{\circ} \mathrm{C}$ overnight and subsequently incubated at room temperature for 3 hours multiple times. After incubation at room temperature, a microsphere sample was removed for analyzing VEGF release with SE-UPLC. The remaining microspheres were returned to $-20^{\circ} \mathrm{C}$ storage. As shown in figure S8a, release profiles of VEGF from microspheres repeatedly frozen (up to 5 times) remained highly similar to the release profile of freshly prepared microspheres, which was confirmed by similarity index $f_{2}$ values of $>70$ (table $\left.S 5\right) .^{56}$ 
Microspheres were also stored at $-20^{\circ} \mathrm{C}$ for 1, 2, 3, 6 and 9 months. At these time periods, microspheres were incubated at room temperature for at least 3 hours, dispersed in IVR buffer and incubated at $37^{\circ} \mathrm{C}$. Released VEGF was quantified by SE-UPLC. VEGF release profiles from freshly prepared microspheres and from microspheres stored at $-20{ }^{\circ} \mathrm{C}$ up to 9 months are shown in figure S8b. No distinct differences between release profiles can be seen, confirmed by similarity factor $f_{2}$ of $>50$ (table S6). ${ }^{56}$ Neither repeated storage at $-20{ }^{\circ} \mathrm{C}$ nor long-term $-20{ }^{\circ} \mathrm{C}$ storage of microspheres altered the release profile of VEGF from microspheres, as compared to release profiles from freshly prepared microspheres. Therefore, microspheres based on a 50:50 blend ratio of polymer A and B and loaded with VEGF present excellent storage stability.

\section{Bioactivity of released VEGF}

It is crucial that released VEGF from the microspheres over the 4 weeks period retained its bioactivity as this is necessary for stable blood vessel formation ${ }^{31}$. Figure 6 shows the bioactivity of released VEGF as compared to the native non-formulated VEGF, expressed as relative cell proliferation. The released protein at different time points led to relative cell proliferation of around 3. All released VEGF samples fall within the responsive range of the assay (1 - 3.5 relative cell proliferation). Supernatants of placebo microspheres served as controls. These control samples, containing possible degradation products of placebo microspheres such as PEG (as discussed above with figure 3), did not induce proliferation.

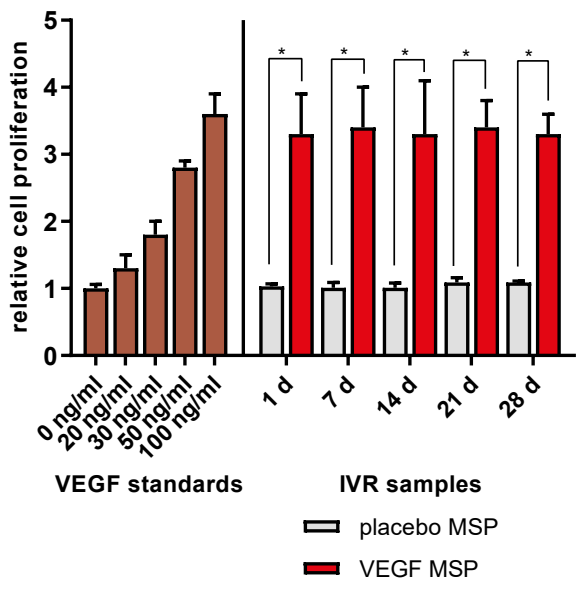

FIGURE 6: Bioactivity of released VEGF expressed as relative cell proliferation. On the left side of the graph (dark red bars): responsive range of VEGF standards between 0 and $100 \mathrm{ng} / \mathrm{ml}$ ( $\mathrm{n}=3$ per concentration). On the right side of the graph (red and gray bars): relative cell proliferation of endothelial cells incubated with IVR supernatants collected from VEGF-loaded microspheres ("VEGF MSP", red bars) or placebo microspheres ( "placebo MSP", gray bars). Microspheres had been stored at $-20^{\circ} \mathrm{C}$ for several months and have been exposed to several freezing cycles prior to the bioactivity assay. Supernatants of each time point were diluted in culture medium and incubated in triplicate wells (bars represent average \pm standard deviation of $n=3$ ). * $p<0.05$ VEGF MSP versus placebo MSP. 
The IVR buffer used for these cell-based bioactivity experiments was supplemented with BSA and antibiotics (bioactivity IVR buffer), instead of Tween 20 and sodium azide (components of IVR buffer for release studies in figures 2 and 5), as these materials inhibited cell proliferation (data not shown). As a consequence, the VEGF concentration in the release supernatants could not be determined by SE-UPLC, as BSA interfered with the VEGF peak of the SE-UPLC chromatogram. The concentrations were therefore estimated with ELISA and normalized based on the cumulative release by SE-UPLC as presented in section In vitro release VEGF). ELISA release data had a good Korsmeyer-Peppas model fit ( $\left.R^{2}: 0.98\right)$ and a diffusional exponent of $0.36 \pm 0.05$ (95\% confidence interval: 0.22 - 0.55), comparable to ELISA release data in standard IVR buffer (as discussed in section In vitro release VEGF, figure S9). Our results demonstrate that bioactivity of released VEGF was fully retained within the experimental error of the bioactivity assay. In several previous studies, the bioactivity of released VEGF was determined by similar HUVEC viability-based assays, however, these studies reported the bioactivity of VEGF released in the beginning of the release period only, i.e. up to 1 week. ${ }^{68,74}$ In contrast, we assessed the bioactivity of VEGF that is released throughout the entire release period, including between week 2, 3 and 4 of release. Precisely the later period of release, i.e. between 2 and 4 weeks, is critical for stable blood vessel formation. ${ }^{14,31}$ The bioactivity assay has been performed with VEGF microspheres that have been stored for several months storage at $-20^{\circ} \mathrm{C}$ and subjected to several freezing cycles. We therefore conclude that VEGF formulated in the microspheres remains bioactive for prolonged time.

Based on the data shown in this study, we estimate that a dose of 1 mg VEGF microspheres will provide an average release rate of $150 \mathrm{ng}$ VEGF/day for 4 weeks, which has been shown to be sufficient for vascularization of a $0.1 \mathrm{ml}$ biomaterial implant. ${ }^{14}$

\section{Conclusions}

In this study, we have developed monodisperse VEGF-loaded microspheres that exhibit sustained release kinetics during four weeks. This release profile is considered optimal for successful vascularization of a tissue-engineered construct. The developed VEGF-loaded microspheres were prepared with blends of [PCL-PEG-PCL]-b-[PLLA] multiblock copolymers with various PEG molecular weight and content, using a solvent extraction-based membrane emulsification process. The microspheres had a VEGF loading of 0.79 wt $\%$ and loading efficiency of 78\%. VEGF release is governed by diffusion through the water-swollen polymer matrix. Released VEGF remained fully bioactive over the entire release period of four weeks. Therefore, microspheres based on multiblock copolymers and loaded with VEGF are an attractive vascularization platform for incorporation into tissue engineered constructs. 


\section{Acknowledgements}

This research is funded by European Union's Horizon 2020 research and innovation program under grant agreement number 645991 (DRIVE). The authors thank Christine Hiemstra, Prachi Desai and Sjaak Jong for their contribution to this work. Yan Wang is greatly acknowledged for the GPC analysis, as well as Mies van Steenbergen for ${ }^{1} \mathrm{H}-\mathrm{NMR}$ analysis. 


\section{References}

1. Langer, R.; Vacanti, J. P. Tissue engineering. Science 1993, 260, (5110), 920-6.

2. Khademhosseini, A.; Langer, R. A decade of progress in tissue engineering. Nat. Protoc. 2016, 11, (10), 1775-81.

3. Berthiaume, F.; Maguire, T. J.; Yarmush, M. L. Tissue engineering and regenerative medicine: history, progress, and challenges. Annu Rev Chem Biomol Eng 2011, 2, 403-30.

4. Crapo, P. M.; Gilbert, T. W.; Badylak, S. F. An overview of tissue and whole organ decellularization processes. Biomaterials 2011, 32, (12), 3233-43.

5. Stratton, S.; Shelke, N. B.; Hoshino, K.; Rudraiah, S.; Kumbar, S. G. Bioactive polymeric scaffolds for tissue engineering. Bioact Mater 2016, 1, (2), 93-108.

6. Ovsianikov, A.; Khademhosseini, A.; Mironov, V. The synergy of scaffold-based and scaffold-free tissue tngineering strategies. Trends Biotechnol. 2018, 36, (4), 348-357.

7. Llames, S.; Garcia, E.; Otero Hernandez, J.; Meana, A. Tissue bioengineering and artificial organs. Adv. Exp. Med. Biol. 2012, 741, 314-36.

8. Novosel, E. C.; Kleinhans, C.; Kluger, P. J. Vascularization is the key challenge in tissue engineering. Adv Drug Deliv Rev 2011, 63, (4-5), 300-11.

9. Fu, J.; Wang, D. A. In situ organ-specific vascularization in tissue engineering. Trends Biotechnol. 2018, 36, (8), 834-849.

10. Rouwkema, J.; Rivron, N. C.; van Blitterswijk, C. A. Vascularization in tissue engineering. Trends Biotechnol. 2008, 26, (8), 434-41.

11. Yla-Herttuala, S.; Rissanen, T. T.; Vajanto, I.; Hartikainen, J. Vascular endothelial growth factors: biology and current status of clinical applications in cardiovascular medicine. J. Am. Coll. Cardiol. 2007, 49, (10), 1015-26.

12. Cao, L.; Mooney, D. J. Spatiotemporal control over growth factor signaling for therapeutic neovascularization. Adv Drug Deliv Rev 2007, 59, (13), 1340-50.

13. Silva, E. A.; Mooney, D. J. Effects of VEGF temporal and spatial presentation on angiogenesis. Biomaterials 2010, $31,(6), 1235-41$.

14. Davies, N.; Dobner, S.; Bezuidenhout, D.; Schmidt, C.; Beck, M.; Zisch, A. H.; Zilla, P. The dosage dependence of VEGF stimulation on scaffold neovascularisation. Biomaterials 2008, 29, (26), 3531-8.

15. Said, S. S.; Pickering, J. G.; Mequanint, K. Advances in growth factor delivery for therapeutic angiogenesis. J. Vasc. Res. 2013, 50, (1), 35-51.

16. Lee, K.; Silva, E. A.; Mooney, D. J. Growth factor delivery-based tissue engineering: general approaches and a review of recent developments. J R Soc Interface 2011, 8, (55), 153-70.

17. Phelps, E. A.; Templeman, K. L.; Thule, P. M.; Garcia, A. J. Engineered VEGF-releasing PEG-MAL hydrogel for pancreatic islet vascularization. Drug Deliv Transl Res 2015, 5, (2), 125-36.

18. Babasola, I. O.; Rooney, M.; Amsden, B. G. Corelease of bioactive VEGF and HGF from viscous liquid poly(5ethylene ketal epsilon-caprolactone-co-D,L-lactide). Mol. Pharm. 2013, 10, (12), 4552-9.

19. Cleland, J. L.; Duenas, E. T.; Park, A.; Daugherty, A.; Kahn, J.; Kowalski, J; Cuthbertson, A. Development of poly( $D, L-$ lactide--coglycolide) microsphere formulations containing recombinant human vascular endothelial growth factor to promote local angiogenesis. J. Control. Release 2001, 72, (1-3), 13-24.

20. Freiberg, S.; Zhu, X. X. Polymer microspheres for controlled drug release. Int. J. Pharm. 2004, 282, (1-2), 1-18.

21. Ma, G. Microencapsulation of protein drugs for drug delivery: strategy, preparation, and applications. J. Control. Release 2014, 193, 324-40.

22. Prajapati, V. D.; Jani, G. K.; Kapadia, J. R. Current knowledge on biodegradable microspheres in drug delivery. Expert Opin Drug Deliv 2015, 12, (8), 1283-99.

23. van de Weert, M.; Hennink, W. E.; Jiskoot, W. Protein instability in poly(lactic-co-glycolic acid) microparticles. Pharm. Res. 2000, 17, (10), 1159-67.

24. Schwendeman, S. P. Recent advances in the stabilization of proteins encapsulated in injectable PLGA delivery systems. Crit. Rev. Ther. Drug Carrier Syst. 2002, 19, (1), 73-98.

25. Giteau, A.; Venier-Julienne, M. C.; Aubert-Pouessel, A.; Benoit, J. P. How to achieve sustained and complete protein release from PLGA-based microparticles? Int. J. Pharm. 2008, 350, (1-2), 14-26. 
26. Zhang, Y.; Wischke, C.; Mittal, S.; Mitra, A.; Schwendeman, S. P. Design of controlled release PLGA microspheres for hydrophobic fenretinide. Mol. Pharm. 2016, 13, (8), 2622-30.

27. Wu, D.; Wang, C.; Yang, J.; Wang, H.; Han, H.; Zhang, A.; Yang, Y.; Li, Q. Improving the Intracellular Drug Concentration in Lung Cancer Treatment through the Codelivery of Doxorubicin and miR-519c Mediated by Porous PLGA Microparticle. Mol. Pharm. 2016, 13, (11), 3925-3933.

28. Rui, J.; Dadsetan, M.; Runge, M. B.; Spinner, R. J.; Yaszemski, M. J.; Windebank, A. J.; Wang, H. Controlled release of vascular endothelial growth factor using poly-lactic-co-glycolic acid microspheres: in vitro characterization and application in polycaprolactone fumarate nerve conduits. Acta Biomater. 2012, 8, (2), 511-8.

29. Simon-Yarza, T.; Tamayo, E.; Benavides, C.; Lana, H.; Formiga, F. R.; Grama, C. N.; Ortiz-de-Solorzano, C.; Kumar, M. N.; Prosper, F.; Blanco-Prieto, M. J. Functional benefits of PLGA particulates carrying VEGF and CoQ10 in an animal of myocardial ischemia. Int. J. Pharm. 2013, 454, (2), 784-90.

30. Simon-Yarza, T.; Formiga, F. R.; Tamayo, E.; Pelacho, B.; Prosper, F.; Blanco-Prieto, M. J. PEGylated-PLGA microparticles containing VEGF for long term drug delivery. Int. J. Pharm. 2013, 440, (1), 13-8.

31. Amsden, B. G.; Timbart, L.; Marecak, D.; Chapanian, R.; Tse, M. Y.; Pang, S. C. VEGF-induced angiogenesis following localized delivery via injectable, low viscosity poly(trimethylene carbonate). J. Control. Release 2010, 145, (2), 109-15.

32. Allison, S. D. Analysis of initial burst in PLGA microparticles. Expert Opin Drug Deliv 2008, 5, (6), 615-28.

33. Fredenberg, S.; Wahlgren, M.; Reslow, M.; Axelsson, A. The mechanisms of drug release in poly(lactic-co-glycolic acid)-based drug delivery systems--a review. Int. J. Pharm. 2011, 415, (1-2), 34-52.

34. Fu, K.; Pack, D. W.; Klibanov, A. M.; Langer, R. Visual evidence of acidic environment within degrading poly(lacticco-glycolic acid) (PLGA) microspheres. Pharm. Res. 2000, 17, (1), 100-6.

35. Shenderova, A.; Burke, T. G.; Schwendeman, S. P. The acidic microclimate in poly(lactide-co-glycolide) microspheres stabilizes camptothecins. Pharm. Res. 1999, 16, (2), 241-8.

36. Giteau, A.; Venier-Julienne, M. C.; Marchal, S.; Courthaudon, J. L.; Sergent, M.; Montero-Menei, C.; Verdier, J. M.; Benoit, J. P. Reversible protein precipitation to ensure stability during encapsulation within PLGA microspheres. Eur. J. Pharm. Biopharm. 2008, 70, (1), 127-36.

37. Kazazi-Hyseni, F.; Landin, M.; Lathuile, A.; Veldhuis, G. J.; Rahimian, S.; Hennink, W. E.; Kok, R. J.; van Nostrum, C. F. Computer modeling assisted design of monodisperse PLGA microspheres with controlled porosity affords zero order release of an encapsulated macromolecule for 3 months. Pharm. Res. 2014, 31, (10), 2844-56.

38. Kissel, T.; Li, Y.; Unger, F. ABA-triblock copolymers from biodegradable polyester A-blocks and hydrophilic poly(ethylene oxide) B-blocks as a candidate for in situ forming hydrogel delivery systems for proteins. Adv Drug Deliv Rev 2002, 54, (1), 99-134.

39. Bonacucina, G.; Cespi, M.; Mencarelli, G.; Giorgioni, G.; Palmieri, G. F. Thermosensitive self-assembling block copolymers as drug delivery systems. Polymers-Basel 2011, 3, (2), 779-811.

40. Stankovic, M.; de Waard, H.; Steendam, R.; Hiemstra, C.; Zuidema, J; Frijlink, H. W.; Hinrichs, W. L. Low temperature extruded implants based on novel hydrophilic multiblock copolymer for long-term protein delivery. Eur. J. Pharm. Sci. 2013, 49, (4), 578-87.

41. Stankovic, M.; Hiemstra, C.; de Waard, H.; Zuidema, J.; Steendam, R.; Frijlink, H. W.; Hinrichs, W. L. Protein release from water-swellable poly(D,L-lactide-PEG)-b-poly(-caprolactone) implants. Int. J. Pharm. 2015, 480, (1-2), 7383.

42. Stankovic, M.; Tomar, J.; Hiemstra, C.; Steendam, R.; Frijlink, H. W.; Hinrichs, W. L. Tailored protein release from biodegradable poly(epsilon-caprolactone-PEG)-b-poly(epsilon-caprolactone) multiblock-copolymer implants. Eur. J. Pharm. Biopharm. 2014, 87, (2), 329-37.

43. Sandker, M. J.; Duque, L. F.; Redout, E. M.; Chan, A.; Que, I.; Lowik, C. W.; Klijnstra, E. C.; Kops, N.; Steendam, R.; van Weeren, R.; Hennink, W. E.; Weinans, H. Degradation, intra-articular retention and biocompatibility of monospheres composed of [PDLLA-PEG-PDLLA]-b-PLLA multi-block copolymers. Acta Biomater. 2017, 48, 401414.

44. Chen, W.; Palazzo, A.; Hennink, W. E.; Kok, R. J. Effect of particle size on drug loading and release kinetics of gefitinib-loaded PLGA microspheres. Mol. Pharm. 2017, 14, (2), 459-467. 
45. Berkland, C.; King, M.; Cox, A.; Kim, K.; Pack, D. W. Precise control of PLG microsphere size provides enhanced control of drug release rate. J. Control. Release 2002, 82, (1), 137-47.

46. Vladisavljevic, G. T. Structured microparticles with tailored properties produced by membrane emulsification. Adv. Colloid Interface Sci. 2015, 225, 53-87.

47. Tran, V. T.; Benoit, J. P.; Venier-Julienne, M. C. Why and how to prepare biodegradable, monodispersed, polymeric microparticles in the field of pharmacy? Int. J. Pharm. 2011, 407, (1-2), 1-11.

48. Teekamp, N.; Van Dijk, F.; Broesder, A.; Evers, M.; Zuidema, J.; Steendam, R.; Post, E.; Hillebrands, J. L.; Frijlink, H. W.; Poelstra, K.; Beljaars, L.; Olinga, P.; Hinrichs, W. L. J. Polymeric microspheres for the sustained release of a protein-based drug carrier targeting the PDGFbeta-receptor in the fibrotic kidney. Int. J. Pharm. 2017, 534, (1-2), 229-236.

49. Sah, H. A new strategy to determine the actual protein content of poly(lactide-co-glycolide) microspheres. J. Pharm. Sci. 1997, 86, (11), 1315-8.

50. Ghassemi, A. H.; van Steenbergen, M. J.; Talsma, H.; van Nostrum, C. F.; Crommelin, D. J.; Hennink, W. E. Hydrophilic polyester microspheres: effect of molecular weight and copolymer composition on release of BSA. Pharm. Res. 2010, 27, (9), 2008-17.

51. Ramazani, F.; Hiemstra, C.; Steendam, R.; Kazazi-Hyseni, F.; Van Nostrum, C. F.; Storm, G.; Kiessling, F.; Lammers, T.; Hennink, W. E.; Kok, R. J. Sunitinib microspheres based on [PDLLA-PEG-PDLLA]-b-PLLA multi-block copolymers for ocular drug delivery. Eur. J. Pharm. Biopharm. 2015, 95, (Pt B), 368-77.

52. Petit, A.; Muller, B.; Bruin, P.; Meyboom, R.; Piest, M.; Kroon-Batenburg, L. M.; de Leede, L. G.; Hennink, W. E.; Vermonden, T. Modulating rheological and degradation properties of temperature-responsive gelling systems composed of blends of PCLA-PEG-PCLA triblock copolymers and their fully hexanoyl-capped derivatives. Acta Biomater. 2012, 8, (12), 4260-7.

53. De Vos, R.; Goethals, E. J. End group analysis of commercial poly(ethylene glycol) monomethyl ether's. Polymer Bulletin 1986, 15, (6), 547-549.

54. Ritger, P. L.; Peppas, N. A. A simple equation for description of solute release II. Fickian and anomalous release from swellable devices. J. Control. Release 1987, 5, 37 - 42.

55. Costa, P.; Sousa Lobo, J. M. Modeling and comparison of dissolution profiles. Eur. J. Pharm. Sci. 2001, 13, (2), 123-33.

56. Shah, V. P.; Tsong, Y.; Sathe, P.; Liu, J. P. In vitro dissolution profile comparison--statistics and analysis of the similarity factor, f2. Pharm. Res. 1998, 15, (6), 889-96.

57. Stone, D.; Phaneuf, M.; Sivamurthy, N.; LoGerfo, F. W.; Quist, W. C. A biologically active VEGF construct in vitro: implications for bioengineering-improved prosthetic vascular grafts. J. Biomed. Mater. Res. 2002, 59, (1), 160-5.

58. O'Brien, J.; Wilson, I.; Orton, T.; Pognan, F. Investigation of the Alamar Blue (resazurin) fluorescent dye for the assessment of mammalian cell cytotoxicity. Eur. J. Biochem. 2000, 267, (17), 5421-6.

59. Musilli, C.; Karam, J. P.; Paccosi, S.; Muscari, C.; Mugelli, A.; Montero-Menei, C. N.; Parenti, A. Pharmacologically active microcarriers for endothelial progenitor cell support and survival. Eur. J. Pharm. Biopharm. 2012, 81, (3), 609-16.

60. Samadi, N.; van Steenbergen, M. J.; van den Dikkenberg, J. B.; Vermonden, T.; van Nostrum, C. F.; Amidi, M.; Hennink, W. E. Nanoparticles based on a hydrophilic polyester with a sheddable PEG coating for protein delivery. Pharm. Res. 2014, 31, (10), 2593-604.

61. Huang, M. H.; Li, S.; Hutmacher, D. W.; Schantz, J. T.; Vacanti, C. A.; Braud, C.; Vert, M. Degradation and cell culture studies on block copolymers prepared by ring opening polymerization of epsilon-caprolactone in the presence of poly(ethylene glycol). J. Biomed. Mater. Res. A 2004, 69, (3), 417-27.

62. Petit, A.; Muller, B.; Meijboom, R.; Bruin, P.; van de Manakker, F.; Versluijs-Helder, M.; de Leede, L. G.; Doornbos, A.; Landin, M.; Hennink, W. E.; Vermonden, T. Effect of polymer composition on rheological and degradation properties of temperature-responsive gelling systems composed of acyl-capped PCLA-PEG-PCLA. Biomacromolecules 2013, 14, (9), 3172-82.

63. Zweers, M. L.; Engbers, G. H.; Grijpma, D. W.; Feijen, J. In vitro degradation of nanoparticles prepared from polymers based on DL-lactide, glycolide and poly(ethylene oxide). J. Control. Release 2004, 100, (3), $347-56$.

64. Ghaderi, R. S., C.; Carlfors, J. Effect of preparative parameters on the characteristics ofpoly (D, L-lactide-coglycolide) microspheres made by the double emulsion method. 1996. 
65. Ito, F.; Fujimori, H.; Honnami, H.; Kawakami, H.; Kanamura, K.; Makino, K. Control of drug loading efficiency and drug release behavior in preparation of hydrophilic-drug-containing monodisperse PLGA microspheres. J. Mater. Sci. Mater. Med. 2010, 21, (5), 1563-71.

66. Ito, F.; Fujimori, H.; Makino, K. Incorporation of water-soluble drugs in PLGA microspheres. Colloids Surf. B. Biointerfaces 2007, 54, (2), 173-8.

67. Liu, R.; Huang, S. S.; Wan, Y. H.; Ma, G. H.; Su, Z. G. Preparation of insulin-loaded PLA/PLGA microcapsules by a novel membrane emulsification method and its release in vitro. Colloids Surf. B. Biointerfaces 2006, 51, (1), 30-8.

68. Formiga, F. R.; Pelacho, B.; Garbayo, E.; Abizanda, G.; Gavira, J. J.; Simon-Yarza, T.; Mazo, M.; Tamayo, E.; Jauquicoa, C.; Ortiz-de-Solorzano, C.; Prosper, F.; Blanco-Prieto, M. J. Sustained release of VEGF through PLGA microparticles improves vasculogenesis and tissue remodeling in an acute myocardial ischemia-reperfusion model. J. Control. Release 2010, 147, (1), 30-7.

69. Teekamp, N.; Duque, L. F.; Frijlink, H. W.; Hinrichs, W. L.; Olinga, P. Production methods and stabilization strategies for polymer-based nanoparticles and microparticles for parenteral delivery of peptides and proteins. Expert Opin Drug Deliv 2015, 12, (8), 1311-31.

70. Fonte, P.; Andrade, F.; Azevedo, C.; Pinto, J.; Seabra, V.; van de Weert, M.; Reis, S.; Sarmento, B. Effect of the freezing step in the stability and bioactivity of protein-loaded PLGA nanoparticles upon lyophilization. Pharm. Res. 2016, 33, (11), 2777-93.

71. Fonte, P.; Araujo, F.; Seabra, V.; Reis, S.; van de Weert, M.; Sarmento, B. Co-encapsulation of lyoprotectants improves the stability of protein-loaded PLGA nanoparticles upon lyophilization. Int. J. Pharm. 2015, 496, (2), 850-62.

72. Singh, S. K.; Kolhe, P.; Mehta, A. P.; Chico, S. C.; Lary, A. L.; Huang, M. Frozen state storage instability of a monoclonal antibody: aggregation as a consequence of trehalose crystallization and protein unfolding. Pharm. Res. 2011, 28, (4), 873-85.

73. Bhatnagar, B. S.; Bogner, R. H.; Pikal, M. J. Protein stability during freezing: separation of stresses and mechanisms of protein stabilization. Pharm. Dev. Technol. 2007, 12, (5), 505-23.

74. Borselli, C.; Ungaro, F.; Oliviero, O.; d'Angelo, I.; Quaglia, F.; La Rotonda, M. I.; Netti, P. A. Bioactivation of collagen matrices through sustained VEGF release from PLGA microspheres. J. Biomed. Mater. Res. A 2010, 92, (1), 94-102. 


\section{Supporting Information}

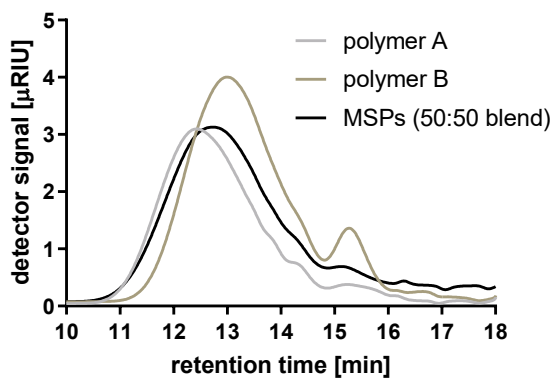

FIGURE S1: GPC chromatograms of multiblock copolymers A and B ("polymer A" and "polymer B"), and of (placebo) microspheres prepared with a 50:50 blend of polymer A and B (MSPs: microspheres).

TABLE S1: Molecular weights of multiblock copolymers A and B and placebo microspheres.

\begin{tabular}{ccccc}
\hline & $\mathbf{M}_{\mathbf{n}}[\mathbf{k D a}]$ & $\mathbf{M}_{\mathbf{w}}[\mathbf{k D a}]$ & $\mathbf{M}_{\mathbf{p}}[\mathbf{k D a}]$ & $\begin{array}{c}\text { PDI } \\
{\left[\mathbf{M}_{\mathbf{w}} / \mathbf{M}_{\mathbf{n}}\right]}\end{array}$ \\
\hline polymer A & 21.7 & 53.7 & 52.4 & 2.59 \\
polymer B & 13.7 & 31.6 & 30.5 & 2.31 \\
placebo microspheres (50:50 blend) & 17.2 & 45.6 & 39.1 & 2.64 \\
\hline
\end{tabular}

Polymer A: multiblock copolymer A, Polymer B: multiblock copolymer B. Placebo microspheres (50:50 blend): Microspheres prepared with a 50:50 blend of polymer A and B. Molecular weights are based on GPC measurements (as shown in figure S1).

TABLE S2: Size characteristics of VEGF-loaded microspheres ( $0.2 \mathrm{wt} \%$ target loading) prepared with various polymer $A$ and $B$ blends.

\begin{tabular}{cc}
\hline $\begin{array}{c}\text { blend ratio } \\
\text { polymer A : polymer B }\end{array}$ & average size $[\boldsymbol{\mu m}] ; \mathbf{C V}[\%]$ \\
\hline $100: 0$ & $45 ; 14$ \\
$90: 10$ & n.d.* \\
$80: 20$ & $46 ; 28$ \\
$70: 30$ & $51 ; 12$ \\
$60: 40$ & $42 ; 16$ \\
$50: 50$ & $48 ; 21$ \\
$0: 100$ & $43 ; 28$ \\
\hline
\end{tabular}

*not determined. 

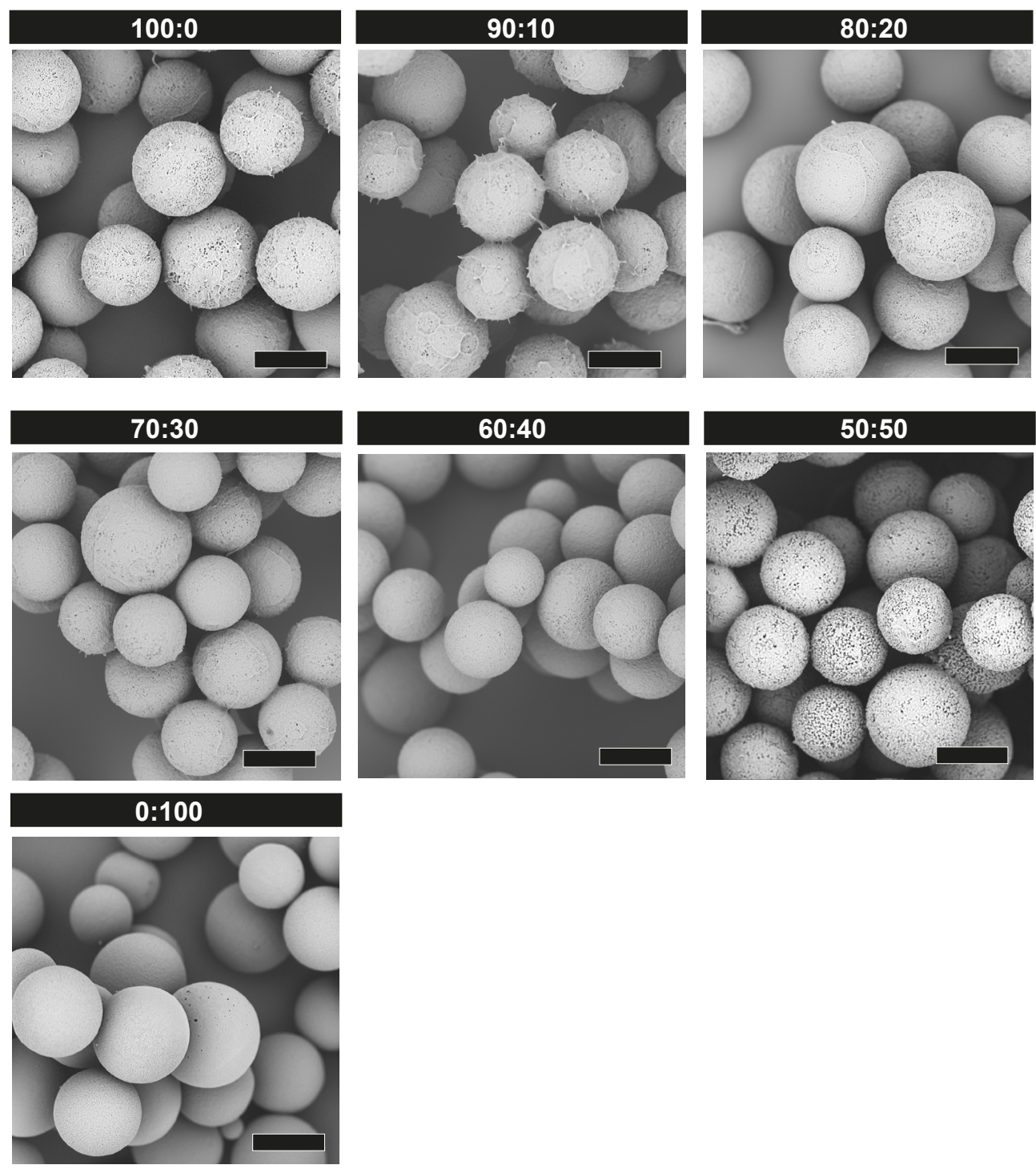

FIGURE S2: SEM images of VEGF-loaded microspheres ( $0.2 \mathrm{wt} \%$ target loading). The blend ratio of polymer A and B used for each batch is indicated on the bar above each image. Black scale bar represents $30 \mu \mathrm{m}$. 


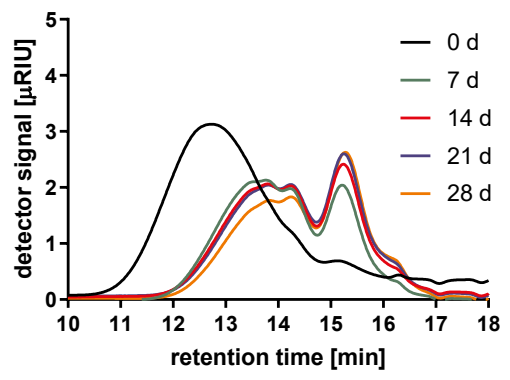

FIGURE S3: GPC chromatograms of (placebo) microsphere degradation samples. The time of incubation in IVR buffer (PBS pH 7.4, supplemented with 0.025\% Tween 20 and $0.02 \% \mathrm{NaN}_{3}$ ) at $37^{\circ} \mathrm{C}$ is indicated in the legend (d: days). The GPC chromatrogram of freshly prepared microspheres is shown in black ("0 d").
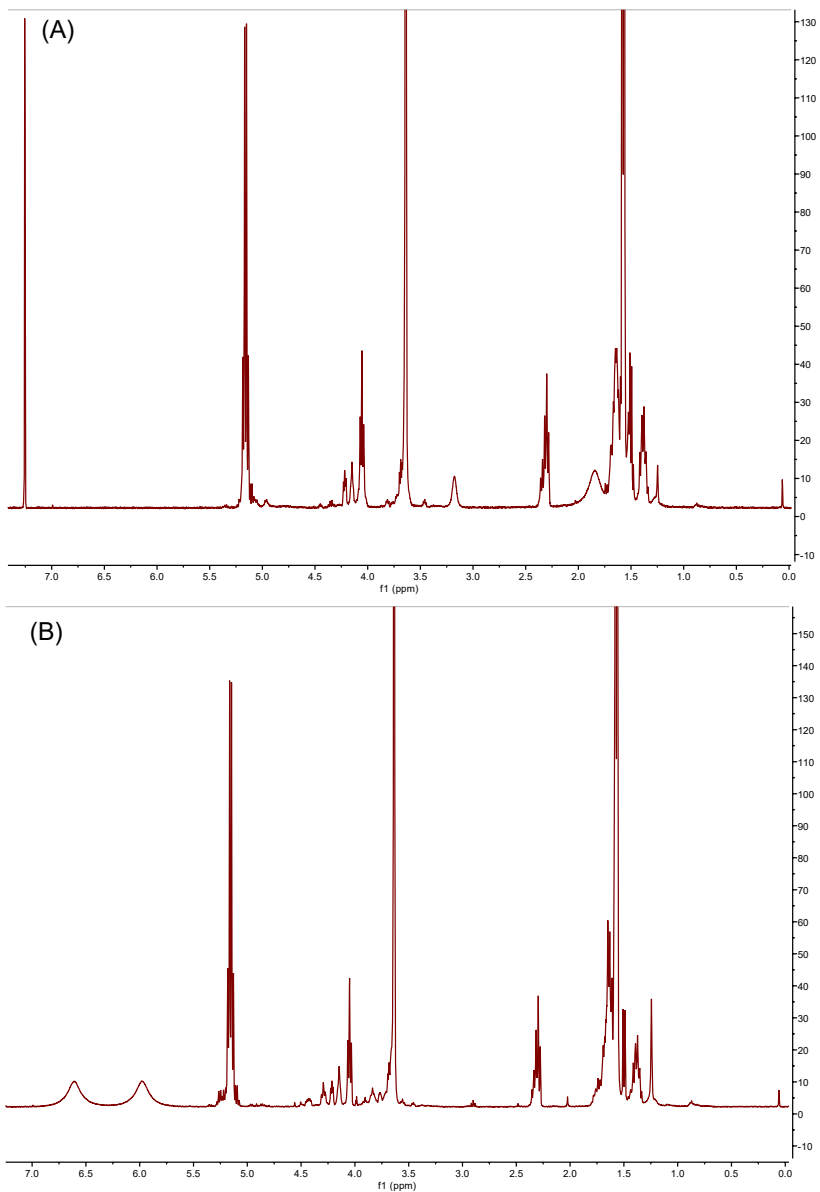

FIGURE S4: ${ }^{1} \mathrm{H}-\mathrm{NMR}$ spectra of freeze-dried placebo microspheres directly after preparation (A). Figure B: After addition of shift reagent TAIC a small additional peak at $\sim \delta 4.5$ is visible. 

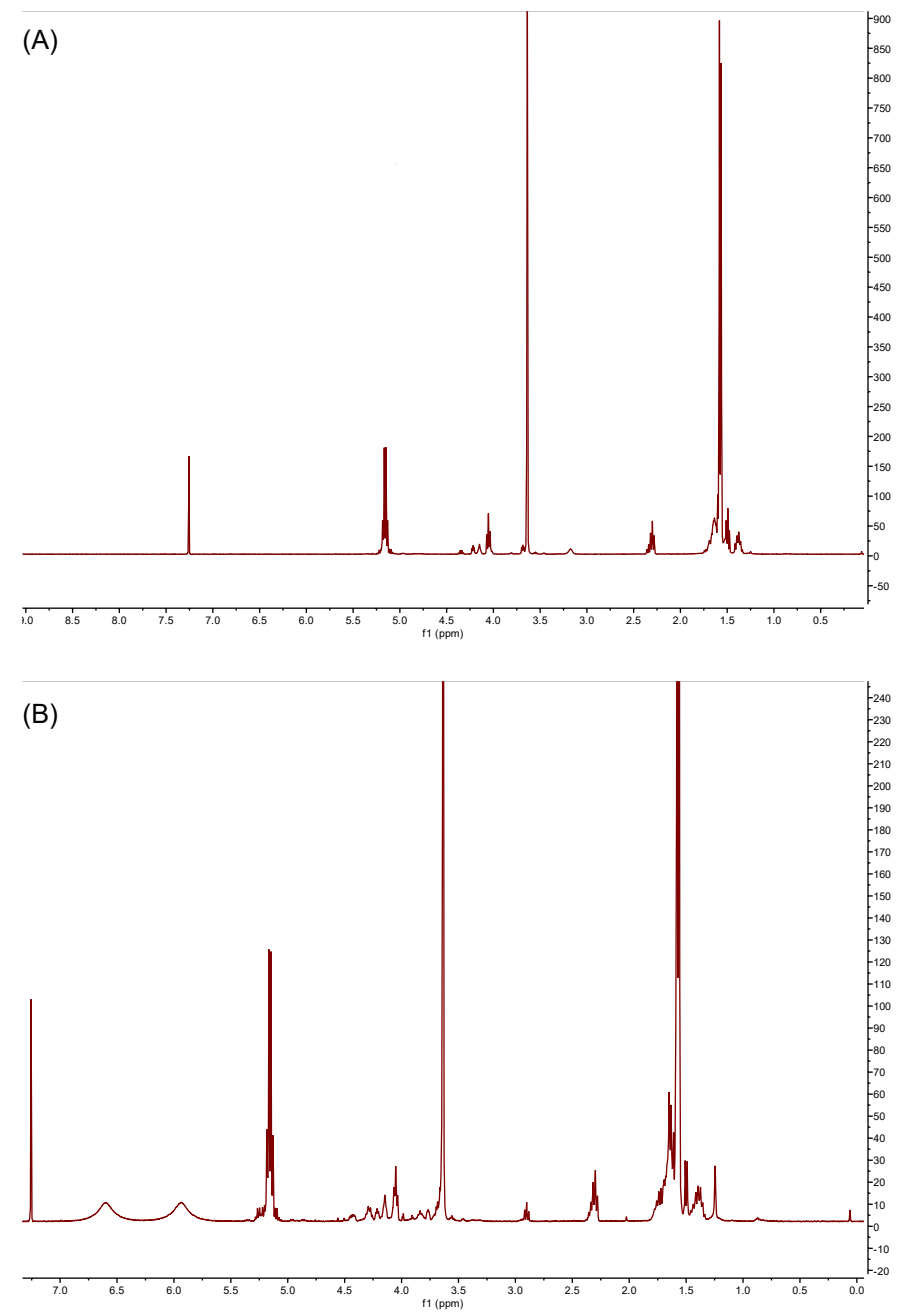

FIGURE S5: 'H-NMR spectra of freeze-dried placebo microspheres after incubation in IVR buffer (PBS pH 7.4, 0.025\% Tween $20,0.02 \% \mathrm{NaN}_{3}$ ) at $37^{\circ} \mathrm{C}$ for 28 days (figure A). Figure B: After addition of shift reagent TAIC a small additional peak at $\sim \delta 4.5$ is visible. 
Batch 1

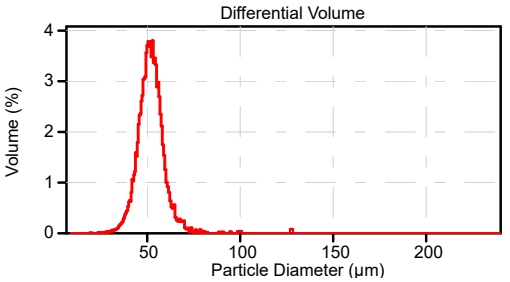

Batch 2

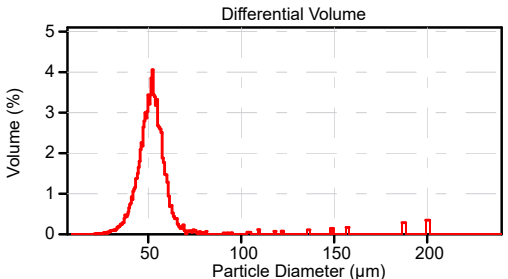

\section{Batch 3}

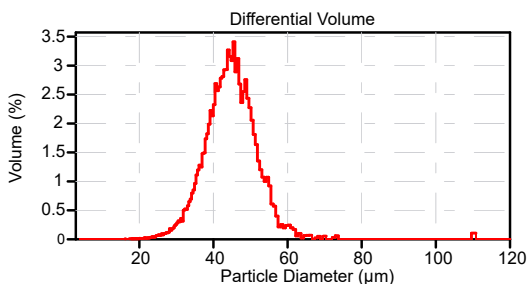

FIGURE S6: Size distribution plots, measured by Coulter Counter, of 3 microsphere batches prepared with 50:50 blend ratio of polymer $\mathrm{A}$ and $\mathrm{B}, 1 \mathrm{wt} \%$ target $\mathrm{VEGF}$ loading.

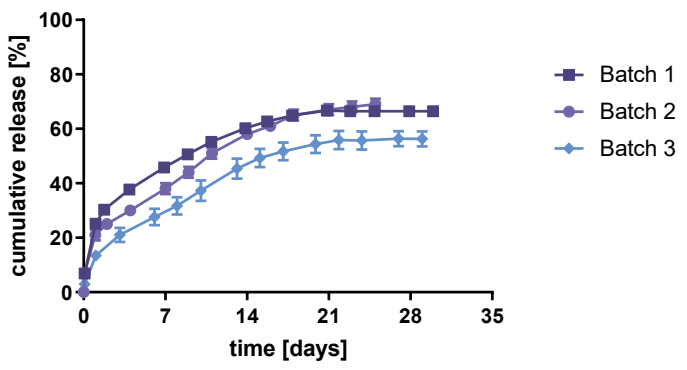

FIGURE S7: Cumulative release ofVEGF from three microsphere batches (50:50 blend ratio polymer $\mathrm{A}$ and $\mathrm{B}$ ) prepared with a target VEGF loading of $1 \mathrm{wt} \%$. The release was performed at $37^{\circ} \mathrm{C}$ in IVR buffer (PBS pH 7.4, supplemented with $0.025 \%$ Tween 20 and $0.02 \% \mathrm{NaN}_{3}$ ). Released VEGF was quantified by SE-UPLC.

TABLE S3: VEGF quantification by SE-UPLC and ELISA.

\begin{tabular}{ccc}
\hline \multirow{2}{*}{ theoretical VEGF concentration [ $\boldsymbol{\mu g} / \mathbf{m l}]$} & \multicolumn{2}{c}{ measured VEGF concentration $[\boldsymbol{\mu g} / \mathbf{m l}]$} \\
\cline { 2 - 3 } & SE-UPLC & ELISA \\
\hline 6 & $6 \pm 0$ & $4 \pm 0$ \\
60 & $60 \pm 1$ & $44 \pm 2$ \\
160 & $158 \pm 0$ & $92 \pm 3$ \\
\hline
\end{tabular}

Theoretical concentrations $[\mu \mathrm{g} / \mathrm{ml}]$ are based on the amount of lyophilized VEGF per vial, as received by the supplier. The content of a vial (1 mg lyophilized VEGF) was reconstituted in $1 \mathrm{ml}$ IVR buffer, resulting in a $1 \mathrm{mg} / \mathrm{ml} \mathrm{VEGF} \mathrm{solution.} \mathrm{This} \mathrm{solution} \mathrm{was} \mathrm{further} \mathrm{diluted} \mathrm{to} \mathrm{the}$ concentrations stated in this table. 
TABLE S4: Quantification of released and freshly spiked VEGF by SE-UPLC and ELISA.

\begin{tabular}{cccc}
\hline \multirow{2}{*}{ IVR sample } & VEGF spike $[\boldsymbol{\mu g} / \mathbf{m l}]$ & \multicolumn{2}{c}{ measured VEGF concentration $[\boldsymbol{\mu g} / \mathbf{m l}]$} \\
\cline { 3 - 4 } & & SE-UPLC & ELISA \\
\hline \multirow{2}{*}{ Day 1 } & 0 & $18 \pm 0$ & $5 \pm 1$ \\
& 50 & $70 \pm 1$ & $45 \pm 4$ \\
Day 11 & 0 & $8 \pm 0$ & $3 \pm 0$ \\
& 50 & $57 \pm 0$ & $32 \pm 1$ \\
Day 18 & 0 & $5 \pm 0$ & $2 \pm 0$ \\
& 50 & $55 \pm 1$ & $38 \pm 9$ \\
\hline
\end{tabular}
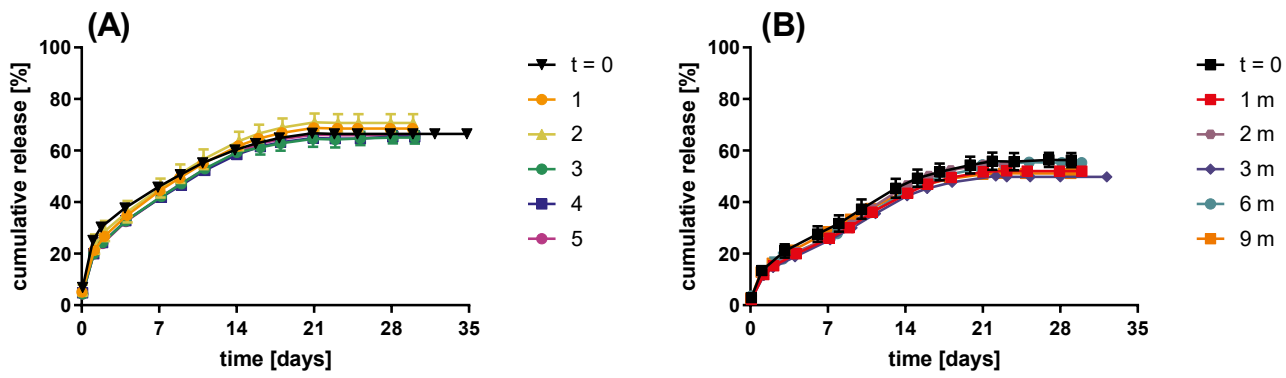

FIGURE S8: Cumulative release of VEGF [\%] from freeze-dried microspheres. A: after repeated freezing and storage $-20^{\circ} \mathrm{C}$, B: after long-term storage at $-20^{\circ} \mathrm{C}$ for $1,2,3,6$ months compared to directly after freeze-drying $(t=0)$. The release was performed at $37^{\circ} \mathrm{C}$ in PBS pH 7.4, supplemented with $0.025 \%$ Tween 20 and $0.02 \% \mathrm{NaN}_{3}$. Released VEGF was quantified by SE-UPLC.

TABLE S5: Similarity factor $f_{2}$ of VEGF release curves from microspheres after 1 to 5 times of freezing to and storage at $-20^{\circ} \mathrm{C}$ compared to VEGF release curve from microspheres after preparation and freeze-drying.

\begin{tabular}{cc}
\hline freezing cycles & $\boldsymbol{f}_{\mathbf{2}}$ \\
\hline 1 & 81 \\
2 & 74 \\
3 & 74 \\
4 & 74 \\
5 & 75 \\
\hline
\end{tabular}


TABLE S6: Similarity factor $f_{2}$ of VEGF release curves from microspheres after various months of storage at $-20^{\circ} \mathrm{C}$ and VEGF release curves from microspheres after preparation and freeze-drying (batch 3).

\begin{tabular}{cl}
\hline storage time & $\boldsymbol{f}_{2}$ \\
\hline 1 month & 63 \\
2 months & 68 \\
3 months & 59 \\
6 months & 73 \\
9 months & 65 \\
\hline
\end{tabular}

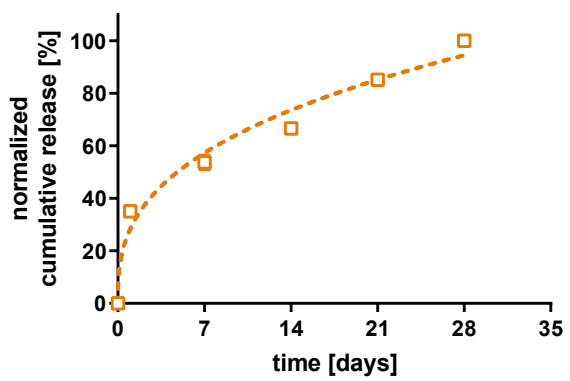

FIGURE S9: Normalized cumulative release profiles of VEGF from microspheres. Release was performed at $37^{\circ} \mathrm{C}$ in bioactivity IVR buffer (PBS pH 7.4, supplemented with 0.5\% BSA, $30 \mu \mathrm{g} / \mathrm{ml}$ and $15 \mathrm{ng} / \mathrm{ml}$ amphotericin). KorsmeyerPeppas model fitting (orange dotted line): correlation coefficient $\left(R^{2}\right)$ : 0.98, Diffusional exponent $(n): 0.36 \pm 0.05,95 \%$ confidence interval: $0.22-0.55$.

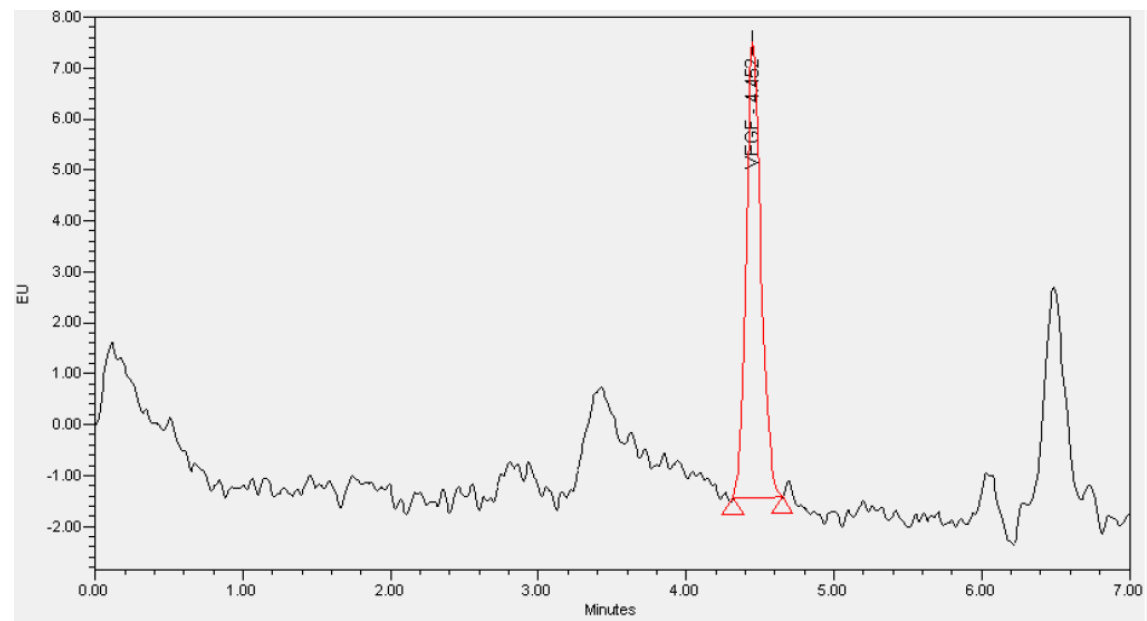

FIGURE S10: Representative SE-UPLC chromatogram for VEGF quantification. Peaks at 3.5 minutes, 6 minutes and 6.5 minutes have not been identified. 


\title{
Chapter 3
}

\section{Vascular Endothelial Growth Factor-releasing Microspheres based on Poly( $\varepsilon$-caprolactone-PEG- $\varepsilon$-caprolactone)-b-poly(L- lactide) Multiblock Copolymers incorporated in a 3D-printed Poly(dimethylsiloxane) Cell Macroencapsulation Device}

\author{
Karina C. Scheiner', Fergal Coulter ${ }^{2,3}$, Roel F. Maas-Bakker', Giulio Chersi ${ }^{4,5}$, \\ Thanh T. Nguyen ${ }^{6}$, Rob Steendam ${ }^{6}$, Garry P. Duffy ${ }^{7}$, Wim E. Hennink', \\ Eoin D. O'Cearbhaill'², Robbert ]. Kok'
}

'Department of Pharmaceutics, Utrecht Institute for Pharmaceutical Sciences, Utrecht University, Universiteitsweg 99, 3584 CG Utrecht, the Netherlands ${ }^{2}$ UCD Centre for Biomedical Engineering, School of Mechanical and Materials Engineering,

University College Dublin, Engineering Building Belfield Dublin 4, Dublin, Ireland ${ }^{3}$ Department of Complex Materials, ETH Zurich, Vladimir-Prelog-Weg 1-5/10, 8093 Zürich,

Switzerland

${ }^{4}$ ABIEL s.r.I c/o ARCA Incubatore di Imprese, viale delle Scienze Ed.16, 90128 Palermo, Italy

${ }^{5}$ Dipartimento di Scienze e Tecnologie Biologiche, Chimiche e Farmaceutiche, Università degli studi di Palermo - viale delle Scienze Ed.16, 90128 Palermo, Italy

${ }^{6}$ InnoCore Pharmaceuticals B.V., L.J. Zielstraweg 1, 9713 GX Groningen, the Netherlands

${ }^{7}$ Discipline of Anatomy, School of Medicine, National University of Ireland Galway,

University Road, Galway, Ireland 


\section{Abstract}

Pancreatic islet transplantation is a promising advanced therapy that has been used to treat patients suffering from diabetes type 1. Traditionally, pancreatic islets are infused via the portal vein which are subsequently intended to engraft in the liver. Severe immunosuppressive treatments are necessary, however, to prevent rejection of the transplanted islets. Novel approaches therefore have focused on encapsulation of the islets in biomaterial implants which can protect the islets and offer an organ-like environment. Vascularization of the device's surface is a prerequisite for the survival and proper functioning of transplanted pancreatic islets. We are pursuing a prevascularization strategy by incorporation of vascular endothelial growth factor (VEGF) loaded microspheres in 3D-printed poly(dimethylsiloxane) (PDMS)based devices prior to their prospective loading with transplanted cells. Microspheres $(\sim 50$

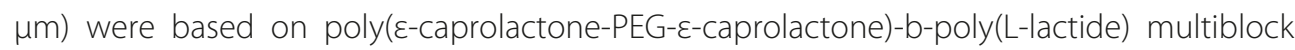
copolymers and were loaded with $10 \mathrm{\mu g} \mathrm{VEGF} / \mathrm{mg}$ microspheres, and subsequently dispersed in a hyaluronic acid carrier liquid. In vitro release studies at $37^{\circ} \mathrm{C}$ demonstrated continuous release of fully bioactive VEGF for four weeks. In conclusion, our results demonstrate that incorporation of VEGF-releasing microspheres ensures adequate release of VEGF for a time window of four weeks, which is attractive in view of the vascularization of artificial pancreas implants. 


\section{Introduction}

Patients with juvenile diabetes (diabetes type 1) suffer from inadequate insulin production, which often relates to autoimmune destruction of the pancreatic beta-cells.' Although blood glucose homeostasis can be restored by injection of insulin, novel advanced therapies are being pursued that can restore insulin production. Transplantation of healthy insulin-secreting pancreatic islets is a promising treatment for diabetes type 1 that has been studied since the 1960s. ${ }^{2}$ Traditionally, pancreatic islets are infused into the portal vein and become engrafted in the microvasculature of the liver. However, poor islet survival, rejection by the host's immune system and the need to use strong immunosuppressing drugs have restricted this treatment's widespread use. ${ }^{3}$ Another issue is the lack of donors for pancreatic islet transplantation which is especially severe since on average three donors are needed per recipient. ${ }^{2,4}$ Strategies that can improve the efficiency of islet transplantation and the overall survival of islets are therefore warranted. Encapsulating pancreatic islets into a biomaterial device is an attractive approach, as it provides a physical barrier between the transplanted islets and the host's immune cells, creating an immune-deprived organ-like environment. ${ }^{5}$ Pancreatic islet encapsulation devices have been developed for subcutaneous implantation and have even advanced into phase I-II clinical testing. ., $^{6}$ In some cases, devices are pre-filled with islets before implantation while other devices include filling ports, offering islet replenishment. ${ }^{7}$ Since the formation of blood vessels is vital for transport of oxygen and nutrients, neovascularization of the device remains the key challenge for survival of pancreatic islets after transplantation; ${ }^{8}$ moreover, vascularization of the device is also needed for transport of released insulin into the circulation and beyond., ${ }^{3,5}$ Several approaches seem feasible, among others coating of the polymeric implants with compounds such as fibrin and platelet-rich plasma to mimic the extracellular matrix, or coformulation of proangiogenic growth factors within the device, 9,10 for instance vascular endothelial growth factor (VEGF). ${ }^{11-13}$ Proper functional vascularization of the surface of implants, i.e. the formation of stable blood vessels that encompass the device, will take around four weeks upon implantation, depending on the site of implantation, as shown in rodent models $s^{10,14,15}$. We therefore postulate that continuous delivery of VEGF for three to four weeks is preferred and that such a sustained release can best be achieved by its encapsulation in a controlled drug delivery systems (DDS) such as microspheres. ${ }^{16,17}$ The most commonly used polymer for the preparation of microspheres, poly(lactic-co-glycolic acid) (PLGA), ${ }^{18-20}$ has several drawbacks regarding the release profile and stability of encapsulated proteins. ${ }^{21,}$ 22 The release of encapsulated cargo is often biphasic, i.e. a high burst release followed by a relatively slow release, ${ }^{20,23,24}$ and is thus not a preferred release profile for vascularization of implants. ${ }^{15,25}$ Further, the acidic degradation products of PLGA-based systems cause a pH decrease within the polymeric matrix, ${ }_{1}^{26,27}$ and therefore negatively impact the stability of proteins. ${ }^{21,28,29}$ We therefore evaluated a different kind of biodegradable polymer composed of multiblock copolymers with alternating hydrophilic and hydrophobic blocks ${ }^{30,31}$ for the encapsulation of VEGF in microspheres. These multiblock copolymers form amorphous 
domains (primarily consisting of poly( $\varepsilon$-caprolactone) - poly(ethylene glycol) - poly( $\varepsilon$ caprolactone) blocks) and semi-crystalline domains (mainly composed of poly(L-lactic acid) blocks), and are attractive for protein delivery due to their well-controlled swelling properties which allow continuous release through diffusion with low burst release. ${ }^{32-35}$ The release of proteins can be tailored by the weight fraction and molecular weight of PEG, ${ }^{33,36}$ which was also demonstrated for microspheres loaded with VEGF. ${ }^{35}$ In this study, we incorporated VEGF-loaded microspheres in poly(dimethylsiloxane) (PDMS)-based devices and studied the release of VEGF and its bioactivity. Hyaluronic acid solution served as carrier liquid for VEGF-loaded microspheres. Both PDMS and hyaluronic acid have been reported as suitable biomaterials for pancreatic islets transplantation systems. ${ }^{37-39}$ The current PDMS devices were prepared by 3D-printing, an arising technology in the biomaterial field which can produce well-defined reproducible scaffolds. ${ }^{40}$ PDMS membranes are highly gas permeable, however, they are also required to facilitate the transportation of larger molecules, such as proteins, for cell microencapsulation applications. ${ }^{5}$ Therefore, micrometer-sized pores were created by inclusion of a porogen, which, after its extraction, results in a permeable structure through which VEGF can diffuse into the surrounding fluids. We characterized the devices by scanning electron microscopy and studied the distribution of red-labelled microspheres within the device. The in vitro release of VEGF from PDMS devices filled with VEGF microspheres was studied by ELISA. The bioactivity of released VEGF was demonstrated by a HUVEC-proliferation based bioassay.

\section{Experimental section}

\section{Materials}

PDMS devices were prepared by 3D-printing using silicone rubber elastomer (PDMS) Nusil MED-4840 (Avantor, Radnor, PA, USA), ${ }^{\text {a } 41}$ Hyaluronic acid (HA) and FITC-labelled HA (both 1.26 $\mathrm{MDa}$ ) were supplied by Contipro (Prague, Czech Republic). Recombinant human vascular endothelial growth factor (VEGF) 165 was purchased from Peprotech Inc (Rocky Hill, NJ, USA). PLGA (50:50, i.v. $0.4 \mathrm{dL} / \mathrm{g}$ ) was purchased from Corbion (Gorinchem, the Netherlands). PVA (87 - 90 \% hydrolyzed, average mol wt 30,000 - 70,000), Tween 20, sodium chloride and fluorescein sodium salt were purchased from Sigma Aldrich (Zwijndrecht, the Netherlands). Dichloromethane was purchased from Biosolve Chimie (Dieuze, France). Blue Dextran 2000 (2 MDa) was purchased from GE Healthcare Bio-Sciences AB (Uppsala, Sweden). Nile Red was purchased from Carl Roth (Karlsruhe, Germany). Gibco Dulbecco's Phosphate Buffered Saline (DPBS, 10x, composition 27mM KCl, 15mM H $\mathrm{PO}_{4^{\prime}} 1.4 \mathrm{M} \mathrm{NaCl}, 81 \mathrm{mM} \mathrm{Na}_{2} \mathrm{HPO}_{4} \times 7 \mathrm{H}_{2} \mathrm{O}$ ) and propidium iodide were purchased from Thermo Fisher Scientific (the Netherlands). Collagen I (from rat tail) was obtained from Corning (Corning, NY, USA). Calcein AM was purchased from Cayman Chemical Company (Ann Arbor, MI, USA). 


\section{Methods}

\section{Preparation of study components}

Preparation of hyaluronic acid (HA)-based carrier liquid

Hyaluronic acid was dissolved in 10 ml PBS pH 7.4 (Dulbecco's PBS, final concentration 10 $\mathrm{mg} / \mathrm{ml}$ ) by stirring under mild heating ( $\sim 0^{\circ} \mathrm{C}$ ) for 16 hours. After complete dissolution (determined visually), the viscous solution was autoclaved (program: $120^{\circ} \mathrm{C}, 2$ bar, 21 minutes, autoclave: Zirbus Technology, Benelux BV, Tiel, the Netherlands) and cooled down to room temperature. HA carrier liquid was stored at $4^{\circ} \mathrm{C}$.

\section{Preparation of microspheres}

VEGF-loaded and placebo microspheres were prepared with Synbiosys multiblock copolymers using a membrane emulsification-based double emulsion method and characterized as reported previously.35 Microspheres were prepared with a 50:50 blend of Synbiosys multiblock copolymers A and B. Polymer A ( $54 \mathrm{kDa})$ consisted of $30 \mathrm{wt} \%$ of an amorphous,

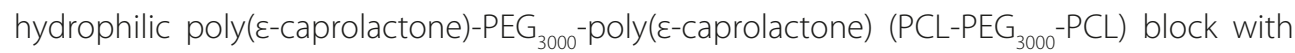
a molecular weight of $4,000 \mathrm{~g} / \mathrm{mol}$ and $70 \mathrm{wt} \%$ of a semi-crystalline poly(L-lactide) (PLLA) block with a molecular weight of $4,000 \mathrm{~g} / \mathrm{mol}$. The total PEG weight fraction of polymer $\mathrm{A}$ is $22.5 \%$. Polymer B ( $32 \mathrm{kDa})$ consisted of $50 \mathrm{wt} \%$ of PCL-PEG ${ }_{1000}-\mathrm{PCL}\left(\mathrm{M}_{\mathrm{w}}: 2,000 \mathrm{~g} / \mathrm{mol}\right)$ and $50 \mathrm{wt} \%$ of poly(L-lactide) block ( $\left.\mathrm{M}_{\mathrm{w}}: 4,000 \mathrm{~g} / \mathrm{mol}\right)$, resulting in a PEG weight fraction of $25 \%$. The total PEG content [\%] of the 50:50 polymer blend is $23.8 \%$. In brief, $1 \mathrm{ml}$ of a solution of VEGF (15 - $20 \mathrm{mg} / \mathrm{ml}$ in $5 \mathrm{mM}$ sodium succinate buffer $\mathrm{pH}$ 5) was dispersed in a $10 \mathrm{ml}$ of a multiblock copolymer solution (15 - 20wt\% in DCM) by Ultra-turrax homogenization (T25 Basic, IKA, Wilmington, USA; 40 seconds at 21,600 rpm). The resulting primary emulsion was pressed through $20 \mu \mathrm{m}$ pores of a stainless steel membrane ( $20 \mu \mathrm{m} \times 200 \mu \mathrm{m}$ Hydrophilic Ringed Stainless-Steel Membrane, Micropore Technologies, Redcar, United Kingdom) into a continuous phase (4 wt\% PVA, $5 \mathrm{wt} \% \mathrm{NaCl}$ ). The secondary emulsion was stirred at $200 \mathrm{rpm}$ with a magnetic stirrer for 3 hours to evaporate the organic solvent. Obtained microspheres were collected on a $5 \mu \mathrm{m}$ filter and washed three times with water. After lyophilization, microspheres were stored at $-20^{\circ} \mathrm{C}$. Nile Red-labelled placebo microspheres were prepared by membrane emulsification of a 10 wt\% PLGA solution supplemented with $1 \mathrm{mg} / \mathrm{ml}$ Nile Red (conventional PLGA was used since these microspheres were only used for visual display of particle penetration within the devices). The average size distribution of the microspheres was measured with an optical particle sizer (Accusizer 780, Santa Barbara, CA, USA). A size distribution plot is shown in figure S1.

\section{Preparation of microsphere dispersions in HA carrier liquid}

Microsphere dispersions in 1\% HA carrier liquid were prepared by stirring a defined amount of microspheres in a corresponding amount of HA carrier liquid with a thin spatula until a homogeneous dispersion was obtained. 


\section{Characterization of PDMS devices}

\section{Scanning electron microscopy}

The surface morphology of the prepared PDMS devices was visualized by scanning electron microscopy (SEM). PDMS devices were submerged in deionized water and sonicated at $37^{\circ} \mathrm{C}$ three times for 30 minutes to remove porogen (sodium chloride) crystals. Next, the devices were dried under vacuum at room temperature for 16 hours. Slices of devices were cut with a razor blade and placed on stubs with double-sized carbon tape. The samples were coated with a thin platinum layer and imaged with SEM (Phenom, FEl company, the Netherlands). The diameters of pores were measured with ImageJ (NIH, Bethesda, MD, USA).

\section{Filling of PDMS devices}

Dry devices were wetted and washed by submerging the devices in $20 \mathrm{ml}$ deionized water and sonicating for 30 minutes at $37^{\circ} \mathrm{C}$ three times. After each sonification step, the water was replaced. Freshly prepared solutions or microsphere dispersions in 1\% HA carrier liquid (as described in sections Release of Fluorescein, Blue Dextran and FITC-HA filled in PDMS devices and Release of VEGF filled in PDMS devices) were transferred into a $1 \mathrm{ml}$ syringe. A target volume of $160 \mu \mathrm{l}$ was injected into pre-wetted devices via a 18G needle. The weight of the syringe and needle was noted before and after filling of the devices. The inlet of the device was closed with a custom-made stopper printed using FormLabs Form 2 Dental SG resin (Formlabs, Somerville, MA, USA). Proper distribution of microspheres within the devices was visualized by filling with a $12 \mathrm{mg} / \mathrm{ml}$ dispersion of Nile Red-labelled microspheres in HA carrier liquid. Since Nile Red is not released from the microspheres, the red color in photographs represents the dispersion of microspheres within the lumen of the devices.

\section{HUVEC live/dead biocompatibility assay}

The toxicity of leachables from PDMS devices filled with HA carrier liquid was evaluated by a live/dead assay with human umbilical vein endothelial cells (HUVECs). Washed PDMS devices were filled with $160 \mu \mathrm{l}$ of HA carrier liquid (as described in section Filling of PDMS devices, no microspheres present), transferred into a glass vial containing $10 \mathrm{ml}$ of cell-compatible IVR buffer (Dulbecco's PBS pH 7.4, 0.2 um filtered, 0.5\% BSA, $30 \mu \mathrm{g} / \mathrm{ml}$ gentamicin and $15 \mathrm{ng} /$ $\mathrm{ml}$ amphotericin) and incubated at $37^{\circ} \mathrm{C}$ under mild agitation. After 1, 2, 3 and 4 weeks of incubation, the complete supernatant was removed and replaced with fresh cell-compatible IVR buffer. The collected supernatants were diluted 20 times in HUVEC complete medium (endothelial cell basal medium 2 (C-22211) supplemented with endothelial cell growth medium 2 supplement mix (C-39216), both obtained from Sigma Aldrich, Zwijndrecht, the Netherlands). Blank complete culture medium, cell-compatible IVR buffer diluted 20 times in complete medium, and $8 \mu \mathrm{g} / \mathrm{ml} \mathrm{HA}$ in complete medium containing 20 times diluted cellcompatible IVR buffer (which corresponds to the concentration of HA in filled devices) served as controls. Prior to the experiment, 96-well plates were coated overnight at $4{ }^{\circ} \mathrm{C}$ with $100 \mathrm{ul}$ 
of collagen coating solution ( $50 \mu \mathrm{g} / \mathrm{ml}$ collagen in $20 \mathrm{mM}$ acidic acid). Afterwards, plates were washed twice with PBS before seeding of the cells. Wells were filled with $100 \mu \mathrm{l}$ of sample or controls and 4000 cells suspended in $100 \mathrm{ul}$ of complete medium (200 $\mu$ l total volume per well). Cells were incubated at $37{ }^{\circ} \mathrm{C} / 5 \% \mathrm{CO}_{2}$ for 24 hours. Live/dead staining was made by incubating with Calcein AM and propidium iodide working solution (prepared according to the supplier's instructions) for 20 minutes. Images were taken with a fluorescent confocal microscope (Yokogawa Cell Voyager CV7000, Tokyo, Japan; Calcein AM (green signal): $\lambda_{\text {ex }} 490$ $\mathrm{nm}, \lambda_{\mathrm{em}} 515 \mathrm{~nm}$; propidium iodine (red signal): $\lambda_{\mathrm{ex}} 535 \mathrm{~nm}, \lambda_{\mathrm{em}} 617 \mathrm{~nm}$ ) and analyzed with Columbus image analysis software (PerkinElmer, Waltham, MA, USA).

\section{Release of Fluorescein, Blue Dextran and FITC-HA filled in PDMS devices}

A $1 \mathrm{mg} / \mathrm{ml}$ fluorescein solution was prepared by dissolving fluorescein sodium salt in 1\% HA carrier liquid. Further, $10 \mathrm{mg} / \mathrm{ml}$ solutions of Blue Dextran ( 2 MDa) and FITC-labeled HA (1.26 MDa) were prepared by dissolving appropriate amounts of the compounds in PBS; the FITC-HA solution was gently heated $\left(40^{\circ} \mathrm{C}\right)$ and stirred for 16 hours to dissolve the polymer. The obtained solutions of Fluorescein, Blue Dextran and FITC-HA solutions were filled into PDMS devices as described in section Filling of PDMS devices. Filled devices were transferred into a glass vial containing $10 \mathrm{ml}$ of in vitro release buffer (IVR buffer; consisting of Dulbecco's PBS pH 7.4 (0.2 $\mu \mathrm{m}$ filtered), 0.025\% Tween 20, 0.02\% $\mathrm{NaN}_{3}$ ) and were then incubated at $37^{\circ} \mathrm{C}$ under mild agitation. At appropriate time points, a $1 \mathrm{ml}$ sample of the release medium was removed and replaced with fresh release buffer. The fluorescein or FITC-HA content in release samples was quantified by spectrofluorometry at $\lambda_{\text {ex: }}: 493 \mathrm{~nm}$ and $\lambda_{\mathrm{em}}: 512 \mathrm{~nm}$ using a Jasco spectrofluorometer FP-8300 (Jasco, Easton, MD, USA). The Blue Dextran content in release samples was determined by UV spectroscopy at $620 \mathrm{~nm}$ using a Spectrostar Nano plate reader (BMG Labtech, Ortenberg, Germany).

\section{Rheological measurements of microsphere dispersion}

Microsphere dispersions of 6, 12,20,30 and $50 \mathrm{mg} / \mathrm{ml}$ were prepared essentially as described in section Preparation of microsphere dispersion in HA carrier liquid and analyzed for their rheological properties using a Discovery HR-2 rheometer (TA instruments, Etten-Leur, the Netherlands) equipped with a parallel plate measuring geometry (steel plate diameter: $40 \mathrm{~mm}$ ). A shear stress ramp was obtained from 0.01 to $1 \mathrm{~Pa}$ at $21^{\circ} \mathrm{C}$.

\section{Release of VEGF from microspheres filled in PDMS devices}

Microsphere dispersions were prepared by weighing the appropriate amount of microspheres and suspending them in HA carrier liquid (final concentration $12 \mathrm{mg} / \mathrm{ml}$, see section Preparation of microsphere dispersion in HA carrier liquid) and filled into PDMS devices as described in section Filling of PDMS devices. PDMS devices filled with VEGF-loaded microspheres were transferred into a glass vial containing $10 \mathrm{ml}$ of in vitro release buffer (IVR buffer; consisting of Dulbecco's PBS pH 7.4 (0.2 $\mu \mathrm{m}$ filtered), 0.025\% Tween 20,0.02\% $\mathrm{NaN}_{3}$ ) and were then 
incubated at $37^{\circ} \mathrm{C}$ under mild agitation. At appropriate time points, the complete release medium was removed and replaced with fresh IVR buffer. Released VEGF was quantified by a sandwich enzyme-linked immunosorbent assay (ELISA) (Human VEGF DuoSet ELISA, R\&D Systems, Abingdon, United Kingdom) according to the manufacturer's protocol. The VEGF stock solution of the ELISA kit was used for calibration in the concentration range of 31 - 2000 $\mathrm{pg} / \mathrm{ml}$. Release samples were diluted with reagent diluent to fall within the working range of the assay and measured in duplicate. ELISA plates were read at $450 \mathrm{~nm}$ using a Spectrostar Nano plate reader (BMG Labtech, Ortenberg, Germany).

Obtained VEGF release curves were fitted using the Korsmeyer-Peppas model, where $Q_{t}$ is the amount of drug released from microspheres at time point $t, Q_{0}$ is the initial amount of drug in microspheres, $n$ is diffusional exponent indicative of the transport mechanism, and $K_{p}$ is Korsmeyer-Peppas constant incorporating structural and geometric characteristics of the dosage form (equation 1). ${ }^{42}$ The diffusional exponent $n$ was calculated from the fitted linear regression lines of log (\% drug released) versus log (time) (equation 2). The obtained parameters of fitted VEGF release from microspheres filled in PDMS devices, were compared to the parameters of the fit of the VEGF release curves from microspheres in IVR buffer, which have been reported previously. ${ }^{35}$

$$
\frac{Q_{t}}{Q_{0}}=K_{p} \times t^{n}
$$

EQUATION 1: Korsmeyer-Peppas equation

$$
\log \left(\frac{Q_{t}}{Q_{0}}\right)=\log \left(K_{p}\right)+n \log (t)
$$

EQUATION 2: adapted Korsmeyer-Peppas equation

\section{Bioactivity of VEGF released from PDMS devices}

The bioactivity of released VEGF from the implants was analyzed by Alamar Blue proliferation assay with human umbilical vein endothelial cells (HUVECS), as described previously. ${ }^{35}$ In short, a $12 \mathrm{mg} / \mathrm{ml}$ microsphere dispersion of VEGF-loaded microspheres in 1\% HA carrier liquid was prepared as described in section Release of VEGF from microspheres filled in PDMS devices and filled in PDMS devices (section Filling of PDMS devices). Filled PDMS devices were incubated in $10 \mathrm{ml}$ cell-compatible IVR buffer ("bioactivity IVR buffer"; Dulbecco's PBS pH 7.4, 0.2 um filtered, $0.5 \%$ BSA, $30 \mu \mathrm{g} / \mathrm{ml}$ gentamicin and $15 \mathrm{ng} / \mathrm{ml}$ amphotericin) at $37{ }^{\circ} \mathrm{C}$ under mild agitation. After 1, 2, 3 and 4 weeks of incubation, the complete release medium was removed and replaced by fresh bioactivity IVR buffer. Release samples were diluted in bioactivity medium to fall within the (linear) proliferation range of VEGF (0 to $20 \mathrm{ng} / \mathrm{ml}$ ), corresponding to a relative cell proliferation of 1 - 3.5 (defined by the proliferation in \% normalized by the proliferation of cells that were incubated without VEGF). 


\section{Statistical analysis}

Data are presented as average with SD. Statistical analysis was performed with GraphPad Prism7 using the one-way analysis of variance (ANOVA) and Holm-Sidak multicomparison test. Differences between the analyzed groups were considered significant if $p<0.05$.

\section{Results}

\section{Characterization of PDMS devices}

Figure 1a shows a schematic image of the PDMS devices used in this study. PDMS devices were made by a 3-step printing process. First, two separately prepared membrane sheets were made from a PDMS/porogen mix which was subsequently crosslinked. Next, connecting support structures (PDMS, no porogen) were printed on the outline and inner parts of one membrane (as shown in figure $1 \mathrm{~b}$ in white). Then, a second membrane was placed on top of the support structures and the polymer was again crosslinked. Finally, reinforcement structures (PDMS) were printed on the two outer surfaces of the membranes and subsequently crosslinked. The dimensions of the printed devices were chosen for future use in rodent studies, aiming for subcutaneous implantation of the devices. Typically, the devices measured $30 \times 14 \times 0.8 \mathrm{~mm}$, with an internal volume of $\sim 160 \mu$ lactive inner area of $20 \times 10 \times 0.8$ $\mathrm{mm}$ ). The surface morphology of the biomaterial implants was created by 3D-printing of a reinforcement structure of PDMS. Pores were introduced into the membranes by adding $\mathrm{NaCl}$ crystals $(7-8 \mu \mathrm{m})$ as porogen to PDMS which were washed out with water after the printing process had been completed. Macroscopic pictures of the devices are shown in figure $1 \mathrm{~b}$ and 1c, whereby the distribution of microspheres within the inner lumen of the implants is visualized with Nile Red-labelled microspheres (as shown in reddish-pink color). The microspheres were distributed homogeneously in the accessible parts of the implants while support structures were not colored. The morphology of the 3D printed PDMS was analyzed by scanning electron microscopy (figure $1 \mathrm{~d}$-e). Both surface and cross-sections of the device were selected. The reinforcement structures on the outside of the implants were smooth and $\sim 300 \mu \mathrm{m}$ thick (figure 1d). The outer surface of the membrane was smooth with a few pores ranging between $\sim 4$ and $10 \mu \mathrm{m}$ ) visible (figure e). As seen in the cross-sections (figure $1 \mathrm{f}$ and $\mathrm{g}$ ), the inside of the implant was up to $200 \mu \mathrm{m}$ wide (figure $1 \mathrm{f}$ ) and the membrane sheet had a porous network (pore sizes between 4 - $20 \mu \mathrm{m}$; figure 1g). The release of possible toxic leachables was studied with devices filled with HA carrier liquid. Release supernatants were collected in weekly intervals up to four weeks of incubation, and did not show sign of toxic effects (viability of HUVEC remained above 95\% for all conditions), as shown in figure S2. Devices were filled with a fluorescein sodium solution in HA carrier liquid, to evaluate whether small molecules can permeate through the PDMS membrane. As shown in figure 2 (yellow release curve), fluorescein was released completely within 2 hours. In a similar setup, 
we evaluated the efflux of Blue Dextran (molecular weight of $2 \mathrm{MDa}$; blue release curve) and FITC-labelled HA (molecular weight of 1.26 MDa; orange release curve) from PDMS devices. Both polymers were released from the devices within 48 hours with almost similar release kinetics.
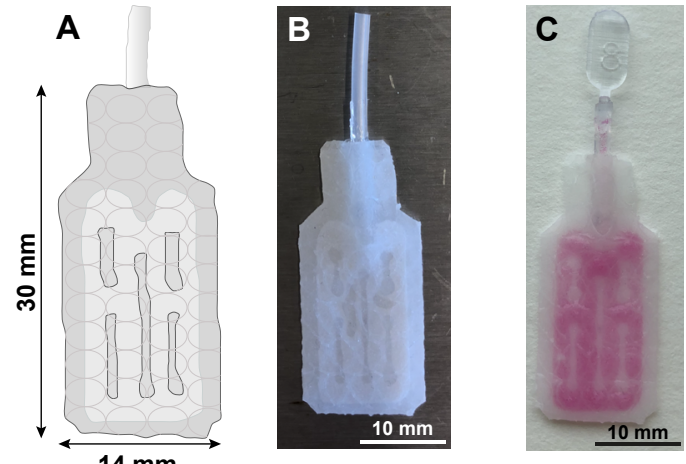

\section{DEVICE SURFACE}
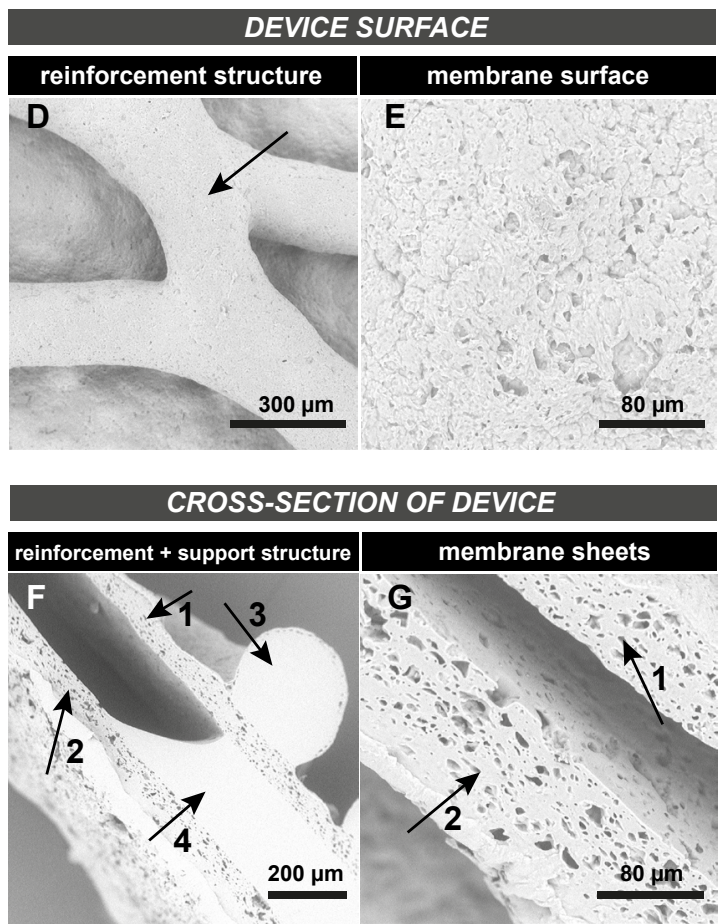

FIGURE 1: Imaging of PDMS devices (30 mm x $14 \mathrm{~mm}$ ): A: Schematic image of a PDMS device. B: Photograph of a dry device with plastic inlet. C: Photograph of a wetted device, filled with Nile Red-labelled microspheres (in pink). $D$ and E: Scanning electron microscopy (SEM) images of the device surface, D: reinforcement structure on the outer surface of the device (indicated with arrow), E: membrane morphology of the outer surface of the device. F and G: cross-section of device. F: two membrane sheets (arrow 1 and 2), outer reinforcement structure (arrow 3), support structure between two membrane sheets (arrow 4). G: close-up of two porous membrane sheets (arrows 1 and 2). 


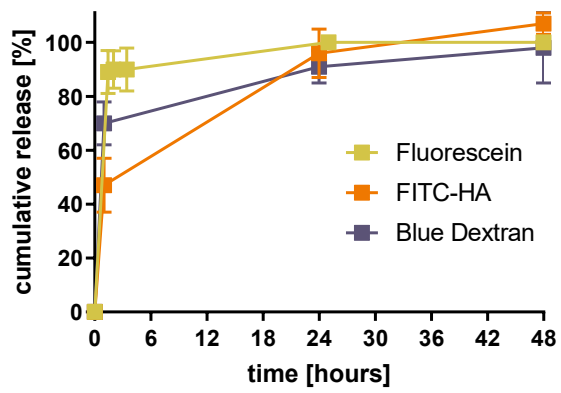

FIGURE 2: Cumulative release of fluorescein, FITC-HA and Blue Dextran from PDMS devices. The devices were filled with a solution of fluorescein in HA carrier liquid, FITC-labelled HA (FITC-HA) or Blue Dextran in PBS, and were subsequently incubated at $37^{\circ} \mathrm{C}$ in IVR buffer.

\section{Viscosity of microsphere dispersions in HA carrier liquid}

In view of the final aim of loading pancreatic islets into the devices, it is crucial that dispersions of microspheres in HA carrier liquid can be injected into the PDMS devices via a 18G needle with only minimal injection force. We therefore investigated the rheological behavior of microsphere dispersions in HA carrier liquid with concentrations ranging from $6-50 \mathrm{mg} /$ $\mathrm{ml}$. As shown in figure 3 (gray circles), 1\% HA carrier liquid displayed a constant viscosity of $0.5 \mathrm{~Pa}^{*} \mathrm{~s}$ at stresses ranging from 0.01 to $1.0 \mathrm{~Pa}$, showing Newtonian rheological behavior (meaning that the viscosity is independent of the applied shear stress). On the other hand, dispersions of microspheres in HA carrier liquid showed shear-thinning behavior. The viscosity of dispersions of $6-20 \mathrm{mg} / \mathrm{ml}$ ranged from $1.0-1.7 \mathrm{~Pa}^{*} \mathrm{~s}$ and decreased to about $0.5 \mathrm{~Pa}^{*} \mathrm{~s}$ at shear stresses above $0.05 \mathrm{~Pa}$. The microsphere dispersion of $30 \mathrm{mg} / \mathrm{ml}$ showed a decrease in viscosity from $\sim 7.0 \mathrm{~Pa}^{*} \mathrm{~s}$ to $0.5 \mathrm{~Pa}$ at shear stresses above $0.40 \mathrm{~Pa}$. Similarly, the viscosity of 50 $\mathrm{mg} / \mathrm{ml}$ microsphere dispersion decreased from $\sim 9.0 \mathrm{~Pa}^{*} \mathrm{~s}$ to $\sim 1.0 \mathrm{~Pa}^{*} \mathrm{~s}$ at shear stresses above $0.60 \mathrm{~Pa}$. The yield stress, defined as the shear stress at which the microsphere dispersion starts to flow, ${ }^{43}$ of dispersions at the tested concentrations $(6-50 \mathrm{mg} / \mathrm{ml})$ was relatively low, i.e. < 0.03 Pa for $6-20 \mathrm{mg} / \mathrm{ml}$ and $\sim 0.15$ Pa for 30 and $50 \mathrm{mg} / \mathrm{ml}$, comparable to those of orange juice or blue ink. ${ }^{b}$ We therefore conclude that all microsphere dispersions are easily injectable through an $18 \mathrm{G}$ needle. 


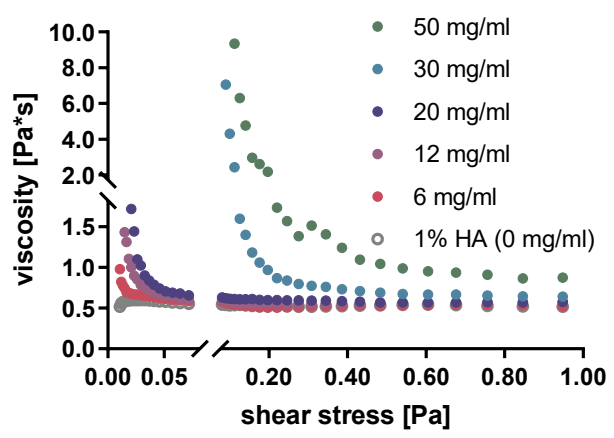

FIGURE 3: Rheological measurements of microsphere dispersions in 1\% HA carrier liquid (concentrations of microspheres stated in $\mathrm{mg} / \mathrm{ml}$ ). Viscosities (y-axis) were measured using a shear stress ramp from 0.01-1.0 Pa ( $\mathrm{x}$-axis) at $21^{\circ} \mathrm{C}$.

\section{Incorporation of VEGF microspheres in devices and VEGF release}

We selected $12 \mathrm{mg} / \mathrm{ml}$ as the preferred concentration of microspheres since at the loaded VEGF concentration and the inner volume of the devices, this would represent an appropriate theoretical dose of VEGF (i.e. 150 ng loaded VEGF, yielding an expected VEGF release of $5 \mathrm{ng} /$ day, assuming continuous release of the loaded dose over 28 days ${ }^{35}$ ). Figure 4 shows that VEGF was released from PDMS devices throughout the release period 28 days in a similar pattern as VEGF release measured from microspheres in suspension as published previously. ${ }^{35}$ Indeed, the diffusional exponent for microspheres filled in devices ( $0.43 \pm 0.03$ ) was comparable to the diffusional exponent of $0.41 \pm 0.02$ for microspheres in suspension, as derived from nonexponential fitting by the Korsmeyer-Peppas model (table 1).42,44

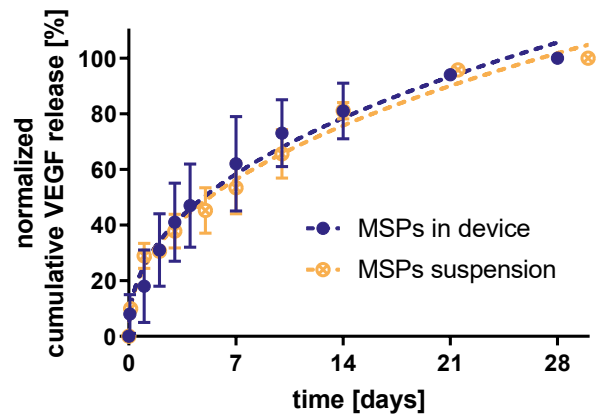

FIGURE 4: Normalized cumulative release [\%] of VEGF from PDMS devices filled with VEGF-loaded microspheres (blue curve) and VEGF-loaded microspheres in suspension (orange curve, adapted from Scheiner et al., ACS Omega ${ }^{35}$ ). Release experiments were performed in in vitro release (IVR) buffer at $37^{\circ} \mathrm{C}(\mathrm{n}=3$, shown as average \pm standard deviation). Released VEGF was quantified by ELISA and normalized to $100 \%$ based on the total release observed under the test conditions. 
TABLE 1: Korsmeyer-Peppas model fit parameters for normalized cumulative VEGF release curves measured by ELISA of microspheres in suspension and incorporated in PDMS devices, as shown in figure 4 as orange and blue dotted lines, respectively.

\begin{tabular}{ccc}
\hline \multirow{2}{*}{ Parameters $^{\mathbf{a}}$} & \multicolumn{2}{c}{ VEGF release quantified by ELISA } \\
\cline { 2 - 3 } & MSP suspension & MSPs in device \\
\hline$n$ & $0.41 \pm 0.02$ & $0.43 \pm 0.03$ \\
$95 \% \mathrm{Cl}$ & $0.37-0.44$ & $0.37-0.49$ \\
$R^{2}$ & 0.97 & 0.91 \\
\hline
\end{tabular}

${ }^{a}$ : diffusional exponent, $95 \% \mathrm{Cl}$ : 95\% confidence interval, $R^{2}$ : correlation coefficient.

\section{Bioactivity of VEGF released from PDMS devices}

The bioactivity of VEGF released from the PDMS devices was assessed with a HUVEC proliferation assay. As shown in figure 5, endothelial cell proliferation was stimulated up to 3-fold by spiked VEGF samples in the dose range of 0.5 - $20 \mathrm{ng} / \mathrm{ml}$ (see figure S3 for a detailed calibration curve of VEGF). In vitro release (IVR) samples of all time points, diluted 20 or 100x, stimulated endothelial cell proliferation between 1.5 - 3-fold in a dose-dependent manner corresponding to the expected concentrations of $2-10 \mathrm{ng} / \mathrm{ml}$ (for 100-fold and 20-fold diluted samples, respectively). Importantly, figure 5 confirms that bioactive VEGF was released during the full four-week timespan of the release study. Control experiments with PDMS devices filled with placebo microspheres did not stimulate endothelial cell proliferation.

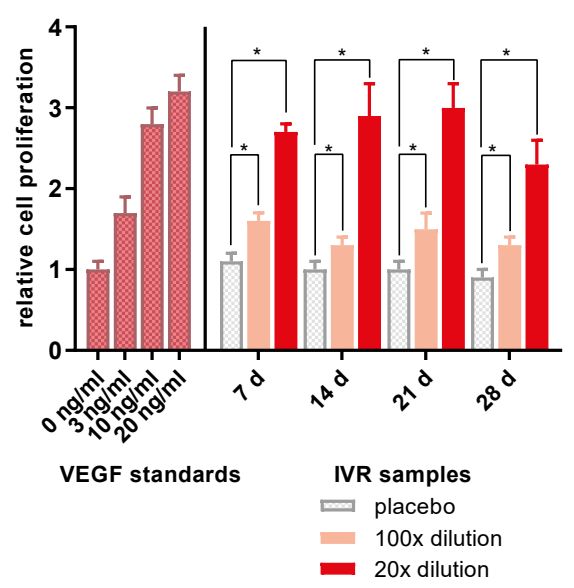

FIGURE 5: Bioactivity of VEGF released from PDMS devices filled with VEGF-loaded microspheres. On the left side of the graph (pink bars: linear responsive range of VEGF standards between 0 and $20 \mathrm{ng} / \mathrm{ml}$. On the right side of the graph (gray, light red and red bars): relative cell proliferation of released VEGF ("IVR samples") from PDMS devices filled with VEGF-loaded microspheres per time point (d: days). IVR supernatants were diluted 100x (light red bars) and 20x (red bars). IVR supernatants of devices filled with placebo microspheres served as controls (gray bars; "placebo). The release study was performed in cell-compatible "bioactivity IVR buffer" without sodium azide and Tween 20 at $37{ }^{\circ} \mathrm{C}$. Bars represent average $\pm \mathrm{SD}$ of $\mathrm{n}=3 .{ }^{*} p<0.05100 x$ dilution or $20 x$ dilution versus placebo IVR samples. 


\section{Discussion}

The aim of this study was to develop an in vitro prevascularization strategy of PDMS devices by incorporating microspheres that release vascular endothelial growth factor (VEGF), the most prominent proangiogenic growth factor. ${ }^{45}$ These microspheres have demonstrated continuous VEGF release for four weeks, ${ }^{35}$ which has been showed to be a favorable release window for implant vascularization. ${ }^{46,47}$ Devices used in this study were prepared with PDMS, a polymer that has been well-studied for its application for pancreatic islet transplantation due to its bioinertia and biocompatibility. ${ }^{38,48,49}$ SEM revealed a porous network within the PDMS membrane which is needed for efficient in and out diffusion of nutrients and proteins. ${ }^{50,}$ ${ }^{51}$ Indeed, the permeability of the membrane was demonstrated by complete release of fluorescein, FITC-labelled HA and Blue Dextran from PDMS implant within 48 hours. Hence, we do not expect a significant barrier function for nutrients and other small molecules that need to diffuse from biological fluids into and from the device upon transplantation. Furthermore, our results show biocompatibility of PDMS devices, in line with previous studies. ${ }^{52,53}$ The rheological measurements of microsphere dispersions with concentrations ranging from 6 - $50 \mathrm{mg} / \mathrm{ml}$ revealed low yield pressures and thus excellent syringeability for filling in PDMS devices. VEGF-loaded microspheres filled in PDMS devices showed sustained release of this protein for 4 weeks. Figure 4 shows that the devices had the same release characteristics as the microspheres suspended in buffer, i.e. diffusion-controlled release (see table 1) through a water-filled porous network within the polymeric matrix of the microspheres, caused by the hydrophilic PEG of the multiblock copolymers..$^{35}$ This means that VEGF release from the devices is neither controlled by diffusion over the PDMS membrane nor binding to PDMS, and it is primarily controlled by its release from the microspheres. This is not surprising as the diameters of membrane pores (as visible by SEM, figure 1) created by salt leaching are much bigger than the hydrodynamic radius of VEGF and Blue Dextran $\left(R_{H} \sim 27 \mathrm{~nm}^{54}\right)$. Importantly, VEGF was released in its bioactive form from microspheres loaded in PDMS devices. The released amount, based on the chosen microsphere concentration $(12 \mathrm{mg} / \mathrm{ml})$ and release of bioactive VEGF (figure 5), is considered optimal for sufficient vascularization and thus performance of biomaterial implants, i.e. $150 \mathrm{ng} / 0.1 \mathrm{ml}$ implant for four weeks. ${ }^{15,25}$ Although it is known that VEGF is well suitable for vascularization of biomaterial implants, ${ }^{11-13}$ angiogenesis in vivo is an interplay of several different growth factors and cytokines, such as fibroblast growth factor (FGF), hepatocyte growth factor (HGF) and platelet-derived growth factor (PDGF), besides VEGF.16,55 Polymeric microspheres offer the possibility to encapsulate and release other growth factors as well, and these microspheres can also be incorporated in biomaterial devices intended for implantation in vivo, such as PDMS devices used in this study.

In future in vivo studies, we aim to investigate the suitability for this device design for pancreatic islet transplantation in a rodent diabetes model. Two options are possible for integrating pancreatic islets with the vascularization approach of this study. Our current work has focused 
on developing a protocol for mixing rat pancreatic islets with VEGF-loaded microspheres in a HA-based suspension and subsequently filling this suspension in PDMS devices. Besides this, a prevascularization approach also seems feasible, where PDMS devices are first filled with VEGF-loaded microsphere suspension and implanted subcutaneously in rats. After 4 weeks, pancreatic islets can then be added into the (vascularized) PDMS device via the inlet. For both options, the compatibility of pancreatic islets with VEGF and VEGF-loaded microspheres needs to be ensured and is therefore part of our current investigations.

\section{Conclusions}

In this study, we have incorporated VEGF-releasing microspheres in PDMS-based devices using 1\% HA solution as carrier liquid. PDMS devices, created with salt leaching and 3D-printing, showed a porous network and high permeability of fluorescein, FITC-labelled HA and Blue Dextran. PDMS devices filled with HA carrier liquid showed cytocompatibility with endothelial cells. Microsphere dispersions of various concentrations in HA carrier liquid showed good syringeability. VEGF was released continuously from PDMS devices for four weeks, with release kinetics comparable to microspheres in suspension in buffer. The release of VEGF from PDMS devices is therefore governed by the microspheres, as no significant delay by the PDMS membrane was observed. Released VEGF from PDMS devices remained bioactive over the entire release period of four weeks. In conclusion, PDMS devices functionalized with VEGFreleasing microspheres are an attractive vascularization strategy for pancreatic islet-filled implants, which will be studied in future in vivo studies.

\section{Acknowledgements}

This work was supported by European Union's Horizon 2020 research and innovation program Diabetes-reversing implants for enhanced viability and long-term efficacy (DRIVE) [grant agreement number 645991]. The authors would like to thank Marcel Fens, Cedric Hustinx, Johanna Walther, Joep van den Dikkenberg and Mies van Steenbergen for their excellent practical assistance. Contipro (Prague, Czech Republic) is acknowledged for providing hyaluronic acid. 


\section{References}

1. Richardson, S. J.; Morgan, N. G.; Foulis, A. K. Pancreatic pathology in type 1 diabetes mellitus. Endocr. Pathol. 2014, 25, (1), 80-92.

2. Shapiro, A. M.; Pokrywczynska, M.; Ricordi, C. Clinical pancreatic islet transplantation. Nat. Rev. Endocrinol. 2017, 13, (5), 268-277.

3. Khosravi-Maharlooei, M.; Hajizadeh-Saffar, E.; Tahamtani, Y.; Basiri, M.; Montazeri, L.; Khalooghi, K.; Kazemi Ashtiani, M.; Farrokhi, A.; Aghdami, N.; Sadr Hashemi Nejad, A.; Larijani, M. B.; De Leu, N.; Heimberg, H.; Luo, X.; Baharvand, H. Islet transplantation for type 1 diabetes: so close and yet so far away. Eur. J. Endocrinol. 2015, 173, (5), R165-83.

4. Shapiro, A. M.; Lakey, J. R.; Ryan, E. A.; Korbutt, G. S.; Toth, E.; Warnock, G. L.; Kneteman, N. M.; Rajotte, R. V. Islet transplantation in seven patients with type 1 diabetes mellitus using a glucocorticoid-free immunosuppressive regimen. N. Engl. J. Med. 2000, 343, (4), 230-8.

5. Desai, T.; Shea, L. D. Advances in islet encapsulation technologies. Nat. Rev. Drug Discov. 2017, 16, (5), $338-350$.

6. Canibano-Hernandez, A.; Saenz Del Burgo, L.; Espona-Noguera, A.; Ciriza, J.; Pedraz, J. L. Current advanced therapy cell-based medicinal products for type-1-diabetes treatment. Int. J. Pharm. 2018, 543, (1-2), 107-120.

7. Dimitrioglou, N.; Kanelli, M.; Papageorgiou, E.; Karatzas, T.; Hatziavramidis, D. Paving the way for successful islet encapsulation. Drug Discov. Today 2019, 24, (3), 737-748.

8. Uematsu, S. S.; Inagaki, A.; Nakamura, Y.; Imura, T.; Igarashi, Y.; Fathi, I.; Miyagi, S.; Ohuchi, N.; Satomi, S.; Goto, M. The optimization of the prevascularization procedures for improving subcutaneous islet engraftment. Transplantation 2018, 102, (3), 387-395.

9. Farina, M.; Chua, C. Y. X.; Ballerini, A.; Thekkedath, U.; Alexander, J. F.; Rhudy, J. R.; Torchio, G.; Fraga, D.; Pathak, R. R.; Villanueva, M.; Shin, C. S.; Niles, J. A.; Sesana, R.; Demarchi, D.; Sikora, A. G.; Acharya, G. S.; Gaber, A. O.; Nichols, J. E.; Grattoni, A. Transcutaneously refillable, 3D-printed biopolymeric encapsulation system for the transplantation of endocrine cells. Biomaterials 2018, 177, 125-138.

10. Smink, A. M.; Li, S.; Hertsig, D. T.; de Haan, B. J.; Schwab, L.; van Apeldoorn, A. A.; de Koning, E.; Faas, M. M.; Lakey, J. R.; de Vos, P. The efficacy of a prevascularized, retrievable poly(D,L,-lactide-co-epsilon-caprolactone) subcutaneous scaffold as transplantation site for pancreatic islets. Transplantation 2017, 101, (4), e112-e119.

11. Wagner, E. R.; Parry, J.; Dadsetan, M.; Bravo, D.; Riester, S. M.; Van Wijnen, A. J.; Yaszemski, M. J.; Kakar, S. VEGFmediated angiogenesis and vascularization of a fumarate-crosslinked polycaprolactone (PCLF) scaffold. Connect. Tissue Res. 2018, 59, (6), 542-549.

12. Quinlan, E.; Lopez-Noriega, A.; Thompson, E. M.; Hibbitts, A.; Cryan, S. A.; O'Brien, F. J. Controlled release of vascular endothelial growth factor from spray-dried alginate microparticles in collagen-hydroxyapatite scaffolds for promoting vascularization and bone repair. J. Tissue Eng. Regen. Med. 2017, 11, (4), 1097-1109.

13. Farina, M.; Ballerini, A.; Fraga, D. W.; Nicolov, E.; Hogan, M.; Demarchi, D.; Scaglione, F.; Sabek, O. M.; Horner, P.; Thekkedath, U.; Gaber, O. A.; Grattoni, A. 3D printed vascularized device for subcutaneous transplantation of human islets. Biotechnol J 2017, 12, (9).

14. Silva, E. A.; Mooney, D. J. Effects of VEGF temporal and spatial presentation on angiogenesis. Biomaterials 2010, $31,(6), 1235-41$.

15. Davies, N.; Dobner, S.; Bezuidenhout, D.; Schmidt, C.; Beck, M.; Zisch, A. H.; Zilla, P. The dosage dependence of VEGF stimulation on scaffold neovascularisation. Biomaterials 2008, 29, (26), 3531-8.

16. Said, S. S.; Pickering, J. G.; Mequanint, K. Advances in growth factor delivery for therapeutic angiogenesis. J. Vasc. Res. 2013, 50, (1), 35-51.

17. Lee, K.; Silva, E. A.; Mooney, D. J. Growth factor delivery-based tissue engineering: general approaches and a review of recent developments. J $R$ Soc Interface 2011, 8, (55), 153-70.

18. Giteau, A.; Venier-Julienne, M. C.; Aubert-Pouessel, A.; Benoit, J. P. How to achieve sustained and complete protein release from PLGA-based microparticles? Int. J. Pharm. 2008, 350, (1-2), 14-26.

19. Ma, G. Microencapsulation of protein drugs for drug delivery: strategy, preparation, and applications. J. Control. Release 2014, 193, 324-40. 
20. Rui, J.; Dadsetan, M.; Runge, M. B.; Spinner, R. J.; Yaszemski, M. J.; Windebank, A. J.; Wang, H. Controlled release of vascular endothelial growth factor using poly-lactic-co-glycolic acid microspheres: in vitro characterization and application in polycaprolactone fumarate nerve conduits. Acta Biomater. 2012, 8, (2), 511-8.

21. van de Weert, M.; Hennink, W. E.; Jiskoot, W. Protein instability in poly(lactic-co-glycolic acid) microparticles. Pharm. Res. 2000, 17, (10), 1159-67.

22. Schwendeman, S. P. Recent advances in the stabilization of proteins encapsulated in injectable PLGA delivery systems. Crit. Rev. Ther. Drug Carrier Syst. 2002, 19, (1), 73-98.

23. Simon-Yarza, T.; Tamayo, E.; Benavides, C.; Lana, H.; Formiga, F. R.; Grama, C. N.; Ortiz-de-Solorzano, C.; Kumar, M. N.; Prosper, F.; Blanco-Prieto, M. J. Functional benefits of PLGA particulates carrying VEGF and CoQ10 in an animal of myocardial ischemia. Int. J. Pharm. 2013, 454, (2), 784-90.

24. Simon-Yarza, T.; Formiga, F. R.; Tamayo, E.; Pelacho, B.; Prosper, F.; Blanco-Prieto, M. J. PEGylated-PLGA microparticles containing VEGF for long term drug delivery. Int. J. Pharm. 2013, 440, (1), 13-8.

25. Amsden, B. G.; Timbart, L.; Marecak, D.; Chapanian, R.; Tse, M. Y.; Pang, S. C. VEGF-induced angiogenesis following localized delivery via injectable, low viscosity poly(trimethylene carbonate). J. Control. Release 2010, 145, (2), 109-15.

26. Fu, K.; Pack, D. W.; Klibanov, A. M.; Langer, R. Visual evidence of acidic environment within degrading poly(lacticco-glycolic acid) (PLGA) microspheres. Pharm. Res. 2000, 17, (1), 100-6.

27. Shenderova, A.; Burke, T. G.; Schwendeman, S. P. The acidic microclimate in poly(lactide-co-glycolide) microspheres stabilizes camptothecins. Pharm. Res. 1999, 16, (2), 241-8.

28. Giteau, A.; Venier-Julienne, M. C.; Marchal, S.; Courthaudon, J. L.; Sergent, M.; Montero-Menei, C.; Verdier, J. M.; Benoit, J. P. Reversible protein precipitation to ensure stability during encapsulation within PLGA microspheres. Eur. J. Pharm. Biopharm. 2008, 70, (1), 127-36.

29. Estey, T.; Kang, J.; Schwendeman, S. P.; Carpenter, J. F. BSA degradation under acidic conditions: a model for protein instability during release from PLGA delivery systems. J. Pharm. Sci. 2006, 95, (7), 1626-39.

30. Kissel, T.; Li, Y.; Unger, F. ABA-triblock copolymers from biodegradable polyester A-blocks and hydrophilic poly(ethylene oxide) B-blocks as a candidate for in situ forming hydrogel delivery systems for proteins. Adv Drug Deliv Rev 2002, 54, (1), 99-134.

31. Bonacucina, G.; Cespi, M.; Mencarelli, G.; Giorgioni, G.; Palmieri, G. F. Thermosensitive self-assembling block copolymers as drug delivery systems. Polymers-Basel 2011, 3, (2), 779-811.

32. Stankovic, M.; Hiemstra, C.; de Waard, H.; Zuidema, J.; Steendam, R.; Frijlink, H. W.; Hinrichs, W. L. Protein release from water-swellable poly(D,L-lactide-PEG)-b-poly(-caprolactone) implants. Int. J. Pharm. 2015, 480, (1-2), 7383.

33. Stankovic, M.; Tomar, J.; Hiemstra, C.; Steendam, R.; Frijlink, H. W.; Hinrichs, W. L. Tailored protein release from biodegradable poly(epsilon-caprolactone-PEG)-b-poly(epsilon-caprolactone) multiblock-copolymer implants. Eur. J. Pharm. Biopharm. 2014, 87, (2), 329-37.

34. Teekamp, N.; Van Dijk, F.; Broesder, A.; Evers, M.; Zuidema, J.; Steendam, R.; Post, E.; Hillebrands, J. L.; Frijlink, H. W.; Poelstra, K.; Beljaars, L.; Olinga, P.; Hinrichs, W. L. J. Polymeric microspheres for the sustained release of a protein-based drug carrier targeting the PDGFbeta-receptor in the fibrotic kidney. Int. J. Pharm. 2017, 534, (1-2), 229-236.

35. Scheiner, K. C.; Maas-Bakker, R. F.; Nguyen, T. T.; Duarte, A. M.; Hendriks, G.; Sequeira, L.; Duffy, G. P.; Steendam, R.; Hennink, W. E.; Kok, R. J. Sustained release of vascular endothelial growth factor from poly(epsiloncaprolactone-PEG-epsilon-caprolactone)-b-poly(l-lactide) multiblock copolymer microspheres. ACS Omega 2019, 4, (7), 11481-11492.

36. Sandker, M. J.; Duque, L. F.; Redout, E. M.; Chan, A.; Que, I.; Lowik, C. W.; Klijnstra, E. C.; Kops, N.; Steendam, R.; van Weeren, R.; Hennink, W. E.; Weinans, H. Degradation, intra-articular retention and biocompatibility of monospheres composed of [PDLLA-PEG-PDLLA]-b-PLLA multi-block copolymers. Acta Biomater. 2017, 48, 401414.

37. Harrington, S.; Williams, J.; Rawal, S.; Ramachandran, K.; Stehno-Bittel, L. Hyaluronic acid/collagen hydrogel as an alternative to alginate for long-term immunoprotected islet transplantation. Tissue Eng Part A 2017, 23, (1920), 1088-1099. 
38. Pedraza, E.; Brady, A. C.; Fraker, C. A.; Molano, R. D.; Sukert, S.; Berman, D. M.; Kenyon, N. S.; Pileggi, A.; Ricordi, C.; Stabler, C. L. Macroporous three-dimensional PDMS scaffolds for extrahepatic islet transplantation. Cell Transplant. 2013, 22, (7), 1123-35.

39. Shrestha, P.; Regmi, S.; Jeong, J.-H. Injectable hydrogels for islet transplantation: a concise review. Journal of Pharmaceutical Investigation 2019.

40. Chia, H. N.; Wu, B. M. Recent advances in 3D printing of biomaterials. J. Biol. Eng. 2015, 9, 4.

41. Coulter, F. B.; O'Cearbhaill, E. D., A PDMS-based 3D-printed biomaterial implant for subcutaneous transplantation of pancreatic islets. [unpublished results]

42. Ritger, P. L.; Peppas, N. A. A simple equation for description of solute release II. Fickian and anomalous release from swellable devices. J. Control. Release 1987, 5, 37 - 42.

43. Mouser, V. H.; Melchels, F. P.; Visser, J.; Dhert, W. J.; Gawlitta, D.; Malda, J. Yield stress determines bioprintability of hydrogels based on gelatin-methacryloyl and gellan gum for cartilage bioprinting. Biofabrication 2016, 8, (3), 035003

44. Costa, P.; Sousa Lobo, J. M. Modeling and comparison of dissolution profiles. Eur. J. Pharm. Sci. 2001, 13, (2), 123-33.

45. Yla-Herttuala, S.; Rissanen, T. T.; Vajanto, l.; Hartikainen, J. Vascular endothelial growth factors: biology and current status of clinical applications in cardiovascular medicine. J. Am. Coll. Cardiol. 2007, 49, (10), 1015-26.

46. Rouwkema, J.; Rivron, N. C.; van Blitterswijk, C. A. Vascularization in tissue engineering. Trends Biotechnol. 2008, 26, (8), 434-41.

47. Cao, L.; Mooney, D. J. Spatiotemporal control over growth factor signaling for therapeutic neovascularization. Adv Drug Deliv Rev 2007, 59, (13), 1340-50.

48. Abbasi, F.; Mirzadeh, H.; Katbab, A.-A. Modification of polysiloxane polymers for biomedical applications: a review. Polym. Int. 2001, 50, (12), 1279-1287.

49. Blanco, I. Polysiloxanes in theranostics and drug delivery: a review. Polymers (Basel) 2018, 10, (7).

50. Brauker, J. H.; Carr-Brendel, V. E.; Martinson, L. A.; Crudele, J; Johnston, W. D.; Johnson, R. C. Neovascularization of synthetic membranes directed by membrane microarchitecture. J. Biomed. Mater. Res. 1995, 29, (12), 1517-24.

51. Colton, C. K. Oxygen supply to encapsulated therapeutic cells. Adv Drug Deliv Rev 2014, 67-68, 93-110.

52. Laffleur, F.; Netsomboon, K.; Erman, L.; Partenhauser, A. Evaluation of modified hyaluronic acid in terms of rheology, enzymatic degradation and mucoadhesion. Int. J. Biol. Macromol. 2019, 123, 1204-1210.

53. Sivarapatna, A.; Ghaedi, M.; Xiao, Y.; Han, E.; Aryal, B.; Zhou, J.; Fernandez-Hernando, C.; Qyang, Y.; Hirschi, K. K.; Niklason, L. E. Engineered microvasculature in PDMS networks using endothelial cells derived from human induced pluripotent stem cells. Cell Transplant. 2017, 26, (8), 1365-1379.

54. Armstrong, J. K.; Wenby, R. B.; Meiselman, H. J.; Fisher, T. C. The hydrodynamic radii of macromolecules and their effect on red blood cell aggregation. Biophys. J. 2004, 87, (6), 4259-70.

55. Novosel, E. C.; Kleinhans, C.; Kluger, P. J. Vascularization is the key challenge in tissue engineering. Adv Drug Deliv $\operatorname{Rev}$ 2011, 63, (4-5), 300-11.

\section{Web references}

aEuropean Patent Office. European Patent Application EP 3409239A1 An implantable active agent encapsulating device. Available at: https://patentimages.storage.googleapis.com/f9/33/95/6517ae214da3fe/EP3409239A1.pdf. Accessed August 15, 2019.

'DTA instruments. Rheological Techniques for Yield Stress Analysis. Available at: https://www.tainstruments.com/pdf/ literature/RH025.pdf. Accessed August 15 th, 2019. 


\section{Supporting Information}

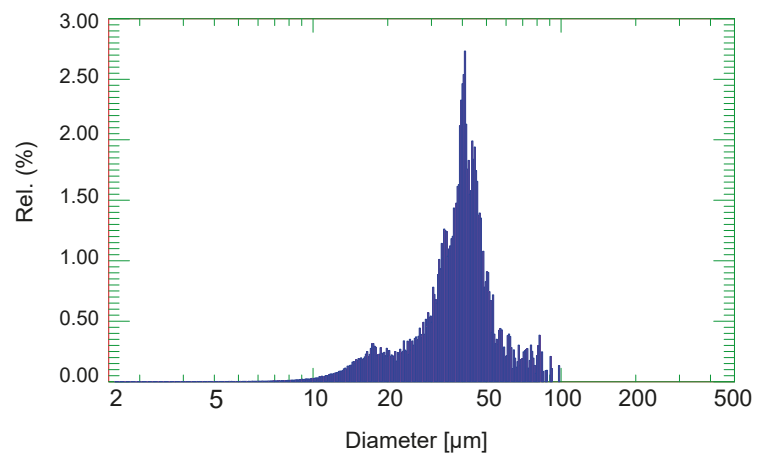

FIGURE S1: Size distribution of Nile Red-labelled PLGA microspheres prepared by membrane emulsification. Based on the volume-weight distribution, microspheres had a size of $39 \pm 14 \mu \mathrm{m}$.
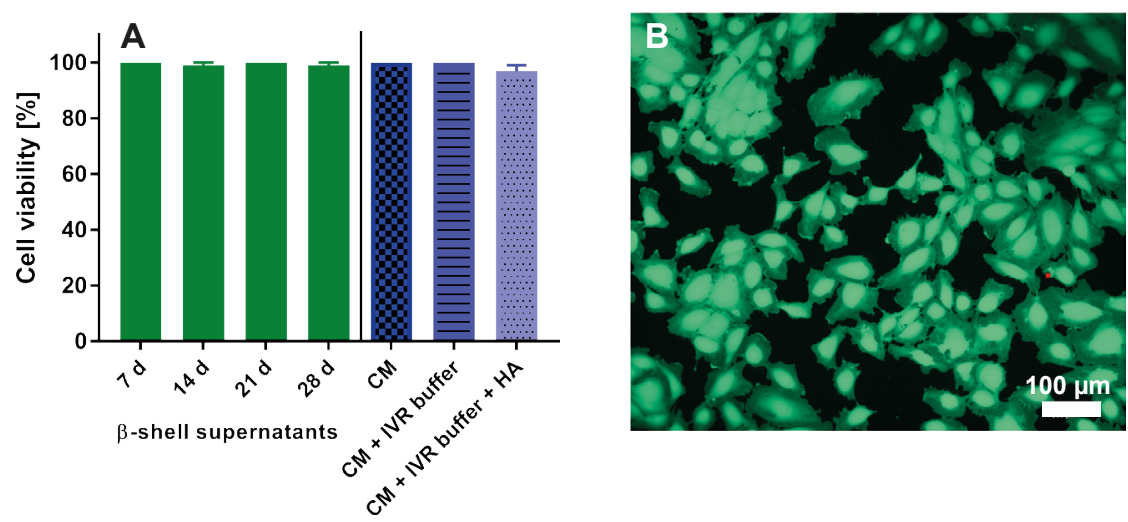

FIGURE S2: Live-dead cell staining of HUVEC incubated with IVR supernatants from placebo PDMS implants. A: Cell viability [\%] of endothelial cells quantified by live/dead staining (live: Calcein AM, dead: propidium iodide). Viability results were normalized versus cells incubated with control complete medium (CM). Control experiments included cells incubated with complete medium (CM), with cell-compatible IVR buffer diluted 20 times in complete medium (CM + IVR buffer), and with $8 \mu \mathrm{g} / \mathrm{ml} \mathrm{HA}$ in complete medium containing 20 times diluted cell-compatible IVR buffer $(\mathrm{CM}+$ IVR buffer $+\mathrm{HA})$. B: representative image of HUVECs incubated with CM and stained with live/dead staining. 


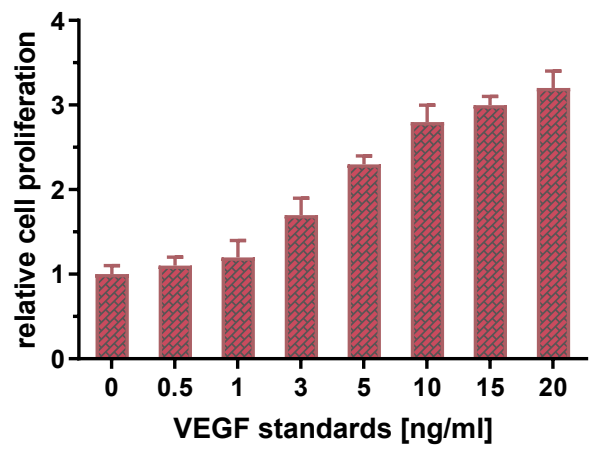

FIGURE S3: Relative cell proliferation of VEGF concentrations ranging from $0.5-20 \mathrm{ng} / \mathrm{ml}$ as measured by HUVEC Alamar Blue proliferation assay.

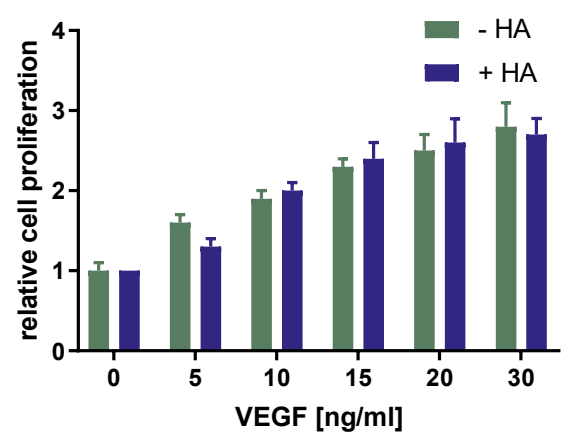

FIGURE S4: Effect of hyaluronic acid ( $\mathrm{HA} ; 8 \mu \mathrm{g} / \mathrm{ml})$ on the relative cell proliferation of HUVEC stimulated by VEGF concentrations of 0 - $30 \mathrm{ng} / \mathrm{ml}$, as measured by Alamar Blue proliferation assay. The ratio (W/W) of VEGF:HA ranged from 1:1600 (5 ng/ml VEGF) to 1:267 (30 ng/ml VEGF). Green bars represent the relative cell proliferation induced by VEGF without added HA ("- HA"), blue bars represent the relative cell proliferation by VEGF with added $8 \mu \mathrm{g} / \mathrm{ml} \mathrm{HA}$ $("+H A)$. 


\section{Chapter 4}

\section{Self-encapsulation of Proangiogenic Growth Factors in PLCA Microspheres}

Karina C. Scheiner', Roel F. Maas-Bakker', Mies ]. van Steenbergen', Steven P. Schwendeman'², Wim E. Hennink', Robbert ]. Kok 


\section{Abstract}

Purpose: To investigate active self-encapsulation (ASE), a post-loading method based on binding of (positively charged) proteins to microporous PLGA microspheres loaded with negatively charged polysaccharides (trapping agents), for simultaneous loading and sustained release of proangiogenic growth factors.

Methods: Vascular endothelial growth factor (VEGF), fibroblast growth factor (FGF) and insulinlike growth factor (IGF) were loaded in microspheres through incubation of porous PLGA microspheres containing dextran sulfate (HDS) as trapping agent in a concentrated growth factor solution of low ionic strength and of $\mathrm{pH} 5$ under conditions the protein is positively charged to allow its binding to the negatively charged trapping agent. Subsequent pore closure was induced by incubation of the growth factor-loaded microspheres at $42.5^{\circ} \mathrm{C}$, i.e. above the $T_{g}$ of (hydrated) PLGA $\left(30^{\circ} \mathrm{C}\right.$ ). The in vitro release kinetics and bioactivity of loaded growth factors were studied for 4 weeks using ELISA and an endothelial cell proliferation assay, respectively.

Results: A combination of VEGF, FGF and IGF was loaded in HDS-containing microspheres with high loading (4.3\%) and loading efficiency (91\%). While IGF was released quickly, VEGF and FGF were continuously released for 4 weeks in their bioactive form, whereby a growth factor combination had a synergistic angiogenic effect.

Conclusion: ASE is a suitable method for co-loading growth factors and providing sustained release of bioactive growth factors for 4 weeks and is therefore an attractive depot formulation for vascularization of biomaterial implants. 


\section{Introduction}

Recombinant growth factors have the potential to regulate and reprogram stem cells and immune cells. ${ }^{1-3}$ Further, recombinant growth factors are promising biotherapeutics within the field of regenerative medicine for functional tissue-engineered constructs. ${ }^{4}, 5$ Tissueengineered constructs are based on biomaterial scaffolds (implants) in which cells are incorporated., 7 Upon transplantation, the formation of blood vessels in the vicinity of the implant is crucial for facilitating the supply of transplanted cells with oxygen and nutrients and to ensure clearance of waste products. ${ }^{8}$ Angiogenesis towards the implants can be stimulated and triggered by loading and release of proangiogenic growth factors into/from the implant, of which vascular endothelial growth factor (VEGF) is the most prominent growth factor. ${ }^{9-11}$ Besides VEGF, also other growth factors play an important role in angiogenesis, such as fibroblast growth factor (FGF) and insulin-like growth factor (IGF). ${ }^{12-14}$ Studies in rodent models have shown that functional vascularization of implants, i.e. the formation of stable blood vessels that encompass the device, takes around four weeks upon implantation. ${ }^{15-17}$ Although continuous growth factor levels in the vicinity of the implant are needed, ${ }^{18}$ their short half-life after systemic administration is a major limitation to achieve persistent tissue levels. ${ }^{19}$ Controlled drug delivery systems, such as polymeric microspheres, offer the possibility of sustained local release of growth factors in a desired time frame. ${ }^{4,11}$ Commonly, proteins, such as growth factors, are formulated in microspheres based on poly(lactic-co-glycolic acid) (PLGA), a well-known and well-characterized biodegradable polymer, by a double emulsion method. ${ }^{20-22}$ However, the applied methods to prepare protein-loaded microspheres (e.g. emulsion evaporation technologies) exposes the protein to shear stresses and organic solvents, which can adversely affect their structural integrity and biological activity. ${ }^{23-25}$

As an alternative, post-loading of the protein into PLGA microspheres can circumvent the limitation described above, foremost by mitigating shear stress and the exposure to organic solvents. An important additional advantage of this method is the possibility to sterilize the microspheres prior to loading the desired compound, thereby avoiding exposure of the compound to sterilization methods, reducing production costs and increasing production efficiency. ${ }^{26}$ The post-loading method active self-encapsulation (ASE) has been developed recently, whereby a negatively charged biopolymer ("trapping agent") loaded in porous PLGA microspheres is used to bind and trap (load) positively charged protein therapeutics through electrostatic interaction under mild conditions (room temperature, buffer of neutral $\mathrm{pH}$ and low ionic strength). Afterwards, the temperature is raised above the glass transition temperature of PLGA which leads to the closure of pores (figure 1). ${ }^{27}, 28$ Upon pore closure, the loaded protein is entrapped (far right schematic microsphere, figure 1) and its release is governed by degradation of the polymeric matrix, rather than by its diffusion through pores of the polymeric matrix (center schematic microsphere in figure 1). During degradation, acidic degradation products are formed, resulting in a decrease of the $\mathrm{pH}$ within the 
polymeric matrix, ${ }^{29,30}$ which in turn might result in loss in bioactivity of the loaded protein. ${ }^{31-33}$ The incorporation of the poorly water-soluble basic additive $\mathrm{MgCO}_{3}$ in the microspheres neutralizes the acid degradation products and thus prevents the acidification of polymeric matrix during degradation. ${ }^{30,33-35}$

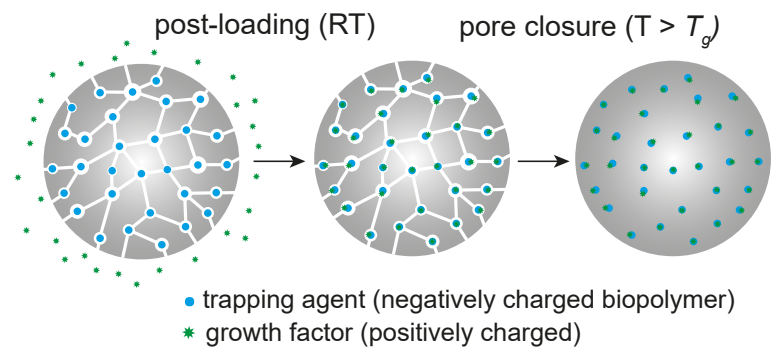

FIGURE 1: Schematic image of the active self-encapsulation post-loading method. ${ }^{27}$ First, negatively charged trapping agent-containing porous PLGA microspheres are incubated at room temperature with a growth factor in a buffer of low ionic strength at which the protein carries a positive charge at room temperature, whereby the positively charged growth factor (green star) is loaded into the pores of the microspheres through electrostatic interaction with the negatively charged biopolymer (trapping agent, in blue). Subsequently, the pores of the microspheres are closed at a temperature above the glass transition temperature of PLGA.

ASE has been achieved with a high loading content (up to $4.5 \mathrm{wt} \%$ ), high loading efficiency (up to 100\%) in PLGA microspheres of the model protein lysozyme (a protein of 14kDa and a pl of $\left.11.4^{36}\right)$. Further, growth factors VEGF and FGF were loaded with high loading and loading efficiency in PLGA microspheres using high molecular weight dextran sulfate (HDS) as trapping agent. ${ }^{28}$ Although these studies have shown the potential to encapsulate growth factors into PLGA microspheres, a combination of several growth factors will have stronger proangiogenic effects and it therefore seems attractive to create slow-releasing particles which are co-loaded with an optimal blend of growth factors that can induce angiogenesis..$^{37-39}$

In this study, we investigated the loading and subsequent release of VEGF in PLGA microspheres using three different trapping agents, i.e. the anionic polysaccharides dextran sulfate, hyaluronic acid and chondroitin sulfate. We also explored the possibility of loading individual growth factors (i.e. VEGF, FGF and IGF) as well as the combination of such growth factors in one microsphere formulation. We studied the subsequent release of the loaded growth factors from the microspheres and assessed their in vitro bioactivity with an endothelial cell proliferation assay. 


\section{Experimental section}

\section{Materials}

PLGA (50:50, lauryl end-capped, i.v. 0.59 dL/g) was purchased from Lactel Durent Corporation (Birmingham, AL, USA). PVA (9,000 - 10,000 kDa, 80\% hydrolyzed), D-(+)-trehalose dihydrate, high molecular weight dextran sulfate $(\sim 500 \mathrm{kDa})$, fluorescein isothiocyanate (FITC)dextran sulfate sodium ( $500 \mathrm{kDa}$ ), sodium phosphate monobasic monohydrate $\left(\mathrm{NaH}_{2} \mathrm{PO}_{4}\right.$. $\left.\mathrm{H}_{2} \mathrm{O}\right)$, bovine serum albumin $(\mathrm{BSA})$, magnesium carbonate $\left(\mathrm{MgCO}_{3}\right)$, sodium azide $\left(\mathrm{NaN}_{3}\right)$ and succinic acid were purchased from Sigma Aldrich (St. Louis, MO, USA). Tween 20 was purchased from Fisher Scientific (Fair Lawn, NJ, USA). Cyanine 5 (Cy5)-labelled lysozyme was purchased from NanoCS (New York, NY, USA). Chondroitin sulfate sodium salt from shark cartilage ( $63 \mathrm{kDa}$ ) and hyaluronic acid sodium salt from Streptococcus equi (50 - $70 \mathrm{kDa}$ ) were purchased from Sigma Aldrich (Zwijndrecht, the Netherlands). Recombinant human vascular endothelial growth factor $\left(\mathrm{VEGF}_{165}, 38.2 \mathrm{kDa}\right)$ was purchased from PeproTech Inc. (Rocky Hill, NJ, USA). Recombinant human insulin-like growth factor (IGF-1, 7.6 kDa) and recombinant human fibroblast growth factor (FGF2, basic FGF, $16 \mathrm{kDa}$ ) were purchased from R\&D Systems (Abington, United Kingdom). Gibco Dulbecco's Phosphate Buffered Saline (DPBS, 10x; when diluted to a 1X working concentration, PBS contains $137 \mathrm{mM} \mathrm{NaCl}, 2.7$ $\mathrm{mM} \mathrm{KCl}, 8 \mathrm{mM} \mathrm{Na}_{2} \mathrm{HPO}_{4}$, and $2 \mathrm{mM} \mathrm{KH}_{2} \mathrm{PO}_{4}$ ) was purchased from Thermo Fisher Scientific (Amsterdam, the Netherlands). Collagen I (from rat tail) was obtained from Corning (Corning, NY, USA). All other reagents and solvents were obtained from Sigma Aldrich, if not specified differently.

\section{Methods}

\section{Preparation of microspheres}

Trapping agent-loaded PLGA microspheres were prepared by a double emulsion solvent evaporation method as described previously. ${ }^{28}$ The trapping agents used in this study were high molecular weight dextran sulfate (HDS) or FITC-labelled high molecular weight dextran sulfate (FITC-HDS), chondroitin sulfate (CS) and hyaluronic acid (HA) (chemical structures are shown in figure 2).

A solution of $250 \mathrm{mg} / \mathrm{ml}$ PLGA in $1 \mathrm{ml} \mathrm{DCM}$ supplemented with $3 \mathrm{wt} \% \mathrm{MgCO}_{3}$ (particle size $\leq$ $90 \mu \mathrm{m}$, wt\% referring to PLGA) was emulsified with $200 \mu$ l of a trapping agent solution in water (4 wt\% target loading, supplemented with 3 wt\% trehalose) using a Virtis Tempest I.Q. ${ }^{2}$ Sentry Microprocessor homogenizer (Triad Scientific, Manasquan, NJ, USA; settings: 18,000 rpm, 60 seconds) in an ice bath. Next, $2 \mathrm{ml}$ of a 5\% PVA solution was added to the primary emulsion and vortexed for 60 seconds. The resulting double emulsion was added to $100 \mathrm{ml}$ of $0.5 \%$ PVA solution in water and stirred for 3 hours for DCM evaporation. The hardened microspheres 
were sieved (63 $\mu$ m-mesh and $20 \mu$ m-mesh sieves, Newark Wire Cloth Company, Newark, NJ, USA) to collect the microspheres fraction of 20 to $63 \mu \mathrm{m}$. Next, the microspheres were washed with double-distilled water and freeze-dried overnight. For microspheres prepared without trapping agents ("none"), a $1 \mathrm{ml}$ solution of $250 \mathrm{mg} / \mathrm{ml}$ PLGA in DCM supplemented with 3 wt $\% \mathrm{MgCO}_{3}$ was emulsified with $200 \mu$ of water, followed by the preparation steps described above. The size distribution of the microspheres was determined by optical particle sizing (Accusizer 780, Santa Barbara, California, USA).

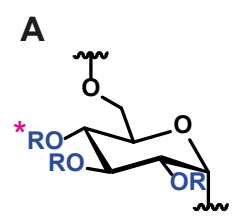

$\mathbf{R}=\mathbf{H}$ or $\mathrm{So}_{3}^{\ominus}$
B

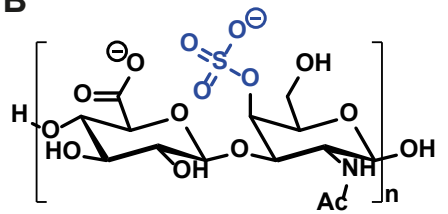

C

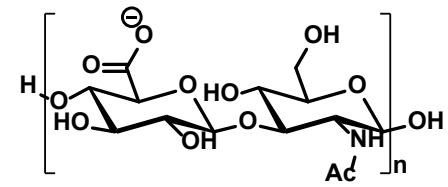

FIGURE 2: Chemical structures of trapping agents loaded in the PLGA microspheres. A:) dextran sulfate, ${ }^{*}$ site of FITC-labelling, degree of substitution $0.001-0.006$ mol FITC / mol glucose (as stated by the supplier), B: chondroitin sulfate, C: hyaluronic acid.

\section{Elemental analysis of trapping agents}

The sulfur content of trapping agents dextran sulfate and chondroitin sulfate was determined by elemental analysis. Accurately weighed samples of $5 \mathrm{mg}$ were analyzed on a Vario Micro Cube elemental analyzer in CHNS mode (Elementar GmbH, Langenselbold, Germany) according to supplier's recommended equipment settings.

\section{Active self-encapsulation of growth factors in microspheres}

Growth factor solutions $(1.0 \mathrm{mg} / \mathrm{ml}$ of VEGF, FGF, IGF or a $0.33 / 0.33 / 0.33 \mathrm{mg} / \mathrm{ml}$ combination of VEGF, FGF and IGF) were prepared by reconstituting lyophilized growth factors (as received by the supplier, molecular weights and isoelectric points stated in table 1) in ASE loading buffer, consisting of $5 \mathrm{mM}$ succinate, $275 \mathrm{mM}$ trehalose (pH 5). Next, $20 \mathrm{mg}$ of freeze-dried PLGA microspheres with and without loaded trapping agent were suspended in $1 \mathrm{ml}$ of growth factor solution and incubated for 48 hours at room temperature $\left(\sim 21^{\circ} \mathrm{C}\right)$ under mild agitation. Then, the sample was incubated for 42 hours at $42.5^{\circ} \mathrm{C}$ under mild agitation to induce pore closure. Afterwards, the sample was centrifuged at 8000 rpm (5724 x g, Sigma 1-16K, Sigma Laborzentrifugen GmbH, Osterode am Harz, Germany) for 5 minutes and the supernatant was collected for determination of the fraction non-loaded growth factor (see section Determination of growth factor loading by Coomassie Plus assay). The microspheres were washed twice with $1 \mathrm{ml}$ of deionized water and freeze-dried overnight. 
TABLE 1: Molecular weights and isoelectric points of proteins used in this study.

\begin{tabular}{ccc}
\hline protein & $\begin{array}{c}\text { molecular weight } \\
{[\mathbf{k D a}]^{\mathbf{a}}}\end{array}$ & $\begin{array}{c}\text { isoelectric point } \\
(\boldsymbol{p} \mathbf{l})\end{array}$ \\
\hline lysozyme (LYZ) & 14 & $11.4^{36}$ \\
\hline vascular endothelial growth factor (VEGF) & 38.2 & $8.5^{40}$ \\
fibroblast growth factor (FGF) & 16 & $9.6^{41}$ \\
insulin-like growth factor 1 (IGF) & 7.6 & $8.5^{42}$ \\
\hline
\end{tabular}

a: as stated by supplier.

\section{Determination of growth factor loading by Coomassie Plus assay}

The growth factor content of the microspheres was determined indirectly by quantifying the protein content in supernatants collected after ASE by Coomassie Plus protein assay (Thermo Scientific, Amsterdam, the Netherlands). In short, $10 \mu$ of growth factor standards or supernatant samples were pipetted into the wells of a 96-well plate. Next, $200 \mu$ of Coomassie Plus reagent was added to the wells. The plate was incubated for 10 minutes at room temperature after which the absorbances of the wells at $595 \mathrm{~nm}$ was recorded using a SPECTROstar Nano plate reader (BMG Labtech, Ortenberg, Germany).

\section{Scanning electron microscopy}

The morphology of freeze-dried microspheres was analyzed by scanning electron microscopy (SEM; Phenom, FEl Company, Eindhoven, the Netherlands). Lyophilized microspheres were transferred onto 12-mm diameter aluminum specimen stubs (Agar Scientific Ltd., Essex, United Kingdom) using double-sided adhesive tape. Next, the samples were frozen in liquid nitrogen and cut with a razor. Prior to analysis, the microspheres were coated with platinum using an ion coater under vacuum.

\section{Confocal fluorescence microscopy}

FITC-HDS loaded PLGA microspheres were prepared as described in section Preparation of microspheres. The microspheres were loaded with cyanine-5-labelled lysozyme (Cy5-LYZ) at $1 \mathrm{mg} / \mathrm{ml}$ concentration in $10 \mathrm{mM}$ phosphate $\left(\mathrm{NaH}_{2} \mathrm{PO}_{4} \cdot \mathrm{H}_{2} \mathrm{O}\right)$ buffer ( $\mathrm{pH}$ 7.0) according to the active self-encapsulation method as described in section Active-selfencapsulation of growth factors in microspheres. Freeze-dried Cy5-LYZ-loaded microspheres were fixed with ProLong Gold antifade reagent (Invitrogen, Thermo Fisher Scientific, Eugene, USA) on a glass slide for 30 minutes. FITC-HDS and Cy5-LYZ in microspheres were imaged using a Leica SP8 confocal fluorescence microscope (Leica, Buffalo Grove, USA; FITC-HDS (green channel) at $\lambda_{\text {ex }} 492 \mathrm{~nm}$ and $\lambda_{\text {em }} 518 \mathrm{~nm}$; Cy5 (red channel) at $\lambda_{\text {ex }} 650 \mathrm{~nm}$ and $\lambda_{\text {em }} 670 \mathrm{~nm}$ ). 


\section{Formulations for investigating the effect of ionic strength and pore closure on VECF loading} Experiments were performed to study the effect of ionic strength of the buffer on the loading of VEGF in microspheres containing HDS as trapping agent, without subsequent pore closure. HDS-containing microspheres (prepared as described in section Preparation of microspheres) were incubated with $1 \mathrm{mg} / \mathrm{ml}$ VEGF in either ASE buffer (5 mM succinate, 275 mM trehalose (pH 5)), 10 mM HEPES pH 7.4 or Dulbecco's PBS pH 7.4 (composition stated in Materials section) for 48 hours at room temperature under mild agitation. Afterwards, the suspension was centrifuged at 8000 rpm for 5 minutes (5724 x g, Sigma 1-16K, Sigma Laborzentrifugen GmbH, Osterode am Harz, Germany) the supernatant was collected, and the microspheres were washed twice with $1 \mathrm{ml}$ of deionized water. The protein concentration in the supernatants was determined by the Coomassie Plus assay (see section Determination of growth factor loading by Coomassie Plus assay).

To study adsorption of VEGF on the surface of microspheres, the pores of HDS-containing microspheres were closed in a two-step process, similar to the ASE process described in section Determination of growth factor loading by Coomassie Plus assay. HDS-containing microspheres were incubated in $1 \mathrm{ml}$ of ASE buffer $(5 \mathrm{mM}$ succinate, $275 \mathrm{mM}$ trehalose ( $\mathrm{pH}$ 5)) for 48 hours at room temperature, followed by incubation at $42.5^{\circ} \mathrm{C}$ for 42 hours. Then, the suspension was centrifuged at 8000 rpm for 5 minutes and the microspheres were subsequently washed twice with $1 \mathrm{ml}$ of deionized water and freeze-dried overnight. Next, the freeze-dried microspheres were incubated with $1 \mathrm{ml}$ of a $1 \mathrm{mg} / \mathrm{ml}$ VEGF solution in ASE buffer (5 mM succinate, 275 mM trehalose ( $\mathrm{pH}$ 5.0)) for 48 hours at room temperature under mild agitation. The suspension was then centrifuged and washed as described above, after which the unbound VEGF content was determined in supernatant as described in section Determination of growth factor loading by Coomassie Plus assay.

\section{In vitro release characteristics of VEGF-loaded microspheres}

Five mg microspheres were accurately weighed and suspended in $1 \mathrm{ml}$ of in vitro release buffer ("IVR buffer"), which consisted of Dulbecco's PBS pH 7.4, supplemented with $10 \mathrm{mg} /$ $\mathrm{ml} \mathrm{BSA}, 0.02 \%$ Tween 80 and $0.02 \% \mathrm{NaN}_{3}$. The samples were incubated at $37{ }^{\circ} \mathrm{C}$ under gentle agitation. At different time points, the samples were centrifuged at 8000 rpm for 5 minutes (5724 x g, Sigma 1-16K, Sigma Laborzentrifugen GmbH, Osterode am Harz, Germany). The supernatant was removed and replaced by fresh release buffer. The concentration of growth factor in the release samples was quantified with ELISA (section Quantification of released growth factors by ELISA). 


\section{Bioactivity of released growth factors}

Samples of released growth factors were obtained by incubating $1 \mathrm{mg}$ of accurately weighted microspheres in $1 \mathrm{ml}$ of "bioactivity IVR buffer" (consisting of Dulbecco's PBS pH 7.4 , supplemented with $0.5 \%$ BSA, $30 \mu \mathrm{g} / \mathrm{ml}$ gentamicin and $15 \mathrm{ng} / \mathrm{ml}$ amphotericin, 0.2 $\mu \mathrm{m}$ filtered) at $37^{\circ} \mathrm{C}$ under gentle agitation. At appropriate time points, the samples were centrifuged at 8000 rpm for 5 minutes (5724 x g, Sigma 1-16K, Sigma Laborzentrifugen GmbH, Osterode am Harz, Germany). The supernatant was removed and the release medium replaced by fresh buffer. The collected supernatants were analyzed for growth factor concentration using an ELISA (as described in section Quantification of released growth factors by ELISA) and used for bioactivity assays (as described in sections Bioactivity of released growth factors by endothelial cell proliferation assay and Bioactivity of released VEGF by bioluminescent receptor-based cell assay).

\section{Quantification of released growth factors by ELISA}

IVR samples were analyzed by a sandwich ELISA (Human VEGF DuoSet ELISA, Human FGF basic/FGF2 DuoSet ELISA, Human IGF-I/IGF-1 DuoSet ELISA, all R\&D Systems, Abingdon, United Kingdom) according to the manufacturer's protocol. The stock solutions of the ELISA kits were used for calibration in the concentration range of $31-2000 \mathrm{pg} / \mathrm{ml}$ for VEGF and IGF, or $15.6-1000 \mathrm{pg} / \mathrm{ml}$ for FGF. Release samples were diluted with reagent diluent to fall within the working range of the assay and measured in duplicate. ELISA plates were read at $450 \mathrm{~nm}$ using a SPECTROstar Nano plate reader (BMG Labtech, Ortenberg, Germany).

\section{Bioactivity of released growth factors by endothelial cell proliferation assay}

The bioactivity of released growth factors from microspheres was analyzed by Alamar Blue proliferation assay with human umbilical vein endothelial cells (HUVECS), as described previously. ${ }^{43}$ The assay was performed in growth factor-poor medium ("bioactivity medium"), consisting of M199 medium supplemented with 2.5\% fetal bovine serum (FBS). In brief, HUVECs were seeded at 4000 cells/well in collagen-coated 96 well plates. Release samples and standards, diluted in bioactivity medium, were added to the wells and the plate was incubated for 96 hours at $37{ }^{\circ} \mathrm{C} / 5 \% \mathrm{CO}_{2}$. The fluorescence, caused by the proliferating cells after addition of Alamar Blue, was measured with a microplate reader $\left(\lambda_{\mathrm{ex}} 530 \mathrm{~nm}\right.$ and $\lambda_{\text {em }} 600 \mathrm{~nm}$; Berthold Mithras LB 940, Germany).

Release samples were diluted in bioactivity medium to fall within the responsive range of unformulated VEGF, i.e. corresponding to a concentration of 1-10 $\mathrm{ng} / \mathrm{ml}$ VEGF based on loading data. A calibration curve with non-formulated VEGF was fitted with by a four parameter logistic regression model using GraphPad prism 7, and used to calculate the concentrations of VEGF in the release samples which were subsequently used to determine the cumulative release of bioactive VEGF normalized to the total release of bioactive VEGF at the end of the IVR study (time point 28 days). 
For testing the bioactivity of growth factors VEGF, FGF and IGF released from microspheres, release samples were diluted in bioactivity medium to fall within the responsive range of VEGF, FGF and IGF combined in a 1:1:1 weight ratio, corresponding to a relative cell proliferation of 1-9.

\section{Bioactivity of released VEGF by bioluminescent receptor-based cell assay}

The bioactivity of released VEGF was additionally analyzed by a bioluminescent receptorbased cell assay (VEGF Bioassay, Promega, Madison, WI, USA) according to the manufacturer's instructions. This assay relies on the binding of VEGF to the VEGFR-2 receptor (also known as kinase insert domain receptor (KDR)) on KDR/NFAT-RE HEK 293 cells, which induces a luminescent response via intracellular NFAT-RE signaling. ${ }^{44}$ In brief, KDR/NFAT-RE HEK 293 cells were thawed, reconstituted and seeded in 96-well plates in assay medium (DMEM with 10\% FBS, according to the manufacturer's protocol). Non-formulated VEGF was added in concentrations 0 - 1000 ng/ml, as well as IVR supernatants diluted 1200 - 6000-fold in assay buffer. Reporter cells were incubated with calibration or IVR samples for 6 hours at $37^{\circ} \mathrm{C}$ before adding Bio-GloTM reagent. After incubation at room temperature for 5-10 minutes, luminescence was measured on a microplate reader (Mithras LB 940, Berthold, Bad Wildbad, Germany) using an integration time of 0.25 seconds/well. Calibration and IVR samples were measured in triplicate. Results are shown as relative proliferative signals defined by the luminescence signal normalized by the luminescence signal of cells incubated without VEGF. A calibration line with non-formulated VEGF was fitted by a 4PL curve-fit with GraphPad prism 7, and used to determine the cumulative release of bioreceptor-assay active VEGF normalized to the total release of bioreceptor-assay active VEGF at the end of the IVR study (time point 28 days).

\section{Statistical analysis}

Data are presented as average with SD. Statistical analysis was performed with GraphPad Prism7 using the Holm-Sidak multicomparison test. Differences between the analyzed groups were considered significant if $\mathrm{p}<0.05$. 


\section{Results and Discussion}

\section{Loading of VEGF in trapping agent-containing microspheres}

The aim of this study was to load proangiogenic growth factors by active self-encapsulation (ASE) in PLGA microspheres using the negatively charged biopolymers dextran sulfate (HDS), chondroitin sulfate (CS) and hyaluronic acid (HA) as trapping agents. These polysaccharides were selected for their binding capacities with positively charged proteins, such as the proteins used in this study, VEGF, FGF, IGF and lysozyme (LYZ) ( $p /$ reported in table I). Porosity of PLGA microspheres was introduced through the addition of sugar-leaching agent trehalose, ${ }^{27}$ which also served as lyoprotectant. ${ }^{45}$ Magnesium carbonate $\left(\mathrm{MgCO}_{3}\right)$ was added as acidneutralizing agent. Of the three trapping agents used in this study, dextran sulfate had the highest molecular weight (500 kDa) and highest number of sulfate groups (1.75 per dextran unit, sulfur content (14\%)), as shown in table 2 . Chondroitin sulfate and hyaluronic acid had lower average molecular weights, i.e. $63 \mathrm{kDa}$ and $60 \mathrm{kDa}$, and lower or none sulfate content, i.e. 5 \% (for CS; corresponding to 0.7 sulfate group per disaccharide unit) and $0 \%$ (for HA).

Lyophilized trapping agent-containing microspheres had comparable size and polydispersity, as reported in table II. In the first step of the active self-encapsulation method, microspheres were incubated in a concentrated growth factor solution of $\mathrm{pH}$, at which the loaded proteins have a positive charge. The growth factor solution had a low ionic strength and also contained trehalose as lyoprotectant. Subsequently, the pores of the PLGA microparticle matrix were closed by incubating the microsphere dispersions at temperature of $42.5^{\circ} \mathrm{C}$ which is above the glass transition temperature $\left(T_{g}\right)$ of the polymer (i.e. $\sim 30^{\circ} \mathrm{C}$ hydrated polymer, $\sim 45^{\circ} \mathrm{C}$ dry polymer ${ }^{46,47}$ ).

The highest VEGF loading and loading efficiency, i.e. 4.5 wt \% loading and nearly 100\% loading efficiency, was measured for HDS-containing microspheres. A lower VEGF loading and loading efficiency, i.e. 1.3 wt \% loading and $28 \%$ loading efficiency, was measured for CS-containing microspheres. No VEGF was loaded in HA-containing microspheres as well as placebo microspheres without trapping agent.

Microsphere morphologies before and after ASE, in the legend described as before and after pore closure, are shown as SEM images in figure 3. Before ASE all microsphere formulations showed a porous morphology, both on the surface and inside of the microspheres (figure 3 , left column). After the pore closure step, the surfaces of all microsphere formulations were smooth (figure 3, right column). Cross-sections of microspheres after pore closure revealed a less porous network, i.e. showing fewer small pores in the core of the particles, as compared to cross-sections of microspheres before pore closure. 
TABLE 2: Characteristics of biopolymers high molecular weight dextran sulfate (HDS), chondroitin sulfate (CS) and hyaluronic acid (HA) used as trapping agents for microspheres (average molecular weight and sulfur content) and VEGF loading and loading efficiency in microspheres containing either trapping agents HDS, CS, HA or no trapping agent. (average $\pm S D$ of $n=3$ loading experiments)

\begin{tabular}{cccccc}
\hline Trapping agent & $\begin{array}{c}\text { molecular } \\
\text { weight [kDa] }\end{array}$ & $\begin{array}{c}\text { Sulfur content } \\
\text { [\%] }^{\mathbf{a}}\end{array}$ & $\begin{array}{c}\text { MSPs size } \\
\text { [average } \pm \text { SD] }\end{array}$ & $\begin{array}{c}\text { VEGF loading } \\
\text { content } \\
\text { [wt \%] }^{\mathbf{c}}\end{array}$ & $\begin{array}{c}\text { VEGF loading } \\
\text { efficiency [\%] }\end{array}$ \\
\hline HDS & 500 & 14 & $72 \pm 23 \mu \mathrm{m}$ & $4.5 \pm 0.0$ & $98 \pm 0$ \\
CS & $\sim 63$ & 5 & $70 \pm 22 \mu \mathrm{m}$ & $1.3 \pm 0.6$ & $28 \pm 13$ \\
HA & $50-70$ & 0 & $81 \pm 23 \mu \mathrm{m}$ & 0 & 0 \\
none & n.a. ${ }^{*}$ & n.a.* $^{*}$ & $67 \pm 32 \mu \mathrm{m}$ & 0 & 0 \\
\hline
\end{tabular}

a: determined by elemental analysis, ${ }^{b}$ volume-weight size distribution, $c$ : indirect determination by protein quantification of the supernatant by Coomassie Plus assay, ${ }^{*}$ not applicable. MSPs: microspheres.

Several aspects play a role in the differences in VEGF loading efficiency found for the different trapping agents. An important aspect is the density of sulfate groups of the trapping agents, as VEGF binding increases with increasing degrees of sulfation. ${ }^{48}$ In our study, HDS contained the highest number of sulfate groups per monomer unit, compared to CS. Moreover, HA does not contain sulfate groups but has carboxylate groups which apparently did not facilitate VEGF trapping. Our results hence show a clear correlation between sulfate density of the trapping agent and ASE loading of VEGF in line with a previous study. ${ }^{48}$

Another possible explanation for the lower VEGF loading efficiencies in CS- and HAmicrospheres could be due to differences in loading efficiencies of the trapping agents themselves, which may correlate to their respective molecular weights.

Unfortunately, our attempts to quantify the loading efficiencies of the trapping agent were not successful. Among others, we have tested the dissolution and destruction of microspheres in a high pH buffer or organic solvent, followed by GPC analysis. However, no peaks for trapping agents were observed, not even for HDS- and CS-loaded microspheres which displayed good ASE properties and hence provided indirect evidence of the successful entrapment of trapping agents. 
before pore closure
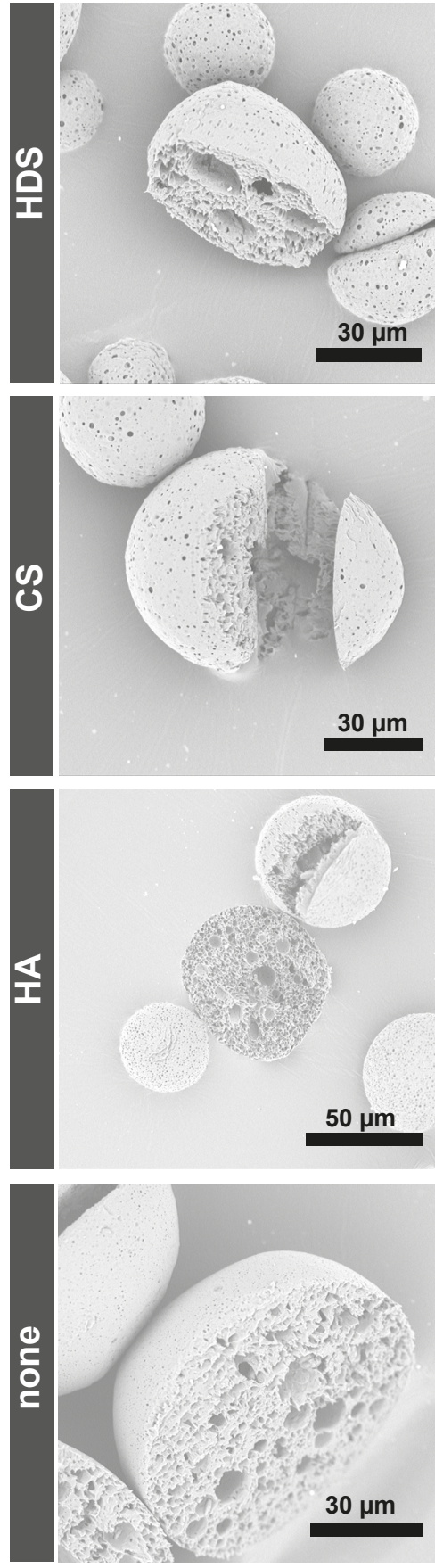

\section{after pore closure}
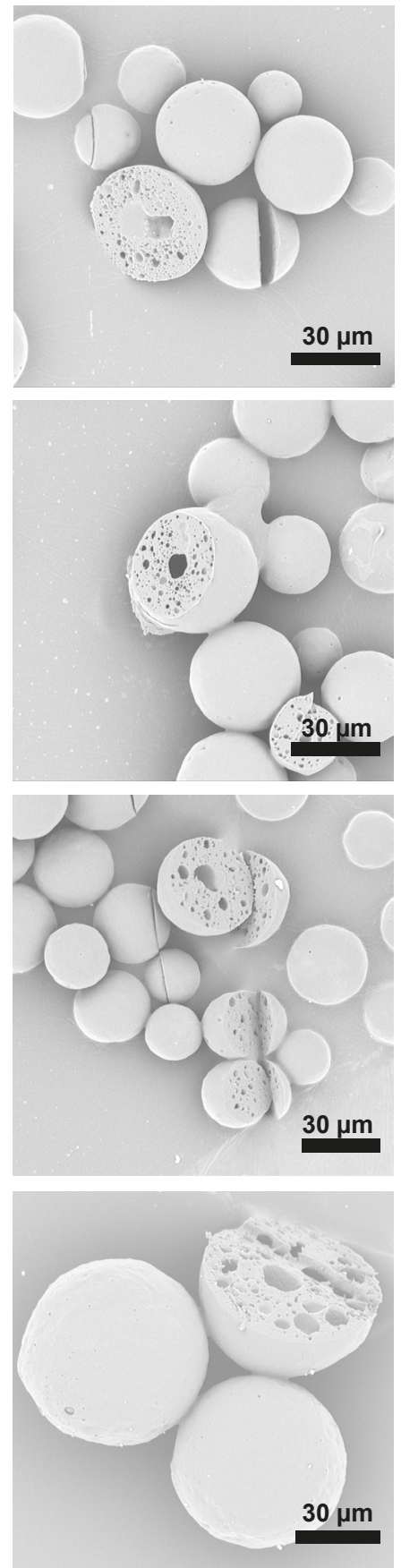

FIGURE 3: SEM images of PLGA microspheres containing trapping agents HDS, CS, HA or no trapping agent ("none") before and after pore closure (loading of VEGF). The trapping agent per microsphere formulation is stated on the left column (HDS: high molecular weight dextran sulfate; CS: chondroitin sulfate; HA: hyaluronic acid; none: no trapping agent used). 
In order to demonstrate the distribution of trapping agent and ASE-loaded proteins within the microspheres, we prepared microspheres with fluorescently labelled HDS. The distributions of FITC-HDS and Cy5-labelled LYZ are shown in figure 4, demonstrating that both components (protein and trapping agent) are evenly distributed in the microsphere matrix. Microspheres used for this experiment were comparable to the microspheres used for VEGF loading.

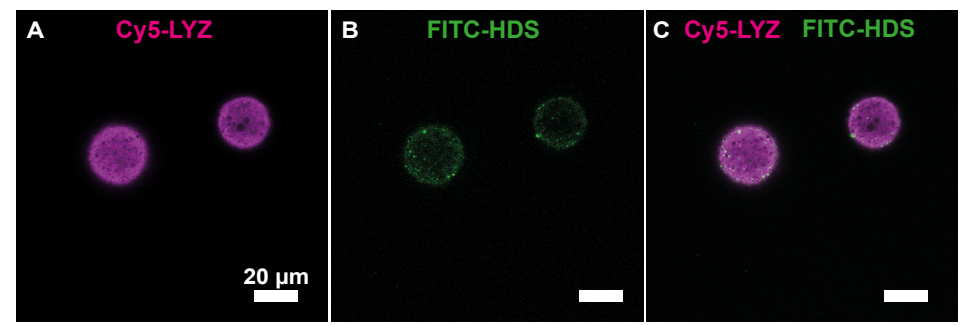

FIGURE 4: Confocal fluorescence microscopy of Cy5-LYZ-loaded PLGA microspheres prepared with the trapping agent FITC-HDS. A: red channel shows Cy5-LYZ in microspheres, B: green channel shows FITC-HDS in microspheres, C: merge of red and green channels showing Cy5-LYZ and FITC-HDS. White scale bar represents $20 \mu \mathrm{m}$.

\section{Effect of ionic strength and pore closure on VEGF loading in microspheres}

The effect of the ionic strength of the loading buffer used on the VEGF loading efficiency [\%] (without pore closure) is shown in table 3. The highest VEGF loading efficiency (92\%) was measured with ASE loading buffer (5 mM succinate, $275 \mathrm{mM}$ trehalose, pH 5). A slightly lower VEGF loading efficiency (60\%) was measured with a loading solution at higher $\mathrm{pH}(\mathrm{pH} 7.4)$ at similar ionic strength (10 mM HEPES). Due to VEGF's isoelectric point (8.5), VEGF exhibits a higher positive charge at $\mathrm{pH} 5$ than at $\mathrm{pH} 7.4$ and therefore interacts stronger with $\mathrm{HDS}$ than at $\mathrm{pH}$, leading to a higher loading efficiency. No VEGF was loaded when PBS pH 7.4 (with 150 mM ionic strength) was used as loading buffer. Most likely, the higher salt concentration shields the charges of both the trapping agent and the protein, thereby preventing ionic interaction ${ }^{49}$

Further, HDS-containing microspheres after pore closure showed only low VEGF loading efficiency of $2 \%$ after incubation of VEGF in ASE buffer ( $5 \mathrm{mM}$ succinate, $275 \mathrm{mM}$ trehalose, $\mathrm{pH}$ 5) (table 3). This result confirmed that incubation of microspheres above the $T_{g}$ of the polymer closes the pores and as a consequence changes the accessibility of the trapping agent, leaving only a small amount of surface-accessible binding sites for VEGF. Collectively, these data show that ASE is indeed a ionic strength-driven process, where interaction of VEGF with available HDS/trapping agents in the porous polymer matrix is essential for VEGF loading. 
TABLE 3: Loading of VEGF in HDS-containing microspheres with varied ionic strength buffer and after previous microsphere pore closure. (average \pm SD $(n=2)$ )

\begin{tabular}{ccc}
\hline loading conditions & loading buffer & $\begin{array}{c}\text { VEGF loading efficiency } \\
\text { [\%] }\end{array}$ \\
\hline $\begin{array}{c}\text { incubation 48 } \mathrm{h} \text { at RT, no } \\
\text { pore closure }\end{array}$ & $5 \mathrm{mM}$ succinate, $275 \mathrm{mM}$ trehalose, pH 5 (ASE buffer) & $92 \pm 6$ \\
\hline $\begin{array}{c}\text { pore closure, then } \\
\text { incubation 48 h at RT }\end{array}$ & $5 \mathrm{mM}$ succinate, 275 mM trehalose, pH 5 (ASE buffer) & $0 \pm 4$ \\
\hline
\end{tabular}

RT: room temperature

\section{VEGF release from microspheres containing CS or HDS as trapping agent}

The in vitro release of VEGF from CS- or HDS-containing PLGA microspheres was studied for 28 days which time frame has been shown to be advantageous for growth factor-induced vascularization of tissue-engineered constructs. ${ }^{15-17}$ The cumulative release, as measured by ELISA, is shown in figure 5. As previous studies with the VEGF ELISA demonstrated issues with quantitative recovery of the loaded VEGF, ${ }^{43}$ we normalized the release curves to the final cumulative release observed at the end of the incubation period (i.e. day 28). Based on ELISA detection, 10 and $40 \%$ of the loaded VEGF was recovered at day 28 for HDS- and CS-containing microspheres, respectively. This low recovery might be ascribed to an underestimation of the protein concentrations in the release sample as determined by ELISA ${ }^{43}$ and that part of the proteins are is still entrapped in the microspheres at day 28; the remaining VEGF will be released when the PLGA microspheres are fully degraded (2-3 months). ${ }^{50-52}$

VEGF was released continuously from both CS- and HDS-containing microspheres in the time frame of 28 days (figure 5, release curves were fitted with the Korsmeyer-Peppas model ${ }^{53}$, as an indicator for diffusion-controlled release). The high initial release during the first day of incubation is likely caused by superficially encapsulated VEGF. ${ }^{4}$

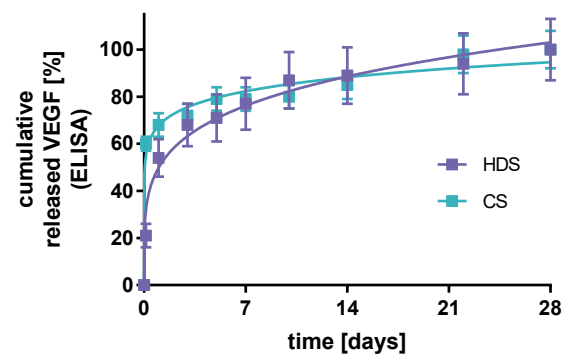

FIGURE 5: Cumulative release of VEGF from PLGA microspheres prepared with trapping agents HDS or CS. In vitro release studies were performed at $37^{\circ} \mathrm{C}$ in Dulbecco's PBS pH 7.4, supplemented with $10 \mathrm{mg} / \mathrm{ml}$ BSA, 0.02\% Tween 80 and $0.02 \% \mathrm{NaN}_{3}$ under gentle agitation. The VEGF concentration in the release samples was quantified by ELISA. The cumulative release of VEGF was normalized to the cumulative amount of protein released at day 28. 


\section{Bioactivity of released VEGF}

The bioactivity of released VEGF is shown in figure 6 . We used a proliferation assay in which endothelial cells were incubated in growth-factor deprived medium spiked with aliquots of the release samples. After dilution of the supernatants towards the proliferation range of 0-10 ng/ $\mathrm{ml}$ of non-formulated VEGF, all release samples showed 2 to 4 -fold induced proliferation (figure 6a), as compared to HUVEC cultured in control medium without added VEGF. Since each of the diluted release samples gave proliferative responses in the expected concentration range after dilution, we concluded that bioactivity of released VEGF was retained. We reconstructed cumulative release curves of bioactive VEGF (figure 6b), assuming that the specific bioactivity of VEGF (i.e. 2-4-fold proliferative response for 3-5 ng/ml VEGF) of formulated and reference VEGF was similar. Both CS- and HDS-microspheres showed continuous release of bioactive VEGF during the complete 28-day time frame of the experiment, with relative lower initial release ( $\sim 30 \%$ ) as compared to the ELISA detection of figure 5 . The release profiles showed no clear differences between HDS- and CS-microspheres.
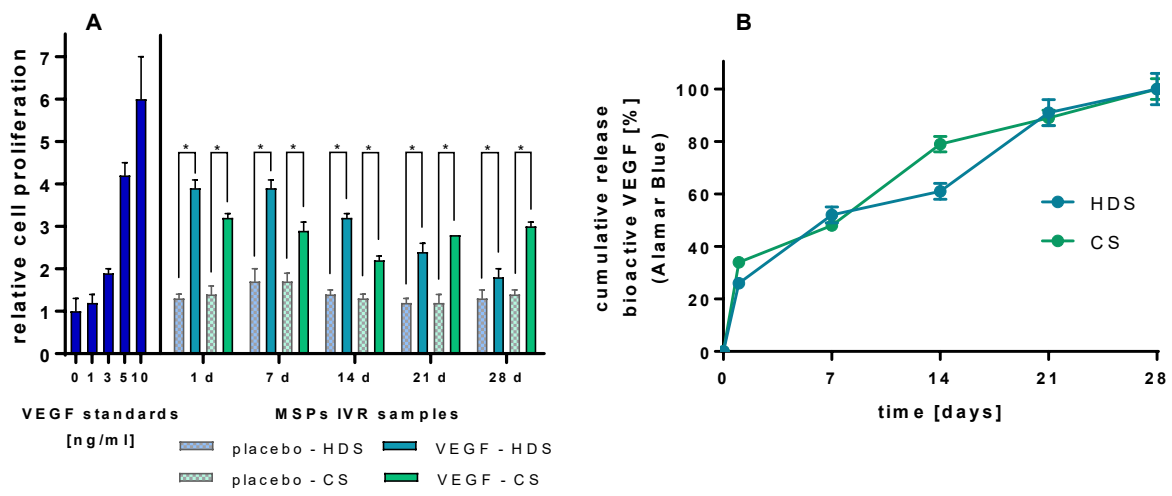

FIGURE 6: Bioactivity of released VEGF released from microspheres prepared with trapping agents HDS or CS. A: relative cell proliferation induced by VEGF standards 0 - 10 ng/ml (blue bars) and release samples ("MSPs IVR samples") from placebo or VEGF-loaded microspheres prepared with trapping agents HDS or CS. Bars represent average \pm SD $(n=3) .{ }^{*} p<0.05$. B: Cumulative release of bioactive VEGF (determined by HUVEC Alamar Blue proliferation assay), normalized to the total amount of bioactive VEGF after 28 days. HDS: dextran sulfate, CS: chondroitin sulfate.

The bioactivity of VEGF was further analyzed by a bioluminescence VEGF reporter assay using HEK 293 cells transfected with the KDR/VEGFR2 receptor. Figure 7 shows the cumulative release curves of bioreceptor-active VEGF, which were reconstructed using the bioluminescence dose-response curve of unformulated VEGF (figure S1). The observed release profiles measured by the bioreceptor assay correspond to the release profile measured by ELISA, with relative high initial release followed by a sustained release phase up to 28 days.

The VEGF recoveries (total protein released at day 28) based on the bioassay were approximately double of those found with ELISA, i.e. $25 \%$ and $70 \%$ versus the loading content of VEGF of HDS 
and CS microspheres, respectively. This result may indicate an underestimation of the amount of released VEGF by ELISA, which can be ascribed to the loss of immunodetectable epitopes in VEGF. While such VEGF molecules may not be detected by ELISA, the bioactivity of released VEGF was, within the experimental error, preserved. Another explanation may be more technical, and relates to the sequential dilution steps that are made to the IVR supernatant towards the working range of the ELISA and the bioluminescence assay.

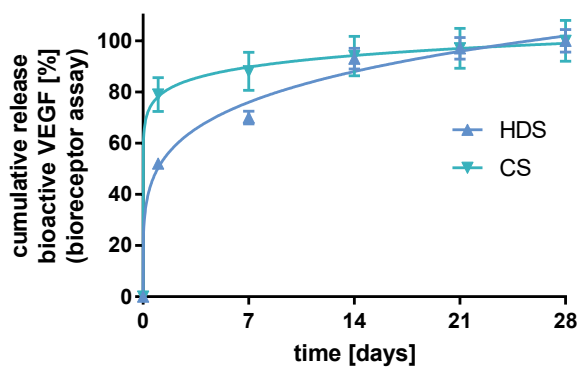

FIGURE 7: Cumulative release of bioactive VEGF from HDS- or CS-microspheres, as measured by the bioluminescence VEGF bioreceptor assay described in Experimental section Bioactivity of released VEGF by bioluminescent receptorbased cell assay. The release was normalized by the total amount of released VEGF after 28 days.

\section{Loading of a combination of proangiogenic growth factors in microspheres}

Based on the more sustained release profile and of high loading efficiency of VEGF by HDScontaining microspheres, we further explored the simultaneous loading of VEGF combined with fibroblast growth factor (FGF) and insulin-like growth factor (IGF-1), all potent angiogenic growth factors ${ }^{12,13}$ with high isoelectric points (table 1). As angiogenesis in vivo is an interplay between many growth factors, delivering a combination of growth factors for therapeutic angiogenesis applications, such as vascularization of a biomaterial implant, is advantageous., 11

Table 4 shows the loading contents and loading efficiencies of FGF and IGF and the simultaneous encapsulation of VEGF, FGF and IGF by ASE loading in HDS microspheres. A total growth factor loading of 4.3 wt\%, corresponding to a loading efficiency of $91 \%$, was achieved for the co-loaded microsphere formulation. SE-UPLC measurements of the post-loading supernatants of the growth factor combination showed a remaining peak at the retention times of IGF, indicating that the non-loaded growth factor fraction consisted primarily of IGF (figure S2). FGF and IGF were also loaded separately into HDS-containing microspheres. FGF loaded microspheres had a loading of 4.6 wt \% and $97 \%$ loading efficiency, while IGF loading in HDS-containing microspheres resulted in a 3.6 wt\% loading and $75 \%$ loading efficiency. Although IGF has approximately the same isoelectric point as VEGF, its ASE entrapment in the microspheres is less efficient. Plausibly, this may be ascribed to its small size (7.6 kDa) and thus lower net number of positive charges per protein molecule as compared to VEGF (38.2 kDa). 
TABLE 4: Loading and loading efficiencies of a combination of VEGF, FGF and IGF, as well as of FGF and IGF separately in microspheres prepared with trapping agent HDS.

\begin{tabular}{ccc}
\hline loaded growth factor(s) & loading content [wt\%] $^{\mathbf{b}}$ & loading efficiency [\%] $^{\text {[ }}$ \\
\hline VEGF + FGF + IGF $\left(1: 1: 1^{\text {a }}\right)$ & $4.3 \pm 0.1$ & $91 \pm 3$ \\
FGF & $4.6 \pm 0.0$ & $97 \pm 0$ \\
IGF & $3.6 \pm 0.2$ & $75 \pm 3$ \\
\hline
\end{tabular}

a: weight ratios, b: indirect determination by Coomassie Plus assay.

The release of simultaneously-loaded growth factors from microspheres was measured by growth factor-specific ELISAs for VEGF, FGF and IGF, respectively (figure 8). VEGF was gradually released over the complete 28-day incubation time frame. In contrast, 10\% of FGF was released during the first week, followed by a sustained release during each consecutive week. IGF was nearly fully released within the first week ( 90\%). Likely, this rapid release is due to weak binding of IGF to the trapping agent and relative entrapment in superficial pores of the microspheres. In line with this assumption, VEGF and FGF were released more sustainably, indicating a deeper diffusion into the porous polymeric network during loading.

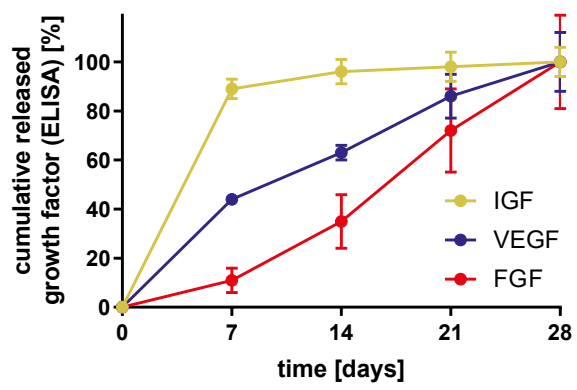

FIGURE 8: Cumulative release [\%] of IGF, VEGF and FGF from microspheres co-loaded with these growth factors. The release was performed at $37^{\circ} \mathrm{C}$ in bioactivity IVR buffer consisting of Dulbecco's PBS pH 7.4, $0.2 \mu \mathrm{m}$ filtered, $0.5 \%$ BSA, $30 \mathrm{\mu g} / \mathrm{ml}$ gentamicin and $15 \mathrm{ng} / \mathrm{ml}$ amphotericin. Released VEGF, FGF or IGF was quantified by specific ELISAs for each protein and normalized to the total cumulative release of each growth factor at day 28 .

\section{Bioactivity of released growth factors}

The bioactivity of the combination of proangiogenic growth factors was studied using the above described endothelial cell Alamar Blue proliferation assay (Materials and Methods section Bioactivity of released growth factors by endothelial cell proliferation assay), as this assay detects a general bioactivity in endothelial cells, while the KDR-bioluminescence assay only detects VEGF activity. Unformulated growth factors and their combination were tested in the concentration range of $0-20 \mathrm{ng} / \mathrm{ml}$. Growth factor combinations achieved higher relative proliferations (up to 9-fold) compared to separate growth factors (up to 6-fold for FGF and 4-fold for VEGF), as shown in figure 9a. This result shows a strong proliferative 
effect of FGF and synergistic effect when VEGF and FGF were combined, in line with other studies. ${ }^{37,39}$ FGF showed a higher cell proliferation response than VEGF, also in line with previous studies. ${ }^{55,56}$ IGF (in yellow bars) did not induce endothelial cell proliferation in the concentrations tested, however, VEGF combined with IGF, or FGF combined with IGF showed increased cell proliferation and hence some potentiating effects of IGF (figure S3). A possible explanation for the absence of bioactivity of IGF is that the in vitro assay used is not suitable for determining IGF bioactivity, as recent studies demonstrated IGF's mitogenic properties by determining DNA levels in (tumor) cells. ${ }^{57,58}$ Although endothelial cell proliferation is an important aspect of angiogenesis, also other processes such as endothelial cell migration and tube formation play important roles in angiogenesis.

Although endothelial cell proliferation is an important aspect of angiogenesis, also other processes, such as endothelial cell migration and tube formation play important roles in angiogenesis and can be evaluated with in vitro assays. ${ }^{59}$

The relative cell proliferation of release samples of the microspheres loaded with VEGF, FGF and IGF is shown in figure 9b. At all tested time points, endothelial cell proliferation was significantly enhanced, ranging from 5-fold to 3-fold increased proliferation.

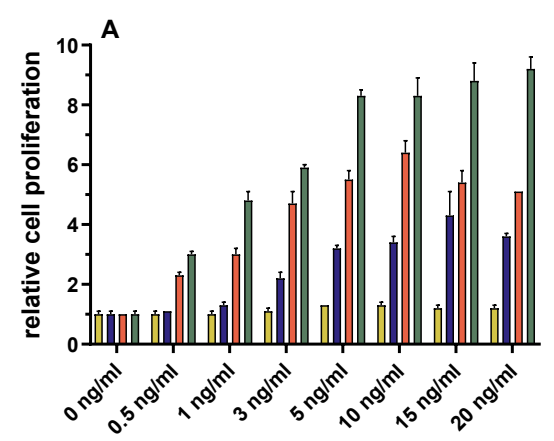

growth factor standards

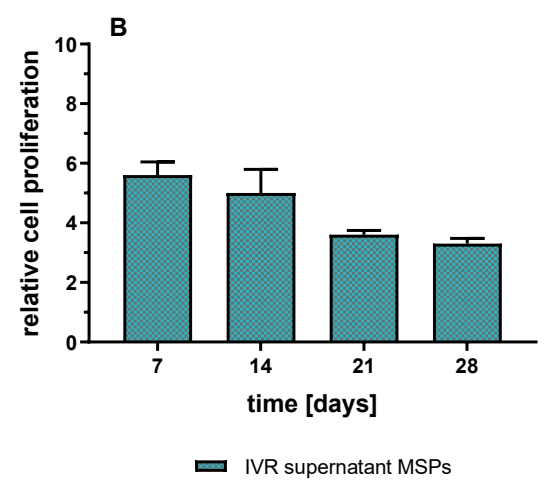

—IVR supernatant MSPs

$\square$ IGF $\square$ VEGF $\square$ FGF $\square$ VEGF+FGF+IGF (1:1:1)

FIGURE 9: Relative cell proliferation of growth factor standards and released growth factors was measured by Alamar Blue HUVEC proliferation assay. A: Relative cell proliferation of IGF (yellow bars), VEGF (blue bars), FGF (red bars) or a 1:1:1 combination of VEGF, FGF and IGF (green bars) in concentrations $0-20 \mathrm{ng} / \mathrm{ml}$. B: Relative cell proliferation of release media collected from $1 \mathrm{mg}$ microspheres loaded with VEGF, FGF and IGF using trapping agent HDS. IVR samples were diluted 500x. Bars represent average \pm SD $(n=3)$.

This demonstrates that in a time frame of 28 days, bioactive growth factors were continuously released from microspheres, in profiles and quantities that are optimal for a vascularization strategy. Current literature suggests that an average release rate of $150 \mathrm{ng}$ VEGF/day for four weeks is sufficient for vascularization of a $0.1 \mathrm{ml}$ biomaterial implant in rodents. ${ }^{17,} 18$ Based on the loading content of growth factors in microspheres (table 4), an estimate of $100 \mu \mathrm{g}$ microspheres co-loaded with VEGF, FGF and IGF will provide sufficient growth factor release 
(equations S1 and S2), considering the strong proliferative effect of FGF, the synergistic effect of VEGF and FGF, and IGF's reported mitogenic properties.

\section{Conclusions}

In this study, the suitability of active encapsulation (ASE) for loading a combination of growth factors in PLGA microspheres for sustained release was demonstrated. With this method, high loading efficiencies of VEGF, FGF and IGF were achieved using dextran sulfate as trapping agent. Bioactive growth factors were released continuously over a release period of four weeks, whereby a combination of growth factors had a stronger and complementary proliferative response than solely VEGF. Therefore, self-encapsulating microspheres offer sustained release of proangiogenic growth factors in their bioactive form in a time frame that is attractive for incorporation in biomaterial implants to achieve its functional vascularization.

\section{Acknowledgements}

This research is funded by European Union's Horizon 2020 research and innovation program under grant agreement number 645991 (DRIVE). Karina Scheiner received a personal travel grant from the Foundation "De Drie Lichten". The authors thank Linda Barthel (Microscope and Image Analysis Laboratory, University of Michigan, USA) for her excellent assistance with confocal fluorescence microscopy. Rae Sung Chang, Karl Olsen, Rose Ackermann (Department of Pharmaceutical Sciences and the Biointerfaces Institute, University of Michigan), Louis van Bloois, Sjaak Jong (Department of Pharmaceutics, Utrecht University, the Netherlands) and Gert Hendriks (InnoCore Pharmaceuticals BV, Groningen, the Netherlands) are greatly acknowledged for their contribution to this work. 


\section{References}

1. Goodnough, L. T.; Anderson, K. C. Recombinant growth factors. Transfus. Sci. 1995, 16, (1), 45-62.

2. Brizzi, M. F.; Tarone, G.; Defilippi, P. Extracellular matrix, integrins, and growth factors as tailors of the stem cell niche. Curr. Opin. Cell Biol. 2012, 24, (5), 645-651.

3. Gorelik, L.; Flavell, R. A. Transforming growth factor-beta in T-cell biology. Nat. Rev. Immunol. 2002, 2, (1), $46-53$.

4. Lee, K.; Silva, E. A.; Mooney, D. J. Growth factor delivery-based tissue engineering: general approaches and a review of recent developments. J R Soc Interface 2011, 8, (55), 153-70.

5. Novosel, E. C.; Kleinhans, C.; Kluger, P. J. Vascularization is the key challenge in tissue engineering. Adv Drug Deliv Rev 2011, 63, (4-5), 300-11.

6. Farina, M.; Alexander, J. F.; Thekkedath, U.; Ferrari, M.; Grattoni, A. Cell encapsulation: Overcoming barriers in cell transplantation in diabetes and beyond. Adv Drug Deliv Rev 2019, 139, 92-115.

7. Farina, M.; Chua, C. Y. X.; Ballerini, A.; Thekkedath, U.; Alexander, J. F.; Rhudy, J. R.; Torchio, G.; Fraga, D.; Pathak, R. R.; Villanueva, M.; Shin, C. S.; Niles, J. A.; Sesana, R.; Demarchi, D.; Sikora, A. G.; Acharya, G. S.; Gaber, A. O.; Nichols, J. E.; Grattoni, A. Transcutaneously refillable, 3D-printed biopolymeric encapsulation system for the transplantation of endocrine cells. Biomaterials 2018, 177, 125-138.

8. Rouwkema, J.; Rivron, N. C.; van Blitterswijk, C. A. Vascularization in tissue engineering. Trends Biotechnol. 2008, 26, (8), 434-41.

9. Wagner, E. R.; Parry, J.; Dadsetan, M.; Bravo, D.; Riester, S. M.; Van Wijnen, A. J.; Yaszemski, M. J.; Kakar, S. VEGFmediated angiogenesis and vascularization of a fumarate-crosslinked polycaprolactone (PCLF) scaffold. Connect. Tissue Res. 2018, 59, (6), 542-549.

10. Yla-Herttuala, S.; Rissanen, T. T.; Vajanto, I.; Hartikainen, J. Vascular endothelial growth factors: biology and current status of clinical applications in cardiovascular medicine. J. Am. Coll. Cardiol. 2007, 49, (10), 1015-26.

11. Said, S. S.; Pickering, J. G.; Mequanint, K. Advances in growth factor delivery for therapeutic angiogenesis. J. Vasc. Res. 2013, 50, (1), 35-51.

12. Presta, M.; Dell'Era, P.; Mitola, S.; Moroni, E.; Ronca, R.; Rusnati, M. Fibroblast growth factor/fibroblast growth factor receptor system in angiogenesis. Cytokine Growth Factor Rev. 2005, 16, (2), 159-78.

13. Bach, L. A. Endothelial cells and the IGF system. J. Mol. Endocrinol. 2015, 54, (1), R1-13.

14. Bowers, D. T.; Song, W.; Wang, L. H.; Ma, M. Engineering the vasculature for islet transplantation. Acta Biomater. $\mathbf{2 0 1 9}, 95,131-151$.

15. Smink, A. M.; Li, S.; Hertsig, D. T.; de Haan, B. J.; Schwab, L.; van Apeldoorn, A. A.; de Koning, E.; Faas, M. M.; Lakey, J. R.; de Vos, P. The efficacy of a prevascularized, retrievable poly(D,L,-lactide-co-epsilon-caprolactone) subcutaneous scaffold as transplantation site for pancreatic islets. Transplantation 2017, 101, (4), e112-e1 19.

16. Silva, E. A.; Mooney, D. J. Effects of VEGF temporal and spatial presentation on angiogenesis. Biomaterials 2010, $31,(6), 1235-41$.

17. Davies, N.; Dobner, S.; Bezuidenhout, D.; Schmidt, C.; Beck, M.; Zisch, A. H.; Zilla, P. The dosage dependence of VEGF stimulation on scaffold neovascularisation. Biomaterials 2008, 29, (26), 3531-8.

18. Amsden, B. G.; Timbart, L.; Marecak, D.; Chapanian, R.; Tse, M. Y.; Pang, S. C. VEGF-induced angiogenesis following localized delivery via injectable, low viscosity poly(trimethylene carbonate). J. Control. Release 2010, 145, (2), 109-15.

19. Cao, L.; Mooney, D. J. Spatiotemporal control over growth factor signaling for therapeutic neovascularization. Adv Drug Deliv Rev 2007, 59, (13), 1340-50.

20. Giteau, A.; Venier-Julienne, M. C.; Aubert-Pouessel, A.; Benoit, J. P. How to achieve sustained and complete protein release from PLGA-based microparticles? Int. J. Pharm. 2008, 350, (1-2), 14-26.

21. Ma, G. Microencapsulation of protein drugs for drug delivery: strategy, preparation, and applications. J. Control. Release 2014, 193, 324-40.

22. Iqbal, M.; Zafar, N.; Fessi, H.; Elaissari, A. Double emulsion solvent evaporation techniques used for drug encapsulation. Int. J. Pharm. 2015, 496, (2), 173-90.

23. Thomas, C. R.; Geer, D. Effects of shear on proteins in solution. Biotechnol. Lett. 2011, 33, (3), 443-56.

24. van de Weert, M.; Hoechstetter, J.; Hennink, W. E.; Crommelin, D. J. The effect of a water/organic solvent 
interface on the structural stability of lysozyme. J. Control. Release 2000, 68, (3), 351-9.

25. van de Weert, M.; Hennink, W. E.; Jiskoot, W. Protein instability in poly(lactic-co-glycolic acid) microparticles. Pharm. Res. 2000, 17, (10), 1159-67.

26. Desai, K. G.; Kadous, S.; Schwendeman, S. P. Gamma irradiation of active self-healing PLGA microspheres for efficient aqueous encapsulation of vaccine antigens. Pharm. Res. 2013, 30, (7), 1768-78.

27. Reinhold, S. E.; Desai, K. G.; Zhang, L.; Olsen, K. F.; Schwendeman, S. P. Self-healing microencapsulation of biomacromolecules without organic solvents. Angew. Chem. Int. Ed. Engl. 2012, 51, (43), 10800-3.

28. Shah, R. B.; Schwendeman, S. P. A biomimetic approach to active self-microencapsulation of proteins in PLGA. J. Control. Release 2014, 196, 60-70.

29. Fu, K.; Pack, D. W.; Klibanov, A. M.; Langer, R. Visual evidence of acidic environment within degrading poly(lacticco-glycolic acid) (PLGA) microspheres. Pharm. Res. 2000, 17, (1), 100-6.

30. Li, L.; Schwendeman, S. P. Mapping neutral microclimate pH in PLGA microspheres. J. Control. Release 2005, 101, (1-3), 163-73.

31. Giteau, A.; Venier-Julienne, M. C.; Marchal, S.; Courthaudon, J. L.; Sergent, M.; Montero-Menei, C.; Verdier, J. M.; Benoit, J. P. Reversible protein precipitation to ensure stability during encapsulation within PLGA microspheres. Eur. J. Pharm. Biopharm. 2008, 70, (1), 127-36.

32. Estey, T.; Kang, J.; Schwendeman, S. P.; Carpenter, J. F. BSA degradation under acidic conditions: a model for protein instability during release from PLGA delivery systems. J. Pharm. Sci. 2006, 95, (7), 1626-39.

33. Zhu, G.; Mallery, S. R.; Schwendeman, S. P. Stabilization of proteins encapsulated in injectable poly (lactide- coglycolide). Nat. Biotechnol. 2000, 18, (1), 52-7.

34. van der Walle, C. F.; Sharma, G.; Ravi Kumar, M. Current approaches to stabilising and analysing proteins during microencapsulation in PLGA. Expert Opin Drug Deliv 2009, 6, (2), 177-86.

35. Schwendeman, S.P. Recent advances in the stabilization of proteins encapsulated in injectable PLGA delivery systems. Crit. Rev. Ther. Drug Carrier Syst. 2002, 19, (1), 73-98.

36. Wetter, L. R.; Deutsch, H. F. Immunological studies on egg white proteins. IV. Immunochemical and physical studies of lysozyme. J. Biol. Chem. 1951, 192, (1), 237-42.

37. Kim, S. K.; Lee, J.; Song, M.; Kim, M.; Hwang, S. J.; Jang, H.; Park, Y. Combination of three angiogenic growth factors has synergistic effects on sprouting of endothelial cell/mesenchymal stem cell-based spheroids in a 3D matrix. J. Biomed. Mater. Res. B Appl. Biomater. 2016, 104, (8), 1535-1543.

38. Liu, G.; Pareta, R. A.; Wu, R.; Shi, Y.; Zhou, X.; Liu, H.; Deng, C.; Sun, X.; Atala, A.; Opara, E. C.; Zhang, Y. Skeletal myogenic differentiation of urine-derived stem cells and angiogenesis using microbeads loaded with growth factors. Biomaterials 2013, 34, (4), 1311-26.

39. Cittadini, A.; Monti, M. G.; Petrillo, V.; Esposito, G.; Imparato, G.; Luciani, A.; Urciuolo, F.; Bobbio, E.; Natale, C. F.; Sacca, L.; Netti, P. A. Complementary therapeutic effects of dual delivery of insulin-like growth factor-1 and vascular endothelial growth factor by gelatin microspheres in experimental heart failure. Eur. J. Heart Fail. 2011, $13,(12), 1264-74$.

40. Ferrara, N.; Leung, D. W.; Cachianes, G.; Winer, J.; Henzel, W. J. Purification and cloning of vascular endothelial growth factor secreted by pituitary folliculostellate cells. Methods Enzymol. 1991, 198, 391-405.

41. Tabata, Y.; Yamada, K.; Miyamoto, S.; Nagata, I.; Kikuchi, H.; Aoyama, I.; Tamura, M.; Ikada, Y. Bone regeneration by basic fibroblast growth factor complexed with biodegradable hydrogels. Biomaterials 1998, 19, (7-9), 807-815.

42. Verschure, P. J.; Van Noorden, C. J.; Van Marle, J.; Van den Berg, W. B. Articular cartilage destruction in experimental inflammatory arthritis: insulin-like growth factor-1 regulation of proteoglycan metabolism in chondrocytes. Histochem. J. 1996, 28, (12), 835-57.

43. Scheiner, K. C.; Maas-Bakker, R. F.; Nguyen, T. T.; Duarte, A. M.; Hendriks, G.; Sequeira, L.; Duffy, G. P.; Steendam, R.; Hennink, W. E.; Kok, R. J. Sustained release of vascular endothelial growth factor from poly(epsiloncaprolactone-PEG-epsilon-caprolactone)-b-poly(l-lactide) multiblock copolymer microspheres. ACS Omega 2019, 4, (7), 11481-11492.

44. Wang, L.; Xu, G. L.; Gao, K.; Wilkinson, J.; Zhang, F.; Yu, L.; Liu, C. Y.; Yu, C. F.; Wang, W. B.; Li, M.; Chen, W.; Fan, F.; Cong, M.; Wang, J. Z. Development of a robust reporter-based assay for the bioactivity determination of antiVEGF therapeutic antibodies. J. Pharm. Biomed. Anal. 2016, 125, 212-8. 
45. Fonte, P.; Araujo, F.; Seabra, V.; Reis, S.; van de Weert, M.; Sarmento, B. Co-encapsulation of lyoprotectants improves the stability of protein-loaded PLGA nanoparticles upon lyophilization. Int. J. Pharm. 2015, 496, (2), 850-62.

46. Passerini, N.; Craig, D. Q. M. An investigation into the effects of residual water on the glass transition temperature of polylactide microspheres using modulated temperature DSC. J. Control. Release 2001, 73, (1), 111-115.

47. Shah, S. S.; Cha, Y.; Pitt, C. G. Poly (glycolic acid-co-dl-lactic acid): diffusion or degradation controlled drug delivery? J. Control. Release 1992, 18, (3), 261-270.

48. Zhao, W.; McCallum, S. A.; Xiao, Z.; Zhang, F.; Linhardt, R. J. Binding affinities of vascular endothelial growth factor (VEGF) for heparin-derived oligosaccharides. Biosci. Rep. 2012, 32, (1), 71-81.

49. Water, J. J.; Schack, M. M.; Velazquez-Campoy, A.; Maltesen, M. J.; van de Weert, M.; Jorgensen, L. Complex coacervates of hyaluronic acid and lysozyme: effect on protein structure and physical stability. Eur. J. Pharm. Biopharm. 2014, 88, (2), 325-31.

50. Samadi, N.; Abbadessa, A.; Di Stefano, A.; van Nostrum, C. F.; Vermonden, T.; Rahimian, S.; Teunissen, E. A.; van Steenbergen, M. J.; Amidi, M.; Hennink, W. E. The effect of lauryl capping group on protein release and degradation of poly(D,L-lactic-co-glycolic acid) particles. J. Control. Release 2013, 172, (2), 436-43.

51. Fredenberg, S.; Wahlgren, M.; Reslow, M.; Axelsson, A. The mechanisms of drug release in poly(lactic-co-glycolic acid)-based drug delivery systems--a review. Int. J. Pharm. 2011, 415, (1-2), 34-52.

52. Li, S. Hydrolytic degradation characteristics of aliphatic polyesters derived from lactic and glycolic acids. J. Biomed. Mater. Res. 1999, 48, (3), 342-53.

53. Ritger, P. L.; Peppas, N. A. A simple equation for description of solute release II. Fickian and anomalous release from swellable devices. J. Control. Release 1987, 5, 37 - 42.

54. Allison, S. D. Analysis of initial burst in PLGA microparticles. Expert Opin Drug Deliv 2008, 5, (6), 615-28.

55. Yoshida, A.; Anand-apte, B.; Zetter, B. R. Differential endothelial migration and proliferation to basic fibroblast growth factor and vascular endothelial growth factor. Growth Factors 2009, 13, (1-2), 57-64.

56. Zieris, A.; Prokoph, S.; Levental, K. R.; Welzel, P. B.; Grimmer, M.; Freudenberg, U.; Werner, C. FGF-2 and VEGF functionalization of starPEG-heparin hydrogels to modulate biomolecular and physical cues of angiogenesis. Biomaterials 2010, 31, (31), 7985-94.

57. Hameed, A.; Gallagher, L. B.; Dolan, E.; O'Sullivan, J.; Ruiz-Hernandez, E.; Duffy, G. P.; Kelly, H. Insulin-like growth factor-1 (IGF-1) poly (lactic-co-glycolic acid) (PLGA) microparticles - development, characterisation, and in vitro assessment of bioactivity for cardiac applications. J. Microencapsul. 2019, 1-11.

58. Clark, A.; Milbrandt, T. A.; Hilt, J. Z;; Puleo, D. A. Retention of insulin-like growth factor I bioactivity during the fabrication of sintered polymeric scaffolds. Biomed Mater 2014, 9, (2), 025015.

59. Auerbach, R.; Lewis, R.; Shinners, B.; Kubai, L.; Akhtar, N. Angiogenesis assays: a critical overview. Clin. Chem. 2003, 49, (1), 32-40. 


\section{Supporting Information}

A

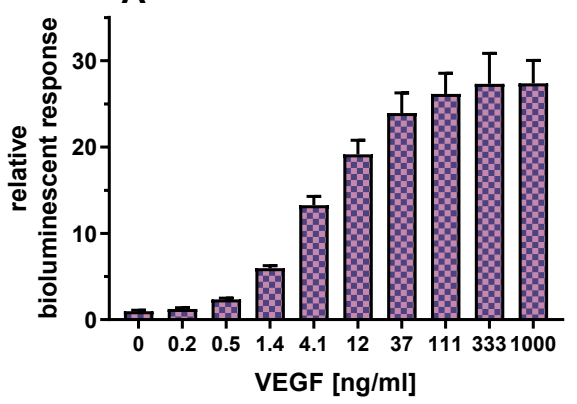

B

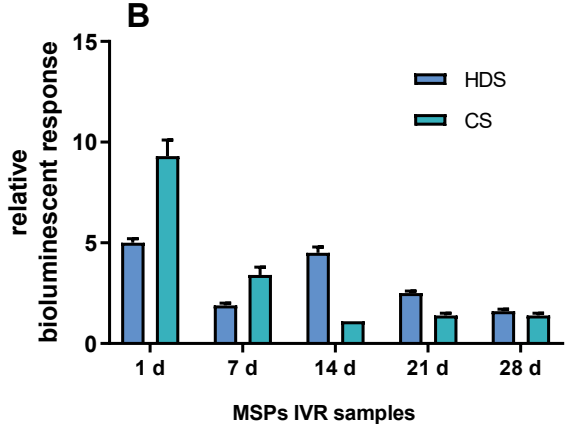

FIGURE S1: Relative bioluminescent response of VEGF standards $0-1000 \mathrm{ng} / \mathrm{ml}$ measured by Promega VEGF bioreceptor assay with KDR/NFAT-RE HEK 293 cells. Bars represent average \pm SD $(n=3)$.

\section{Determination of growth factor loading by SE-UPLC}

Release samples were analyzed for their growth factor content using size exclusion ultra high performance liquid chromatography (SE-UPLC, Waters Acquity H-class UPLC system (Waters, Milford, USA)) as described previously.' In short, $7.5 \mu$ samples were injected onto a Waters Acquity UPLC Protein BEH SEC 200 column (Waters, Milford, USA) at a flow rate of using 0.1 M sodium phosphate, 0.3 M sodium sulfate pH 6.7 as mobile phase. VEGF, FGF and IGF were detected at $214 \mathrm{~nm}$ at $\sim 5.5$ minutes (VEGF), $\sim 6$ minutes (FGF) and $\sim 7$ minutes (IGF).

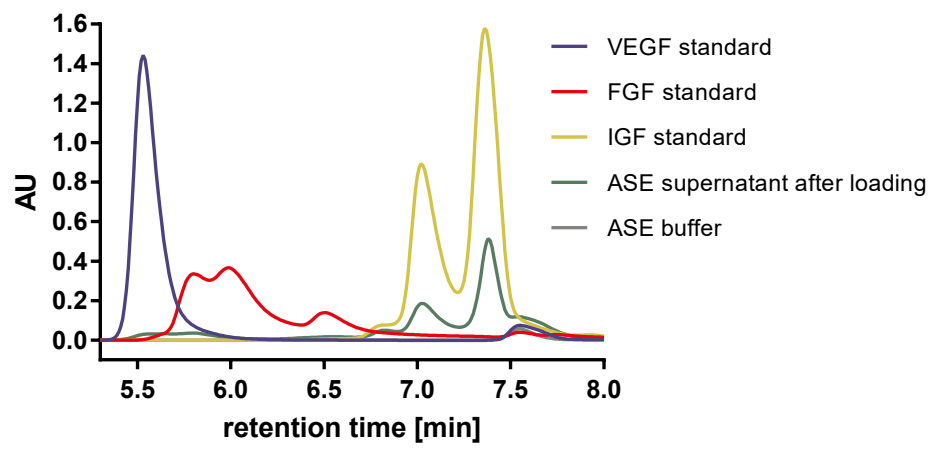

FIGURE S2: SE-UPLC chromatograms of medium after ASE loading of a combination of VEGF, FGF and IGF (ASE supernatant after loading), growth factor standards (VEGF, FGF, IGF each $333 \mathrm{ug} / \mathrm{ml}$ ) and ASE buffer. Retention time VEGF: 5.5 minutes, FGF: 5.8 minutes, 6.5 minutes, IGF: 7.0 minutes, 7.4 minutes. Please note that ASE supernatant after loading clearly shows peaks corresponding to IGF (equivalent to $25 \%$ non-loaded IGF) while VEGF and FGF peaks are absent. 


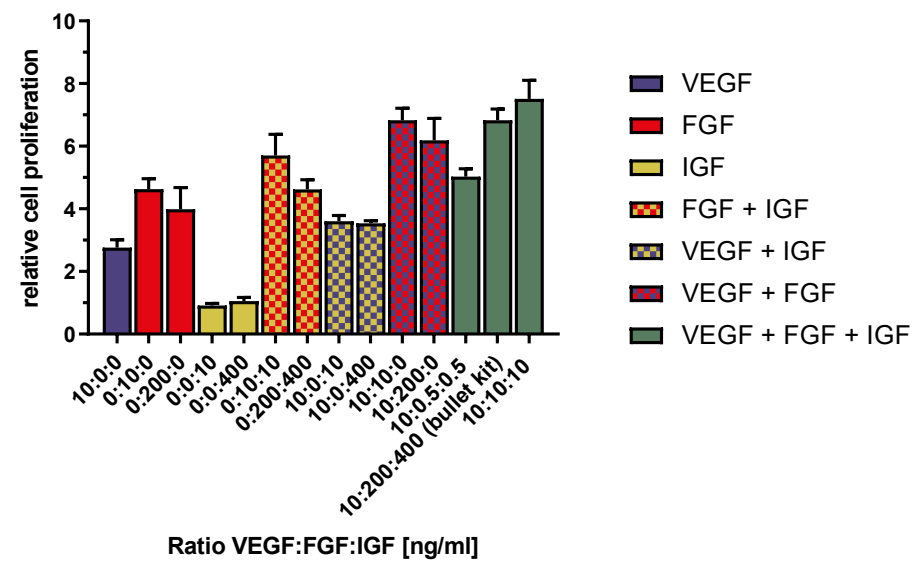

FIGURE S3: Relative cell proliferation of different combinations of VEGF, FGF and/or IGF at two ratios, i.e. 1:1:1 ratio and 1:20:40 weight ratio, determined by Alamar Blue proliferation assay with HUVECs. Ratio 1:20:40 was selected based on growth factor supplements included in the HUVEC cell culture medium (1:20:40 VEGF:FGF:IGF).

\section{Estimation of the amount of microspheres co-loaded with VEGF, FGF and IGF required for vascularization of a biomaterial implant}

$$
150 \mathrm{ng} V E G F / \text { day } \times 28 \text { days }=4200 \mathrm{ng} \operatorname{VEGF}(4.2 \mu \mathrm{g})
$$

EQUATION S1: Amount of VEGF release required for vascularization of a $0.1 \mathrm{ml}$ biomaterial implant.,

$$
4.3 w t \% \times 100 \mu \mathrm{g} \text { microspheres }=4.3 \mu \mathrm{g} \text { loaded growth factors }
$$

EQUATION S2: Amount of loaded growth factors in $100 \mu \mathrm{g}$ microspheres based on a measured 4.3 wt\% loading content (reported in table IV). Considerations for an accurate estimate include an observed higher proliferative response for FGF compared to VEGF (and IGF), as well as an absence of IGF bioactivity in the assay used in this study. 


\section{References}

1. Shah, R.B.; Schwendeman S.P. A biomimetic approach to active self-microencapsulation of proteins in PLGA. J. Control. Release 2014, 196, 60-70.

2. Amsden, B.G.; Timbart, L.; Marecak, D.; Chapanian, R.; Tse, M.Y.; Pang, S.C. VEGF-induced angiogenesis following localized delivery via injectable, low viscosity poly(trimethylene carbonate). J. Control. Release 2010, 145, (2), 109-15.

3. Davies, N.; Dobner, S.; Bezuidenhout, D.; Schmidt, C.; Beck, M.; Zisch, P. The dosage dependence of VEGF stimulation on scaffold neovascularization. Biomaterials 2008, 29, (26), 3531-8.

\section{Web references}

aavailable from: https://www.promocell.com/f/product-information/manual/C-39216.pdf (accessed 19 ${ }^{\text {th }}$ September 2019). 


\section{Chapter 5}

\section{Sustained Release of Vascular Endothelial Growth Factor from Chondroitin Sulfate-based Hydrogels}

Karina C. Scheiner', Carl C.L. Schuurmans ${ }^{1,2}$, Roderick van den Berg', Manavi Kapur', Tina Vermonden', Wim E. Hennink', Robbert ]. Kok' 


\section{Abstract}

Methacrylated chondroitin sulfate (CSMA)-based hydrogels are attractive scaffolds for tissue-engineering applications due to their tailorable mechanical properties and good biocompatibility. Because of the negatively charged carboxylic and sulfate groups, chondroitin sulfate is able to ionically interact at neutral $\mathrm{pH}$ with positively charged proteins, such as lysozyme (LYZ) and growth factors with a basic pl, also named complex precipitates. In the present study it was explored whether CSMA hydrogels complexed with LYZ can be used for the sustained release of vascular endothelial growth factor (VEGF), hypothesizing that these CSMA-lysozyme complex precipitates modulate the release of VEGF. VEGF-loaded depot systems as integral part of tissue-engineered constructs can be used for local release of this growth factor, with the aim of stimulating blood vessel growth (vascularization) towards such implants. In this study, VEGF was post-loaded in CSMA hydrogels (obtained by UV-curing of an aqueous solution of CSMA) by ionic interaction in a low ionic strength buffer ( $20 \mathrm{mM}$ HEPES $\mathrm{pH}$ 7.4) and subsequently further loaded with an excess of LYZ. The VEGF loading efficiency was 65\% VEGF whereas a slightly lower loading efficiency (54\%) was found when VEGF loading was followed by incubation of the CSMA hydrogel with LYZ ( 40\% LYZ loading efficiency). VEGF was not loaded when VEGF and LYZ were simultaneously present in the loading buffer, indicating an outcompeting effect of LYZ due to its huge molar excess (>600 times) and stronger complexation due to its higher isoelectric point (11.4 of LYZ versus 8.5 of VEGF). VEGFloaded CSMA hydrogels without LYZ loading showed extensive swelling during the four-week incubation in phosphate buffered saline at $37^{\circ} \mathrm{C}$, in which the swelling ratio increased eightfold before dissolution of the hydrogel due to hydrolysis of the ester bonds in the crosslinks of the hydrogels. LYZ-loaded hydrogels (forming LYZ-CSMA complex precipitates) did not show the distinctive swelling behavior of CSMA hydrogels, leading to a slightly longer degradation time of six weeks. In vitro release studies in phosphate buffered saline (physiological ionic strength) showed that VEGF was continuously released for four weeks when loaded solely in hydrogels, reaching 60\% cumulative release as determined by SE-UPLC. VEGF was released from LYZ-CSMA complex-precipitate hydrogels for an additional two weeks, reaching 40\% cumulative release after six weeks. The delaying effect of LYZ-CSMA complexes on VEGF release can be explained by a lower swelling, leading to a lower diffusivity of VEGF in the hydrogel matrix. However, more research has to be done for understanding the underlying mechanisms of VEGF release from LYZ-CSMA complex precipitate-containing hydrogels, and degradation of the CSMA hydrogel network. In conclusion, our results demonstrate that postloading of VEGF in CSMA hydrogels is a feasible approach for achieving sustained release of VEGF, and that LYZ-CSMA complex precipitates prolong the release window of VEGF from CSMA hydrogels. 


\section{Introduction}

Tissue-engineering is a relatively new research field aiming the repair or replacement of damaged and/or non-functional tissues.1, 2 Commonly, a functional tissue-engineered construct is composed of a biomaterial scaffold, which can be loaded with cells or organoids and drugs or biotherapeutic molecules like growth factors that support the function of the engineered material. ${ }^{3}$ The scaffold provides mechanical support and a bio-friendly environment for embedded cells. ${ }^{4,5}$ Bioactive compounds can be incorporated in the scaffold to inhibit the immunological response to the bioartificial construct ${ }^{6}$ or to induce blood vessel growth towards the scaffold (vascularization), thereby supplying the embedded cells with nutrients and oxygen and allowing removal of waste products. ${ }^{7}$

Hydrogels are attractive scaffold materials for the development of tissue-engineering constructs and consists of hydrophilic crosslinked polymer chains that retain high amounts of water., ${ }^{3,-10}$ Glycosaminoglycans, such as heparin, hyaluronic acid and chondroitin sulfate, as natural polymers are frequently exploited for the design of hydrogels used for the design of tissue engineering constructs.1"12 These linear anionic polysaccharides are extracellular components in many tissues. Chondroitin sulfate is a prominent glycosaminoglycan composed of repeating disaccharide units D-glucuronic acid and sulfated N-acetyl-Dgalactosamine (figure 1, in black and blue). ${ }^{13}$ The carboxyl group of CS can be functionalized with glycidyl methacrylate, forming ester-linked methacrylated chondroitin sulfate (CSMA, figure 1), which can be photo-crosslinked to obtain biodegradable CS hydrogel networks with tailorable mechanical strength and stiffness. ${ }^{14,15}$

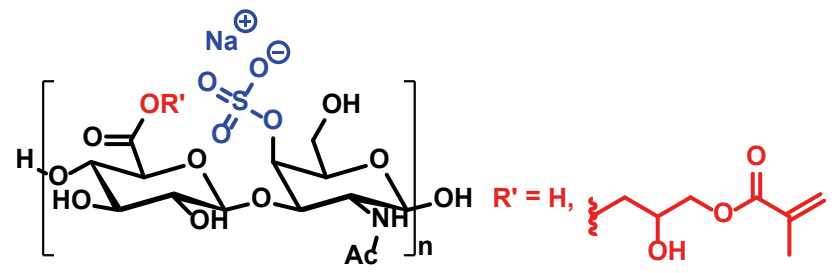

FIGURE 1: Chemical structure of methacrylated chondroitin sulfate (CSMA).

Due to the presence of carboxylic and sulfate groups, chondroitin sulfate is negatively charged at physiological pH and can therefore interact and form complexes with positively charged proteins, such as growth factors of high $\mathrm{pl}^{16-18}$. Chondroitin sulfate, and other glycosaminoglycans, play a major role in the sequestration, regulation and inter-cellular transport of growth factors. ${ }^{13}$ Furthermore, the complexation of chondroitin sulfate with positively charged lysozyme (LYZ), a protein of $14 \mathrm{kDa}$ and an isoelectric point of $11.4^{19}$, leads to the formation of liquid-solid phase-separated complexes (also referred to as complex precipitates), ${ }^{17}, 20$ which are characterized by the tight binding of the oppositely 
charged proteins to the negatively charged biopolymers and expulsion of counterions and water molecules. ${ }^{20}$ Complex precipitates of glycosaminoglycans and LYZ slowly dissociate (depending on the $\mathrm{pH}$ and ionic strength of the medium), resulting in sustained release of LYZ from crosslinked CS matrices. ${ }^{17,21}$ Therefore, these matrices are attractive systems to develop sustained release formulations of vascular endothelial growth factor (VEGF), a potent and wellcharacterized proangiogenic growth factor. ${ }^{22,23}$ In this context, we studied how LYZ-CSMA precipitates (through the addition of an excess amount of LYZ) modulated the VEGF release.

In this study, we aimed to obtain sustained release formulation of VEGF exploiting LYZ - CSMA complex precipitate - hydrogels with a target release time frame of four weeks, which has shown to be beneficial functional vascularization of tissue-engineering constructs. ${ }^{24-27}$ We evaluated the loading of positively charged VEGF (isoelectric point of $8.5^{28}$ ) in CSMA hydrogels via ionic interaction in low ionic strength buffer (figure 2a). Further, we investigated the effect of coloading an excess LYZ in the VEGF-loaded hydrogels - thus forming complex precipitates of LYZ in the CSMA hydrogel - as an additional measure of modulating VEGF release (figure 2b).
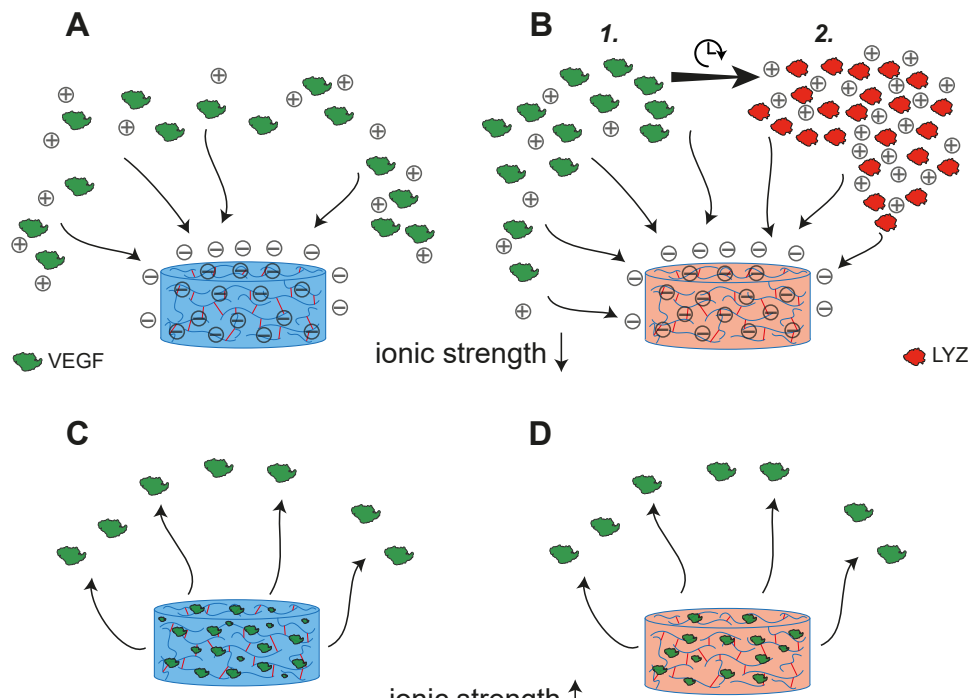

ionic strength $\uparrow$

D

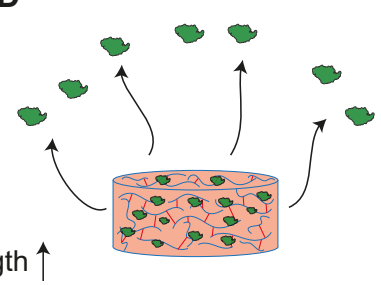

FIGURE 2: Schematic image the study design. CSMA hydrogels in blue (red: crosslinking by methacrylate), VEGF (green), lysozyme (LYZ, in red). A: Loading VEGF in CSMA hydrogels in low ionic strength buffer through ionic interaction. B: Loading of VEGF in CSMA hydrogels through ionic interaction and subsequent loading of LYZ, both in low ionic strength buffer. The release of VEGF (and LYZ) from hydrogels (C: VEGF-loaded hydrogels, D: VEGF- and LYZloaded hydrogels) in a buffer with a higher ionic strength than the loading buffer. 


\section{Experimental section}

\section{Materials}

Chondroitin sulfate type A sodium salt (from bovine trachea), tetrabutylammonium (TBA), glycidyl methacrylate (GMA), 4-dimethylaminopyridine (DMAP), lysozyme from chicken egg white, HEPES, Tween 20 , sodium azide, sodium sulfate $\left(\mathrm{Na}_{2} \mathrm{SO}_{4}\right)$ and sodium phosphate monobasic monohydrate $\left(\mathrm{NaH}_{2} \mathrm{PO}_{4} . \mathrm{H}_{2} \mathrm{O}\right)$ were obtained from Sigma Aldrich (Zwijndrecht, the Netherlands). Recombinant human VEGF ${ }_{165}$ was obtained from PeproTech Inc. (Rocky Hill, NJ, USA). Gibco Dulbecco's Phosphate Buffered Saline (DPBS, 10x, composition $27 \mathrm{mM} \mathrm{KCl}, 15$ $\mathrm{mM} \mathrm{H}_{2} \mathrm{PO}_{4^{\prime}} 1.4 \mathrm{M} \mathrm{NaCl}, 81 \mathrm{mM} \mathrm{Na}_{2} \mathrm{HPO}_{4} .7 \mathrm{H}_{2} \mathrm{O}$ ) was purchased from Thermo Fisher Scientific (the Netherlands). Lithium phenyl-2,4,6-trimethylbenzoylphosphinate (LAP) was obtained from Tokyo Chemical Industry (Tokyo, Japan).

\section{Methods}

\section{Synthesis and characterization of CSMA}

Methacrylated chondroitin sulfate (CSMA) with a 15\% degree of methacrylation (figure 1) was synthesized and characterized as previously reported by Abbadessa et al. ${ }^{29}$ In brief, sodium salt of chondroitin sulfate was exchanged by tetrabutylammonium (TBA) using ion exchange chromatography. Chondroitin sulfate-TBA dissolved in DMSO (27 mg/ml; $7.175 \mathrm{~g}$ CS-TBA in $265 \mathrm{ml}$ DMSO) was then reacted with $790 \mu \mathrm{l}$ glycidyl methacrylate (GMA) using 4-dimethylaminopyridine (DMAP) as catalyst in DMSO. Finally, the formed CSMA was first dialyzed against $150 \mathrm{mM} \mathrm{NaCl}$ for 4 days, and subsequently against distilled water for 3 days and finally freeze-dried overnight. The degree of methacrylation of freeze-dried CSMA was determined by integration of ${ }^{1} \mathrm{H}$-NMR signals of the 2 protons of the vinyl group of the methacrylate versus the proton of the acetyl group of chondroitin carbohydrate unit (equation $1,{ }^{1} \mathrm{H}-\mathrm{NMR}$ spectrum shown in figure $\left.\mathrm{S} 1\right)^{29}$

$$
D M[\%]=\frac{\operatorname{average}\left(I_{6.20}, I_{5.77}\right)}{\left(I_{2.18-1.86}-3\right) / 3} \times 100
$$

EQUATION 1: Calculation of the degree of methacrylation (DM)

\section{Preparation of CSMA hydrogels}

CSMA hydrogel disks were prepared as follows. Freeze-dried CSMA was dissolved in water to obtain a 15 wt\% solution (target volume: $850 \mu \mathrm{l}$ ). Next, $50 \mu \mathrm{l}$ of a $1 \mathrm{wt} \%$ lithium phenyl-2,4,6trimethylbenzoylphosphinate (LAP) solution in water was added to the CSMA solution and gently mixed. The obtained solution was then pipetted into a Teflon mold (dimensions of the mold: diameter $6 \mathrm{~mm}$, height $2 \mathrm{~mm}$ ). The mold was exposed to UV light for 900 seconds at a 
distance of $5 \mathrm{~cm}$ using a Bluepoint 4 UV lamp (point light source, wavelength range: 300 - 600 $\mathrm{nm}$, intensity at $5 \mathrm{~cm}: 80 \mathrm{~mW} / \mathrm{cm}^{2}$, Honle UVTechnology AG, Gräfelfing, Germany). Afterwards, the hydrogels were removed and transferred into $3 \mathrm{ml}$ glass snap bottles (previously weighed). The hydrogel-containing vial was weighed and imaged.

\section{Loading of VEGF and/or LYZ in CSMA hydrogels}

The obtained hydrogels were incubated in $1 \mathrm{ml}$ of $20 \mathrm{mM}$ HEPES pH 7.4 for 3 hours to allow for swelling at room temperature. Afterwards, the external liquid was removed, the hydrogel was imaged and the wet weight of the hydrogel was noted.

Next, $1 \mathrm{ml}$ of VEGF loading solutions $(250 \mu \mathrm{g} / \mathrm{ml})$ in $20 \mathrm{mM}$ HEPES of pH 7.4 was added to the hydrogels. For some hydrogels, the loading solution was enriched with LYZ (60 mg in $1 \mathrm{ml} 20$ mM HEPES (pH 7.4)). Hydrogels to which $1 \mathrm{ml}$ of a $60 \mathrm{mg} / \mathrm{ml}$ LYZ solution in $20 \mathrm{mM}$ HEPES pH 7.4 or $1 \mathrm{ml}$ of $20 \mathrm{mM}$ HEPES pH 7.4 were added served as controls.

Hydrogels were subsequently incubated for 24 hours at room temperature. Next, the protein solution was removed and when applicable, VEGF-loaded hydrogels were incubated for another 24 hours with a LYZ solution (1 ml of a 60 mg/ml LYZ solution in 20 mM HEPES (pH 7.4)).

The concentrations of VEGF and/or LYZ in the loading solutions after incubation with the hydrogels were analyzed by SE-UPLC, as described in section Quantification of VEGF and LYZ by SE-UPLC. VEGF loading content (wt \%) and VEGF/LYZ loading efficiency [\%] were calculated according to equations 2 and 3. Protein-loaded hydrogels were washed once with $1 \mathrm{ml}$ of 20 mM HEPES pH 7.4 and imaged. The weight of the hydrogels was noted to calculate the swelling ratio based on the weight of the hydrogel after UV-curing. The diameter is the hydrogels after UV curing, after swelling in HEPES buffer and protein loading was determined with ImageJ (NIH, Bethesda, MD, USA).

$$
\text { Loading }[w t \%]=\frac{\text { loaded protein }[\text { weight }]}{\text { CSMAin hydrogel }[\text { dryweight }]+\text { loaded protein }[\text { weight }]} \times 100 \%
$$

EQUATION 2: Calculation of the VEGF loading content [wt \%]

$$
\text { Loading efficiency }[\%]=\frac{\text { loaded } \text { protein }[\text { weight }]}{\text { added protein }[\text { weight }]} \times 100 \%
$$

EQUATION 3: Calculation of the VEGF/LYZ loading efficiency [\%]

\section{Release of VEGF and/or LYZ from hydrogels}

Protein-loaded hydrogels were incubated at $37^{\circ} \mathrm{C}$ in $1 \mathrm{ml}$ of in vitro release (IVR) buffer consisting of Dulbecco's PBS (supplemented with $0.025 \%$ Tween 20 and $0.02 \% \mathrm{NaN}_{3}(\mathrm{pH} 7.4)$ ) or $500 \mathrm{mM} \mathrm{NaCl}, 20 \mathrm{mM}$ HEPES pH 7.4. At different time points, the supernatants were collected 
and the wet-weight of the hydrogels was determined. Next, $800 \mu$ l of supernatant was added back into the vials together with $200 \mu$ of fresh buffer. The collected release samples (200 $\mu$ l) were analyzed by SE-UPLC for VEGF and/or LYZ content, as described in section Quantification of VEGF and LYZ by SE-UPLC. Cumulative release data were fitted using the Korsmeyer-Peppas model ${ }^{30}$ (equation 3), in which $Q_{t}$ is the amount of protein released at time $t, Q_{0}$ is the initial amount of protein in hydrogels, $n$ is the diffusional exponent indicative of the transport mechanism, and $K_{p}$ is the Korsmeyer-Peppas constant incorporating structural and geometric characteristics of the hydrogel (equation 3). ${ }^{30}$ The diffusional exponent $n$ was calculated from the fitted linear regression lines of log (\% drug released) versus log (time) (equation 4).

$$
\frac{Q_{t}}{Q_{0}}=K_{p} \times t^{n}
$$

EQUATION 3: Korsmeyer-Peppas equation

$$
\log \left(\frac{Q_{t}}{Q_{0}}\right)=\log \left(K_{p}\right)+n \log (t)
$$

EQUATION 4: Adapted Korsmeyer-Peppas equation

\section{Protein release in low or high ionic strength buffers}

Protein-loaded hydrogels were incubated either in low ionic strength buffer ( $1 \mathrm{ml}$ of $20 \mathrm{mM}$ HEPES pH 7.4) or high ionic strength buffer (1 $\mathrm{ml}$ of $500 \mathrm{mM} \mathrm{NaCl}, 20 \mathrm{mM} \mathrm{HEPES} \mathrm{pH} \mathrm{7.4)}$ at $37^{\circ} \mathrm{C}$. At different time points, release samples were collected and analyzed by SE-UPLC measurements (section Quantification of VEGF and LYZ by SE-UPLC) and also the wet-weights of the hydrogels were determined.

\section{Quantification of VEGF and LYZ by SE-UPLC}

VEGF and LYZ content in loading and release samples were determined by size exclusion UPLC (SE-UPLC) using a previously reported method. ${ }^{31}$ In brief, $7.5 \mu$ l aliquots of samples and standards were injected into a Waters Acquity H-class UPLC system (Waters, Milford, USA) equipped with a Waters Acquity UPLC protein BEH SEC column maintained at room temperature $\left(21^{\circ} \mathrm{C}\right)$. The mobile phase consisted of $0.1 \mathrm{M}$ sodium phosphate and $0.3 \mathrm{M}$ sodium sulfate of $\mathrm{pH}$ 6.7. VEGF or LYZ standards were prepared in $20 \mathrm{mM} \mathrm{HEPES} \mathrm{pH} \mathrm{7.4} \mathrm{or} \mathrm{IVR}$ buffer, in concentrations 0 - $250 \mu \mathrm{g} / \mathrm{ml}$ (VEGF) or 0 - $2000 \mu \mathrm{g} / \mathrm{ml}$ (LYZ). VEGF samples were injected without dilution, LYZ samples were diluted 50x prior to injection. VEGF and LYZ were detected by UV at $214 \mathrm{~nm}$ at $\sim 5$ minutes (VEGF) and $\sim 7$ minutes (LYZ) retention time (see figures S2, S3 and S4). 


\section{Statistical analysis}

Statistical analysis was performed with GraphPad prism7 using the paired t-test. Differences between the analyzed groups were considered significant if $p<0.05$.

\section{Results and Discussion}

In this study, VEGF (pl: 8.528) was loaded in CSMA hydrogels through ionic interaction by incubation in low ionic strength buffer (20 mM HEPES pH 7.4). VEGF-loaded hydrogels were subsequently incubated in a concentrated LYZ solution to obtain LYZ-CSMA complex precipitates. Three formulations were prepared, i.e. CSMA hydrogels loaded solely with VEGF, VEGF loaded followed by LYZ loading (VEGF / LYZ), or CSMA hydrogels simultaneously incubated with VEGF and LYZ (VEGF + LYZ). CSMA hydrogels were also loaded solely with LYZ (LYZ). The loading content of VEGF and loading efficiencies of VEGF and LYZ are reported in table 1. When VEGF was loaded in the CSMA hydrogels, a $65 \%$ loading efficiency was measured (1.8 wt\% loading). It is likely that incomplete loading was due to relatively low overall positive charge density since VEGF's isoelectric point $\left(8.5^{28}\right)$ is close to the $\mathrm{pH}$ of the loading solution (7.4). A slightly lower VEGF loading efficiency, i.e. 54\%, was measured when VEGF loading was followed by incubation of an excess of lysozyme, indicating that part of the VEGF was displaced by LYZ in the second incubation step (i.e. $11 \%$ ) due to a higher pl of the LYZ $\left(11.4^{19}\right.$, compared to the pl of VEGF) and thus stronger interaction with CSMA. On the other hand, no VEGF was loaded when VEGF was co-incubated with LYZ. Likely, under these conditions, binding of VEGF by the hydrogel was outcompeted by the high concentration of $L Y Z$ and the higher overall positive charge of the latter protein $(250 \mu \mathrm{g} / \mathrm{ml} \mathrm{VEGF} \mathrm{versus} 60$ $\mathrm{mg} / \mathrm{ml} \mathrm{LYZ} ; 1: 612 \mathrm{~mol} / \mathrm{mol}$ ratio, pl LYZ: 11.4 $\left.{ }^{19}\right)$. The LYZ loading efficiency in hydrogels ranged from $\sim 36$ to $\sim 47 \%$ (table 1 ).

TABLE 1: Loading content [wt\%] of VEGF and loading efficiency of VEGF and lysozyme (LYZ) in CSMA hydrogels, as determined indirectly by quantifying the amount of unloaded VEGF and LYZ in loading solutions by SE-UPLC. (average $\pm \mathrm{SD}, \mathrm{n}=3$ of individually prepared samples).

\begin{tabular}{|c|c|c|c|c|}
\hline \multirow{2}{*}{$\begin{array}{c}\text { loaded } \\
\text { protein(s) }\end{array}$} & \multicolumn{3}{|c|}{ VEGF } & \multirow{2}{*}{$\begin{array}{c}\text { LYZ } \\
\begin{array}{c}\text { loading efficiency } \\
\text { [\%] }\end{array}\end{array}$} \\
\hline & $\begin{array}{l}\text { feed loading content } \\
{\left[{\text { [wt } \%]^{\mathrm{a}}}^{\mathrm{a}}\right.}\end{array}$ & $\begin{array}{l}\text { measured loading } \\
\text { content }[w t \%]^{\mathrm{a}}\end{array}$ & $\begin{array}{c}\text { loading efficiency } \\
{[\%]}\end{array}$ & \\
\hline VEGF & $2.7 \pm 0.1$ & $1.8 \pm 0.2$ & $65 \pm 4$ & n.a. \\
\hline VEGF / LYZ & $2.6 \pm 0.0$ & $1.4 \pm 0.1$ & $54 \pm 3$ & $47 \pm 14$ \\
\hline VEGF + LYZ & $2.6 \pm 0.1$ & $0 \pm 0$ & $0 \pm 0$ & $36 \pm 2$ \\
\hline LYZ & n.a.* & n.a. & n.a. & $42 \pm 4$ \\
\hline
\end{tabular}

acalculated with the (estimated) dry weight of the CSMA hydrogels, expressed as weight (wt) \%, *n.a.: not applicable.

Images of the hydrogels directly after their preparation, as well as after swelling by incubation in buffer and after loading with VEGF and/or LYZ are shown in figure 4. Hydrogels before 
incubation with the protein solutions are shown as representative images, demonstrating that freshly prepared hydrogels had a diameter of $\sim 6 \mathrm{~mm}$ which increased to $\sim 10 \mathrm{~mm}$ in the low ionic strength buffer (corresponding to a 4-fold weight increase as shown in figure 5, before loading). After $24 \mathrm{~h}$ VEGF loading, the size of the hydrogels remained similar to the size before loading and they retained their initial translucent appearance, similar to hydrogels that were not loaded with protein. After loading a high amount of LYZ, however, the hydrogels became opaque and had a slightly smaller size ( $9 \mathrm{~mm}$ diameter). This appearance is attributed to the formation of complex precipitates of LYZ and CSMA, as discussed elsewhere. ${ }^{17}$
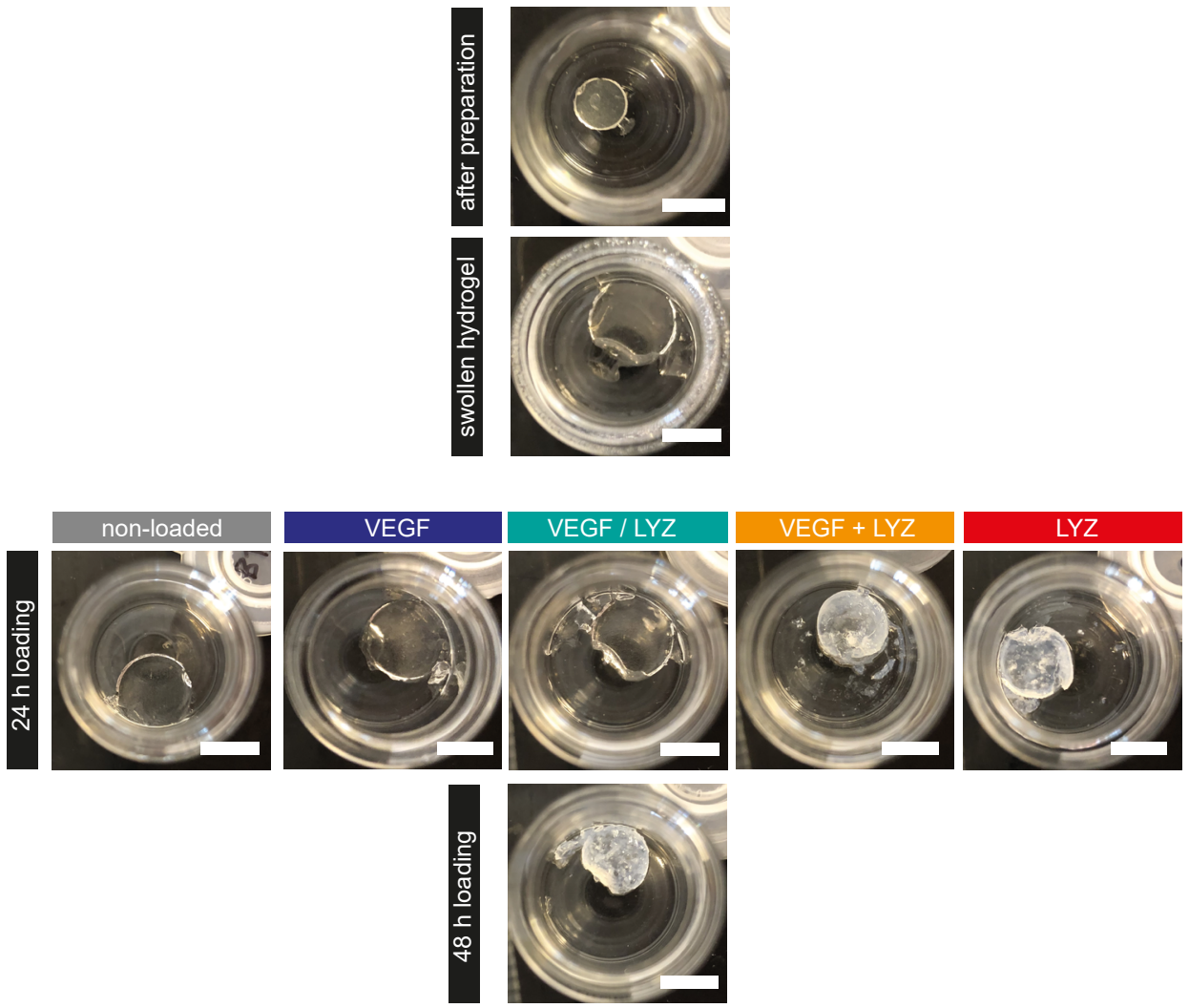

FIGURE 4: Images of the hydrogels after preparation (UV curing), after swelling in low ionic strength buffer (20 $\mathrm{mM}$ HEPES $\mathrm{pH}$ 7.4), and protein loading for 24 or $48 \mathrm{~h}$. The study groups are indicated in colored rectangles. A representative image is shown per group. White scale bar represents $7.5 \mathrm{~mm}$.

The swelling ratios of the hydrogels before and after loading of proteins are shown in figure 5 . Incubation in VEGF loading solution for 24 hours did not affect the swelling of the hydrogels (figure 5). On the other hand, incubation of the hydrogels in LYZ solution (either alone or in combination with VEGF, or sequential after the initial VEGF incubation) decreased the hydrogel swelling ratio from $\sim 4$ to $2.8-3.3$. This is caused by the formation of complex precipitates (as 
shown in figure 4), which reduces the repulsion between the negatively charged CS chains in the network due to charge neutralization upon complexation with lysozyme resulting in expulsion of water from the hydrogel network, leading to a decrease in its weight. ${ }^{17,20}$ Based on the loading efficiencies calculated for LYZ and the corresponding weight of the LYZ-CSMA complex precipitates-containing hydrogels, approximately $11 \%$ of the wet hydrogel weight can be attributed to the mass of loaded LYZ (equation S1).

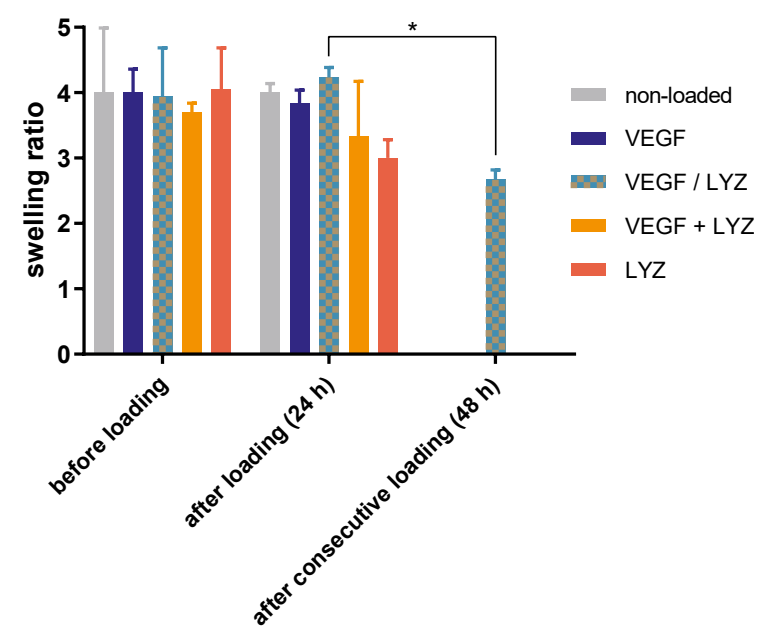

FIGURE 5: Swelling ratio (defined as the weight at the appropriate loading step divided by the weight after UVcuring) of hydrogels after loading of VEGF and/or LYZ (24 and/or 48 hours incubation. Bars represent average \pm SD ( $n=3$, individually prepared samples). *: $p<0.05$

The VEGF-loaded CSMA hydrogels with and without lysozyme co-loading were incubated at $37^{\circ} \mathrm{C}$ in a buffered saline solution with physiological ionic strength, i.e. Dulbecco's PBS $\mathrm{pH}$ 7.4. The swelling of the hydrogels in time is shown in figure 6a. For all hydrogels, a slight decrease in swelling (swelling ratio from $\sim 4$ to $\sim 3$ for VEGF-loaded hydrogels and non-loaded hydrogels, from $\sim 3$ to $\sim 2$ from LYZ-loaded hydrogels, figure 6a) was observed at the first time point of the release study. As the ionic strength of the release buffer is higher than that of the loading buffer, the negative charges on the CS chains are shielded, causing a decrease in chain-chain electrostatic repulsion. ${ }^{17,32}$ After the initial decrease, the swelling ratio of CSMA hydrogels loaded solely with VEGF and non-loaded CSMA hydrogels increased from $\sim 3$ at day 1 to $\sim 8$ after 21 days, after which the gels degraded. The hydrogels retained their transparent appearance throughout the incubation period, as shown by images of figure 7 (figure 7, nonloaded and VEGF-loaded hydrogels). 

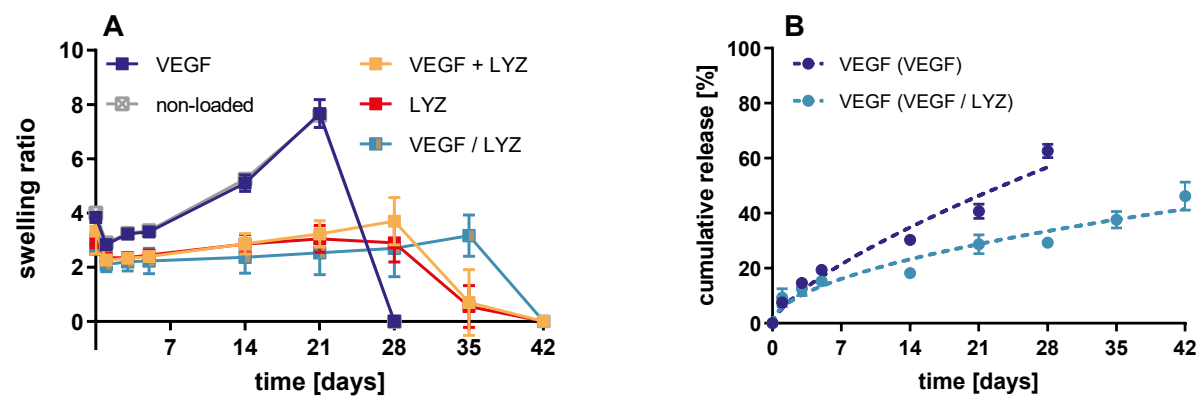

FIGURE 6: A: swelling ratio (defined as the weight of the hydrogel at the appropriate time point divided by its weight after UV-curing) of protein-loaded hydrogels during incubation in Dulbecco's PBS pH 7.4, supplemented with $0.025 \%$ Tween 20 and $0.02 \% \mathrm{NaN}_{3}$ at $37^{\circ} \mathrm{C}$. B: Cumulative release of VEGF from hydrogels. Released VEGF was quantified by SE-UPLC. Dotted lines represent Korsmeyer-Peppas model curve fit.

The swelling of hydrogels loaded with LYZ (either co-loaded with VEGF+ LYZ or solely loaded with $L Y Z$, or sequentially loaded with LYZ after VEGF loading) increased slightly from $\sim 2$ at day 1 to 3 at 28 days (VEGF + LYZ and LYZ) or 35 days (VEGF / LYZ) after which the gels degraded. Images of the hydrogels at the time point at which the hydrogels reached the highest swelling (and thus before their complete degradation) are shown in figure 7. CSMA hydrogels are biodegradable due to the ester linkage formed after reaction of a carboxylic acid group of CS with the oxirane group present in glycidylmethacrylate, as shown in figure 1.

LYZ-loaded hydrogels (VEGF / LYZ, VEGF + LYZ, LYZ) retained their opacity throughout the entire study (comparing to their appearance after LYZ loading as shown in figure 4), suggesting the continuous presence of LYZ-CSMA complex precipitates in the hydrogel network. LYZloaded hydrogels did not exhibit a distinctive swelling behavior, likely due to less chain-chain repulsion of CS chains in the network due to their charge neutralization due to complexation with lysozyme. Further, analysis of the IVR supernatants with SE-UPLC showed that 15\% of loaded LYZ was detectable as free, non-complexed LYZ (figure S4) at the end of the release study. Likely, LYZ remained complexed with the soluble CS chains formed upon hydrolysis of the hydrogel network and are therefore likely not detected by SE-UPLC.
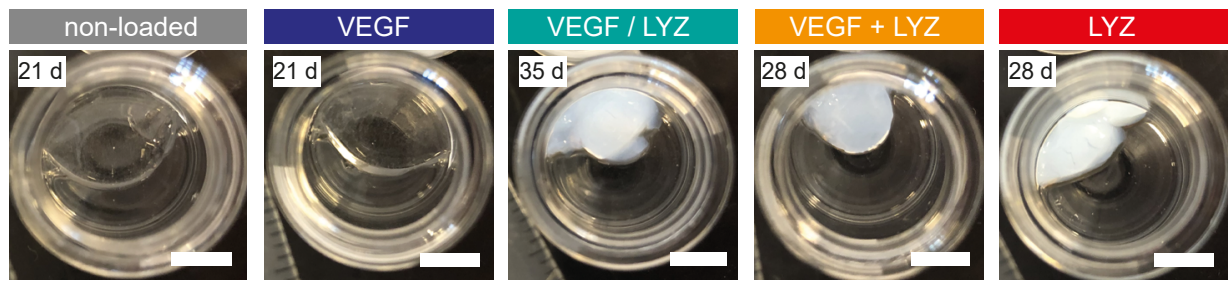

FIGURE 7: Images of protein-loaded hydrogels at the time point at which the hydrogels reached the highest swelling (incubation in Dulbecco's PBS pH 7.4, supplemented with $0.025 \%$ Tween 20 and $0.02 \% \mathrm{NaN}_{3}$ at $37{ }^{\circ} \mathrm{C}$ ). The hydrogel formulation group is indicated in the colored rectangles above each images, one representative image shown per formulation. The time point of the image is shown in the top left corner of each image (d: days). White scale bars represents $7.5 \mathrm{~mm}$. 
The release profiles of VEGF from CSMA hydrogels with and without co-loaded lysozyme are shown in figure 6b. Hydrogels loaded solely with VEGF showed continuous VEGF release up to $60 \%$ of the initially loaded amount until full degradation of the hydrogels after 28 days. The release curve was fitted using the Korsmeyer-Peppas model ${ }^{30}$ (dotted blue line in figure 6b, fit parameters shown in table 2). The calculated diffusional exponent $n(0.70)$ indicates nonFickian (anomalous) release, caused by the swelling (degradation) of the hydrogel.

The hydrogel co-loaded with lysozyme showed sustained release of VEGF for 42 days, reaching $40 \%$ of the initial loaded amount of protein. Korsmeyer-Peppas model fitting resulted in diffusional exponent of 0.53 (table 2), indicating diffusion-controlled release.

TABLE 2: Korsmeyer-Peppas model fit parameters for cumulative VEGF release curves shown in figure 6 (dotted lines).

\begin{tabular}{ccc}
\hline parameters $^{\mathbf{a}}$ & VEGF & VEGF / LYZ \\
\hline $\boldsymbol{n}$ & $0.70 \pm 0.06$ & $0.53 \pm 0.05$ \\
$\mathbf{9 5 \%} \mathbf{C l}$ & $0.58-0.85$ & $0.42-0.66$ \\
$\boldsymbol{R}^{\mathbf{2}}$ & 0.96 & 0.91 \\
\hline
\end{tabular}

${ }^{a} n$ : diffusional exponent, 95\% Cl: 95\% confidence interval, $R^{2}$ : correlation coefficient

The release of VEGF from VEGF / LYZ hydrogels was slower than the release from hydrogels loaded solely with VEGF. This indicates that LYZ-CSMA complex precipitates retard VEGF release.

As shown in figure 6a, LYZ-loaded hydrogels did not exhibit a distinctive swelling behavior, causing a lower water content (compared to hydrogels loaded solely with VEGF or nonloaded hydrogels) and thus a lower diffusivity and slower release, based on the free volume theory. ${ }^{33-35}$ Apparently, the lower diffusion coefficient (leading to slower release) dominates other effects that can lead to faster release, such as the shrinkage of gel (leading to shorter diffusion distance) and LYZ-induced weakening of the VEGF-CS interaction.

Figure $6 \mathrm{~b}$ further shows that the release of VEGF was incomplete amounting to approximately $40 \%$ total recovery of the loaded VEGF from LYZ-CSMA gels and 60\% from CSMA gels loaded with VEGF solely. Possibly, in both types of CSMA hydrogels, part of the VEGF remained complexed to soluble CS chains formed after degradation of the hydrogels which likely are not detected using the SE-UPLC analytical method.

This incomplete recovery warrants a more thorough analysis of the supernatants after complete degradation of the hydrogels. It is interesting to investigate whether VEGF is fully released in its free form and/or whether part of the VEGF is released in a complex with soluble CS. A control experiment showed that VEGF-loaded hydrogels with or without lysozyme coloading released nearly $~ 80 \%$ of free VEGF after one day incubation in a high ionic strength buffer (500 mM NaCl + 20 mM HEPES pH 7.4). After 7 days incubation, the hydrogels reached 
their highest swelling (with the exception of VEGF / LYZ hydrogel, which reached its highest swelling at day 14) and quantitative release of the loaded VEGF was measured (figure 8). This shows that the interaction of VEGF with CSMA can be disrupted by a the relatively high ionic strength of the release buffer.
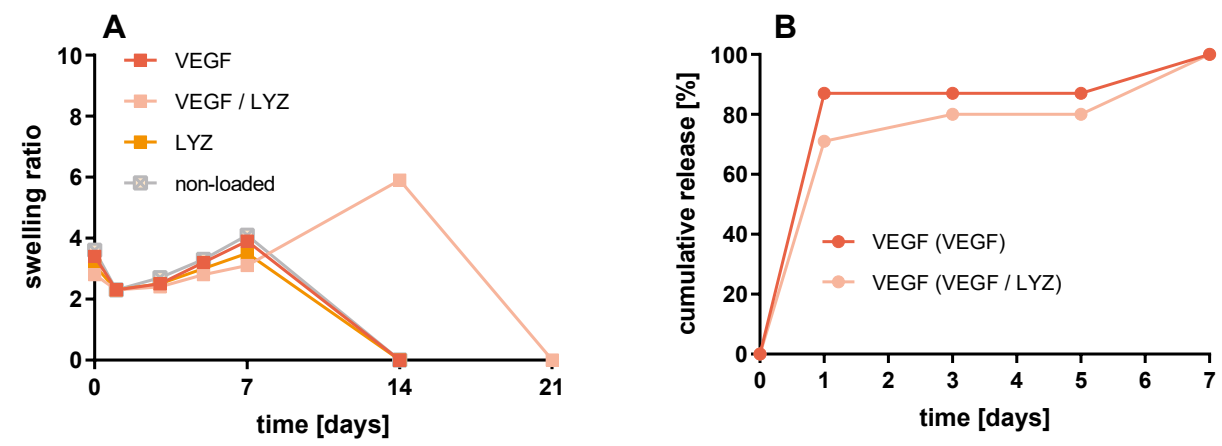

FIGURE 8: Swelling ratio of hydrogels incubated in $500 \mathrm{mM} \mathrm{NaCl}+20 \mathrm{mM} \mathrm{HEPES} \mathrm{pH} 7.4$ at $37{ }^{\circ} \mathrm{C}$ (A). B: Cumulative release of VEGF from VEGF and VEGF/LYZ hydrogels, as determined by SE-UPLC. ( $n=1$ per hydrogel formulation).

This high concentration of $\mathrm{NaCl}$ leads to shielding of the negative charge of the CS chains in the hydrogel network, leading to less swelling. However, the higher salt concentration (figure 8a) lead to a similar swelling behavior compared to incubation in phosphate buffered saline (figure 6a). Likely, the shielding of the charge of CS chains was not affected by the salt concentration above $150 \mathrm{mM}$ of $\mathrm{NaCl}$. Interestingly, the hydrogels degraded faster in buffer of $500 \mathrm{mM}$ ionic strength compared to buffer with $150 \mathrm{mM} \mathrm{NaCl}$ (figure 6a and 8a). This is rather unexpected and needs further investigation.

In future studies, we aim to investigate the applicability of the developed CSMA hydrogels and LYZ-CSMA hydrogels as a delivery system for VEGF. As stated above, several experiments are needed to further study the mechanism of VEGF release of hydrogels and to elucidate the role of LYZ-CSMA complex precipitates on VEGF release. Further, we aim to investigate the in vitro bioactivity of released VEGF with endothelial cell-based assays.

\section{Conclusions}

In this study, CSMA hydrogels were developed that exhibit sustained release of VEGF for 4 - 6 weeks. VEGF, dissolved in a low ionic strength buffer at a pH below the pl of the protein, was post-loaded in the hydrogels exploiting ionic interactions between the positively charged protein and the negatively charged CS chains of the hydrogel network. An alternative loading approach involved sequential loading of VEGF and LYZ, forming complex precipitates that retard the degradation of the hydrogel. For VEGF-loaded CSMA hydrogels, VEGF was released for 4 weeks (reaching $60 \%$ of the loaded amount), governed by swelling of the hydrogel and 
dissociation of VEGF from VEGF/CS complexes, and subsequent degradation of the hydrogels. LYZ-CSMA complex precipitate-containing CSMA hydrogels prolonged VEGF release for an additional 2 weeks (total 6 weeks). Both types of CSMA hydrogels post-loaded with VEGF demonstrated sustained release in a time frame that is suitable for vascularization of hydrogelbased tissue-engineered constructs.

\section{Acknowledgements}

This research is funded by European Union's Horizon 2020 research and innovation program under grant agreement number 645991 (Diabetes-reversing implants for enhanced viability and long-term efficacy (DRIVE)). Carl Schuurmans acknowledges a personal grant from the Future Medicines Program financed by the Netherlands Organization for Scientific Research (NWO) under grant no. 022.006.003. The authors thank Marko Mihajlovic, Sjaak Jong and Mies van Steenbergen for their excellent technical assistance. 


\section{References}

1. Langer, R.; Vacanti, J. P. Tissue engineering. Science 1993, 260, (5110), 920-6.

2. Berthiaume, F.; Maguire, T. J.; Yarmush, M. L. Tissue engineering and regenerative medicine: history, progress, and challenges. Annu Rev Chem Biomol Eng 2011, 2, 403-30.

3. Malda, J.; Visser, J.; Melchels, F. P.; Jungst, T.; Hennink, W. E.; Dhert, W. J.; Groll, J.; Hutmacher, D. W. 25th anniversary article: Engineering hydrogels for biofabrication. Adv Mater 2013, 25, (36), 5011-28.

4. Chan, B. P.; Leong, K.W. Scaffolding in tissue engineering: general approaches and tissue-specific considerations. Eur. Spine J. 2008, 17 Suppl 4, 467-79.

5. O'Brien, F. J. Biomaterials \& scaffolds for tissue engineering. Materials Today 2011, 14, (3), 88-95.

6. Thomas, D.; O'Brien, T.; Pandit, A. Toward customized extracellular niche engineering: progress in cellentrapment technologies. Adv Mater 2018, 30, (1), 1703948.

7. Rouwkema, J.; Rivron, N. C.; van Blitterswijk, C. A. Vascularization in tissue engineering. Trends Biotechnol. 2008, 26, (8), 434-41.

8. Buwalda, S. J.; Boere, K. W.; Dijkstra, P. J.; Feijen, J.; Vermonden, T.; Hennink, W. E. Hydrogels in a historical perspective: from simple networks to smart materials. J. Control. Release 2014, 190, 254-73.

9. Wang, H.; Heilshorn, S. C. Adaptable hydrogel networks with reversible linkages for tissue engineering. Adv Mater 2015, 27, (25), 3717-36.

10. Yang, J.; Zhang, Y. S.; Yue, K.; Khademhosseini, A. Cell-laden hydrogels for osteochondral and cartilage tissue engineering. Acta Biomater. 2017, 57, 1-25.

11. Freudenberg, U.; Liang, Y.; Kiick, K. L.; Werner, C. Glycosaminoglycan-based biohybrid hydrogels: a sweet and smart choice for multifunctional biomaterials. Adv Mater 2016, 28, (40), 8861-8891.

12. Prestwich, G. D. Hyaluronic acid-based clinical biomaterials derived for cell and molecule delivery in regenerative medicine. J. Control. Release 2011, 155, (2), 193-9.

13. Gandhi, N. S.; Mancera, R. L. The structure of glycosaminoglycans and their interactions with proteins. Chem. Biol. Drug Des. 2008, 72, (6), 455-82.

14. Abbadessa, A.; Mouser, V. H. M.; Blokzijl, M. M.; Gawlitta, D.; Dhert, W. J. A.; Hennink, W. E.; Malda, J.; Vermonden, T. A synthetic thermosensitive hydrogel for cartilage bioprinting and its biofunctionalization with polysaccharides. Biomacromolecules 2016, 17, (6), 2137-2147.

15. Khanlari, A.; Suekama,T.C.; Detamore,M.S.; Gehrke, S.H. Structurally diverse and readily tunable photocrosslinked chondroitin sulfate based copolymers. Journal of Polymer Science Part B: Polymer Physics 2015, 53, (15), 10701079.

16. Lim, D. K.; Wylie, R. G.; Langer, R.; Kohane, D. S. Selective binding of C-6 OH sulfated hyaluronic acid to the angiogenic isoform of VEGF(165). Biomaterials 2016, 77, 130-138.

17. Schuurmans, C. C. L.; Abbadessa, A.; Bengtson, M. A.; Pletikapic, G.; Eral, H. B.; Koenderink, G.; Masereeuw, R.; Hennink, W. E.; Vermonden, T. Complex coacervation-based loading and tunable release of a cationic protein from monodisperse glycosaminoglycan microgels. Soft Matter 2018, 14, (30), 6327-6341.

18. Lim, J. J.; Hammoudi, T. M.; Bratt-Leal, A. M.; Hamilton, S. K.; Kepple, K. L.; Bloodworth, N. C.; McDevitt, T. C.; Temenoff, J.S. Development of nano- and microscale chondroitin sulfate particles for controlled growth factor delivery. Acta Biomater. 2011, 7, (3), 986-95.

19. Wetter, L. R.; Deutsch, H. F. Immunological studies on egg white proteins. IV. Immunochemical and physical studies of lysozyme. J. Biol. Chem. 1951, 192, (1), 237-42.

20. Comert, F.; Malanowski, A. J.; Azarikia, F.; Dubin, P. L. Coacervation and precipitation in polysaccharide-protein systems. Soft Matter 2016, 12, (18), 4154-61.

21. Park, M. R.; Chun, C.; Cho, C. S.; Song, S. C. Enhancement of sustained and controlled protein release using polyelectrolyte complex-loaded injectable and thermosensitive hydrogel. Eur. J. Pharm. Biopharm. 2010, 76, (2), 179-88.

22. Yla-Herttuala, S.; Rissanen, T. T.; Vajanto, I.; Hartikainen, J. Vascular endothelial growth factors: biology and current status of clinical applications in cardiovascular medicine. J. Am. Coll. Cardiol. 2007, 49, (10), 1015-26. 


\section{Chapter 5}

23. Ferrara, N.; Gerber, H. P.; LeCouter, J. The biology of VEGF and its receptors. Nat. Med. 2003, 9, (6), 669-76.

24. Smink, A. M.; Li, S.; Hertsig, D. T.; de Haan, B. J.; Schwab, L.; van Apeldoorn, A. A.; de Koning, E.; Faas, M. M.; Lakey, J. R.; de Vos, P. The efficacy of a prevascularized, retrievable poly(D,L,-lactide-co-epsilon-caprolactone) subcutaneous scaffold as transplantation site for pancreatic islets. Transplantation 2017, 101, (4), e112-e119.

25. Silva, E. A.; Mooney, D. J. Effects of VEGF temporal and spatial presentation on angiogenesis. Biomaterials 2010, $31,(6), 1235-41$.

26. Davies, N.; Dobner, S.; Bezuidenhout, D.; Schmidt, C.; Beck, M.; Zisch, A. H.; Zilla, P. The dosage dependence of VEGF stimulation on scaffold neovascularisation. Biomaterials 2008, 29, (26), 3531-8.

27. Amsden, B. G.; Timbart, L.; Marecak, D.; Chapanian, R.; Tse, M. Y.; Pang, S. C. VEGF-induced angiogenesis following localized delivery via injectable, low viscosity poly(trimethylene carbonate). J. Control. Release 2010, 145, (2), 109-15.

28. Ferrara, N.; Leung, D. W.; Cachianes, G.; Winer, J.; Henzel, W. J. Purification and cloning of vascular endothelial growth factor secreted by pituitary folliculostellate cells. Methods Enzymol. 1991, 198, 391-405.

29. Abbadessa, A.; Blokzijl, M. M.; Mouser, V. H. M.; Marica, P.; Malda, J.; Hennink, W. E.; Vermonden, T. A thermoresponsive and photo-polymerizable chondroitin sulfate-based hydrogel for 3D printing applications. Carbohydrate Polymers 2016, 149, 163-174.

30. Ritger, P. L.; Peppas, N. A. A simple equation for description of solute release II. Fickian and anomalous release from swellable devices. J. Control. Release 1987, 5, 37 - 42.

31. Shah, R. B.; Schwendeman, S. P. A biomimetic approach to active self-microencapsulation of proteins in PLGA. J. Control. Release 2014, 196, 60-70.

32. Rydzewski, R. Swelling and shrinking of a polyelectrolyte gel induced by a salt solution. Continuum Mechanics and Thermodynamics 1990, 2, (2), 77-97.

33. Feil, H.; Bae, Y. H.; Feijen, J.; Kim, S. W. Molecular separation by thermosensitive hydrogel membranes. J. Membr. Sci. 1991, 64, (3), 283-294.

34. van Dijk-Wolthuis, W. N. E.; Hoogeboom, J. A. M.; van Steenbergen, M. J.;Tsang, S. K. Y.; Hennink, W. E. Degradation and release behavior of dextran-based hydrogels. Macromolecules 1997, 30, (16), 4639-4645.

35. Sato, S.; Kim, S. Macromolecular diffusion through polymer membranes. Int. J. Pharm. 1984, 22, (2-3), $229-255$. 


\section{Supporting Information}

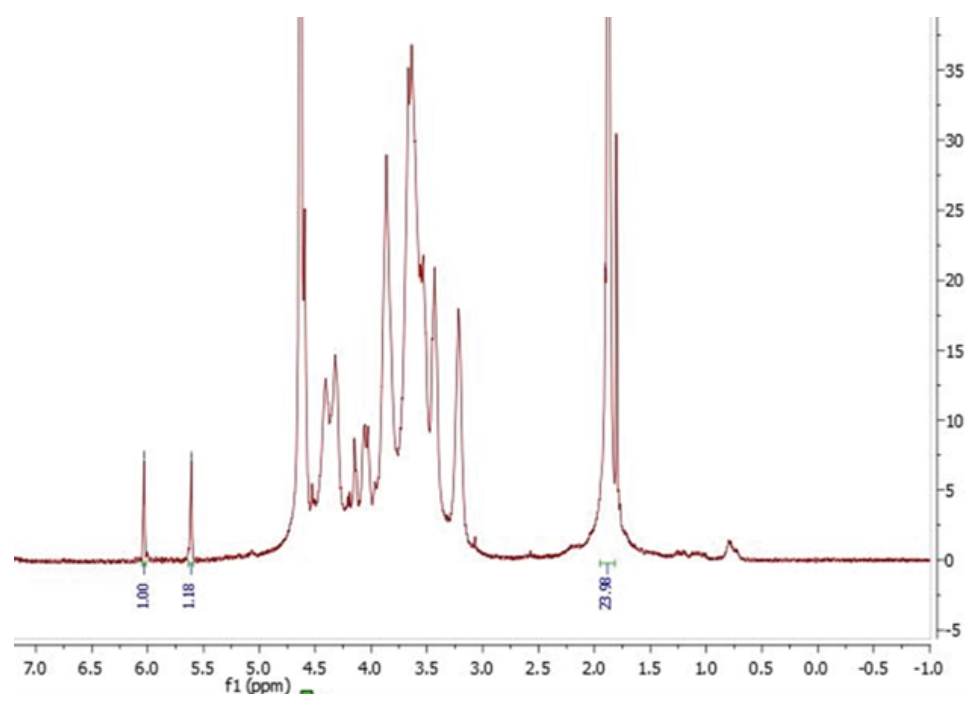

FIGURE S1: ${ }^{1} \mathrm{H}-\mathrm{NMR}$-spectrum of CSMA for calculating the degree of methacrylation of chondroitin sulfate (equation 1).

A

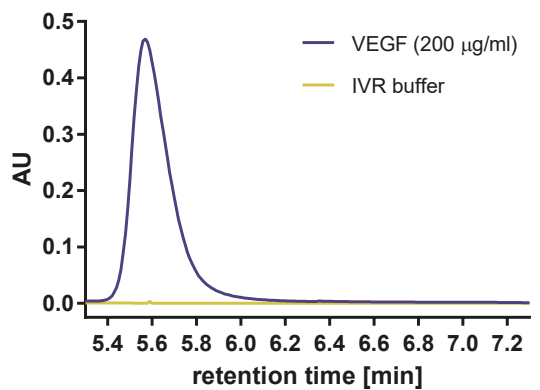

B

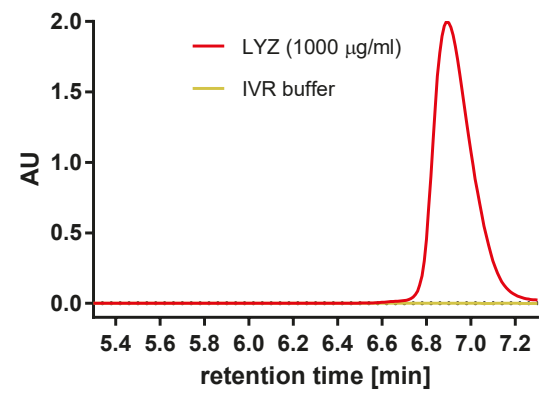

FIGURE S2: SE-UPLC chromatograms of VEGF and LYZ in IVR buffer. A: VEGF (200 $\mu \mathrm{g} / \mathrm{ml})$ had a retention times of $\sim 5.5$ minutes. B: LYZ $(1000 \mu \mathrm{g} / \mathrm{ml})$ had a retention time of $\sim 6.8$ minutes. 

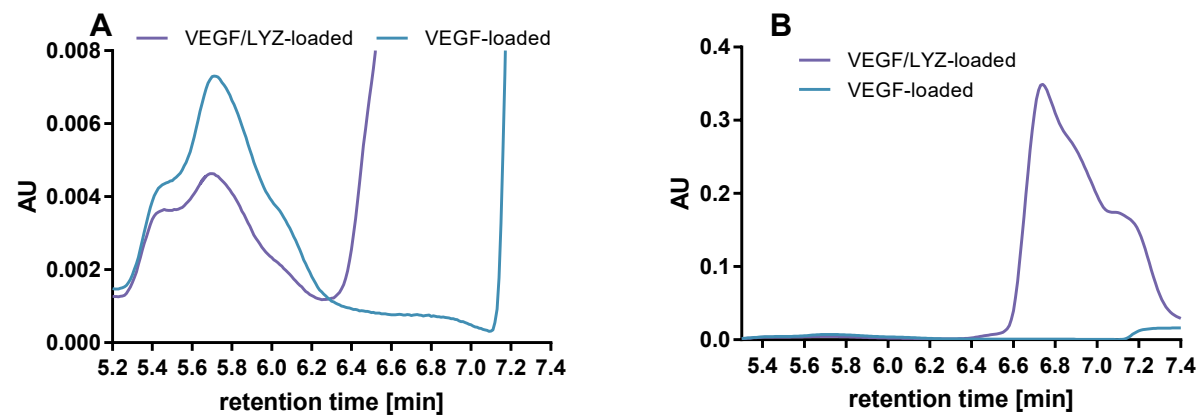

FIGURE S3: SE-UPLC chromatograms of IVR samples of day 1. A: Peaks of released VEGF from VEGF-loaded hydrogels and VEGF/LYZ-loaded hydrogels at a retention time of $~ 5.4$ minutes. B: Peak of released LYZ from VEGF/LYZ-loaded hydrogels at a retention time of $\sim 6.6$ minutes.

A

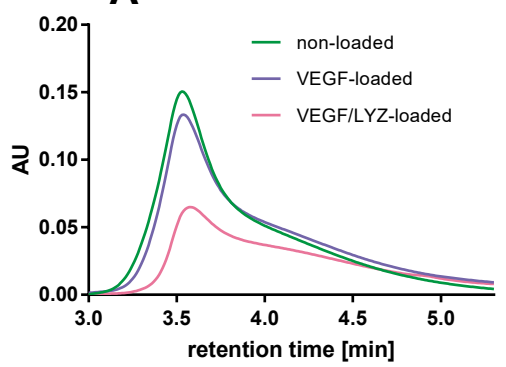

B

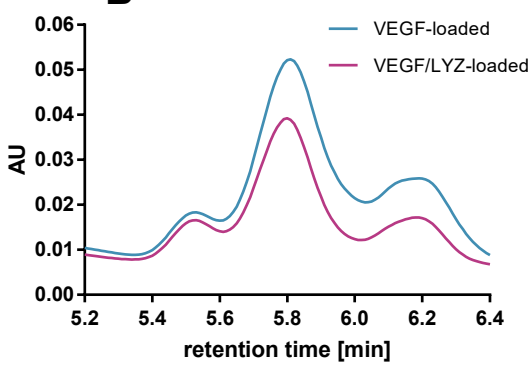

C

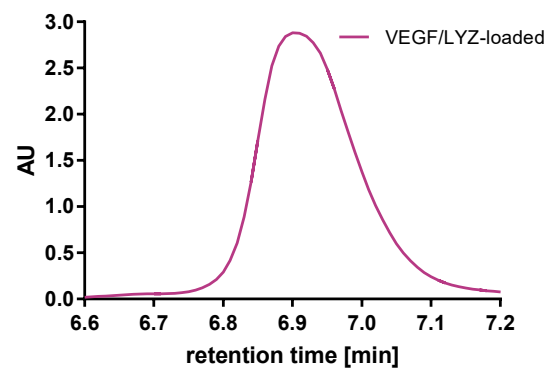

FIGURE S4: SE-UPLC chromatograms of IVR samples of day 21. A: Peaks of CSMA degradation products at a retention time of 3.5 minutes from VEGF-loaded, VEGF/LYZ-loaded and non-loaded hydrogels. B: Peaks of released VEGF from VEGF-loaded hydrogels and VEGF/LYZ-loaded hydrogels at a retention time of $\sim 5.5$ minutes. C: Peak of released LYZ from VEGF/LYZ-loaded hydrogels at a retention time of $\sim 6.8$ minutes.

LYZ in hydrogel $(\%)=\frac{\text { weight loaded LYZ }(\sim 20 \mathrm{mg})}{\text { weight }(\text { wet }) \text { hydrogel after LYZ loading }(\sim 185 \mathrm{mg})}=0.11 \times 100=11 \%$

EQUATION S1: Amount of LYZ in hydrogels [\%] 


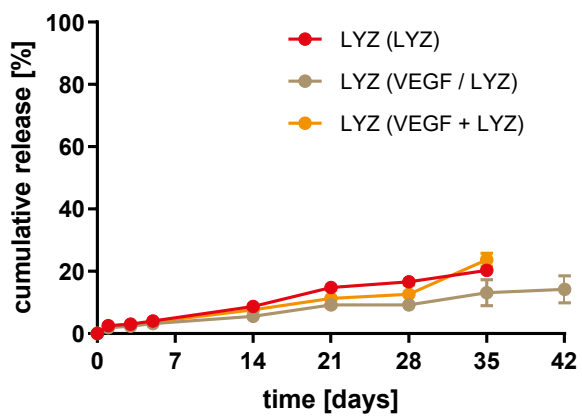

FIGURE S5: Cumulative release of soluble, non-complexed lysozyme (LYZ) from hydrogels incubated in Dulbecco's PBS pH 7.4, 0.025\% Tween 20,0.02\% $\mathrm{NaN}_{3}$ at $37^{\circ} \mathrm{C}$. LYZ was quantified by SE-UPLC. Average \pm SD $(n=3)$ 



\section{Chapter 6}

Summary and Perspectives 


\section{Summary}

The work described in this thesis was part the European consortium DRIVE (Diabetes-reversing implants with enhanced viability and long-term efficacy), which was funded by European Union's Horizon 2020 research and innovation program. The aim of the DRIVE consortium was to develop a pancreatic islet transplantation device that functions as an "artificial pancreas" for treating diabetes type 1.

One of the major hurdles towards clinical success of such a pancreatic islet transplantation device is the poor islet survival due to insufficient supply of nutrients and oxygen. To tackle this, microspheres that release vascular endothelial growth factor (VEGF) in an appropriate time frame for the induction of blood vessel formation (angiogenesis) are included in the devices that are developed for the DRIVE approach for encapsulation of pancreatic islets. As a result, the device will be vascularized, thereby supplying islets with oxygen and nutrients and removing waste products via the blood circulation.

The work in this thesis focused on the development of sustained release formulations of VEGF and other proangiogenic growth factors via mild, protein-friendly encapsulation methods. In this context, endothelial cell based assays were applied for evaluating the bioactivity of released growth factors. Special emphasis was laid on obtaining a target release time frame of four weeks, which has been shown as ideal for stable blood vessel formation in rodent models. $^{1-3}$

Chapter 1 of this thesis provides a general introduction on diabetes mellitus type 1 and pancreatic islet transplantation as a novel, promising treatment approach. Encapsulation of pancreatic islets in biomaterial devices can be classified as an advanced therapy medicinal product (ATMP) aiming for increased transplantation efficacy by providing an immunedeprived, organ-like environment. As mentioned above, such an organ-like environment is relying on the formation of a vascular network that surrounds the device. Proangiogenic factors that are released from depot formulations into the surrounding tissue can stimulate that formation of such a vascular network. Chapter 1 discusses polymeric microspheres and hydrogels as attractive sustained release systems for such a local delivery approach.

In Chapter 2, we reported the development and characterization of VEGF-loaded microspheres based on blends of two [poly( $\varepsilon$-caprolactone)-poly(ethylene glycol)-poly( $\varepsilon$ caprolactone)]-[poly(L-lactic acid)] ([PCL-PEG-PCL]-b-[PLLA]) multiblock copolymers. Monodisperse microspheres $(40-50 \mu \mathrm{m})$ with a narrow size distribution were prepared by a solvent evaporation-based membrane emulsification method and showed a loading of $\sim 0.79$ wt\% and high loading efficiency ( 78\%). Microspheres prepared with an optimized blend ratio of the two polymers (i.e. prepared with a 50:50 blend) released VEGF continuously for four weeks at $37^{\circ} \mathrm{C}$ in a diffusion-controlled manner as determined by ELISA and SE-UPLC, whereby the PEG molecular weight and PEG content of the polymers essentially affected 
the release kinetics. This release profile was preserved after repeated and long-term storage of the microspheres at $-20^{\circ} \mathrm{C}$, demonstrating excellent storage stability of the formulation. Importantly, released VEGF was bioactive throughout the entire release study period.

In Chapter 3, the microspheres described in Chapter $\mathbf{2}$ were incorporated in a 3D-printed poly(dimethylsiloxane) (PDMS) device intended for future rodent studies in which the DRIVE devices are filled with pancreatic islets. The porous morphology of silicone-based devices was elucidated by scanning electron microscopy (SEM) and their high permeability was demonstrated by release studies with fluorescent or colored model compounds. A dispersion of VEGF-loaded microspheres in hyaluronic acid carrier liquid was injected into the device via a custom-made inlet. VEGF was released continuously from the device for four weeks at $37^{\circ} \mathrm{C}$ in an in vitro setting as determined by ELISA, whereby the device did not form a barrier for release of VEGF from the device. The bioactivity of released VEGF was demonstrated by an endothelial cell-based proliferation assay. Taken together, such microsphere-incorporated silicone devices present an attractive prevascularization strategy for inducing vascularization towards the implanted device prior to filling of pancreatic islets.

In Chapter 4, a post-loading method for proangiogenic growth factors in microspheres, termed active self-encapsulation (ASE), was investigated for simultaneous loading and release of a combination of VEGF, fibroblast growth factor (FGF) and insulin-like growth factor (IGF). ASE is a two-step process, where a positively charged protein (growth factor) is "captured" by ionic interaction with negatively charged polysaccharides (trapping agents) that have been formulated in porous PLGA microspheres. Subsequently, pore closure of the microspheres is induced at elevated temperature above the glass transition temperature of PLGA, thereby entrapping the growth factor. In this study, we compared high molecular weight dextran sulfate (HDS), chondroitin sulfate (CS) and hyaluronic acid (HA) as trapping agents for encapsulating VEGF. Porous PLGA microspheres (67 - $81 \mu \mathrm{m}$ ) were prepared by a double emulsion solvent evaporation method, whereby porosity was induced through the sugar-leaching agent trehalose. While no VEGF was loaded in HA-containing microspheres, VEGF loading efficiency reached 100\% (4.5 wt\% loading) and 28\% (1.3 wt\% loading) for microspheres that contained the trapping agents HDS or CS, respectively. This results suggest that sulfate groups present in dextran sulfate- and chondroitin sulfate are essential for the interaction of VEGF with the trapping agents. Bioactive VEGF was released continuously from HDS-containing microspheres for four weeks at $37^{\circ} \mathrm{C}$, as determined by ELISA. CS-containing microspheres showed a high burst release, followed by continuous release of bioactive VEGF up to four weeks. A 1:1:1 combination (weight ratio) of VEGF, FGF and IGF was co-loaded in PLGA microspheres containing HDS as trapping agent, resulting in a high loading (4.3 wt\%) and loading efficiency (91\%). The release of VEGF and FGF from these microspheres was sustained for four weeks, while IGF was nearly entirely released at the first time point of the release study ( 1 week). Further, the bioactivity of released growth factors throughout the entire release study was preserved as demonstrated by an endothelial cell proliferation 
assay and VEGF bioreceptor assay. A combination of VEGF, FGF and IGF resulted in a stronger angiogenic response compared to each single growth factor, indicating a potentiating angiogenic effect of FGF and VEGF.

In Chapter 5, we described the loading of VEGF in methacrylated chondroitin sulfate (CSMA) hydrogels for achieving sustained release. VEGF was loaded in CSMA hydrogels through ionic interaction with negatively charged sulfate groups of CSMA in low ionic strength buffer. We investigated the co-loading of VEGF with a high concentration of lysozyme (LYZ) which is known to form complex precipitates with CSMA. Such LYZ-CSMA complex precipitates will change the swelling of the hydrogel and may prolong the release window of VEGF. VEGF was loaded in CSMA hydrogels with high loading efficiencies, i.e. 65\% when loaded solely, and $54 \%$ when VEGF loading was followed by LYZ loading. For hydrogels loaded solely with VEGF, release at $37^{\circ} \mathrm{C}$ was sustained for 4 weeks up to the degradation and collapse of the hydrogel reaching $60 \%$ cumulative release, as measured by SE-UPLC. Release was governed by a combination of diffusion and bulk degradation of the hydrogels. VEGF release from LYZCSMA complex precipitate-containing hydrogels was sustained for 6 weeks until hydrogel collapse and amounted to a total release of 40\%. This latter type of hydrogels retained their opaque appearance and hardly increased in w/w ratio until complete degradation, suggesting a delaying effect of LYZ-CSMA complex precipitates on VEGF release. These chondroitin sulfate-based hydrogels, exhibiting sustained VEGF release, are an attractive tissue-engineered scaffold with potential use as a prevascularization strategy.

\section{Perspectives}

In this thesis, three sustained release formulations are described for VEGF and a combination of VEGF, FGF and IGF which exhibited continuous bioactive growth factor release for a period of four weeks. We have shown that the microspheres developed in Chapter $\mathbf{2}$ can be incorporated in a silicone device for future use as a prevascularization strategy (Chapter 3), which should be feasible for microspheres developed in Chapter $\mathbf{4}$ as well. Similarly, the system presented in Chapter $\mathbf{5}$ is likewise attractive for use as a prevascularization strategy, given the biodegradability of the hydrogel and sustained growth factor release. Nevertheless, further investigation and optimization are necessary towards clinical translation of these systems.

\section{Considerations for the quantification of released growth factors}

Proteins, such as the growth factors described in this thesis, have complex structures and specific folding that are essential for their therapeutic effect in vivo. In this thesis, growth factors released from microspheres and hydrogels were quantified by ELISA (Chapter 2,

Chapter $\mathbf{3}$ and Chapter $\mathbf{4}$ ) and SE-UPLC (Chapter $\mathbf{2}$ and Chapter 5). For quantification by ELISA, the recovery rates of VEGF release at the end of the release studies were low, ranging 
from $10-40 \%$. Although the recovery rates for released VEGF were higher by SE-UPLC (40 $60 \%)$, incomplete release was also observed. A direct comparison of ELISA and SE-UPLC for quantifying released VEGF in Chapter 2 revealed a systematic underestimation of the VEGF content by ELISA. As discussed in Chapter 2, this could be due to loss of protein through adsorption to pipet tips and plastic test tubes during the numerous dilution steps towards the working range of the assay. As ELISA is based on the binding of (labelled) antibodies to a specific epitope of VEGF, possibly the formulation process altered the tertiary structure of the protein in such a way that the epitope was not detectable anymore, for example through the exposure to shear stresses or organic solvents. Compared to ELISA, SE-UPLC is less sensitive, as no dilution steps are necessary and the protein is separated based on its size and detected by UV absorbance. Apart from an analytical reason, irreversible immobilization of the protein in the polymeric system could also be an explanation for incomplete release leading to low recovery. Nevertheless, the bioactivity of released growth factors was demonstrated, showing that ELISA-non-detectable growth factorNEGF is bioactive. This indicates that the low release observed with ELISA is rather of analytical origin than a sign of protein degradation or instability. Low recovery rates of cumulative VEGF release quantified by ELISA have been reported in previous studies. ${ }^{4-6}$ In line with the findings presented in this thesis, bioactivity of released growth factor was demonstrated in vitro and in vivo.4-6 Taken together, great care must be taken in selecting and developing an analytical method for release studies. Further, the determination of the bioactivity of released growth factor is essential for evaluating a sustained release formulation.

\section{Bioactivity determination of released growth factors}

The work in this thesis shows that growth factors VEGF and FGF released from microspheres were bioactive, as determined by an endothelial cell proliferation assay. However, proliferation of endothelial cells is merely one of many processes involved in angiogenesis in vivo. ${ }^{7}$ Other in vitro assays have been developed to study angiogenesis processes such as endothelial cell tube formation, migration and differentiation.7. 8 A combination of in vitro assays can give more insights into the bioactivity of released growth factors and prediction of in vivo efficacy?

\section{Considerations for obtaining functional angiogenesis in vivo}

The formation of stable blood vessels, i.e. functional angiogenesis, towards a pancreatic islet transplantation device is essential for its clinical success. In vivo, angiogenesis is an interplay of many growth factors and cytokines. ${ }^{9,} 10$ A combination of growth factors for induction of angiogenesis has been shown as advantageous ${ }^{11-13}$, in line with the findings in Chapter $\mathbf{4}$ of this thesis. In this regard, the system described in Chapter 4, where a combination of growth factors can be loaded and released simultaneously, is advantageous. Loading a combination 
of growth factors in microspheres developed in Chapter $\mathbf{2}$ is also feasible, as well as for the hydrogels reported in Chapter 5. However, as the microspheres described in Chapter $\mathbf{4}$ and hydrogels developed in Chapter $\mathbf{5}$ rely on ionic interaction of a positively charged growth factor with a negatively charged polysaccharide, a high isoelectric point of the growth factor intended for loading is required.

\section{Considerations for the production of microspheres}

Challenges also remain in the translational aspect of microspheres. The large-scale production of microspheres is generally associated with high costs, and therefore efficient production ways are warranted. ${ }^{14}$ Membrane emulsification, as described in Chapter $\mathbf{2}$, is an efficient method for preparing uniform particles with high loading efficiencies. ${ }^{15,16}$ An important advantage of the microspheres developed in Chapter $\mathbf{4}$ is that they can be sterilized post production, thereby reducing costs and unnecessary exposure of the protein to sterilization methods. ${ }^{17}$

\section{Clinical translation of the DRIVE pancreatic islet transplantation device}

The pancreatic islet transplantation device developed by the DRIVE consortium stands out due to its multicomponent set up. The current strategy involves suspending pancreatic islets in hyaluronic acid carrier liquid with oxygen precursors and subsequent filling of the device and subcutaneous implantation. Ultimately, the biocompatibility of pancreatic islets in this setup is essential.

For combining this setup with growth factor-loaded microspheres, two approaches are feasible. Firstly, prevascularization of the device is possible through filling of VEGF-releasing microspheres in devices and implantation prior to filling with islets, as discussed in Chapter $\mathbf{3}$. Similarly, the microspheres developed in Chapter $\mathbf{4}$ could also be incorporated in this device according to the protocol reported in Chapter $\mathbf{3}$.

A second possibility is to disperse the microspheres in the initial islet suspension. In that case, the viability of pancreatic islets with microspheres, and released VEGF, needs to be ensured. Further consideration should be drawn to the fact that the components of the suspension could alter and possibly delay VEGF release from the device, and lead to insufficient VEGF levels in the surrounding tissue. 


\section{Conclusions}

Pancreatic islet transplantation is a novel treatment approach for diabetes type 1 which can increase patients' quality of life tremendously. However, the efficacy of this treatment is still low due to poor islet survival, amongst others. Recent developments have focused on encapsulating pancreatic islets in biomaterial devices, which offer an immune-isolated, organ-like environment. Clinical success relies on long-term islet survival through the functional integration of the device in vivo, i.e. vascularization of the device. Proangiogenic growth factors, incorporated into the device and continuously present for a period of four weeks, can stimulate stable blood vessel growth towards the device. In this thesis, sustained release formulations based on microspheres and hydrogels were developed which continuously released bioactive growth factors over a period of four weeks. Therefore, incorporation of these sustained release formulations in diabetes-reversing implants is a promising strategy for promoting their vascularization, bringing pancreatic islet transplantation one step closer to clinical reality. 


\section{References}

1. Silva, E. A.; Mooney, D. J. Effects of VEGF temporal and spatial presentation on angiogenesis. Biomaterials 2010, 31, (6), 1235-41.

2. Amsden, B. G.; Timbart, L.; Marecak, D.; Chapanian, R.; Tse, M. Y.; Pang, S. C. VEGF-induced angiogenesis following localized delivery via injectable, low viscosity poly(trimethylene carbonate). J. Control. Release 2010, 145, (2), 109-15.

3. Davies, N.; Dobner, S.; Bezuidenhout, D.; Schmidt, C.; Beck, M.; Zisch, A. H.; Zilla, P. The dosage dependence of VEGF stimulation on scaffold neovascularisation. Biomaterials 2008, 29, (26), 3531-8.

4. Quinlan, E.; Lopez-Noriega, A.; Thompson, E. M.; Hibbitts, A.; Cryan, S. A.; O'Brien, F. J. Controlled release of vascular endothelial growth factor from spray-dried alginate microparticles in collagen-hydroxyapatite scaffolds for promoting vascularization and bone repair. J. Tissue Eng. Regen. Med. 2017, 11, (4), 1097-1109.

5. O’Dwyer, J.; Murphy, R.; Dolan, E. B.; Kovarova, L.; Pravda, M.; Velebny, V.; Heise, A.; Duffy, G. P.; Cryan, S. A. Development of a nanomedicine-loaded hydrogel for sustained delivery of an angiogenic growth factor to the ischaemic myocardium. Drug Deliv Trans/ Res 2019.

6. des Rieux, A.; Ucakar, B.; Mupendwa, B. P.; Colau, D.; Feron, O.; Carmeliet, P.; Preat, V. 3D systems delivering VEGF to promote angiogenesis for tissue engineering. J. Control. Release 2011, 150, (3), 272-8.

7. Staton, C. A.; Reed, M. W.; Brown, N. J. A critical analysis of current in vitro and in vivo angiogenesis assays. Int. J. Exp. Pathol. 2009, 90, (3), 195-221.

8. Auerbach, R.; Lewis, R.; Shinners, B.; Kubai, L.; Akhtar, N. Angiogenesis assays: a critical overview. Clin. Chem. 2003, 49, (1), 32-40.

9. Said, S. S.; Pickering, J. G.; Mequanint, K. Advances in growth factor delivery for therapeutic angiogenesis. J. Vasc. Res. 2013, 50, (1), 35-51.

10. Novosel, E. C.; Kleinhans, C.; Kluger, P. J. Vascularization is the key challenge in tissue engineering. Adv Drug Deliv Rev 2011, 63, (4-5), 300-11.

11. Kim, S. K.; Lee, J.; Song, M.; Kim, M.; Hwang, S. J.; Jang, H.; Park, Y. Combination of three angiogenic growth factors has synergistic effects on sprouting of endothelial cell/mesenchymal stem cell-based spheroids in a 3D matrix. J. Biomed. Mater. Res. B Appl. Biomater. 2016, 104, (8), 1535-1543.

12. Liu, G.; Pareta, R. A.; Wu, R.; Shi, Y.; Zhou, X.; Liu, H.; Deng, C.; Sun, X.; Atala, A.; Opara, E. C.; Zhang, Y. Skeletal myogenic differentiation of urine-derived stem cells and angiogenesis using microbeads loaded with growth factors. Biomaterials 2013, 34, (4), 1311-26.

13. Cittadini, A.; Monti, M. G.; Petrillo, V.; Esposito, G.; Imparato, G.; Luciani, A.; Urciuolo, F.; Bobbio, E.; Natale, C. F.; Sacca, L.; Netti, P. A. Complementary therapeutic effects of dual delivery of insulin-like growth factor-1 and vascular endothelial growth factor by gelatin microspheres in experimental heart failure. Eur. J. Heart Fail. 2011, 13, (12), 1264-74.

14. Brown, L. R. Commercial challenges of protein drug delivery. Expert Opin Drug Deliv 2005, 2, (1), $29-42$.

15. Ma, G. Microencapsulation of protein drugs for drug delivery: strategy, preparation, and applications. J. Control. Release 2014, 193, 324-40.

16. Piacentini, E.; Dragosavac, M.; Giorno, L. Pharmaceutical Particles Design by Membrane Emulsification: Preparation Methods and Applications in Drug Delivery. Curr. Pharm. Des. 2017, 23, (2), 302-318.

17. Desai, K. G.; Kadous, S.; Schwendeman, S. P. Gamma irradiation of active self-healing PLGA microspheres for efficient aqueous encapsulation of vaccine antigens. Pharm. Res. 2013, 30, (7), 1768-78. 


\section{Appendices}

Nederlandse Samenvatting

Zusammenfassung in deutscher Sprache

Curriculum Vitae

List of Publications

Acknowledgements 



\section{Nederlandse Samenvatting}

Het werk dat in dit proefschrift wordt beschreven was onderdeel van het Europese consortium DRIVE (Diabetes-reversing implants with improved viability and enhanced efficacy), dat werd gefinancierd door het Horizon 2020 onderzoeks- en innovatieprogramma van de Europese Unie. De doelstelling van het DRIVE-consortium was om een "kunstmatige alvleesklier" te ontwikkelen die bestaat uit een biomateriaal waarin insuline-producerende cellen normaal functioneren. Zo'n kunstmatige alvleesklier kan in de toekomst mogelijk gebruikt worden voor patiënten die zelf geen insuline kunnen maken (patiënten met suikerziekte, diabetes mellitus type 1). Insuline is een essentieel stofwisselingshormoon voor het reguleren van het glucose gehalte in het bloed. Patiënten met diabetes mellitus type 1 moeten hun leven lang insuline injecteren, maar bij patiënten met ernstige diabetes is dit niet voldoende. Een transplantatie van de eilandjes van Langerhans (het onderdeel van de alvleesklier dat de insuline producerende cellen bevat) biedt mogelijk een uitkomst voor deze patiënten. Zulke transplantaties worden nu ook al uitgevoerd, en de kunstmatige alvleesklier van het DRIVE project moet de slagingskans van zo'n transplantatie verhogen.

Eén van de grootste hindernissen voor het klinische succes van een transplantatie van eilandjes van Langerhans is de slechte overleving van de insuline producerende cellen als gevolg van de immunologische afweer van het lichaam tegen het transplantaat. Het DRIVE consortium wil deze problemen oplossen door de eilandjes in een kunstmatig orgaan in te kapselen dat dit tegengaat. Een ander groot probleem is de toevoer van voedingsstoffen en zuurstof naar het getransplanteerde weefsel. Het werk in dit proefschrift was gericht op het verbeteren van de toevoer van zuurstof en voedingsstoffen naar de cellen in de kunstmatige alvleesklier. Om dit aan te pakken, zijn microsferen die vascular endothelial growth factor (VEGF) vrijgeven in het biomateriaal ingebouwd. VEGF is een groeifactor die de bloedvatvorming (angiogenese) stimuleert. Als gevolg hiervan zal het kunstmatig orgaan worden gevasculariseerd, waardoor de eilandjes van Langerhans zuurstof en voedingsstoffen kunnen krijgen en afvalproducten en insuline aan de bloedcirculatie worden afgegeven. In dit proefschrift is met name onderzoek gedaan naar de ontwikkeling van ontwikkeling van formuleringen via milde, eiwitvriendelijke methoden.

Hoofdstuk 1 van dit proefschrift geeft een algemene inleiding over diabetes mellitus type 1 en transplantatie van eilandjes van Langerhans als een nieuwe, veelbelovende behandelingsmethode. Inkapseling van eilandjes in biomaterialen kan worden geclassificeerd als een geneesmiddel voor geavanceerde therapie (advanced therapy medicinal product (ATMP)) dat gericht is op verhoogde transplantatie-effectiviteit door een orgaanachtige omgeving te bieden die beschermt tegen de immunologische afweer van de patiënt. Zoals hierboven vermeld, moet een biomateriaal dat een dergelijke orgaanachtige omgeving nabootst omgeven worden met een vasculair netwerk dat het kunstmatige orgaan met de bloedsomloop verbindt. Proangiogene groeifactoren die vrijkomen uit depotformuleringen 
in het omringende weefsel kunnen die vorming van zo'n vasculair netwerk stimuleren. Hoofdstuk 1 bespreekt polymere microsferen en hydrogelen als aantrekkelijke systemen voor een dergelijke lokale afgifte van groeifactoren.

In hoofdstuk 2 wordt de ontwikkeling en karakterisering van met VEGF beladen polymere microsferen beschreven die gemaakt zijn met mengsels van [poly( $\varepsilon$-caprolacton)poly(ethyleenglycol)-poly( $\varepsilon$-caprolacton)][(L-melkzuur)] multiblok copolymeren ([PCL-PEGPCL]-b-[PLLA]). Polymere microsferen met een nauwe grootteverdeling (40 - $50 \mu \mathrm{m}$ ) werden bereid middels een membraan-emulsificatieproces en vertoonden een belading van $\sim 0,8$ w/w\% en hoge beladingsefficiëntie ( 78\%). Microsferen met een 1:1 mengverhouding van de twee polymeren gaven VEGF met het gewenste profiel af, dat wil zeggen met een continue afgifte gedurende vier weken. In proefdierstudies is aangetoond dat een vierweekse afgifte van VEGF voldoende is voor stabiele bloedvatvorming rondom een biomateriaal. De afgifte van VEGF werd bepaald door de microsferen te incuberen in een geschikte buffer bij $37^{\circ} \mathrm{C}$ waarna het afgegeven VEGF werd gemeten met ELISA en SE-UPLC. Belangrijk is dat vrijgegeven VEGF bioactief was gedurende de gehele studieperiode van afgifte; dit werd onderzocht door te testen of het afgegeven VEGF effect heeft op de groeisnelheid van endotheelcellen. Het afgifteprofiel van de polymere microsferen werd behouden na herhaalde en langdurige opslag van de microsferen bij - $20^{\circ} \mathrm{C}$, wat belangrijke informatie is voor de andere partners in het DRIVE consortium.

In hoofdstuk 3 werden de microsferen (beschreven in hoofdstuk 2) opgenomen in een 3D-geprint poly (dimethylsiloxaan) (PDMS) kunstmatige alvleesklier die bedoeld is voor proefdierexperimenten van het DRIVE consortium. De poreuze morfologie van op siliconen gebaseerde biomaterialen werd onderzocht door scanning-elektronenmicroscopie (SEM) en hun hoge permeabiliteit werd aangetoond met experimenten waarin het transport van fluorescente of gekleurde modelverbindingen werd onderzocht. Vervolgens werd een dispersie van met VEGF beladen microsferen in een hyaluronzuur-dragervloeistof werd in het biomateriaal geïnjecteerd. De afgifte van VEGF vanuit het biomateriaal werd bestudeerd bij $37^{\circ} \mathrm{C}$ in een in vitro setting vergelijkbaar met die in hoofdstuk 2. De experimenten hebben aangetoond dat het PDMS materiaal geen extra barrière vormde voor afgifte van VEGF, en dat er een 4-weekse afgifte bereikt werd van bioactief VEGF. Het inbouwen van VEGF-beladen microsferen in het PDMS biomateriaal is een aantrekkelijke strategie voor prevascularisatie van de kunstmatige alvleesklier, waarbij het biomateriaal eerst geïmplanteerd wordt en er een netwerk van bloedvaten gevormd wordt voordat de eilandjes van Langerhans in het biomateriaal ingespoten worden. In hoofdstuk 4 werd een alternatieve methode beschreven voor het formuleren van VEGF in polymere microsferen. Deze zogeheten post-load methode, actieve zelf-insluiting (active self-encapsulation (ASE)) genoemd, is een tweestapsproces, waarbij eerst een negatief geladen polysaccharide (vangmiddel; trapping agent) wordt geformuleerd in poreuze PLGA-microsferen. Deze negatief geladen polysacchariden kunnen vervolgens positief 
geladen eiwitten (groeifactoren) "invangen" door ionische interactie, waarna de poriën van de microsferen gesloten kunnen worden door de microsferen te incuberen bij een temperatuur boven de glasovergangstemperatuur van PLGA. Het uiteindelijke resultaat is dat de groeifactoren ingesloten kunnen worden in de microsferen bij relatief milde condities, dat wil zeggen zonder de groeifactoren bloot te stellen aan organische oplosmiddelen of emulsificatieprocessen. De ASE beladingsmethode werd onderzocht voor de belading (en afgifte) van microsferen met de proangiogene groeifactoren VEGF, fibroblast growth factor (FGF) en insulin-like growth factor (IGF). Bovendien werd onderzocht of het mogelijk is om een formulering te ontwikkelen voor de gelijktijdige belading en afgifte van deze groeifactoren uit polymere microsferen. In deze studie werden eerst de polysacchariden dextransulfaat (HDS; dextraansulfaat polymeren meteen hoog molecuulgewicht), chondroïtinesulfaat (CS) en hyaluronzuur (HA) vergeleken als vangmiddelen voor het inkapselen van VEGF. Poreuze PLGA-microbolletjes (67 - $81 \mu \mathrm{m}$ ) werden bereid door een dubbele emulsie methode waarbij de hulpstof trehalose ingebouwd werd om poreuze microsferen te verkrijgen. De microsferen met HDS hadden de hoogste ASE belading nadat ze met VEGF geïncubeerd werden (100\% beladingsefficiëntie bij een $4.5 \mathrm{w} / \mathrm{w} \%$ belading). Microsferen met CS hadden een onvolledige belading (28\% efficiëntie) terwij microsferen met HA geen ASE belading vertoonden. Deze resultaten suggereren dat sulfaatgroepen aanwezig in dextraansulfaat en chondroïtinesulfaat essentieel zijn voor de interactie van VEGF met de vangmiddelen (HA heeft geen sulfaat groepen en HDS heeft de hoogste fractie sulfaatgroepen). Experimenten waarbij de VEGFbeladen microsferen gedurende vier weken bij $37^{\circ} \mathrm{C}$ werden geïncubeerd in een buffer toonden het gewenste continue afgifteprofiel uit HDS-bevattende microsferen. CS-bevattende microsferen vertoonden een hoge burst-afgifte, gevolgd door continue afgifte van VEGF tot vier weken. Een 1:1:1 combinatie (gewichtsverhouding) van VEGF, FGF en IGF werd ook efficiënt geladen in PLGA-microsferen die HDS als vangmiddel bevatten, overeenkomend met een laadefficiëntie van 91\%. De afgifte van VEGF en FGF uit deze microsferen bleef vier weken aanhouden, terwijl IGF voornamelijk werd vrijgegeven gedurende het begin van de afgifte-studie (week 1 van de incubatie). De bioactiviteit van de geformuleerde groeifactoren werd gedurende het gehele afgifteprofiel - behouden zoals aangetoond middels een endotheliale celproliferatietest en VEGF-receptoractivatie in een bioreceptor assay. Een combinatie van VEGF, FGF en IGF resulteerde in de sterkste proangiogene respons. Hieruit kon geconcludeerd worden dat dit combinatiepreparaat voor de simultane afgifte van proangiogene groeifactoren veelbelovend is, aangezien er een versterkend angiogeen effect van VEGF, FGF en mogelijk IGF kan optreden.

In hoofdstuk 5 werd een derde afgiftesysteem voor de formulering van VEGF onderzocht. Ditmaal werden methacrylaat chondroïtinesulfaat (CSMA) hydrogelen met VEGF beladen. CSMA-hydrogelen bevaten negatief geladen sulfaatgroepen die positief geladen VEGF kunnen binden door middel van ionische interactie. Er werd onderzocht wat het effect is van het gelijktijdig laden van VEGF met een hoge concentratie van een ander positief geladen 
eiwit, lysozyme (LYZ). Eerder onderzoek heeft aangetoond dat een hoge concentratie LYZ complexe precipitaten vormt met CSMA. Zulke complexe precipitaten zullen de zwelling van de hydrogel veranderen en kunnen het afgifteprofiel van VEGF verlengen. VEGF werd geladen in CSMA-hydrogelen met hoge beladingsefficiëntie, d.w.z. 65\% wanneer de hydrogelen alleen met VEGF werden geladen, en 54\% wanneer VEGF belading werd gevolgd door een incubatiestap met een hoge concentratie LYZ. Voor hydrogelen die uitsluitend metVEGF waren beladen, werd een 4-weeks afgifteprofiel bij $37^{\circ} \mathrm{C}$ gevonden, terwijl de VEGF-LYZ hydrogelen een 6-weeks afgifteprofiel hadden. De afgifte werd bepaald door een combinatie van diffusie en bulkafbraak van de hydrogelen. Deze op chondroïtinesulfaat gebaseerde hydrogelen zijn minder geschikt voor het inbouwen in een kunstmatige alvleesklier maar kunnen mogelijk gebruikt wel gebruikt worden voor de lokale afgifte van groeifactoren in de omgeving van het kunstmatige orgaan. Daarmee zijn ze geschikt voor en prevascularisatiestrategie waarbij het bloedvatnetwerk rondom het kunstmatige orgaan wordt aangelegd voordat de eilandjes van Langerhans in het biomateriaal worden getransplanteerd.

Hoofdstuk 6 vat de uitkomsten van dit proefschrift samen. De transplantatie van eilandjes van Langerhans in een biomateriaal als "kunstmatige alvleesklier" is een veelbelovende behandelmethode voor diabetes type 1 patiënten. Voor klinisch succes is langdurige overleving van getransplanteerde eilandjes essentieel. Dit kan worden bereikt door functionele integratie van het orgaan in vivo, d.w.z. door vascularisatie van het kunstmatige orgaan. Proangiogene groeifactoren, ingebouwd in het kunstmatige orgaan, en welke gedurende een periode van vier weken aanwezig zijn, kunnen stabiele bloedvatgroei naar het orgaan stimuleren. In dit proefschrift werden de ontwikkeling van formuleringen met vertraagde afgifte, zoals microsferen en hydrogels, van proangiogene groeifactoren beschreven. Deze formuleringen zijn veelbelovend voor vascularisatie van diabetes-terugdraaiende implantaten. Hierdoor kan de slagingskans van een kunstmatige pancreas worden verhoogd en deze nieuwe behandelmethode dichter bij de patiënt komen. 


\section{Zusammenfassung in deutscher Sprache}

Das Forschungsprojekt dieser Dissertation war Teil des europäischen Konsortiums DRIVE (Diabetes-reversing implants with improved viability and enhanced efficacy), das vom Forschungsund Innovationsprogramm Horizon 2020 der Europäischen Union finanziert wurde. Ziel des DRIVE-Konsortiums war es, eine künstliche Bauchspeicheldrüse zu entwickeln, die aus einem Biomaterial besteht, in dem insulinproduzierende Zellen ihre biologische Funktion erfüllen können. Eine solche künstliche Bauchspeicheldrüse kann in Zukunft für Patienten verwendet werden, die selbst kein Insulin herstellen können (Patienten mit Diabetes, Typ1-Diabetes mellitus). Insulin ist ein essentielles Stoffwechselhormon zur Regulierung des Blutzuckerspiegels. Patienten mit Typ-1-Diabetes mellitus müssen ein Leben lang Insulin injizieren, was jedoch bei Patienten mit schwerem Diabetes nicht ausreicht. EineTransplantation der Langerhans'schen Inseln (der Teil der Bauchspeicheldrüse, der die insulinproduzierenden Zellen enthält) könnte eine Lösung für diese Patienten darstellen. Solche Transplantationen werden bereits jetzt durchgeführt, und die künstliche Bauchspeicheldrüse des DRIVEKonsortiums dürfte die Erfolgschancen einer solchen Transplantation erhöhen.

Eine der größten Hürden für den klinischen Erfolg einer Langerhans'schen Inseltransplantation ist die schlechte Überlebensrate der insulinproduzierenden Zellen aufgrund der körpereigenen Immunabwehr gegen das Implantat. Das DRIVE-Konsortium will dieses Problem lösen indem es die Langerhans'schen Inseln in ein künstliches Organ einbaut und dadurch vor dem Immunsystem schützt. Ein weiteres Problem ist die Versorgung des transplantierten Gewebes mit Nährstoffen und Sauerstoff. Ziel dieser Dissertation war es, die Versorgung der Langerhans'schen Inseln in der künstlichen Bauchspeicheldrüse mit Sauerstoff und Nährstoffen zu gewährleisten. Um dies zu ermöglichen, werden Mikrosphären in das Biomaterial eingebaut, die den vascular endothelial growth factor (VEGF) freisetzen. VEGF ist ein Wachstumsfaktor, der die Blutgefäßbildung (Angiogenese) stimuliert. Infolgedessen wird das künstliche Organ vaskularisiert, so dass einerseits die Langerhans'schen Inseln Sauerstoff und Nährstoffe erhalten und andererseits Abfallprodukte und im Wesentlichen Insulin in den Blutkreislauf gelangen können. In dieser Dissertation wurde insbesondere die Entwicklung von Formulierungen einer künstlichen Bauchspeicheldrüse mit milden, proteinfreundlichen Methoden erforscht.

Kapitel 1 dieser Arbeit bietet eine allgemeine Einführung in Typ-1-Diabetes mellitus und die Transplantation von Langerhans'schen Inseln als neue, vielversprechende Behandlungsmethode. Die Einkapselung von Langerhans'schen Inseln in Biomaterialien kann als Arzneimittel für neuartige Therapien (advanced therapy medicinal product, ATMP) eingestuft werden, mit dem die Wirksamkeit der Transplantation gesteigert werden soll, indem eine biologisch-organähnliche Umgebung bereitgestellt wird. Wie oben erwähnt, muss ein Biomaterial, das eine solche organähnliche Umgebung nachahmt, von einem Gefäßnetz umgeben sein, das das künstliche Organ mit dem Blutkreislauf verbindet. Proangiogene 
Wachstumsfaktoren, die aus den Biomaterialen des künstlichen Organs verzögert freigesetzt werden, können die Bildung eines solchen Gefäßnetzwerks stimulieren. In Kapitel 1 werden polymere Mikrosphären und Hydrogele als attraktive Systeme für eine solche lokale Abgabe von Wachstumsfaktoren erörtert.

In Kapitel 2 wird die Entwicklung und Charakterisierung von VEGF-beladenen polymerischen Mikrosphären beschrieben die aus Gemischen von [Poly( $\varepsilon$-Caprolacton) -Poly(ethylenglykol) -Poly(ع-Caprolacton)]-[(L-Milchsäure)] - Multiblock-kopolymeren ([PCL-PEG-PCL]-b-[PLLA]) hergestellt wurden. Mikrosphären mit einer engen Größenverteilung (40 - $50 \mu \mathrm{m}$ ) wurden durch ein Membranemulgierungsverfahren hergestellt und zeigten eine Beladung von $\sim$ 0,8 w/w\% und eine hohe Beladungseffizienz ( 78\%). Mikrosphären mit einem 1:1-Mischungsverhältnis der beiden Polymere setzten VEGF mit dem gewünschten Profil frei, d.h. mit einer kontinuierlichen Freisetzung für vier Wochen. Verschiedene veröffentlichte Tierstudien haben gezeigt, dass eine vierwöchige Freisetzung von VEGF für eine stabile Blutgefäßbildung um ein Biomaterial herum ausreicht. Die Freisetzung von VEGF wurde durch Inkubieren der Mikrosphären in einem geeigneten Puffer bei $37^{\circ} \mathrm{C}$ bestimmt, wonach freigesetztes VEGF durch ELISA und SE-UPLC quantifiziert wurde. Wichtig zu erwähnen ist, dass freigesetztes VEGF während des gesamten Freisetzungszeitraums bioaktiv war. Dies wurde untersucht, indem getestet wurde, ob freigesetztes VEGF einen Einfluss auf die Wachstumsrate von Endothelzellen hat. Das Freisetzungsprofil der Mikrosphären blieb nach mehrmaliger und längerer Lagerung bei $-20^{\circ} \mathrm{C}$ erhalten, was eine wichtige Information für die weiterführenden Projekte anderer Partner des DRIVE-Konsortiums darstellt.

In Kapitel 3 wurden die Mikrosphären (beschrieben in Kapitel 2) in eine 3D-gedruckte künstliche Bauchspeicheldrüse aus Poly(dimethylsiloxan) (PDMS) eingeschlossen, die für Tierversuche des DRIVE-Konsortiums vorgesehen war. Die poröse Morphologie dieser silikonbasierten Biomaterialien wurde durch Rasterelektronenmikroskopie (REM) untersucht. Die hohe Permeabilität des PDMS-Biomaterials wurde durch Experimente bezüglich des Transportes fluoreszierender oder gefärbter Modellverbindungen nachgewiesen. Eine Dispersion von VEGF-beladenen Mikrosphären in einer Hyaluronsäureträgerflüssigkeit wurde danach in das Biomaterial injiziert. Die Freisetzung von VEGF aus dem Biomaterial wurde bei $37{ }^{\circ} \mathrm{C}$ in einer ähnlichen in-vitro-Umgebung wie in Kapitel 2 untersucht. Die Experimente haben gezeigt, dass das PDMS-Material der künstlichen Bauchspeicheldrüse keine zusätzliche Barriere für die Freisetzung von VEGF bildete und dass nach wie vor eine Freisetzung von bioaktivem VEGF über den Zeitraum von vier Wochen erreicht wurde. Der Einbau von VEGFbeladenen Mikrosphären in das PDMS-Biomaterial ist eine vielversprechende Strategie für eine Prävaskularisation der künstlichen Bauchspeicheldrüse, in dem das Biomaterial zuerst implantiert und sich ein VEGF-induziertes Blutgefäßnetzwerk bilden kann, bevor die Langerhans'schen Inseln in das Biomaterial injiziert werden. 
In Kapitel 4 wurde eine alternative Methode zur Formulierung von VEGF in polymerischen Mikrosphären beschrieben. Diese sogenannte post-loading-Methode, genannt Active SelfEncapsulation (ASE), ist ein zweistufiger Prozess, bei dem zunächst ein negativ geladenes Polysaccharid (Einfangmittel; trapping agent) in porösen PLGA-Mikrosphären formuliert wird. Diese negativ geladenen Polysaccharide können daraufhin positiv geladene Proteine (Wachstumsfaktoren) durch ionische Wechselwirkung "einfangen“, wonach die Poren der Mikrosphären geschlossen werden indem die Mikrosphären bei einer Temperatur oberhalb der Glasübergangstemperatur von PLGA inkubiert werden. Das Endergebnis ist, dass die Wachstumsfaktoren unter relativ milden Bedingungen in den Mikrosphären eingeschlossen werden können, d.h. Wachstumsfaktoren werden weder organischen Lösungsmitteln noch Emulgierungsprozessen ausgesetzt. Die ASE-Beladungsmethode wurde zur Beladung (und Freisetzung) von Mikrosphären mit den proangiogenen Wachstumsfaktoren VEGF, fibroblast growth factor (FGF) und insulin-like growth factor (IGF) untersucht. Darüber hinaus wurde untersucht, ob es möglich ist diese drei Wachstumsfaktoren gleichzeitig in Mikrosphären zu beladen und dadurch gleichzeitig freizusetzen. Für dieses Experiment wurden zunächst die Polysaccharide Dextransulfat (HDS; high molecular weight dextran sulfate), Chondroitinsulfat (CS) und Hyaluronsäure (HA) als Einfangmittel für die Beladung von VEGF verglichen. Poröse PLGA-Mikrosphären (67 bis $81 \mu \mathrm{m}$ ) wurden durch ein Doppelemulsionsverfahren hergestellt, bei dem Trehalose als Hilfsmittel eingearbeitet wurde, um die Porosität zu gewährleisten. Die Mikrosphären mit HDS hatten die höchste ASE-Beladung nach Inkubation mit VEGF (100\% Beladungseffizienz bei einer Beladung von 4,5 w/w\%). Mikrosphären mit CS hatten eine unvollständige Beladung (28\% Beladungseffizienz), während Mikrosphären mit HA keine ASE-Beladung zeigten. Diese Ergebnisse legten nahe, dass die in HDS und CS vorhandenen Sulfatgruppen für die Wechselwirkung mit VEGF notwendig sind (HA enthält keine Sulfatgruppen; HDS hat den höchsten Sulfatgruppengehalt). HDS-haltige Mikrosphären setzten VEGF im gewünschten verzögerten Freisetzungsprofil frei, d.h. innerhalb von vier Wochen. CS-haltige Mikrosphären zeigten zunächst eine hohe burst-Freisetzung, gefolgt von einer kontinuierlichen Freisetzung von VEGF für bis zu vier Wochen. Eine 1:1:1-Kombination (Gewichtsverhältnis) von VEGF, FGF und IGF wurde ebenfalls effizient in PLGA-Mikrosphären geladen die HDS als Einfangmittel enthielten, d.h. mit einer Beladungseffizienz von 91\%. Die Freisetzung von VEGF und FGF aus diesen Mikrosphären dauerte vier Wochen, während IGF hauptsächlich zu Beginn der Freisetzungsstudie (Woche 1 der Inkubation) freigesetzt wurde. Die Bioaktivität der formulierten Wachstumsfaktoren wurde während des gesamten Freisetzungsprofils aufrechterhalten, wie durch einen Endothelzellproliferationstest und eine VEGF-Rezeptoraktivierung in einem Biorezeptortest gezeigt wurde. Eine Kombination von VEGF, FGF und IGF führte zu der stärksten proangiogenen Reaktion. Daraus konnte konkludiert werden, dass dieses Kombinationspräparat zur gleichzeitigen Freisetzung von proangiogenen Wachstumsfaktoren vielversprechend ist, da eine verstärkende angiogene Wirkung von VEGF, FGF und möglicherweise IGF auftreten kann. 
In Kapitel 5 wurde ein drittes System für die Freisetzung von VEGF untersucht. Hier wurden Methacrylatchondroitinsulfat (CSMA) -Hydrogele anstelle von Mikrosphären mit VEGF beladen. CSMA-Hydrogele enthalten negativ geladene Sulfatgruppen, die durch ionische Wechselwirkung positiv geladenes VEGF binden können. Der Effekt der gleichzeitigen Beladung von VEGF mit einer hohen Konzentration eines anderen positiv geladenen Proteins, Lysozym (LYZ), wurde untersucht. Bereits veröffentlichte Studien haben gezeigt, dass eine hohe Konzentration von LYZ mit CSMA complex precipitates (komplexe Niederschläge) bildet. Complex precipitates beeinflussen die Quellung des Hydrogels und können das Freisetzungsprofil von VEGF verlängern. VEGF wurde mit einer hohen Beladungseffizienz in CSMA-Hydrogele geladen, nämlich 65\% nach Inkubation ausschließlich mit VEGF und 54\% nach einer darauffolgenden Inkubation mit einer hohen Konzentration LYZ. Für Hydrogele die ausschließlich mit VEGF beladen waren, wurde ein vierwöchiges Freisetzungsprofil bei $37{ }^{\circ} \mathrm{C}$ gemessen, während die VEGF-LYZ-Hydrogele ein sechswöchiges Freisetzungsprofil zeigten. Die Freisetzung wurde durch eine Kombination von Diffusion und bulk-Erosion der Hydrogele bestimmt. Diese auf Chondroitinsulfat basierenden Hydrogele sind für den Einbau in einer künstlichen Bauchspeicheldrüse weniger geeignet, können jedoch möglicherweise für die lokale Abgabe von Wachstumsfaktoren in die Umgebung des künstlichen Organs verwendet werden. Dies macht sie für eine Prävaskularisierungsstrategie geeignet, bei der das Blutgefäßnetzwerk um das künstliche Organ gelegt wird, bevor die Langerhans'schen Inseln in das Biomaterial transplantiert werden.

In Kapitel 6 werden die Ergebnisse dieser Dissertation zusammengefasst und diskutiert. Die Transplantation von Langerhans'schen Inseln in einem Biomaterial als "künstliche Bauchspeicheldrüse" ist eine vielversprechende Behandlungsmethode für Typ-1Diabetespatienten. Das langfristige Überleben transplantierter Zellen ist für den klinischen Erfolg von entscheidender Bedeutung. Dies kann durch die funktionelle Integration des Organs in vivo, d.h. durch Vaskularisation des künstlichen Organs erreicht werden. Proangiogene Wachstumsfaktoren die in das künstliche Organ eingebaut sind und über einen Zeitraum von vier Wochen freigesetzt werden, können das stabile Wachstum der Blutgefäße rundum das künstliche Organ stimulieren. Diese Dissertation beschreibt die Entwicklung von Formulierungen (Mikrosphären und Hydrogele) mit verzögerter Freisetzung von proangiogenen Wachstumsfaktoren. Diese Formulierungen sind vielversprechend für die Vaskularisation einer künstlichen Bauchspeicheldrüse. Hierdurch kann die Erfolgsrate einer Transplantation von Langerhans'schen Inseln erhöht werden, und diese neuartige Behandlungsmethode kann dadurch einen Schritt näher zur klinischen Anwendung gebracht werden. 


\section{Curriculum Vitae}

Karina Scheiner (1988) holds a PharmD (2014) and MSc in Drug Research (2013) from the University of Bonn, Germany. For her master thesis project, she studied the metabolic engineering of bacteria through co-localization of enzymes with small affinity proteins (affibodies) at the School of Biotechnology of the KTH Royal Institute of Technology in Stockholm, Sweden. From 2014 to 2015, she worked within pharmaceutical product development in the Netherlands. In July 2015, she started her PhD project at the Department of Pharmaceutics at Utrecht University, the Netherlands, under the supervision of Prof. dr. ir. Wim Hennink and Dr. Robbert Jan Kok. This project focused on the development of sustained release formulations of growth factors, as part of the Horizon 2020 consortium DRIVE, which aimed at developing a bioartificial pancreas for diabetes mellitus type 1 treatment. Her PhD project included collaborations with several universities and SMEs across Europe, among which InnoCore BV (Groningen, the Netherlands), University College Dublin (Dublin, Ireland), Abiel (Palermo, Italy), Explora Biotech (Rome, Italy) and Boston Scientific (Galway, Ireland). In 2018, Karina was a visiting research scholar at the Department of Pharmaceutical Sciences at the University of Michigan (Ann Arbor, USA) under the supervision of Dr. Steven Schwendeman. This research stay was funded by a travel grant from the Foundation "De Drie Lichten". The results of the PhD project are described in this thesis. 



\section{List of Publications}

\section{This thesis}

K.C. Scheiner, R.F. Maas-Bakker, M.J. van Steenbergen, S.P. Schwendeman, W.E. Hennink, R.J. Kok, Self-encapsulation of Proangiogenic Growth Factors in PLGA Microspheres. Manuscript in preparation.

K.C. Scheiner, F. Coulter, R.F. Maas-Bakker, G. Ghersi, T.T. Nguyen, R. Steendam, G.P. Duffy, W.E. Hennink, E.D. O'Cearbhaill, R. J. Kok, Vascular Endothelial Growth Factor-Releasing Microspheres Based on Poly(epsilon-Caprolactone-PEG-epsilon-Caprolactone)-b-Poly(L-Lactide) Multiblock Copolymers Incorporated in a Three-Dimensional Printed Poly(Dimethylsiloxane) Cell Macroencapsulation Device. J. Pharm. Sci. 2020, 109, (1), 863-870

K. C. Scheiner, R.F. Maas-Bakker, T.T. Nguyen, A.M. Duarte, G. Hendriks, L. Sequeira, G.P. Duffy, R. Steendam, W.E. Hennink, R.J. Kok, Sustained release of vascular endothelial grwoth factor from poly(epsilon-caprolactone-PEG-epsilon-caprolactone)-b-poly(l-lactide) multiblock copolymer microspheres. ACS Omega 2019, 4, (7), 11481-11492

\section{Other publications}

J.A.W. Jong, Y. Guo, D. Hazenbrink, S. Douka, D. Verdijk, J. van der Zwan, K. Houben, M. Baldus, K.C. Scheiner, R. Dalebout, M.C. Verhaar, R. Smakman, W.E. Hennink, K.G.F. Gerritsen, C.F. van Nostrum, A Ninhydrin-type Urea Sorbent for the Development of a Wearable Artificial Kidney. Macromol. Biosci. 2020, in press

J.A.W. Jong, Y. Guo, C. Veenhoven, M.E. Moret, J. van der Zwan, A. Lucini Paioni, M. Baldus, K.C. Scheiner, R. Dalebout, M.J. van Steenbergen, M.C. Verhaar, R. Smakman, W.E. Hennink, K.G.F. Gerritsen, C.F. van Nostrum, Phenylglyoxaldehyde-functionalized Polymeric Sorbents for Urea Removal from Aqueous Solutions. ACS Appl. Polym. Mater. 2019, in press 



\section{Acknowledgements}

Better than reading a thousand books is travelling a thousand miles.

Better than travelling a thousand miles is getting to know a thousand people.

Chinese Proverb

Many people have contributed to this thesis, and in fact it is the people that I have met during the last four years that have made my PhD journey particularly memorable. I am truly grateful for the help and support of many people, which I would like to express on the following pages.

First of all, I want to thank my promotor, Wim. Thank you for your supervision and support. I appreciate that you took the time every month to sit with me to discuss my progress. Although you have been very critical at times, it improved the quality of my work and I learned a lot from your feedback. Your dedication, hard work and immense knowledge is truly impressive. I would also like to thank you for your quick review of my manuscripts and swift responses to my emails or other requests.

Robbert Jan, my copromotor, thank you for your supervision, guidance and support. No matter how busy you were, I could always come by your office with any matter, question or concern. I especially appreciate your quick review of my manuscripts (sometimes even within one day (!)), deliverable reports and other requests. I admire your pragmatism, especially in times when I could not see the wood for the trees. Most of all, I want to thank you for the great time we had during all the trips to consortium meetings across Europe.

Thank you, Roel, for your involvement in the cell culture experiments during my PhD. Thank you for your hard work, dedication and support. Thank you for planning every cell culture experiments very carefully. I could always rely on you, and I admire your scientific integrity.

One of the perks of being a PhD candidate within a Horizon 2020 consortium is attending many meetings across Europe. At all these meetings I met many talented scientists and professionals from research fields different than mine, such as stem cell biology, medical device development and many others. I also got to collaborate with many universities and companies, which I enjoyed very much. I want to thank everybody from the DRIVE consortium, for their expertise, advice, support and cooperation. After each meeting, I returned to Utrecht with a boost of motivation. There are a few people that I want to thank specifically. Giulio and Monica, thank you for hosting Fergal and me for the in vivo studies in Palermo. Eduardo, thank you for your advice and hospitality. Aiden, Matt and Martin, our collaboration unfortunately did not make it into this thesis, but I enjoyed working with you and learning from you. Thank you to the Explora team, Davide and Serena, for our VEGF collaboration. A big thank you to Aline, Shannon, Max and Fabio for the fun times at the meetings, especially in Galway. 
A special thank you goes to the team from Innocore. Our collaboration had its up and downs, but we had a strong finish and two nice publications resulted from it. My biggest thank you goes to Thanh, for managing our collaboration from Innocore's side and being so responsive and supporting. Thank you also to Rob, Ana, Lídia and Gert, as well as the entire Innocore team for your warm welcome while I was a visiting scientist in your labs. I learned a great deal while working with you that has already turned out to be valuable in my current job.

Thank you to the UCD team, Eoin, Stefano and Fergal for our fruitful collaboration on the beta-shells. Fergal, thank you for your work on the b-shells, your input and the trip to Palermo. Thank you for showing me the ins and out of 3D-printing in the "lab cellar" in Dublin.

Many people from our department have contributed to this work. Thank you Everaldo, Herre, Rene, Gert, Enrico, Massi and Sabrina for your expertise and advice. Everaldo, thank you for our chats over coffee every now and then and for organizing the Christmas dinner together. Thank you Tina, for our collaboration on the hydrogel project.

Big thank you to Mies, the all-round-technician. You contributed to all chapters of my thesis, be it with GPC, rheology, finding lab equipment, NMR, you name it. Thank you so much for your consistent quick help. When another professor introduces their head technician as "the Mies of our lab", that says enough. Thank you Marcel, for your help with HUVEC experiments and for your calming and positive nature. Thank you Joep, for your excellent assistance with everything regarding cell culture and for keeping the ML2 lab up and running. Thank you to Louis, for ordering materials for me, arranging shipments, and for keeping the biochemistry lab running and in order.

Barbara, thank you for your help regarding the administration and forms, for our occasional chats and your support, especially in the last months. I really enjoyed the "lab uitjes" and "karaoke borrels" with you!

Throughout the past years, many PhDs, students and post-docs joined and left the Pharmaceutics group. Thank you to all of you for the pleasant atmosphere and for any help in the lab, specifically Thijs, Erik OB, Jan-Jaap, Kristel, Mohammed, Anna, Andhyk, Haili, Martina, Jeffrey, Maarten, Genoveva, Cedric, Louann, Farshad, Levent, Danny, Lucia, Charis, Jerry, Yvonne, Mahsa, Boning, Irati, Vida, Neda, Aida, Alexandra, Cristina, Ada and Bárbara. Thank you Sjaak, for the fun SEM sessions, borrels and lab uitjes. Carl, thank you for input and expertise on everything related to the CSMA hydrogels. Maria, thanks for all the good times together and for your help in the lab at the start of my PhD. Thank you Gui, for the lunches, discussions and fun times together. Lies, thanks for the borrels and organizing the pub quiz together. Tamanna, thank you for our coffee sessions and for being such a nice colleague and friend. Marzieh, you were one of the first people I met from the Pharmaceutics group, and I want to thank you for being such a caring colleague. Yan, thank you for your help with GPC experiments and for our shared interest in cultures. Blessing, thank you for your help with the UPLC. Thank you to the 
Marko, Bo, Yong, Mathew, Erik D for the pleasant atmosphere and your support during the last months of my PhD. Amr, thank you for hosting me in Chicago and your friendship. Erik $H$, thanks for all the fun at borrels, your support and for just being a great colleague. Roderick, your help with the UPLC measurements was indispensable, thank you!

Thank you to my students, Sherilyn, Tayin, Sem and Manavi. I enjoyed supervising each and every one of you and you have all taught me a lot. Manavi, a big thank you for your work on the hydrogels!

I also want to thank Chris and Hans from the EM facility. Thank you for always being there to help and to answer questions, and reacting quickly when there were issues with the Phenom. Thanks to you, I was able to capture beautiful SEM images without any problems.

Thank you to the Schwendeman lab for welcoming me to your group. I had a fantastic stay in Ann Arbor. Thank you Steve, for your supervision, advice and feedback on my work. Thank you to the lab managers Karl and Rose, for your help with setting up experiments and any other questions that arose in the lab. Rose, thank you for our kayak trip. Maria, thanks for showing me around campus and for the lunches. Thank you to all PhDs, students and post-docs that I met during my stay for your help, pleasant tête-à-têtes and lunches.

Désirée and Johanna, I am extremely happy that you will be my paranymphs. Désirée, thank you for your support and advice, for bringing in a fresh perspective on things and for being so caring. Johanna, as a fellow German, we "clicked" right away. Thank you for your support, our laughs together and your quick help in desperate times.

I am extremely grateful for the support of my and Milan's loving family. Thank you for always being there for me and believing in me. Thank you to all my friends in Germany, Canada and the Netherlands for your support and encouragement in difficult times. Special thank you to the "Scandis" for the fun activities during the last years and for making me feel at home in the Netherlands. Thank you to Annelies, for our creative sessions, having coffees together and our friendship. Madlen, with you I could talk about so many things that only a fellow German living in the Netherlands could understand, thank you for that.

Last, and most importantly, thank you Milan, for your unconditional support and patience over the last few years. Thank you for our vacations throughout the last years, during which I could get my mind off of experiments and just relax. Here's to many more adventures together, from now on without any PhD worries.

Thank you to everybody!

Karina 

